\title{
Cervical vestibular evoked myogenic potentials
}

\author{
Citation for published version (APA):
}

Noij, K. S. (2020). Cervical vestibular evoked myogenic potentials: Toward optimizing clinical use. [Doctoral Thesis, Maastricht University]. GVO drukkers \& vormgevers B.V. https://doi.org/10.26481/dis.20200306kn

Document status and date:

Published: 01/01/2020

DOI:

10.26481/dis.20200306kn

Document Version:

Publisher's PDF, also known as Version of record

\section{Please check the document version of this publication:}

- A submitted manuscript is the version of the article upon submission and before peer-review. There can be important differences between the submitted version and the official published version of record. People interested in the research are advised to contact the author for the final version of the publication, or visit the DOI to the publisher's website.

- The final author version and the galley proof are versions of the publication after peer review.

- The final published version features the final layout of the paper including the volume, issue and page numbers.

Link to publication

\footnotetext{
General rights rights.

- You may freely distribute the URL identifying the publication in the public portal. please follow below link for the End User Agreement:

www.umlib.nl/taverne-license

Take down policy

If you believe that this document breaches copyright please contact us at:

repository@maastrichtuniversity.nl

providing details and we will investigate your claim.
}

Copyright and moral rights for the publications made accessible in the public portal are retained by the authors and/or other copyright owners and it is a condition of accessing publications that users recognise and abide by the legal requirements associated with these

- Users may download and print one copy of any publication from the public portal for the purpose of private study or research.

- You may not further distribute the material or use it for any profit-making activity or commercial gain

If the publication is distributed under the terms of Article $25 \mathrm{fa}$ of the Dutch Copyright Act, indicated by the "Taverne" license above, 


\section{Cervical vestibular evoked myogenic potentials}

\section{Toward optimizing clinical use}

Kimberley S. Noij 
This thesis was prepared at Massachusetts Eye and Ear, Harvard Medical School, Boston, USA and Maastricht University Medical Center, Maastricht, the Netherlands.

\section{Colofon}

(C) Copyright Kimberley S. Noij, Maastricht, 2020.

All rights reserved. No part of this book may be reproduced or transmitted in any form or by any means, without prior permission in writing by the author, or when appropriate, by the publishers of the publications.

ISBN: 978-94-6332-587-5

Cover/design: Garyfallia Pagonis

Layout: Tiny Wouters

Production: GVO drukkers \& vormgevers B.V.

The author gratefully acknowledges the financial support for publication of this thesis by: Atos Medical B.V., Beter Horen, ChipSoft, Cochlear Benelux N.V., Dos Medical BV/kno-winkel.nl, EmiD audiologische apparatuur, MED-EL, MediTop B.V., Mylan, PENTAX Medical, Phonak, Vestibulaire Vereniging. 


\title{
Cervical vestibular evoked myogenic potentials Toward optimizing clinical use
}

\author{
Proefschrift \\ ter verkrijging van de graad van doctor aan de Universiteit Maastricht, \\ op gezag van de Rector Magnificus, Prof. Dr. Rianne M. Letschert, \\ volgens het besluit van het College van Decanen, \\ in het openbaar te verdedigen \\ op vrijdag 6 maart 2020 om 12.00 uur
}

door

Kimberley S. Noij

geboren op 20 december 1989

te Badhoevedorp 
Doctoral advisors - Promotoren

Prof. dr. H. Kingma

Universiteit Maastricht

Prof. S.D. Rauch, MD

Massachusetts Eye and Ear, Harvard Medical School

Co-doctoral advisor - Copromotor

Dr. R. van de Berg

Universiteit Maastricht

\section{Dissertation committee - Beoordelingscommissie}

Prof. dr. B. Kremer

Prof. J. P. Carey, MD

Prof. dr. W.H. Mess

Prof. dr. V. van Rompaey

Prof. dr. Y. Temel
Universiteit Maastricht (Chair - voorzitter)

Johns Hopkins University School of Medicine

Universiteit Maastricht

Universiteit Antwerpen

Universiteit Maastricht 
"After climbing a great hill, one only finds that there are many more hills to climb."

- Nelson Mandela 



\section{Contents}

Chapter 1. Introduction 9

1.1 General introduction $\quad 11$

$\begin{array}{ll}1.2 \text { Aims and outline } & 29\end{array}$

Chapter 2. Toward optimizing cervical vestibular evoked myogenic potentials $\quad 35$

2.1 Calculating VEMP inhibition depth with a generic template $\quad 37$

2.2 Normalization reduces the need for strong muscle contraction $\quad 57$

Chapter 3. Clinical use of cVEMP in patients with superior semicircular 75

canal dehiscence syndrome

3.1 Combining air-bone gap and cVEMP thresholds to improve diagnosis of superior canal dehiscence syndrome

3.2 Audiometric and cVEMP thresholds show little correlation with symptoms in superior semicircular canal dehiscence syndrome

3.3 $2000 \mathrm{~Hz}$ tone bursts improve the detection of superior semicircular canal dehiscence syndrome

Chapter 4. Clinical use of cVEMP in Menière's disease patients

4.1 Cervical vestibular evoked myogenic potentials in Menière's disease: A comparison of response metrics

4.2 Predicting development of bilateral Menière's disease based on cVEMP threshold and tuning

Chapter 5. Discussion

Chapter 6. Summary

Chapter 7. Dutch Summary - Nederlandse Samenvatting

Acknowledgements - Dankwoord

Bibliography

Curriculum vitae 



\section{Chapter 1}

Introduction 



\section{Chapter 1.1}

General introduction 



\section{General introduction}

\section{The vestibular system}

The vestibular system is one of the three major pillars that play a role in spatial orientation and balance. The brain integrates visual, proprioceptive and vestibular information to maintain balance (1). The vestibular apparatus is located in the inner ear and, together with the cochlea (the hearing organ), forms the labyrinth (Figure 1.1.1 and 1.1.2). Each labyrinth contains five vestibular components: three semicircular canals and two otolith organs (the saccule and utricle). The superior, horizontal and posterior semicircular canals detect angular acceleration in their respective planes. The saccule and utricle mainly detect linear acceleration and head tilt in the vertical and horizontal plane respectively (Figure 1.1.2). These otolith organs are also able to detect rotation using centrifugal forces. The sensory organs of the semicircular canals, the cupulae, are located in the ampulla of each canal and consist of hair cells that are stimulated by movement (Figure 1.1.2). The hair cells of the saccule and utricule are located in the maculae. On top of the macular hair cells lies the otolithic membrane, containing otoconia. Otoconia are composed of calcium carbonate. Movement displaces their mass, stimulating the hair cells (Figure 1.1.2). The superior and horizontal semicircular canals, utricle and part of the saccule are innervated by the superior vestibular nerve, while the posterior semicircular canal and part of the saccule are innervated by the inferior vestibular nerve (Figure 1.1.2) (2). All five vestibular components stimulate their nerves, which travel to the vestibular nuclei, located in the brainstem. From the brainstem, projections to the eyes and muscles aid in visually focusing on an object and maintaining balance, also referred to as vestibular reflexes (3). Projections to central vestibular areas exist as well, including the cortex, thalamus, hippocampus and cerebellum. These structures play a role in higher vestibular function by integrating multisensory input (4).

\section{Acoustic stimulation}

When the ear is stimulated by sound, sound pressure waves travel through the external ear canal to the tympanic membrane (Figure 1.1.1). In humans, vibration of the tympanic membrane leads to movement of the ossicles (malleus, incus and stapes), which creates a pressure wave in the labyrinth. The pressure difference created across the basilar membrane in the cochlea stimulates the cochlear hair cells, located on top of the basilar membrane, and contributes to hearing (Figure 1.1.2). Sound can also directly cause skull vibration, stimulating the cochlear hair cells and bypassing the outer and middle ear.

Fish, reptiles and amphibians do not have a cochlea and use their otolith organs for hearing and balance $(5,6)$. The ability to stimulate the vestibular sense organs with 
sound is maintained in mammals and may be a remnant with no particular function in humans, although a saccular role in sensing musical rhythm has been suggested $(5,7-$ 11). Roughly, there are two types of vestibular afferents: regular and irregular firing afferents. From single motor unit recordings in guinea pigs and cats it was determined that only a subset of the irregular firing otolith afferents respond to sound and that the large majority of regular firing and semicircular canal afferents cannot be activated with non-traumatic sounds $(9,10,12-14)$.

The saccule specifically lends itself to air-conducted sound stimulation (ACS), potentially due to its close proximity to the stapes footplate, although some utricular afferents respond to ACS as well $(8-10,15-17)$. The same pathway from the saccule to the neck muscles used to maintain the head upright with movement can also be activated when the saccule is acoustically stimulated. This vestibulocollic pathway extends from the saccule to vestibular afferents reaching the vestibular nuclei in the brainstem. These nuclei have projections to descending spinal tracts and the accessory nucleus, which then further project to neck muscle motoneurons. A neck muscle response, caused by acoustic stimulation of the saccule cannot visually be observed and may not have a specific function in humans (12).

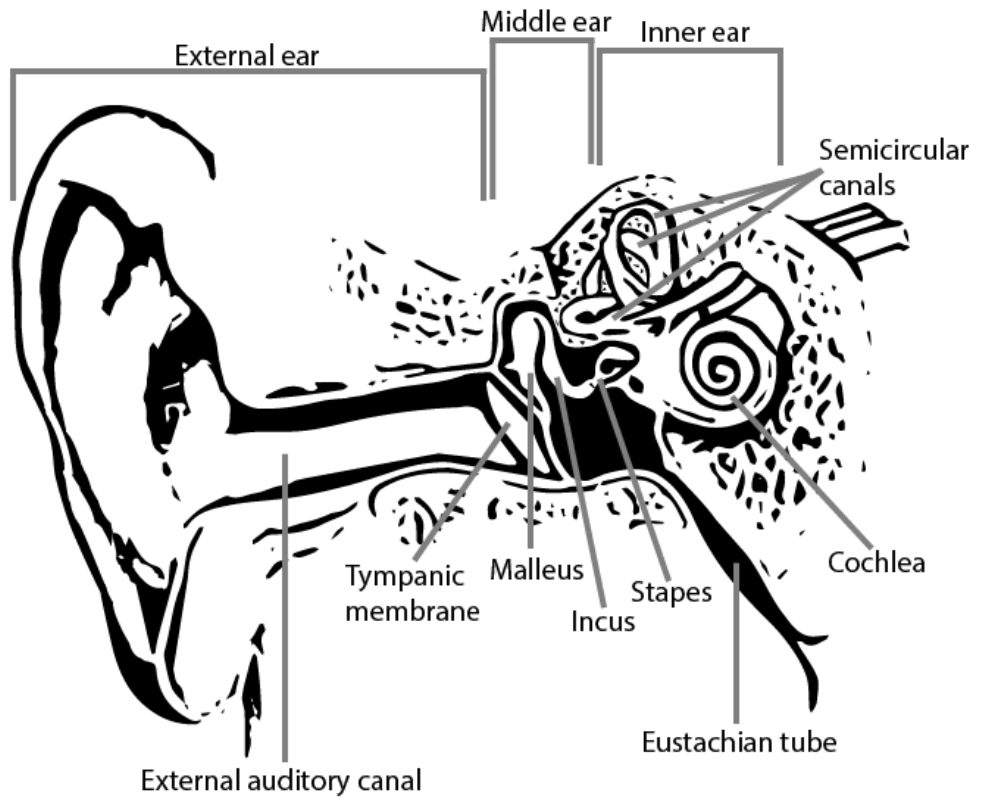

Figure 1.1.1 Anatomy of the external, middle and inner ear. 


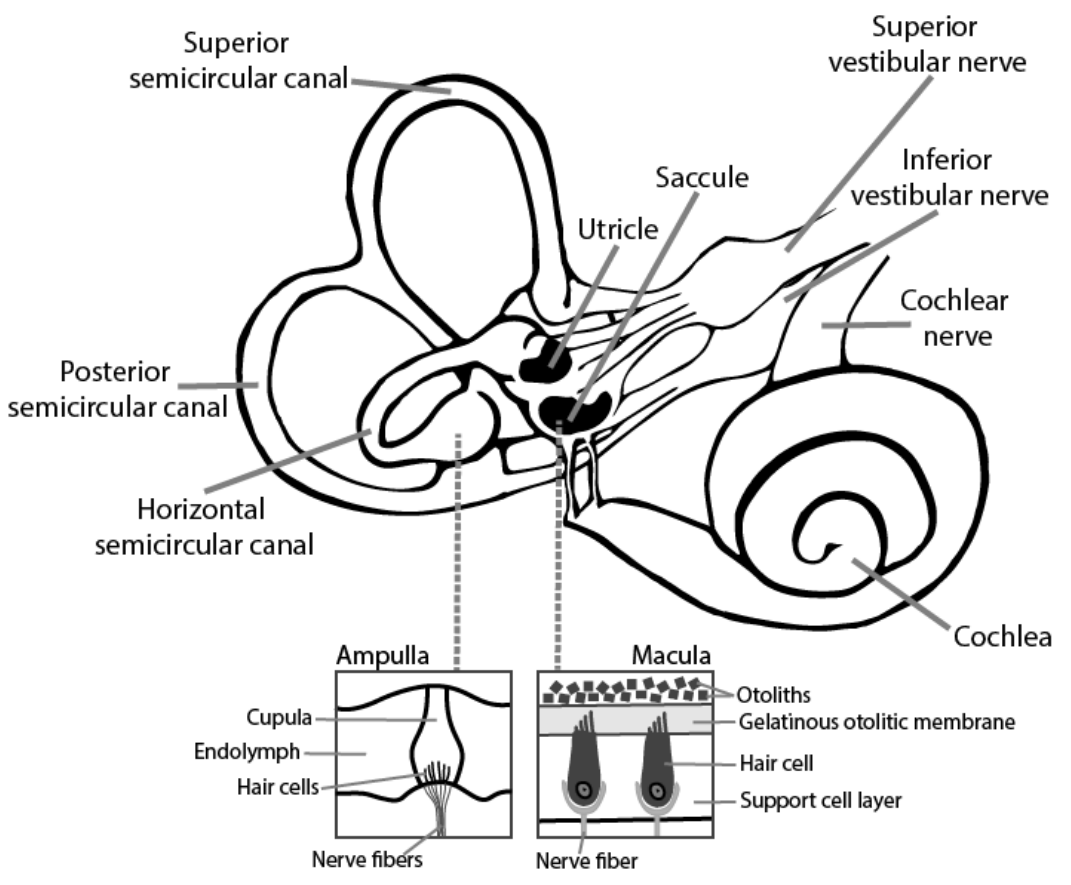

Figure 1.1.2 Overview of the inner ear. The inner ear is comprised of the labyrinth, which includes the cochlea and vestibular apparatus. The vestibular apparatus consists of the three semicircular canals and two otolith organs (saccule and utricle). Detailed schematics of the semicircular canal sense organs (ampulla) and otolith organs (macula) are provided.

\section{Vestibular testing}

Currently, all five vestibular sense organs can be tested separately. The video head impulse test (VHIT) measures responses for each of the three semicircular canals to head rotations in their respective planes. During this test a fast head movement elicits eye movements in the opposite direction. Both head and eye movement are recorded with a video camera. These movements are measured and plotted in the same graph, allowing for their comparison (18). Caloric and rotatory chair testing can be used to test mainly the horizontal canals (19). The caloric test measures each ear separately. During rotary chair testing, both ears are rotating, so in pathologic cases the test cannot ascertain which is the affected side.

The utricles can be assessed using the subjective visual vertical and ocular counter roll tests. The saccules partially contribute to these tests as well, so these tests are unable to completely isolate the otolith organs nor can the left and right sides be evaluated separately (20). The development of vestibular evoked myogenic potential testing enabled the ability to assess the otolith organs separately, with the ability to isolate the 
left and the right side. The cervical vestibular evoked myogenic potential (cVEMP) relies on the vestibulocollic reflex and assesses saccular and inferior vestibular nerve function through ipsilateral inhibition of the neck muscles. The ocular vestibular evoked myogenic potential (oVEMP) uses vestibulo-ocular projections, allowing for the assessment of utricular and superior vestibular nerve function through contralateral excitation of the eye muscles (12).

Before the development of the vestibular evoked myogenic potential (VEMP), the saccule and utricle could not be evaluated individually and to this day, the VEMP is the only test that does.

\section{The cervical vestibular evoked myogenic potential}

In the 1960's, Bickford and others investigated cortical responses to auditory stimulation and discovered a vestibular reflex during their studies. While recording electroencephalographic (EEG) responses to loud clicks, they found a response at the inion that could be elicited in patients with complete sensorineural hearing loss and normal vestibular function, but not in patients with loss of both hearing and vestibular function, indicating a vestibular origin (21). Studies in guinea pigs and cats indicated that this vestibular evoked myogenic response probably originated from the saccule $(8,9)$. This was confirmed in later animal studies, which also indicated that the nerve fibers involved in this response run through the inferior vestibular nerve $(8-10,14-$ $17,22)$.

In the 1990's, Colebatch and Halmagyi recorded vestibular evoked potentials from the sternocleidomastoid muscle (SCM) instead of the inion, allowing them to study the laterality of the reflex (23). In this study, a patient with Menière's disease (MD) was tested and a response could only be evoked during ipsilateral SCM contraction. The response disappeared after vestibular neurectomy (23). This ipsilateral SCM response was further investigated and could be separated into an early vestibular and a late cochlear component. The early component being the cervical vestibular evoked myogenic potential (cVEMP) (24). Based on single motor unit animal recordings it was determined that the CVEMP is inhibitory in nature, predominantly of saccular origin, and provides a measure of integrity of the saccule and inferior vestibular nerve $(12,25)$. This work indicated that the CVEMP had great potential to be a quick and noninvasive manner to test saccular and inferior vestibular nerve function in patients. The CVEMP was further developed and is now used in many clinics.

In the clinical setting, the cVEMP is obtained by acoustically or mechanically stimulating the saccule while the patient voluntarily contracts their ipsilateral SCM. The ipsilateral inhibition of the SCM, produced by the vestibulocollic reflex is measured with surface electromyography (EMG) electrodes on the tonically contracted SCM (Figure 1.1.3) (24). A typical CVEMP consists of two peaks, a first positive peak (P1) and a first negative peak (N1) occurring about 13 and 23 ms after sound onset for stimulation with clicks 
(Figure 1.1.4) (24). With tone bursts, the latency of the peaks depend on the rise time of the stimulus (26).

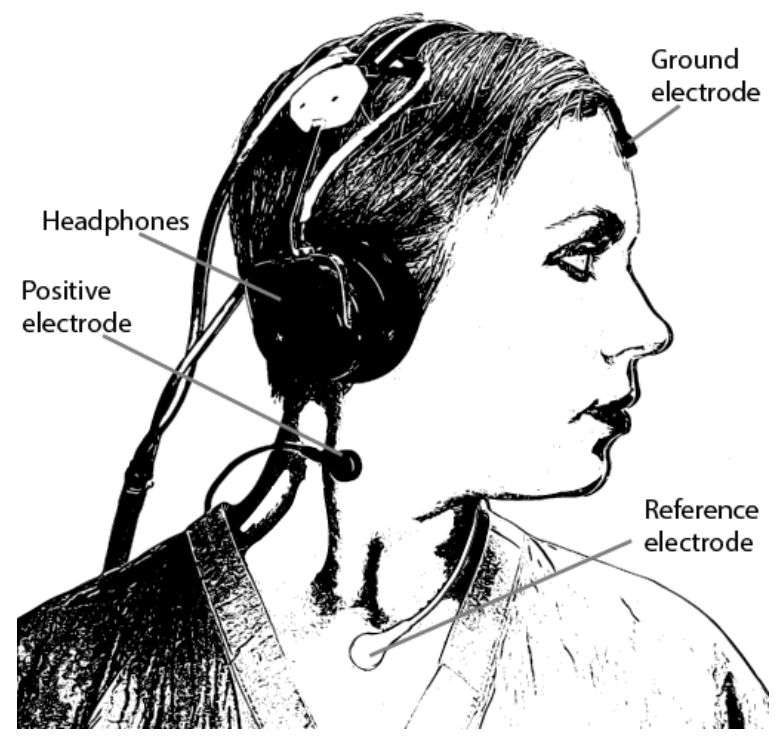

Figure 1.1.3 Example of cVEMP set-up during acoustic stimulation. The subject is wearing headphones through which an auditory stimulus is provided. A positive electrode is placed on the middle belly of each sternocleidomastoid muscle (only the right side is visible in this figure), a reference electrode is placed on the manubrium sterni and a ground electrode on the midline forehead.

\section{cVEMP metrics}

To assess saccular function with the cVEMP, multiple metrics can be used. The ideal cVEMP metric would solely reflect saccular and inferior vestibular nerve function. However, the cVEMP is affected by other factors and it is well-known that the amplitude of a cVEMP response varies greatly from person to person, even in a healthy population (26). This variability confounds the ability of the CVEMP to detect changes in saccular function. Previous studies aimed to decrease this variability. For example, the size of the response is much influenced by muscle contraction and the use of normalization methods significantly reduced the variability created by this muscle contraction effect (26). Another study attempted to remove variability from factors other than saccular function by estimating the fractional inhibition of SCM motoneurons, produced by saccular activation (27). These are just two possible means to decrease cVEMP variability in order to evaluate saccular function and it is not evident which metric (if any) is superior or, more clinically relevant, which metrics 
assists in diagnosis, follow-up and perhaps even prediction of disease. It is important to appreciate the various possibilities of assessing the CVEMP, and thereby saccular function.

First, the size of the response can be obtained by calculating the peak-to-peak amplitude (VEMPpp) of the cVEMP. VEMPpp is the difference in voltage between P1 and N1 (Figure 1.1.4). The VEMPpp is influenced by muscle contraction strength, with stronger contractions eliciting larger VEMPpp. Institutions use various ways to decrease the variability that originates from differences in muscle contraction, including: using a single target contraction level (28-30), a minimum target contraction level (26,31), a target range within which subjects have to maintain contraction (32), using a pressure cuff which subjects have to press their head against to obtain a certain pressure (33-35) and asking subjects to maintain a certain head position (e.g. turn head 45 degrees) $(36,37)$. These methods, however, do not prevent muscle contraction variability between and within subjects, and it is often challenging for patients to maintain a certain muscle contraction target. To correct for the variability in muscle contraction both between and within subjects, various normalization techniques have been developed using computational methods to correct for muscle contraction in an automatic fashion (38). Normalization can be used if the VEMPpp grows linearly with the muscle contraction level and many studies reported that this is the case, but that VEMPpp can saturate at high muscle contraction levels $(24,28,29,36,37,39-42)$. This saturation can affect the reliability of normalization. A variety of target levels have been proposed and recommended, indicating that it is unclear within what range normalization works well and if this is the same across ages and sound levels (28$30,37,43,44)$.

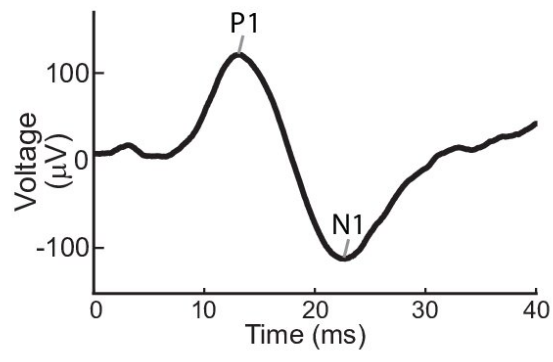

Figure 1.1.4 Example cVEMP waveform to a click with P1 and N1 occurring at approximately 13 and $23 \mathrm{~ms}$ respectively.

A different metric that was developed to provide normalization is the VEMP inhibition depth (VEMPid). The VEMPid estimates the fractional inhibition of SCM motoneuron response produced by activation of the saccule. This metric seems to be more sensitive than normalized peak-to-peak amplitudes (VEMPn) at low response levels and might 
therefore be more precise in evaluating saccular function than VEMPn. The VEMPid is computed using a template correlation method. This method resembles a matched filter for the detection of a response (the CVEMP) in noise. That is, the CVEMP response of the tested subject is compared to a template that serves as an example of what their cVEMP "should" look like. To calculate VEMPid, a template is needed and a cVEMP response obtained at a high sound level was originally used as the template in a group of healthy subjects tested to develop the VEMPid (27). The use of a subject's own cVEMP response as the template becomes problematic when patients with suspected vestibular pathology are tested, because they might not have a robust CVEMP response. A possible solution to this problem would be the use of a generic template.

The calculation of VEMPid with a generic template needs to accommodate the latency of the patient's CVEMP response, a metric not commonly used to evaluate peripheral vestibular pathology. Latency is expressed as the time (in ms) it takes for P1 and N1 to occur, after stimulation is started. The cVEMP latency does not seem to be affected by peripheral vestibular pathology, while retro-labyrinthine pathologies along the vestibulocollic pathway, such as demyelinating disease (e.g. multiple sclerosis), cerebrovascular disease, neurodegenerative disease and vestibular schwannomas are more likely to show prolonged latencies (45-47). The VEMPid can only be used if the template is matched with the subject's response. If their latencies differ, then the VEMPid cannot reliably be computed. Reported "normal" cVEMP latencies vary and are dependent on factors such as stimulus (click versus tone burst) and frequency (latency seems to be dependent on the rise-time of the tone burst stimulus) $(26,48,49)$. To calculate VEMPid with a generic template, a correction must be made for this variability in latency.

The next metric, not containing information about response size or latency, is the cVEMP threshold. The threshold is defined as the lowest sound level that can elicit a cVEMP response. In previous reports, threshold seemed to be valuable in 1) distinguishing ears in patients with unilateral Menière's Disease (affected vs. unaffected vs. normal) (50), 2) distinguishing patients with migraine associated vertigo and Menière's disease, as well as tracking disease progression in these groups (51) and 3 ) evaluating patients with semicircular canal dehiscence syndrome (SCD) (52-54).

Another commonly used metric to evaluate patients is the inter-aural asymmetry ratio (IAR). The IAR reflects the difference in amplitude between both ears. There are multiple ways to calculate the IAR (55-63). As with latency, there is large variability in IAR in normal subjects (60-62). Another limitation of the IAR is that in patients with unilateral Menière's disease (MD) the unaffected ears have altered cVEMP thresholds and amplitudes compared to healthy controls and about a quarter to one third of both MD and SCD patients have, or eventually develop, bilateral disease (64-71). Because especially in MD patients, the IAR is such a widely used metric, it would be valuable to determine the sensitivity and specificity to distinguish affected MD from healthy control ears and compare these to other CVEMP metrics. 
The last cVEMP metric evaluated in this thesis is the frequency tuning ratio. An example is the $500 / 1000 \mathrm{~Hz}$ cVEMP threshold ratio, which is calculated by dividing the $500 \mathrm{~Hz}$ cVEMP threshold by the $1000 \mathrm{~Hz}$ cVEMP threshold of the same subject at the same side. Multiple studies evaluating MD patients found a frequency tuning shift to a higher frequency in these patients compared to healthy controls $(50,55,58,72,73)$. This means that the largest cVEMP response that can be elicited, shifts from a lower to a higher frequency. This tuning shift is thought to originate from increased stiffness of the saccular membrane in MD patients due to endolymphatic hydrops (50).

Few studies investigated the sensitivity and specificity of some of the cVEMP metrics in differentiating patient groups from healthy controls (74). Most studies were small and methodological differences between them limits their comparison. It would be valuable to know how sensitive and specific the different cVEMP metrics are in differentiating patient groups from healthy controls in a larger group of patients. Furthermore, it is important to know which metric does this best. Last, it has been hypothesized that the cVEMP may be valuable in predicting which unilateral MD patients will develop bilateral disease (75). To date, no study has aimed to test this hypothesis.

\section{Clinical use}

As previously indicated, the cVEMP can be used to clinically assess patients with vestibular pathology. As a diagnostic tool, the cVEMP seems most useful in patients with superior semicircular canal dehiscence syndrome (SCD). Although the CVEMP seems less valuable in diagnosing other vestibular pathologies, it may be used for the follow-up of these patients (74). The two vestibular pathologies that this thesis will focus on are SCD and Menière's disease.

\section{Superior semicircular canal dehiscence syndrome}

Superior semicircular canal dehiscence is a defect in the bony covering of the superior semicircular canal. This dehiscence can result in an array of auditory and vestibular symptoms, including hearing loss, autophony, tinnitus, hyperacusis, imbalance and sound, pressure and exercise induced vertigo. The combination of the anatomical defect and symptoms is referred to as semicircular canal dehiscence syndrome (Figure 1.1.5) (76). It is uncertain exactly how prevalent this syndrome is. A cadaver study reported the presence of superior semicircular canal dehiscence in $0.5 \%$ of ears, while a study using CT scans reported a prevalence of $4 \%(77,78)$. SCD has been reported to occur bilaterally in about a quarter of patients (64). 

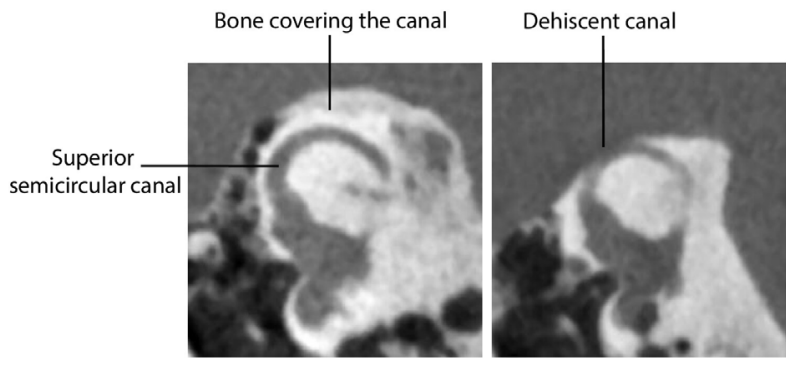

Figure 1.1.5 Example of a normal superior semicircular canal on the left with bone covering the canal and a dehiscent superior semicircular canal on the right with an absence of bone covering part of the canal (Pöschl view: plane parallel to superior semicircular canal).

The symptoms that SCD patients suffer from are thought to occur because of a "third window" mechanism that exists in the presence of a dehiscence. In a healthy ear, sound entering the ear leads to movement of the stapes footplate, creating a pressure wave in the labyrinth and an equal outward motion of the round window (two window mechanism). In the presence of a dehiscence, the energy created by the stapes footplate motion shunts towards the third window causing a decrease in pressure difference across the basilar membrane and an increase in energy transmission to the vestibular sense organs (Figure 1.1.6) $(79,80)$. In theory, more energy transmission at the level of the saccule would result in larger cVEMP responses, lower CVEMP thresholds and a more symptomatic patient. Clinically, the presence and severity of symptoms that occur in SCD patients vary greatly and the relationship between CVEMP outcomes and symptomatology will be discussed in this thesis.

A dehiscence of the superior semicircular canal can be detected with high resolution computed tomography (CT) imaging of the temporal bone (76). Although CT scans were considered the gold standard in diagnosing SCD, they are relatively expensive and expose patients to radiation. The symptoms that can occur in SCD are not unlike the symptoms that can be present in other otologic diseases. It may therefore not be desirable to scan all patients who present with these symptoms. Furthermore, due to volume averaging, CT scans tend to overestimate the dehiscence leading to false positive findings $(81,82)$. In theory, the accuracy of the CT scan would improve by decreasing the slice thickness with which these scans are obtained. The issue arising with the use of a smaller slice thickness is that this would increase the risk for motion artefacts as well as the amount of radiation exposure. 

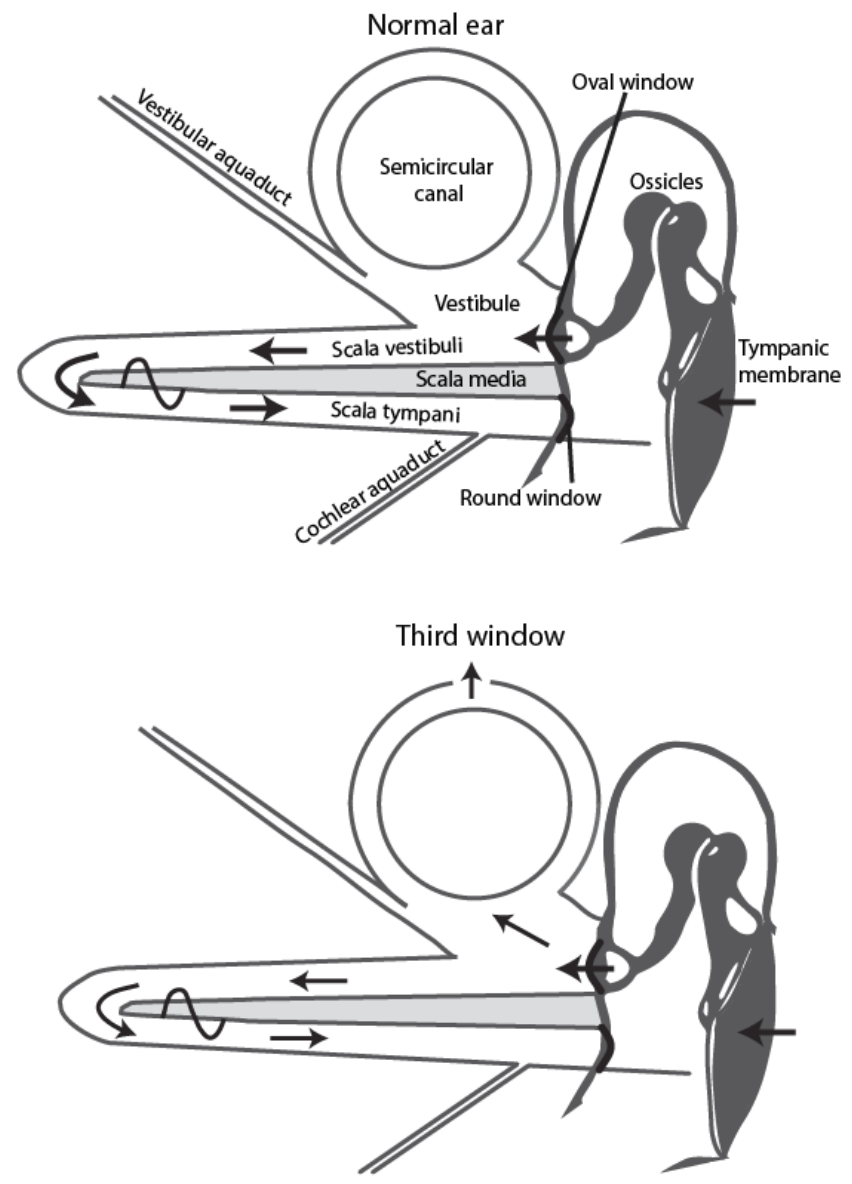

Figure 1.1.6 Overview of the healthy two (top) and third (bottom) window mechanism during air-conducted stimulation of the ear. The vestibule (labeled in the top panel) contains the saccule and utricle. This figure was based on a figure published by Ho et al. 2016 (Figure 1) (80).

VEMP testing seems to be a useful addition to the CT scan in diagnosing SCD patients and currently, the diagnosis is often based on a combination of clinical findings, audiometric testing, VEMP testing and imaging. The use of all these different tests is costly and time consuming. It would be desirable to have a screening test for SCD, which would distinguish it from other otologic diseases. The cVEMP has the potential to become such a screening test, because SCD patients have significantly lower thresholds and higher amplitudes compared to non-SCD patients $(83,84)$. However, there is overlap in cVEMP outcomes with the normal population and the reported sensitivity, 
specificity and positive predictive value have been suboptimal for its use as a screening tool $(85,86)$.

As described above, a variety of cVEMP metrics as well as different frequencies can be used to evaluate SCD patients. Studies often assess only few metrics and frequencies and do not always provide sensitivities and specificities. Because of methodological differences between studies, it is difficult to compare outcomes between them. For optimal clinical use of the CVEMP, it is key to verify which metric and frequency best differentiate dehiscent ears from healthy controls and how accurate they are.

\section{Menière's disease}

Patients with Menière's disease (MD) suffer from a combination of fluctuating sensorineural hearing loss, tinnitus or aural fullness, and vertigo attacks (87). The reported prevalence of Menière's disease ranges from 0.001 to $0.22 \%(67,88-91)$. Endolymphatic hydrops in the inner ear resulting in degeneration of the saccule appears to be the underlying pathology and cause of the vestibular symptoms (92). Although attempts have been made to visualize the presence of endolymphatic hydrops in vivo, its presence does not define $M D$, i.e. not everyone with endolymphatic hydrops has MD (93).

Since the CVEMP is the only available test to evaluate saccular function, it has been used to assess patients with MD. MD patients generally have smaller amplitudes, higher thresholds and altered frequency tuning compared to healthy controls $(50,55,94)$. Interestingly, the same applies to affected and unaffected ears of MD patients, with the affected ears being more "abnormal" $(50,75)$. Since MD occurs bilaterally in approximately a quarter to a third of patients, this has raised the question if the CVEMP outcomes of these unaffected ears could provide information about which patients develop bilateral disease $(50,65-71,75)$.

Previous cVEMP studies evaluating MD patients often have assessed only one or two metrics, differed in methodology and rarely provided sensitivities and specificities. This prevents a reliable comparison across studies. 


\section{References}

1. Angelaki DE, Cullen KE. Vestibular system: the many facets of a multimodal sense. Annu Rev Neurosci 2008;31:125-50.

2. Baloh RW, Honrubia V. Clinical Neurophysiology of the Vestibular System. Edition 2. 1990. Chapter 1 and 2.

3. Baloh RW, Honrubia V. Clinical Neurophysiology of the Vestibular System. Edition 2. 1990. Chapter 3.

4. Brandt T, Strupp M, Dieterich M. Towards a concept of disorders of "higher vestibular function". Front Integr Neurosci 2014;8:47.

5. Todd N. Evidence for a behavioral significance of saccular acoustic sensitivity in humans. $J$ Acoust Soc Am 2001;110(1):380-90.

6. Fay RR, Popper AN. Evolution of hearing in vertebrates: the inner ears and processing. Hear Res 2000; 149(1-2):1-10.

7. Young ED, Fernandez C, Goldberg JM. Responses of squirrel monkey vestibular neurons to audiofrequency sound and head vibration. Acta Otolaryngol 1977;84(5-6):352-60.

8. Didier A, Cazals Y. Acoustic responses recorded from the saccular bundle on the eighth nerve of the guinea pig. Hear Res 1989;37(2):123-7.

9. McCue MP, Guinan JJ Jr. Acoustically responsive fibers in the vestibular nerve of the cat. J Neurosci 1994;14(10):6058-70.

10. Murofushi T, Curthoys IS, Topple AN, et al. Responses of guinea pig primary vestibular neurons to clicks. Exp Brain Res 1995; 103(1):174-8.

11. Todd NP, Cody FW. Vestibular responses to loud dance music: a physiological basis of the "rock and roll threshold"? J Acoust Soc Am 2000;107(1):496-500.

12. Curthoys IS. A critical review of the neurophysiological evidence underlying clinical vestibular testing using sound, vibration and galvanic stimuli. Clin Neurophysiol 2010;121(2):132-44.

13. McCue MP, Guinan JJ Jr. Spontaneous activity and frequency selectivity of acoustically responsive vestibular afferents in the cat. J Neurophysiol 1995;74(4):1563-72.

14. McCue MP, Guinan JJ Jr. Sound-evoked activity in primary afferent neurons of a mammalian vestibular system. Am J Otol 1997;18(3):355-60.

15. Murofushi T, Curthoys IS. Physiological and anatomical study of click-sensitive primary vestibular afferents in the guinea pig. Acta Otolaryngol 1997;117(1):66-72.

16. Zhu H, Tang X, Wei W, et al. Input-output functions of vestibular afferent responses to air-conducted clicks in rats. J Assoc Res Otolaryngol 2014;15(1):73-86.

17. Curthoys IS, Vulovic V, Burgess AM, et al. The response of guinea pig primary utricular and saccular irregular neurons to bone-conducted vibration (BCV) and air-conducted sound (ACS). Hear Res 2016;331:131-43.

18. Halmagyi GM, Chen L, MacDougall HG, et al. The Video Head Impulse Test. Front Neurol 2017;8:258.

19. Fife TD, Tusa RJ, Furman JM, et al. Assessment: vestibular testing techniques in adults and children: report of the Therapeutics and Technology Assessment Subcommittee of the American Academy of Neurology. Neurology 2000;55(10):1431-41.

20. Rosengren SM, Colebatch JG. The contributions of vestibular evoked myogenic potentials and acoustic vestibular stimulation to our understanding of the vestibular system. Front Neurol 2018;9:481.

21. Bickford RG, Jacobson JL, Cody DT. Nature of average evoked potentials to sound and other stimuli in man. Ann N Y Acad Sci 1964;112:204-23.

22. Tsubota $\mathrm{M}$, Shojaku $\mathrm{H}$, Hori $\mathrm{E}$, et al. Effects of vestibular nerve section on sound-evoked myogenic potentials in the sternocleidomastoid muscle of monkeys. Clin Neurophysiol 2007;118(7):1488-93.

23. Colebatch JG, Halmagyi GM. Vestibular evoked potentials in human neck muscles before and after unilateral vestibular deafferentation. Neurology 1992;42(8):1635-6.

24. Colebatch JG, Halmagyi GM, Skuse NF. Myogenic potentials generated by a click-evoked vestibulocollic reflex. J Neurol Neurosurg Psychiatry 1994;57(2):190-7.

25. Colebatch JG, Rothwell JC. Motor unit excitability changes mediating vestibulocollic reflexes in the sternocleidomastoid muscle. Clin Neurophysiol 2004;115(11):2567-73. 
26. van Tilburg MJ, Herrmann BS, Guinan JJ Jr, et al. Normalization reduces intersubject variability in cervical vestibular evoked myogenic potentials. Otol Neurotol 2014;35(8):e222-7.

27. Prakash SR, Herrmann BS, Milojcic R, et al. Evaluating inhibition of motoneuron firing from electromyogram data to assess vestibular output using vestibular evoked myogenic potentials. Ear Hear 2015;36(5):591-604.

28. Akin FW, Murnane OD, Panus PC, et al. The influence of voluntary tonic EMG level on the vestibularevoked myogenic potential. J Rehabil Res Dev 2004;41(3B):473-80.

29. McCaslin DL, Fowler A, Jacobson GP. Amplitude normalization reduces cervical vestibular evoked myogenic potential (cVEMP) amplitude asymmetries in normal subjects: proof of concept. J Am Acad Audiol 2014;25(3):268-77.

30. Davenport MJ. The effect of sternocleidomastoid muscle activation pattern and feedback condition on the vestibular evoked myogenic potential. Dissertation. 2010, East Tennessee State University. p. 164.

31. McCaslin DL, Jacobson GP, Hatton K, et al. The effects of amplitude normalization and EMG targets on cVEMP interaural amplitude asymmetry. Ear Hear 2013;34(4):482-90.

32. Basta D, Todt I, Ernst A. Characterization of age-related changes in vestibular evoked myogenic potentials. J Vestib Res 2007;17(2-3):93-8.

33. Lee KJ, Kim MS, Son EJ, et al. The usefulness of rectified VEMP. Clin Exp Otorhinolaryngol 2008;1(3): 143-7.

34. Tourtillott BM, Ferraro JA, Bani-Ahmed A, et al. Age-related changes in vestibular evoked myogenic potentials using a modified blood pressure manometer feedback method. Am J Audiol 2010;19(2): 100-8.

35. Maes L, Vinck BM, De Vel E, et al. The vestibular evoked myogenic potential: a test-retest reliability study. Clin Neurophysiol 2009;120(3):594-600.

36. Bogle JM, Zapala DA, Criter R, et al. The effect of muscle contraction level on the cervical vestibular evoked myogenic potential (cVEMP): usefulness of amplitude normalization. I Am Acad Audiol 2013;24(2):77-88.

37. Rosengren SM. Effects of muscle contraction on cervical vestibular evoked myogenic potentials in normal subjects. Clin Neurophysiol 2015;126(11):2198-206.

38. van Tilburg MJ, Herrmann BS, Rauch SD, et al. Normalizing cVEMPs: Which method is the most effective? Ear Hear 2018.

39. Akin FW, Murnane OD. Vestibular evoked myogenic potentials: preliminary report. J Am Acad Audiol 2001;12(9):445-52; quiz 491.

40. Isaacson B, Murphy E, Cohen H. Does the method of sternocleidomastoid muscle activation affect the vestibular evoked myogenic potential response? J Vestib Res 2006;16(4-5):187-91.

41. Lim CL, Clouston P, Sheean G, et al. The influence of voluntary EMG activity and click intensity on the vestibular click evoked myogenic potential. Muscle Nerve 1995;18(10):1210-3.

42. Ochi K, Ohashi T, Nishino H. Variance of vestibular-evoked myogenic potentials. Laryngoscope 2001; 111(3):522-7.

43. Rosengren SM, Welgampola MS, Colebatch JG. Vestibular evoked myogenic potentials: past, present and future. Clin Neurophysiol 2010;121(5):636-51.

44. Papathanasiou ES, Murofushi T, Akin FW, et al. International guidelines for the clinical application of cervical vestibular evoked myogenic potentials: an expert consensus report. Clin Neurophysiol 2014;125(4):658-66.

45. Roditi RE, Eppsteiner RW, Sauter TB, et al. Cervical vestibular evoked myogenic potentials (cVEMPs) in patients with superior canal dehiscence syndrome (SCDS). Otolaryngol Head Neck Surg 2009;141(1): 24-8.

46. Murofushi T, Shimizu K, Takegoshi H, et al. Diagnostic value of prolonged latencies in the vestibular evoked myogenic potential. Arch Otolaryngol Head Neck Surg 2001;127(9):1069-72.

47. Venhovens J, Meulstee J, Verhagen WIM. Vestibular evoked myogenic potentials (VEMPs) in central neurological disorders. Clin Neurophysiol 2016;127(1):40-9.

48. Meyer N, Vinck B, Heinze B. cVEMPs: a systematic review and meta-analysis. Int J Audiol 2015;54(3): 143-51. 
49. Rahne T, Weiser C, Plontke S. Neurofeedback-controlled comparison of the head elevation versus head rotation and head-hand methods in eliciting cervical vestibular evoked myogenic potentials. Audiol Neurootol 2014;19(5):327-35.

50. Rauch SD, Zhou G, Kujawa SG, et al. Vestibular evoked myogenic potentials show altered tuning in patients with Meniere's disease. Otol Neurotol 2004;25(3):333-8.

51. van Tilburg MJ, Herrmann BS, Guinan JJ Jr, et al. Serial cVEMP Testing is Sensitive to Disease Progression in Meniere Patients. Otol Neurotol 2016;37(10):1614-9.

52. Niesten ME, McKenna MJ, Herrmann BS, et al. Utility of cVEMPs in bilateral superior canal dehiscence syndrome. Laryngoscope 2013;123(1):226-32.

53. Welgampola MS, Myrie OA, Minor LB, et al. Vestibular-evoked myogenic potential thresholds normalize on plugging superior canal dehiscence. Neurology 2008;70(6):464-72.

54. Hunter JB, Patel NS, O'Connell BP, et al. Cervical and ocular VEMP testing in diagnosing superior semicircular canal dehiscence. Otolaryngol Head Neck Surg 2017;156(5):917-923.

55. Salviz M, Yuce T, Acar H, et al. Diagnostic value of vestibular-evoked myogenic potentials in Meniere's disease and vestibular migraine. J Vestib Res 2016;25(5-6):261-6.

56. Taylor RL, Wijewardene AA, Gibson WP, et al. The vestibular evoked-potential profile of Meniere's disease. Clin Neurophysiol 2011; 122(6):1256-63.

57. Kim-Lee Y, Ahn JH, Kim YK, et al. Tone burst vestibular evoked myogenic potentials: diagnostic criteria in patients with Meniere's disease. Acta Otolaryngol 2009;129(9):924-8.

58. Maxwell R, Jerin C, Gurkov R. Utilisation of multi-frequency VEMPs improves diagnostic accuracy for Meniere's disease. Eur Arch Otorhinolaryngol 2017;274(1):85-93.

59. Egami N, Ushio M, Yamasoba T, et al. The diagnostic value of vestibular evoked myogenic potentials in patients with Meniere's disease. J Vestib Res 2013;23(4-5):249-57.

60. Inoue A, Egami N, Fujimoto C, et al. Vestibular evoked myogenic potentials in vestibular migraine: Do they help differentiating from Meniere's disease? Ann Otol Rhinol Laryngol 2016;125(11):931-7.

61. Lamounier P, de Souza TSA, Gobbo DA, et al. Evaluation of vestibular evoked myogenic potentials (VEMP) and electrocochleography for the diagnosis of Meniere's disease. Braz J Otorhinolaryngol 2017;83(4): 394-403.

62. Murofushi T, Nakahara H, Yoshimura E, et al. Association of air-conducted sound oVEMP findings with CVEMP and caloric test findings in patients with unilateral peripheral vestibular disorders. Acta Otolaryngol 2011;131(9): 945-50.

63. Taylor RL, Bradshaw AP, Halmagyi GM, et al. Tuning characteristics of ocular and cervical vestibular evoked myogenic potentials in intact and dehiscent ears. Audiol Neurootol 2012;17(4):207-18.

64. Karimnejad K, Czerny MS, Lookabaugh S, et al. Gender and laterality in semicircular canal dehiscence syndrome. J Laryngol Otol 2016;130(8):712-6.

65. Thomas K, Harrison MS. Long-term follow up of 610 cases of Meniere's disease. Proc R Soc Med 1971; 64(8):853-7.

66. Paparella MM, Griebie MS. Bilaterality of Meniere's disease. Acta Otolaryngol 1984;97(3-4):233-7.

67. Wladislavosky-Waserman P, Facer GW, Mokri B, et al. Meniere's disease: a 30-year epidemiologic and clinical study in Rochester, Mn, 1951-1980. Laryngoscope 1984;94(8):1098-102.

68. Kitahara M, Matsubara H, Takeda T, et al. Bilateral Meniere's disease. Adv Otorhinolaryngol 1979;25:117-21.

69. Tsuji K, Velázquez-Villaseñor L, Rauch SD, et al. Temporal bone studies of the human peripheral vestibular system. Meniere's disease. Ann Otol Rhinol Laryngol Suppl 2000;181:26-31.

70. House JW, Doherty JK, Fisher LM, et al. Meniere's disease: prevalence of contralateral ear involvement. Otol Neurotol 2006; 27(3):355-61.

71. Chaves AG, Boari L, Lei Munhoz MS. The outcome of patients with Menieres disease. Braz J Otorhinolaryngol 2007;73(3):346-50.

72. Salviz M, Yuce T, Karatas A, et al. Diagnostic value of frequency-associated vestibular-evoked myogenic potential responses in Meniere's disease. Audiol Neurootol 2015;20(4):229-36.

73. Zhu Y, McPherson J, Beatty C, et al. Cervical VEMP threshold response curve in the identification of Meniere's disease. J Am Acad Audiol 2014;25(3):278-88; quiz 302-3. 
74. Fife TD, Colebatch JG, Kerber KA, et al. Practice guideline: Cervical and ocular vestibular evoked myogenic potential testing: Report of the Guideline Development, Dissemination, and Implementation Subcommittee of the American Academy of Neurology. Neurology, 2017.

75. Lin MY, Timmer FC, Oriel BS, et al. Vestibular evoked myogenic potentials (VEMP) can detect asymptomatic saccular hydrops. Laryngoscope 2006;116(6):987-92.

76. Minor LB, Solomon D, Zinreich JS, et al. Sound- and/or pressure-induced vertigo due to bone dehiscence of the superior semicircular canal. Arch Otolaryngol Head Neck Surg 1998;124(3):249-58.

77. Carey JP, Minor LB, Nager GT. Dehiscence or thinning of bone overlying the superior semicircular canal in a temporal bone survey. Arch Otolaryngol Head Neck Surg 2000;126(2):137-47.

78. Cloutier JF, Belair M, Saliba I. Superior semicircular canal dehiscence: positive predictive value of highresolution CT scanning. Eur Arch Otorhinolaryngol 2008;265(12):1455-60.

79. Rosowski JJ, Songer JE, Nakajima HH, et al. Clinical, experimental, and theoretical investigations of the effect of superior semicircular canal dehiscence on hearing mechanisms. Otol Neurotol 2004;25(3): 323-32.

80. Ho ML, Moonis G, Halpin CF, et al. Spectrum of Third Window Abnormalities: Semicircular Canal Dehiscence and Beyond. AJNR Am J Neuroradiol 2017;38(1):2-9.

81. Zhou G, Gopen Q, Poe DS. Clinical and diagnostic characterization of canal dehiscence syndrome: a great otologic mimicker. Otol Neurotol 2007;28(7):920-6.

82. CNiesten MEF. Evaluation and management of superior canal dehiscence syndrome. 2014, Universiteit Utrecht. p. Chapter 2: SCD prevalence of superior canal dehiscence following failed stapes surgery.

83. Benamira LZ, Alzahrani M, Saliba I. Superior canal dehiscence: can we predict the diagnosis? Otol Neurotol 2014;35(2):338-43.

84. Govender S, Fernando T, Dennis DL, et al. Properties of $500 \mathrm{~Hz}$ air- and bone-conducted vestibular evoked myogenic potentials (VEMPs) in superior canal dehiscence. Clin Neurophysiol 2016;127(6): 2522-31.

85. Milojcic R, Guinan JJ Jr, Rauch SD, et al. Vestibular evoked myogenic potentials in patients with superior semicircular canal dehiscence. Otol Neurotol 2013;34(2):360-7.

86. Zuniga MG, Janky KL, Nguyen KD, et al. Ocular versus cervical VEMPs in the diagnosis of superior semicircular canal dehiscence syndrome. Otol Neurotol 2013;34(1):121-6.

87. Lopez-Escamez JA, Carey J, Chung WH, et al. Diagnostic criteria for Meniere's disease. J Vestib Res 2015; 25(1):1-7.

88. Hulse R, Biesdorf A, Hörmann K, et al. Peripheral Vestibular Disorders: An Epidemiologic Survey in 70 Million Individuals. Otol Neurotol 2019;40(1):88-95.

89. Minor LB, Schessel DA, Carey JP. Meniere's disease. Curr Opin Neurol 2004;17(1):9-16.

90. Kotimaki J, Sorri M, Aantaa E, et al. Prevalence of Meniere disease in Finland. Laryngoscope 1999; 109(5):748-53.

91. Schessel DA, Minor L, Nedzelski J. Meniere's disease and other peripheral vestibular disorders. In: Otolaryngology Head and Neck Surgery. 1998, Mosby, St. Louis

92. Rauch SD, Merchant SN, Thedinger BA. Meniere's syndrome and endolymphatic hydrops. Double-blind temporal bone study. Ann Otol Rhinol Laryngol 1989;98(11):873-83.

93. Merchant SN, Adams JC, Nadol JB Jr. Pathophysiology of Meniere's syndrome: are symptoms caused by endolymphatic hydrops? Otol Neurotol 2005;26(1):74-81.

94. Taylor RL, Zagami AS, Gibson WP, et al. Vestibular evoked myogenic potentials to sound and vibration: characteristics in vestibular migraine that enable separation from Meniere's disease. Cephalalgia 2012; $32(3): 213-25$. 



\section{Chapter 1.2}

Aims and outline 



\section{Aims and outline}

This thesis aims to address the shortcomings and issues of the cVEMP that were discussed in the general introduction to improve its clinical use.

\section{Toward optimizing cervical vestibular evoked myogenic potentials}

Chapter 2.1 Calculating VEMP inhibition depth with a generic template

The use of the relatively new cVEMP metric VEMP inhibition depth in a clinical population requires the use of a generic template, which has not been used before. The performance of VEMP inhibition depth, derived using a subject-specific template versus VEMP inhibition depth derived using a generic template was compared.

Chapter 2.2 Normalization reduces the need for strong muscle contraction

The effect of muscle contraction strength on cVEMP metrics was assessed to determine the muscle contraction range within which the cVEMP can reliably be assessed using normalization techniques.

\section{Clinical use of cVEMP in patients with superior semicircular canal dehiscence syndrome}

Chapter 3.1 Combining air-bone gap and cVEMP thresholds to improve diagnosis of superior canal dehiscence syndrome

A novel approach, combining the commonly tested low-frequency audiometric air-bone gap and cVEMP thresholds, was developed to improve screening for superior semicircular canal dehiscence syndrome (SCD).

Chapter 3.2 Audiometric and cVEMP thresholds show little correlation with symptoms in superior semicircular canal dehiscence syndrome

The relationship between objective audiometric and vestibular tests and subjective patient symptoms was evaluated in patients with superior canal dehiscence syndrome.

Chapter 3.3 $2000 \mathrm{~Hz}$ tone bursts improve the detection of superior semicircular canal dehiscence syndrome

It was prospectively investigated which cVEMP metric and frequency best differentiate healthy ears from SCD ears.

\section{Clinical use of cVEMP in Menière's disease patients}

Chapter 4.1 Cervical vestibular evoked myogenic potentials in Menière's disease: A comparison of response metrics 
To assess the value of different cVEMP metrics in differentiating Menière's disease patients from age matched controls, these groups were prospectively included and tested with a specific cVEMP protocol.

Chapter 4.2 Predicting development of bilateral Menière's disease based on CVEMP threshold and tuning

A group of patients, previously diagnosed with unilateral Menière's disease, was contacted to investigate who developed bilateral disease. Their cVEMP test from $\geq 5$ years ago was analyzed to investigate whether the old CVEMP data was predictive of who would develop bilateral disease. 




\section{Chapter

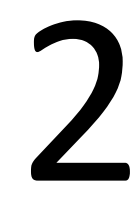

Toward optimizing cervical vestibular evoked myogenic potentials 



\section{Chapter}

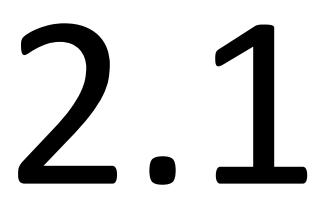

\section{Calculating VEMP inhibition depth with}

a generic template

KS Noij

MJ van Tilburg

BS Herrmann

P Marciniak

SD Rauch

JJ Guinan Jr. 


\section{Abstract}

\section{Objectives}

Cervical vestibular evoked myogenic potentials (cVEMP) indirectly reveal the response of the saccule to acoustic stimuli through the inhibition of the sternocleidomastoid muscle electromyographic (EMG) response. VEMP inhibition depth (VEMPid) is a recently developed metric that estimates the percentage of saccular inhibition. VEMPid provides both normalization and better accuracy at low response levels than amplitude-normalized cVEMPs. Hopefully, VEMPid will aid in the clinical assessment of patients with vestibular pathology. To calculate VEMPid a template is needed. In the original method, a subject's own cVEMP was used as the template, but this method can be problematic in patients who do not have robust cVEMP responses. We hypothesize that a "generic" template, created by assembling cVEMPs from healthy subjects, can be used to compute VEMPid, which would facilitate the use of VEMPid in subjects with pathological conditions.

\section{Design}

A generic template was created by averaging CVEMP responses from six normal subjects. To compare VEMPid calculations using a generic versus a subject-specific template, cVEMPs were obtained in 40 healthy subjects using 500, 750 and $1000 \mathrm{~Hz}$ tone bursts at sound levels ranging from 98 to $123 \mathrm{~dB}$ peSPL. VEMPids were calculated both with the generic template and with the subject's own template. The ability of both templates to determine whether a cVEMP was present or not was compared with receiver operating characteristic $(\mathrm{ROC})$ curves.

\section{Results}

No significant differences were found between VEMPid calculations using a generic template versus using a subject-specific template for all frequencies and sound levels. Based on the ROC curves, the subject-specific and generic template did an equally good job at determining threshold. Within limits, the shape of the generic template did not affect these results.

\section{Conclusions}

A generic template can be used instead of a subject-specific template to calculate VEMPid. Compared to cVEMP normalized by electromyographic amplitudes, VEMPid is advantageous because it averages zero when there is no sound stimulus and it allows the accumulating VEMPid value to be shown during data acquisition as a guide to decide when enough data has been collected. 


\section{Introduction}

The cervical vestibular evoked myogenic potential (cVEMP) arises from the saccule in response to acoustic or mechanical stimuli that leads to inhibition of cervical muscle electromyographic (EMG) activity and can be measured using surface electrodes on the ipsilateral sternocleidomastoid muscle (SCM) (1). Clinically, cVEMPs are mostly used to assess patients with Menière's disease (MD) and superior semicircular canal dehiscence syndrome (SCD) (2-6).

A major shortcoming in CVEMP measurements is that the results vary considerably from one subject to the next, which leads to overlap in amplitude and threshold between healthy and pathologic subjects. Many factors, including subject age, muscle contraction intensity, muscle mass and electrode position influence cVEMP amplitudes (7-9). Since saccular activation inhibits SCM EMG activity, the SCM must be tonically contracted for the inhibition to be measured. CVEMP peak-to-peak amplitude (VEMPpp) grows almost linearly with muscle contraction amplitude so that variation in muscle contraction produces variation when using VEMPpp as the cVEMP metric. However, this variation can be compensated for by using normalization techniques, for example, by dividing the peak-to-peak VEMP amplitude by the overall EMG amplitude to yield a normalized cVEMP (VEMPn) (10-12). An alternate cVEMP metric, the VEMP inhibition depth (VEMPid) also provides normalization and has the advantage that its value averages zero when there is no sound, whereas VEMPpp and VEMPn have floor values set by the noise in the EMG $(13,14)$. There is evidence that VEMPid is more sensitive in detecting changes in saccular function and may be better at detecting threshold than VEMPn (13). How much this potential advantage of VEMPid can be realized in a patient population remains to be determined.

Prakash et al. (13) hypothesized that the linear growth of cVEMP with EMG amplitude exists because any particular saccular response causes a certain percentage of SCM motoneuron responses to be inhibited, and this percentage does not change with EMG amplitude (i.e. an increase in motor drive that increases the EMG and the resulting cVEMP amplitude does not change the fraction of motoneuron responses that are inhibited). Using a computational model of the SCM motor control system, Prakash et al. (13) derived a method to estimate the percentage inhibition of the motoneuron responses, the VEMPid, based on a series of individual cVEMP responses. VEMPid is calculated using a template correlation method that resembles the use of a matched filter for the detection of a signal (the template) in noise (13). The EMG waveform from each cVEMP response is compared to the template, which yields a single number, the template correlation value (TCV), that estimates how much the waveform resembles the template. VEMPid is then calculated from the set of TCVs obtained from the recordings that would yield a cVEMP.

To calculate VEMPid, a template is needed. In the original VEMPid method, the template was determined from the subjects' own cVEMP, measured at a high sound 
level, in the same ear and at the same sound frequency (13). This requires an individual to have a robust cVEMP response in order to use the VEMPid metric. This requirement is problematic in a clinical setting where patients with vestibular disease may or may not have a robust cVEMP that is usable as a template. One possibility is that in patients with suspected unilateral disease (MD or SCD), the template could be derived from the contralateral and presumed healthy ear. Unfortunately, the assumption that the contralateral ear is healthy is not always met, as indicated by the decreased cVEMP amplitudes and elevated thresholds of the presumed unaffected ear in MD patients (2). In fact, bilateral disease occurs in 9 to $50 \%$ in MD patients (15-21) and in $25.5 \%$ in SCD patients (22). The requirement of a good subject-specific template requires the clinician to determine whether a cVEMP is present or absent at high levels using a subjective judgment of whether the waveform obtained is similar to what is expected for a CVEMP response, in other words, using the clinician's mental template of a cVEMP response. One of our goals in the present work is to reduce the role of subjective judgments in assessing cVEMP.

A possible solution for the template problem in VEMPid calculations is the use of a "generic" template. A generic template could be created from a cVEMP model or from cVEMP responses from healthy individuals. It is unknown, however, how well this will work, or whether using a generic template will produce substantially different VEMPids compared to using the subjects' own template. If a generic template can be used, its use will enable VEMPid to be obtained in patients in whom the use of a subject-specific template is not possible or desirable. The purpose of the present study is to compare VEMPid calculated using a generic template to VEMPid calculated using a subjectspecific template in healthy individuals.

\section{Materials and methods}

\section{Subjects}

Two groups of healthy subjects were included. cVEMPs were obtained from both groups using the same cVEMP recording method but somewhat different stimulus parameters. Which group's data were used in each data analysis was determined by which best suited the objectives of the study analysis. The first group included 20 healthy subjects (11 women, mean age: 29 years, range: 20 to 48 years), and was previously described in van Tilburg et al. (11). The second group also included 20 healthy subjects (10 women, mean age: 28.3 years, range: 21 to 36 years). Exclusion criteria were: a history of balance issues, hearing loss, any neurologic disease, or an airbone gap ( $A B G$ ) of $>10 \mathrm{~dB}$. To verify that no $A B G$ was present, all subjects received air and bone audiograms before cVEMP testing. To monitor the influence of cVEMP on cochlear status, distortion product otoacoustic emissions (DPOAE) were screened 
immediately before and after testing. All subjects passed DPOAE screening before and after cVEMP testing. This study was approved by the Human Studies Committee of the Massachusetts Eye and Ear Infirmary (protocol No. 13-097H, principal investigator:

S.D. Rauch).

\section{cVEMP acquisition}

Subjects sat up straight and contracted their SCM muscle by turning their head away from the stimulated ear. EMG activity was recorded from four surface electrodes: a positive electrode on the middle belly of each SCM, a reference electrode on the sternum at the point where the SCM is attached to the manubrium and a ground electrode on the forehead (midline). The EMG was monitored throughout testing and subjects maintained root mean square (rms) EMGs of $>65 \mu \mathrm{V}$. EMG activity was amplified, bandpass filtered and sampled at $50 \mathrm{kHz}$ using a 16-bit analog to digital converter (National Instruments).

The saccule was excited by tone bursts presented by circumaural headphones (Telephonics TDH-49) at a repetition rate of $13 / \mathrm{s}$ with two-cycle rise and fall times (Blackman gating function) and no plateau. In the first subject group, 3 cVEMPs were recorded on each side using 500, 750 and $1000 \mathrm{~Hz}$ tone bursts at $123 \mathrm{peSPL}$, and single cVEMPs were also recorded for $500 \mathrm{~Hz}$ at 98, 103, 108, 113 and 118 peSPL (123 dB peSPL equals $90 \mathrm{~dB} \mathrm{nHL}$ ). For the second subject group, single cVEMPs were recorded on each side using 500, 750 and $1000 \mathrm{~Hz}$ tone bursts at 93, 103, 113 and $123 \mathrm{~dB}$ peSPL and two additional recordings were obtained without any stimulus: the "no sound condition" (i.e. subjects contracted their SCM, and EMGs were recorded, but no sound was provided). For all recordings, 200 to 300 EMG responses were obtained and analyzed. The presentation order was randomized for sound level, frequency and side.

\section{Templates}

In the original derivation of the VEMPid calculation, the template was subject specific and was obtained from a separate cVEMP recording acquired the highest sound level (123 dB peSPL) from the same ear and tone frequency as the CVEMPs to be measured. The recorded waveform was then trimmed, i.e. the waveform amplitude was set to zero at times before and after the VEMP response. Originally this trimming was done by eye for each template (13). Later this subjective step was replaced by using boundaries that were set at fixed times chosen for each tone frequency so that the boundaries extended early and late enough to include the peaks of cVEMP waveforms of all different latencies (14). Although this method was objective and easily-applied, it had the drawback that the time boundaries were wide and usually included noisy, nonVEMP-response regions at the edges. To get a subject-specific VEMPid for the present paper, we used the objective, wide, fixed-window method and the first CVEMP obtained at $123 \mathrm{~dB}$ peSPL for that frequency and side. Since a VEMPid calculation is not 
valid when a template is applied to the CVEMP response used to create the template, an additional cVEMP recording at the highest level was needed to get a VEMPid for that intensity level in the data analyses.

To obtain the generic template we selected waveforms from 1 ear of 6 healthy subjects with typical clean-looking cVEMP waveforms. The individual cVEMPs varied in latency (Figure 2.1.1A). We aligned them in time by setting their zero-crossing time to $16 \mathrm{~ms}$ (Figure 2.1.1B). Sixteen milliseconds is the average zero-crossing time of CVEMP responses for subjects in group 2 with cVEMP latency expressed relative to the soundstimulus peak, which removes the CVEMP latency variation from the different stimulus rise-times at different frequencies (11). We averaged the aligned waveforms and trimmed the edges so that only the central, cVEMP waveform was included. This trimmed average waveform was used as the base generic template (Figure 2.1.1B, thick trace). To enable tests with generic waveforms of different widths, the base template (displayed by the thick black line in Figure 2.1.1C) was stretched and shrunk from half its width to twice its width along the time axis yielding a family of generic waveforms (Figure 2.1.1C).

\section{VEMPid calculation}

Several steps are required to calculate VEMPid (see Prakash 2015 for details) (13). These steps include normalizing the template so that its rms value summed across time is unity, and then calculating the correlations (or inner products) of the template with each single cVEMP EMG trace in the cVEMP response. These single cVEMP traces, averaged together, make up the averaged cVEMP response. Each correlation produces a template correlation value, or TCV, a scalar value representing how closely the single cVEMP trace resembles the template (Figure 2.1.2A, B). The SCM motor-control model showed that the fractional inhibition of SCM motoneurons (the inhibition depth, or VEMPid) was a constant (0.2) times the mean of the TCVs divided by the standard deviation of the TCVs $(13,23)$. Using this result, we calculated the inhibition depth as: VEMPid $=0.2 \times$ Mean (TCV) $/$ std(TCV).

A subject-specific template has the cVEMP latency of the subject, but the generic template does not contain latency information. To determine at what latency the generic template should be set, a sliding-template method was used on a set of CVEMP responses obtained at the highest sound level for each side and frequency. This sliding template method calculated correlations at every $0.1 \mathrm{~ms}$ from $4 \mathrm{~ms}$ before the base latency (which is the zero-crossing latency of $16 \mathrm{~ms}$, plus the stimulus-frequencylatency-correction of 2000/frequency) to $7 \mathrm{~ms}$ after the base latency (Figure 2.1.2B). 


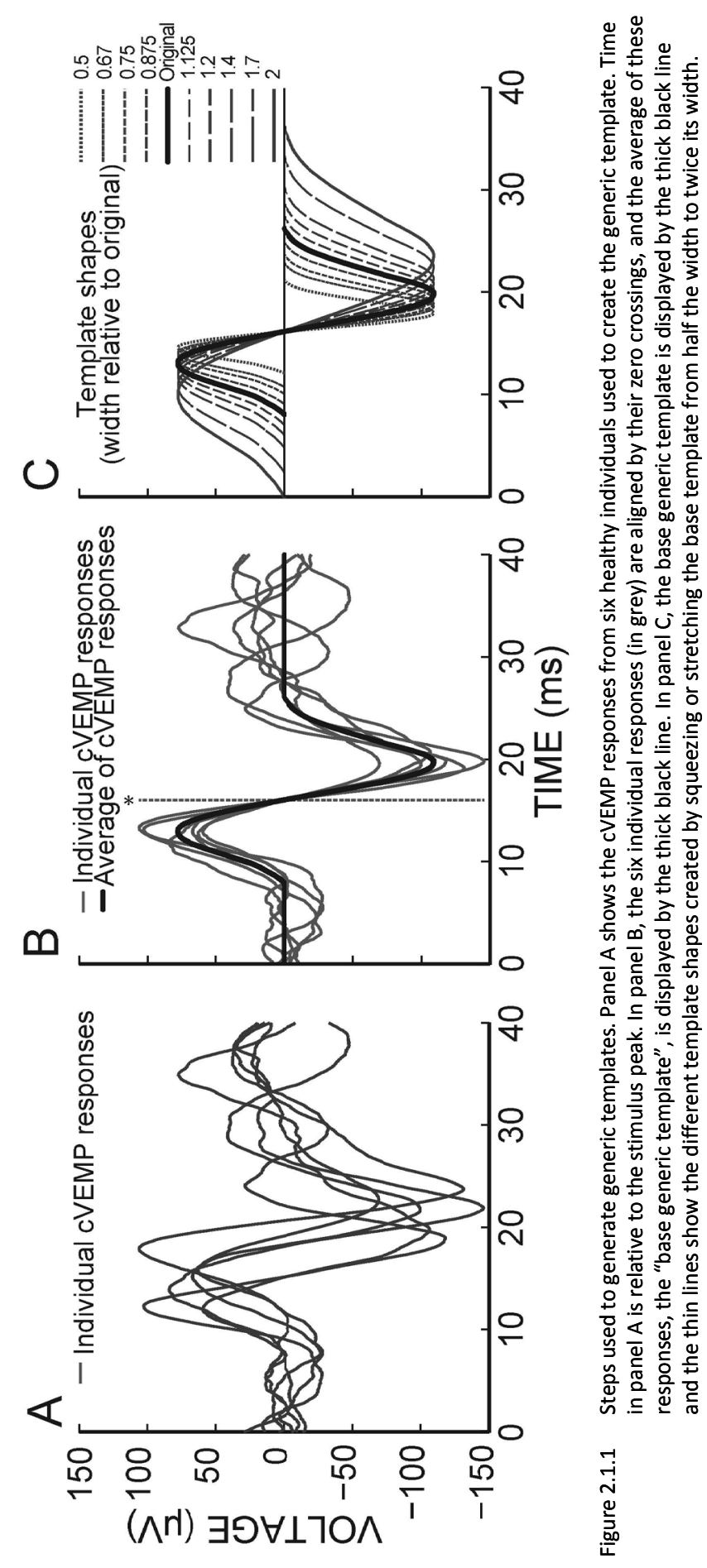


This time range insured that the template covered the range of latencies found in normal cVEMP waveforms. At every $0.1 \mathrm{~ms}$, the correlation of the template with each cVEMP response was calculated as it was using a subject-specific template, namely the sliding-template-TCV $=\left(\left(a_{1} \cdot b_{1}\right)+\left(a_{2} \cdot b_{2}\right)+\ldots+\left(a_{n} \cdot b_{n}\right)\right)$ (Figure 2.1 .2$)$. At every $0.1 \mathrm{~ms}$ step, the sliding-template-TCVs were used to calculate a sliding-template-ID using the standard VEMPid formula. The template latency that produced the highest slidingtemplate-ID was used as the template latency for the calculation of VEMPid for all the other cVEMP data sets obtained for the same side and frequency (i.e. the generic template was used at this latency; Figure 2.1.2C, D).

\section{Analyses}

Full factorial analyses of variances (ANOVAs) were performed to determine the effect of template type (subject-specific versus generic) on VEMPid. For group 1 data at $123 \mathrm{~dB}$ peSPL, template, frequency and side were considered fixed factors and the subject was considered a variable factor. Since no significant differences due to the side of recording were revealed by ANOVA ( $F=1.986, p=0.159)$, data from both ears were pooled for subsequent analysis and in the figures. For group 1, lower sound levels (98 to $118 \mathrm{~dB}$ peSPL) were only available at $500 \mathrm{~Hz}$, and a second ANOVA was performed to evaluate the effect of the subject-specific versus the generic template across sound levels. Template, sound level and side were considered fixed factors and the subject was considered a variable factor. Again, no significant differences due to side of recording were revealed for the $500 \mathrm{~Hz}$ data at lower stimulus levels $(F=1.883$, $p=0.171$ ). Therefore, data from both ears were pooled for figures and subsequent analysis. For group 2, data below $123 \mathrm{~dB}$ peSPL were available at 500, 750 and $1000 \mathrm{~Hz}$. To evaluate the effect of template type on VEMPid for all frequencies at these lower sound levels (93 to $113 \mathrm{~dB}$ peSPL) a third ANOVA was performed. In these, template type, frequency, sound level and side were considered fixed factors and the subject was considered a variable factor. 


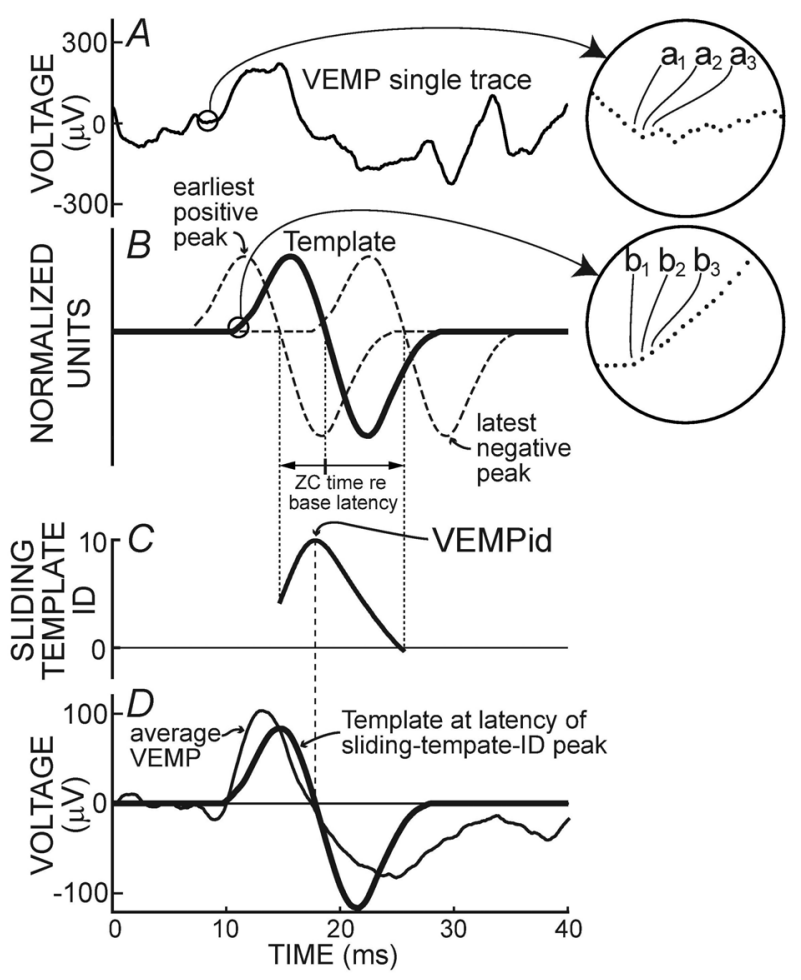

Figure 2.1.2 A display of the calculations used to set the latency of the generic template for a VEMPid calculation using a generic template. Panel A shows an example waveform showing a single VEMP-response EMG. The expanded view shows the labeling of the first three points used to get the template correlation value $\left(\operatorname{TCV}=\left(\left(a_{1} \cdot b_{1}\right)+\left(a_{2} \cdot b_{2}\right)+\ldots+\left(a_{n} \cdot b_{n}\right)\right)\right)$ when the template is at its earliest search latency. In panel $B$ the solid line shows the template at the base latency (i.e. the zero crossing (ZC) is at 16 ms plus the stimulus-frequency-latency-correction of 2000/frequency, $f=750 \mathrm{~Hz})$. The expanded view shows the labeling of the first three points $\left(b_{1}, b_{2}, b_{3}\right)$ used to get the TCV at the base latency. Dashed lines show the template at the earliest and latest search latencies. A sliding template ID (=0.2 $\times$ mean (TCV) / std (TCV)), was calculated for each of the template latencies from the earliest to the latest, in $0.1 \mathrm{~ms}$ steps from -4 to $+7 \mathrm{~ms}$ relative to the base template latency. This sliding range makes the earliest template be aligned with the earliest observed CVEMP positive peak and the latest template be aligned with the latest observed CVEMP negative peak. Panel $C$ shows the sliding template ID versus the latency of the template zero crossing over the sliding range. VEMPid is the peak value of the sliding template ID and VEMPid latency is the latency of the sliding template ID peak (dashed line from C to D). Panel $D$ shows the generic template (thick line in panel D) displayed at the latency of the sliding-template-ID peak together with the average CVEMP (thin line in panel D) for the data that were used. 
To determine how well each template can distinguish a sound-evoked cVEMP from noise, Cohen's $d$ effect size was calculated. Cohen's $d$ is the difference between a group mean $\left(\mu_{1}\right)$ at a certain sound level, and the corresponding no-sound mean $\left(\mu_{2}\right)$, divided by the combined standard deviation of the two groups $\left(\sigma_{1,2}\right)$.

$$
\text { Cohen's } d=\frac{\mu_{1}-\mu_{2}}{\sigma_{1,2}}
$$

Measurements with no sound stimulus were only obtained from group 2, therefore Cohen's $d$ was only calculated for group 2 data. Statistical analyses were performed using MatLab (version 2013a) and SPSS (version 22.0; Chicago, IL). $P$ values of $<0.05$ were considered statistically significant.

\section{Threshold assessment by audiologists}

The ability of VEMPid to determine threshold was assessed by comparing VEMPid values to the CVEMP thresholds determined visually by audiologists, which is how threshold is determined in most clinical settings. Three audiologists, with at least 5 years of experience assessing cVEMPs, independently determined the threshold from each sound-level series of cVEMPs. The use of a subject-specific template, a generic template and different template shapes were assessed on their ability to determine whether a cVEMP was present, or not, by comparing their receiver operating characteristic (ROC) curves.

\section{Results}

VEMPids calculated using a subject-specific versus the base generic template were very similar for each set of cVEMP data. For differences due to template type, ANOVAs showed no significant difference for: group 1 data at $123 \mathrm{~dB}$ peSPL and 500, 750 and $1000 \mathrm{~Hz}(\mathrm{~F}=0.051, p=0.821)$, for group $1,500 \mathrm{~Hz}$ data at lower sound levels (98 to $118 \mathrm{~dB}$ peSPL) $(\mathrm{F}=0.021, p=0.886)$ and for group 2 data at 500,750 and $1000 \mathrm{~Hz}$ at all sound levels below $123 \mathrm{~dB}$ peSPL $(F=0.066, p=0.798)$. As expected, significant effects were found for sound level (using $500 \mathrm{~Hz}$ data only for group 1) and frequency (using $123 \mathrm{~dB}$ peSPL data only for group 1). Such differences have been reported before, and are not of interest here $(2,11,24)$. In addition, controlling for frequency, no significant effect of sound level was found on the latencies of P1, zero crossing, or N1 ( $F=1.463$, $p=0.201 ; \mathrm{F}=0.991, p=0.423 ; \mathrm{F}=1.304, p=0.261$, respectively). The similarities of VEMPid calculated with the base generic template versus a subject-specific template are shown for the group 1 data as a scatter plot of individual values (Figure 2.1.3), and as averages at each sound level and frequency tested (Figure 2.1.4). The correlations 
between the individual VEMPid values from a subject-specific versus from the generic template were very high ( $r=0.989$ for group 1 data, $r=0.972$ for group 2 data).

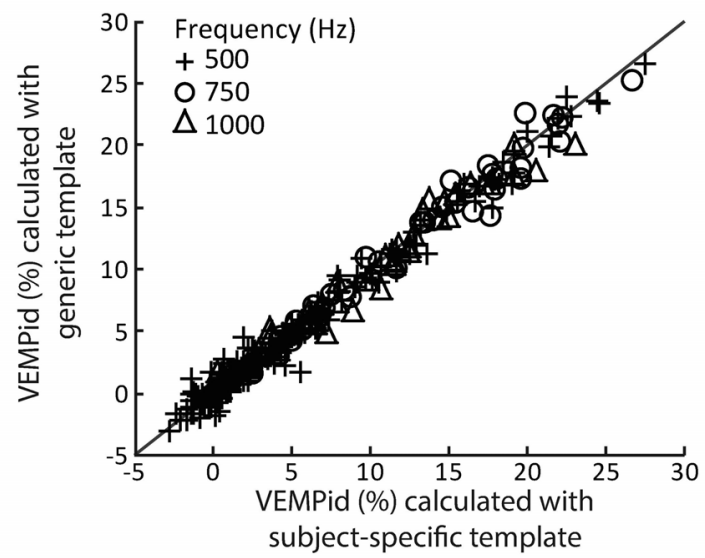

Figure 2.1.3 VEMPid calculated with a subject-specific template plotted against VEMPid calculated with the generic template for 500,750 and $1000 \mathrm{~Hz}$ using group 1 data. Stimulus levels include 98 to 123 $\mathrm{dB}$ peSPL for $500 \mathrm{~Hz}$ and $123 \mathrm{~dB}$ peSPL at 750 and $1000 \mathrm{~Hz}$. There is a good correlation between the two templates (correlation coefficient of 0.989).

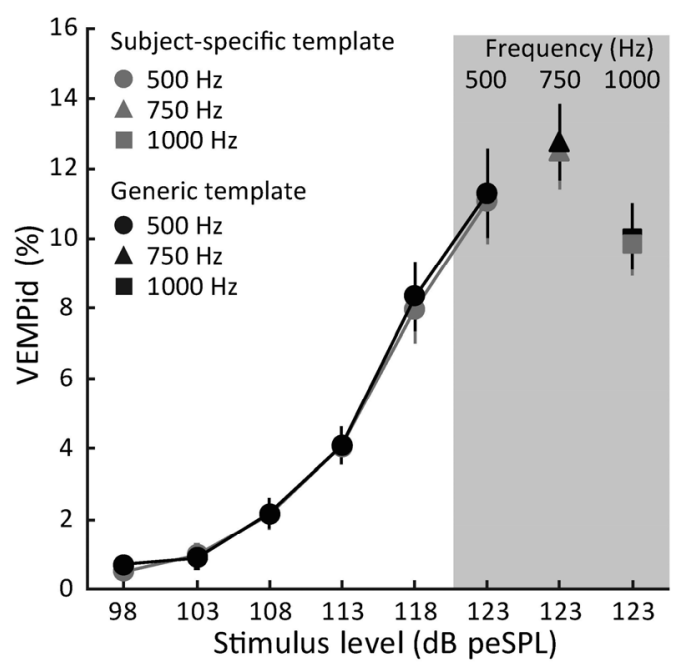

Figure 2.1.4 Average VEMPid calculated with the subject-specific template (grey symbols) and the generic template (black symbols) using group 1 data. Error bars indicate the standard error of the mean. VEMPids calculated with both templates are very similar. The shaded area of the graph highlights the comparison of the data at $123 \mathrm{~dB}$ peSPL across stimulus frequencies; the nonshaded area of the graph highlights the comparison across stimulus level. 
To determine the effect of template width, VEMPid was calculated using each of the family of generic templates in Figure 2.1.1C. The second subject group was used for this analysis because this group had no-sound runs available which allowed for the calculation of Cohen's $d$ (which measures how well the CVEMP can be distinguished from noise). The results show that at $123 \mathrm{~dB}$ peSPL slightly higher VEMPid values were obtained with narrower (i.e. a width smaller than the width of the original template) templates, while slightly higher Cohen's $d$ values were obtained with slightly wider templates at $500 \mathrm{~Hz}$. Cohen's $d$ was relatively flat at $750 \mathrm{~Hz}$ and at $1000 \mathrm{~Hz}$ Cohen's $d$ is slightly higher for the narrower templates (Figure 2.1.5D-F). Overall, small changes in template width made little difference in VEMPid or Cohen's $d$, but large changes in width produced distinctly worse results (Figure 2.1.5). Since it does not appear particularly advantageous to use a different template width, we decided to continue using the base template made from averaging the CVEMP waveforms from the 6 subjects (Figure 2.1.1B).
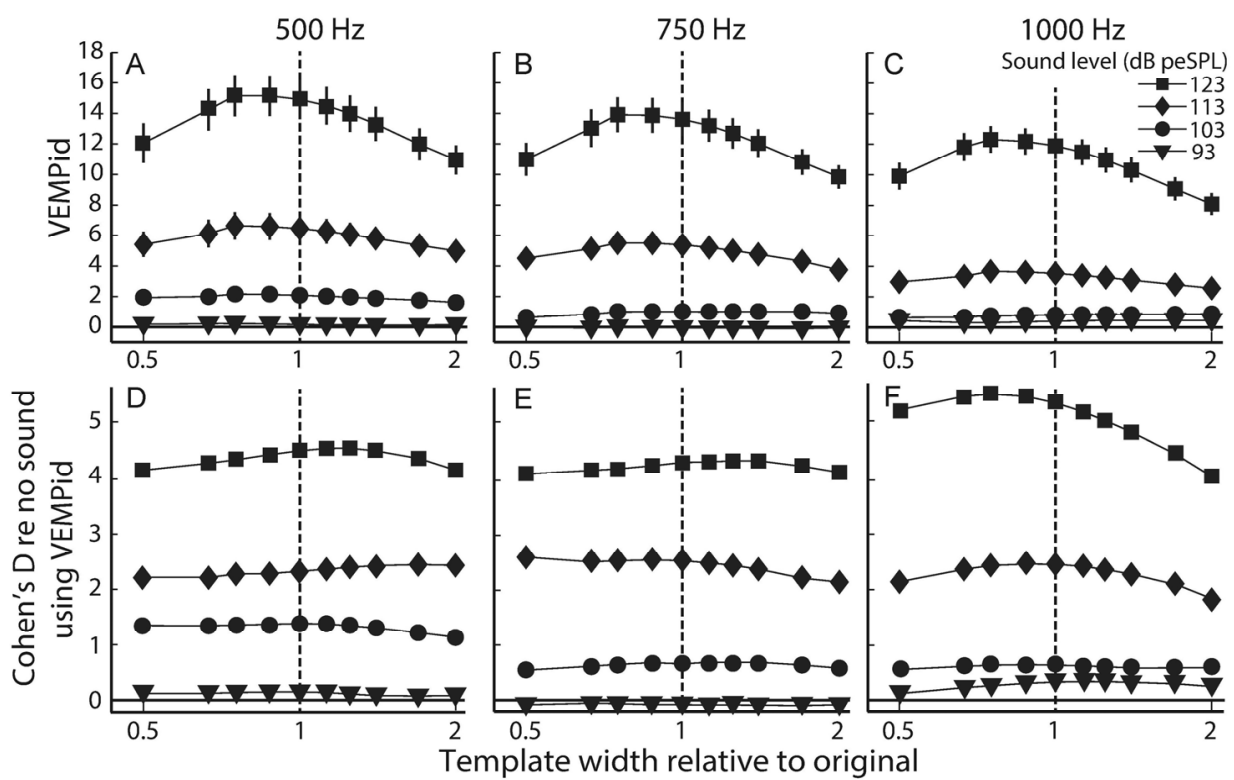

Figure 2.1.5 VEMPids calculated with the different template widths, from half to twice the width of the original template at, 93, 103, 113 and $123 \mathrm{~dB}$ peSPL. The three upper panels show results for 500 (A), 750 (B) and $1000 \mathrm{~Hz}$ (C). Cohen's $d$ is displayed in the lower three panels for 500 (D), 750 (E) and $1000 \mathrm{~Hz}$ (F) calculated with the different template widths, from half to twice the width of the original template at, 93, 103, 113 and $123 \mathrm{~dB}$ peSPL. For all three frequencies VEMPid and Cohen's $d$ are not affected much by slight changes in template width, but large deviations from the original template give a substantial decrease in VEMPid. 
An important issue is whether using the generic template versus a subject-specific template affects how cVEMP thresholds can be determined. As the current "gold standard" for determining cVEMP thresholds, we used the thresholds visually assessed by three audiologists. In 462 of 542 cVEMPs ( $500 \mathrm{~Hz}$ : 155 out of 185; $750 \mathrm{~Hz}$ : 158 out of 179; $1000 \mathrm{~Hz}$ : 149 out of 178), all three audiologists agreed on the presence or absence of the waveform. ROC curves from the $500 \mathrm{~Hz}$ data (i.e. subject, side and frequency) when all three audiologists agreed are shown in Figure 2.1.6 along with a table of the areas under the ROC curves (AUCs) for 500, 750 and $1000 \mathrm{~Hz}$. These results show that VEMPid is an accurate way to determine threshold for 500, 750 and $1000 \mathrm{~Hz}$ (Figure 2.1.6). The accuracy in determining threshold is very similar when using a subjectspecific versus a generic template for all frequencies (Figure 2.1.6). At all frequencies, the area under the ROC curves was $>0.93$.

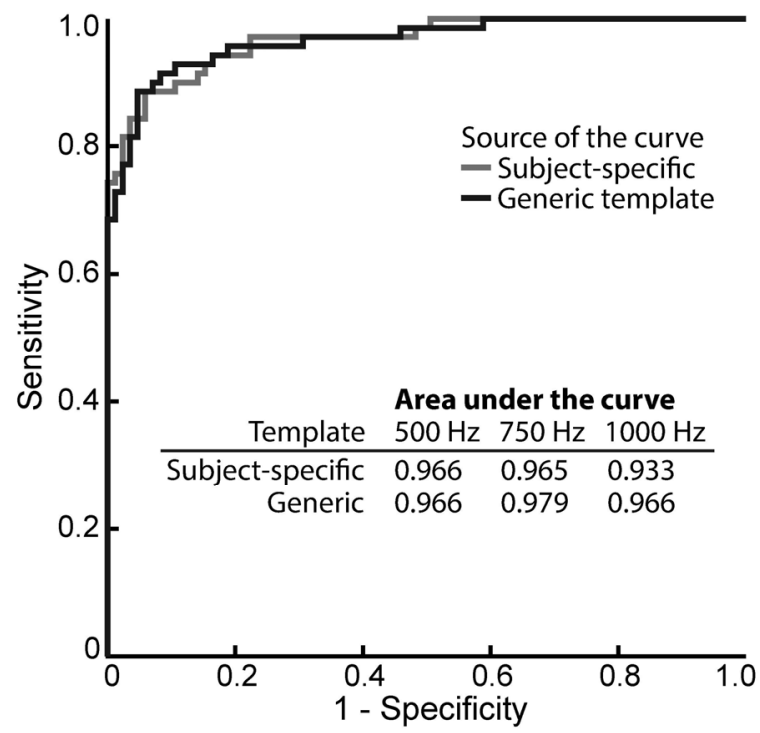

Figure 2.1.6 ROC curves for $500 \mathrm{~Hz}$, group 1 data showing that VEMPid calculated with the subject-specific (grey) and the generic template (black) do an equally good job at determining threshold compared to the subjective judgment of 3 audiologists. The sensitivity on the $y$-axis is equivalent to the true positive rate whereas 1-specificity is the false positive rate.

\section{Discussion}

In this study, we compared the performance of VEMPid derived using a subject-specific template versus VEMPid derived using a generic template. We found only very small differences that were not statistically significant in the performance of VEMPid derived 
using either template, suggesting that a generic template could be used clinically instead of a subject-specific template in the calculation of VEMPid. In particular, a template does not need to duplicate the exact variations in the CVEMP shape shown in a subject (e.g. variations that might be due to differences in muscle size or the depth of overlying fat) for the template to produce a VEMPid that is similar to the VEMPid from the subject-specific template (Figures 2.1.3 and 2.1.4). The use of a generic template to calculate VEMPid is an important step in being able to use VEMPid in the assessment of patients with vestibulopathy in whom the use of a subject-specific template has limitations.

At first it seems surprising that a generic template can do just as well as the subjectspecific template. The closer the template shape is to the underlying signal shape, the higher the correlation will be. The generic template does not include the individual details that were included in a subject-specific template so one would expect it would produce a smaller VEMPid. However, the subject-specific template was obtained with a wide waveform-trimming window that included noise at the edges, whereas the generic template has no noise at its edges. The similar VEMPid values obtained with the generic and subject-specific templates suggests that these two factors offset each other.

One might question whether a template made by averaging cVEMPs from many more than 6 subjects might do a better job. However, because differences between VEMPid calculated with the generic versus the subject specific template are small (Figures 2.1.3 and 2.1.4) and small changes in template width had little effect on VEMPid and Cohen's $d$ (Figure 2.1.5), it seems unlikely that a template made from averaging more subjects would do significantly better. Furthermore, using threshold judgments by audiologists as a standard, the subject-specific and the generic template do an equally good job at determining threshold (i.e. they yield high areas under the ROC curves) for all frequencies (Figure 2.1.6).

Because the generic template does not contain latency information, the timing of the generic template has to be adjusted for every subject. Measuring VEMPid with the template at a fixed latency is a key factor in making the VEMPid equal to zero when there is no sound stimulus. If instead, a sweep across a range of delays was done at every level and the highest sliding-template-ID were chosen (as in Figure 2.1.2), the result would always be positive and the resulting VEMPid would have a floor value set by the noise, similar to the VEMPpp and VEMPn metrics. Using a latency set by the value obtained at the highest sound level works because we found little latency variation between cVEMP responses as a function of sound level. Reports on the effect of sound level on latency are variable as well as the methods used in these studies $(12,25,26)$. However, Rahne et al. (26) investigated the effect of multiple variables on latency (including sound level and multiple methods of muscle contraction) and found no significant effect of either of these factors. A reason to be careful with matching latencies is that most cVEMPs show later potentials $(n 34, p 44)$ that may originate from 
cochlear afferents and these later potentials should not be mistaken for a vestibular response (1). Overall, the generic template reduces the limitations of using VEMPid in vestibulopathic patients. However, how to use VEMPid when there is no CVEMP response to determine the generic-template latency is problematic and is deferred for later work.

VEMPid from a generic template is useful for measuring cVEMP threshold. A variety of evidence suggests that in some etiologies CVEMP threshold may be a more clinically relevant metric than CVEMP amplitude from a fixed sound level. For example, in patients with unilateral Menière's disease, compared to amplitudes, thresholds have a higher statistical significance in distinguishing between normal, Menière's affected, and Menière's unaffected ears (2). Threshold is also a more effective outcome measure in distinguishing between Migraine Associated Vertigo and Menière's patients and in tracking disease progression (24). Finally, cVEMP thresholds are valuable in evaluating patients with SCD $(4-6,27,28)$. In clinical practice, threshold is determined by a clinician viewing a series of average EMG response waveforms from different sound levels and subjectively determining the lowest sound level at which a cVEMP response waveform can be observed. Potentially VEMPid could provide a more objective method of determining threshold. VEMPid yields a single-number evaluation of each response that averages zero when there is no response. VEMPpp and VEMPn also provide singlenumber evaluations of the averaged EMG response, but they do not average zero when there is no response - they have floor values set by the EMG noise. We plan to determine statistical tests that will enable VEMPid to be used as an objective measure of threshold. However, even before that is done the fact that VEMPid averages zero when there is no response is useful for determining threshold. For instance, if a low VEMPid is obtained at a given sound level, one or more additional runs at this level can be done and their average will tend toward zero only if the sound level is below threshold.

VEMPid using a generic template might also assist in determining the optimal number of sweeps needed in a cVEMP response. Once the generic template latency is determined, VEMPid can be calculated throughout each cVEMP data-acquisition average at the same frequency. Near the beginning of the run, the resulting (temporary) VEMPid jumps around, but as the run progresses the VEMPid value asymptotes (Figure 2.1.7). This type of graph is included in our custom cVEMP clinical software (23). The plateauing of the VEMPid calculation with each additional sweep might assist the audiologists' decision on when sufficient sweeps have been obtained for a reliable cVEMP recording. If so, use of VEMPid could potentially decrease testing time. 

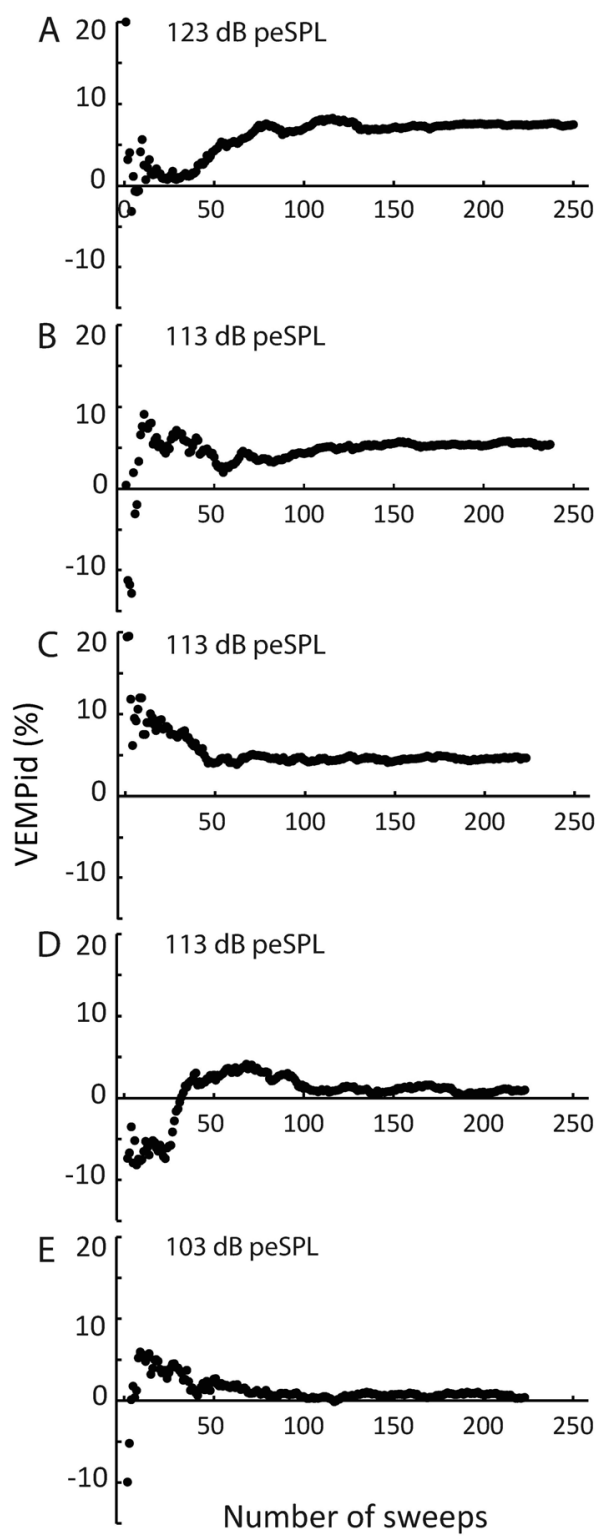

Figure 2.1.7 Examples of how the VEMPid calculation varies in real-time during cVEMP testing as the number of traces in a cVEMP response is increased (shown as implemented in our current clinical software). The first three examples show VEMPid for traces above or at threshold (A-C), while the last 2 panels show examples of VEMPid calculation for traces below threshold ( $D$ and E). In these examples the VEMPid plateaus after different numbers of sweeps. The VEMPid seems to plateau after about 100 sweeps in panel A, D and E, after about 150 sweeps in panel B and about 50 sweeps in panel $C$. Calculations shown in panel $B$ and $C$ are from the same subject, but different sides. The other panels are all from different subjects. 


\section{Conclusion}

A generic template can be used instead of a subject-specific cVEMP template to calculate VEMPid. The resulting VEMPid is useful in determining the cVEMP threshold, and in determining when to stop a data-acquisition run. The use of the generic template reduces the limitations when using VEMPid in patients with pathologies where a subject-specific template can be difficult to identify. 


\section{References}

1. Colebatch JG, Halmagyi GM, Skuse NF. Myogenic potentials generated by a click-evoked vestibulocollic reflex. J Neurol Neurosurg Psychiatry 1994;57(2):190-7.

2. Rauch SD, Zhou G, Kujawa SG, et al. Vestibular evoked myogenic potentials show altered tuning in patients with Meniere's disease. Otol Neurotol 2004;25(3):333-8.

3. Rauch SD, Silveira MB, Zhou G, et al. Vestibular evoked myogenic potentials versus vestibular test battery in patients with Meniere's disease. Otol Neurotol 2004;25:981-6.

4. Benamira LZ, Alzahrani M, Saliba I. Superior canal dehiscence: can we predict the diagnosis? Otol Neurotol, 2014;35:338-43.

5. Mehta R, Klumpp ML, Spear SA, et al. Subjective and objective findings in patients with true dehiscence versus thin bone over the superior semicircular canal. Otol Neurotol 2015;36:289-94.

6. Hunter JB, Patel NS, O'Connell BP, et al. Cervical and ocular VEMP testing in diagnosing superior semicircular canal dehiscence. Otolaryngol Head Neck Surg 2017;156(5):917-923.

7. Welgampola MS, Colebatch JG. Vestibulocollic reflexes: normal values and the effect of age. Clin Neurophysiol 2001;112:1971-9.

8. McCaslin DL, Jacobson GP, Hatton K, et al. The effects of amplitude normalization and EMG targets on cVEMP interaural amplitude asymmetry. Ear Hear 2013;34:482-90.

9. Wei W, Jeffcoat $B$, Mustain W, et al. Frequency tuning of the cervical vestibular-evoked myogenic potential (cVEMP) recorded from multiple sites along the sternocleidomastoid muscle in normal human subjects. J Assoc Res Otolaryngol 2013;14:37-47.

10. Akin FW, Murnane OD, Panus PC, et al. The influence of voluntary tonic EMG level on the vestibularevoked myogenic potential. J Rehabil Res Dev 2004;41(3B):473-80.

11. van Tilburg MJ, Herrmann BS, Guinan JJ Jr, et al. Normalization reduces intersubject variability in cervical vestibular evoked myogenic potentials. Otol Neurotol 2014;35:222-7.

12. Rosengren SM. Effects of muscle contraction on cervical vestibular evoked myogenic potentials in normal subjects. Clin Neurophysiol 2015;126(11):2198-206.

13. Prakash SR, Herrmann BS, Milojcic R, et al. Evaluating Inhibition of Motoneuron Firing From Electromyogram Data to Assess Vestibular Output Using Vestibular Evoked Myogenic Potentials. Ear Hear 2015;36:591-604.

14. Noij KS, Herrmann BS, Rauch SD, Guinan JJ Jr. Toward optimizing VEMP: Normalization reduces the need for strong neck muscle contraction. Audiol Neurotol 2017;22(4-5):282-91.

15. Paparella MM. Pathology of Meniere's disease. Ann Otol Rhinol Laryngol Suppl 1984;112:31-5.

16. Stahle J, Friberg U, Svedberg A. Long-term progression of Meniere's disease. Am J Otol 1989;10:170-3.

17. Yazawa Y, Kitahara M. Bilateral endolymphatic hydrops in Meniere's disease: review of temporal bone autopsies. Ann Otol Rhinol Laryngol 1990;99:524-8.

18. Tsuji K, Velázquez-Villaseñor L, Rauch SD, et al. Temporal bone studies of the human peripheral vestibular system. Meniere's disease. Ann Otol Rhinol Laryngol Suppl 2000;181:26-31.

19. House JW, Doherty JK, Fisher LM, et al. Meniere's disease: prevalence of contralateral ear involvement. Otol Neurotol 2006;27:355-61.

20. Chaves AG, Boari L, Lei Munhoz MS. The outcome of patients with Menieres disease. Braz J Otorhinolaryngol 2007;73(3):346-50.

21. Shojuka H, Watanabe $\mathrm{Y}$, Yagi T, et al. Changes in the characteristics of definite Meniere's disease over time in Japan: a long-term survey by the Peripheral Vestibular Disorder Research Committee of Japan, formerly the Meniere's Disease Research Committee of Japan. Acta Otolaryngol 2009;129:155-60.

22. Karimnejad K, Czerny MS, Lookabaugh S, et al. Gender and laterality in semicircular canal dehiscence syndrome. J Laryngol Otol 2016;130(8):712-6.

23. Prakash SR, Guinan JJ Jr, Herrmann B, Rauch SD. Signal processing in physiological noise. US patent and trademark office, US patent 8,764,676 B2, 2014.

24. van Tilburg MJ, Herrmann BS, Guinan JJ Jr, et al. Serial cVEMP Testing is Sensitive to Disease Progression in Meniere Patients. Otol Neurotol 2016;37(10):1614-9.

25. Ochi K, Ohashi T, Nishino H. Variance of vestibular-evoked myogenic potentials. Laryngoscope 2001;111:522-7. 
26. Rahne T, Weiser C, Plontke S. Neurofeedback-controlled comparison of the head elevation versus head rotation and head-hand methods in eliciting cervical vestibular evoked myogenic potentials. Audiol Neurootol 2014;19:327-35.

27. Welgampola MS, Myrie OA, Minor LB, et al. Vestibular-evoked myogenic potential thresholds normalize on plugging superior canal dehiscence. Neurology 2008;70(6):464-72.

28. Niesten ME, McKenna MJ, Herrmann BS, et al. Utility of cVEMPs in bilateral superior canal dehiscence syndrome. Laryngoscope 2013;123(1):226-32. 



\section{Chapter}

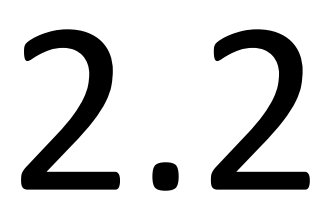

Normalization reduces the need for strong muscle contraction

JJ Guinan Jr. 


\section{Abstract}

\section{Background}

The cervical vestibular evoked myogenic potential (cVEMP) represents an inhibitory reflex of the saccule measured in the ipsilateral sternocleidomastoid muscle (SCM) in response to acoustic or vibrational stimulation. Since the CVEMP is a modulation of SCM electromyographic (EMG) activity, cVEMP amplitude is proportional to muscle EMG amplitude. We sought to evaluate muscle contraction influences on cVEMP peakto-peak amplitude (VEMPpp), normalized cVEMP amplitude (VEMPn) and inhibition depth (VEMPid).

\section{Methods}

cVEMPs at $500 \mathrm{~Hz}$ were measured in twenty-five healthy subjects for three SCM EMG contraction ranges: $45-65,65-105$, and $105-500 \mu \mathrm{V}$ root mean square (rms). For each range, we measured cVEMP sound-level functions (93 to $123 \mathrm{~dB}$ peSPL) and sound off, meaning that muscle contraction was measured without acoustic stimulation. The effect of muscle contraction amplitude on VEMPpp, VEMPn and VEMPid and the ability to distinguish cVEMP presence/absence was evaluated.

\section{Results}

VEMPpp amplitudes were significantly greater at higher muscle contractions. In contrast, VEMPn and VEMPid were not significantly affected by muscle contraction. Cohen's $d$ indicated that for all three CVEMP metrics contraction amplitude variations produced little change in the ability to distinguish cVEMP presence vs. absence. VEMPid more clearly indicated saccular output because when no acoustic stimulus was presented the saccular inhibition estimated by VEMPid was zero, unlike VEMPpp and VEMPn.

\section{Conclusion}

Muscle contraction amplitude strongly affects VEMPpp amplitude, but contractions 45-300 $\mu \mathrm{V}$ rms produce stable VEMPn and VEMPid values. Clinically, there may be no need for subjects to exert high contraction effort. This is especially beneficial in patients for whom maintaining high SCM contraction amplitudes is challenging. 


\section{Introduction}

Cervical vestibular evoked myogenic potentials (cVEMPs) are responses to acoustic or mechanical stimuli measured with electromyography (EMG) on the sternocleidomastoid muscle (SCM). The ipsilateral inhibition produced by this vestibulocollic reflex is measured with surface electrodes on the tonically-contracted SCM (1). This SCM response is predominantly of saccular origin and provides a measure of the integrity of the saccule and inferior vestibular nerve (2). Typically, a CVEMP consists of two peaks: a positive peak and a negative peak, referred to as P1 and N1, occurring approximately 13 and 23 ms after sound onset for clicks (1).

Several metrics can be used to analyze cVEMPs. A common metric is the peak-to-peak amplitude difference between P1 and N1 (VEMPpp). VEMPpp increases with increased muscle contraction (1,3-11). To make VEMPpp comparisons across subjects, contraction amplitude should be kept the same across subjects. Alternately, one can correct for differences in contraction amplitude by dividing VEMPpp by EMG amplitude, which yields the normalized VEMPpp (VEMPn) $(7,9,11,12)$. A third metric, VEMP inhibition depth (VEMPid), estimates the "inhibition depth," defined as the fractional inhibition of SCM motoneuron response produced by activation of the saccule (13). VEMPid uses a template correlation method that resembles a matched filter for the detection of a signal (the CVEMP) in noise. VEMPid provides normalization and improved sensitivity at low response levels (13).

Our focus in this study is to determine the muscle contraction amplitude range over which normalization works well. Normalization produces a constant VEMPn value when VEMPpp grows linearly with contraction amplitude as measured by the muscle EMG amplitude. Many studies have reported that for most subjects VEMPpp increases linearly with contraction amplitude $(1,3,4,7,8,10)$. However, a few studies reported that for some subjects VEMPpp saturates for strong muscle contractions $(5,9,11)$. To achieve a wide range of contraction amplitudes, several different muscle maneuvers (e.g. lifting vs. turning the head) have been used $(5,6,11,14)$. However, with different maneuvers different muscles are activated, which may change the pattern of activation within the SCM. Furthermore, the orientation of the saccule relative to gravity changes with different maneuvers. For instance, Rosengren (11) found VEMPpp vs. EMG nonlinearity for low-amplitude contractions (averaged-full-wave-rectified (RECT) EMGs less than $50 \mu \mathrm{V}$ ) and excluded these from further analysis. However, this nonlinearity may be caused by the use of different head-position maneuvers to produce low-amplitude EMGs. Another factor is the outcome measure considered when choosing a suitable EMG contraction range. Studies optimized for measuring cVEMP interaural asymmetry ratios (IARs) may lead to recommending stronger muscle contractions (11). However, some patients with nominally unilateral Menière's disease (MD) show abnormal cVEMPs in their "unaffected" ear, which indicates that IAR is a flawed VEMP metric 
(15). Here we do not consider IAR and only consider optimizing individual normalized cVEMP measurements.

When obtaining cVEMPs in clinic, a single means of achieving SCM contraction is used and the question is: What is the range of SCM contraction amplitudes that provides consistent cVEMP outcomes? For cVEMP measurements, patients in our clinic are now encouraged to produce contractions that yield root-mean-square (rms) EMGs $>65 \mu \mathrm{V}$ ( $50 \mu \mathrm{V}$ RECT) by sitting up straight and turning their head away from the stimulated ear. Many, especially older, patients find it challenging to reach this contraction amplitude. To avoid requiring patients to make contractions that are unnecessarily strong, and to reduce discomfort, it is important to know how much contraction amplitude influences CVEMP metrics, especially for low-amplitude contractions.

We collected CVEMP data from healthy subjects using one head-turning maneuver to achieve specified low-amplitude muscle-contraction ranges. Our primary aims were: 1) To determine how much muscle contraction amplitude influences cVEMP metrics, 2) to evaluate across sound intensities how well VEMPn and VEMPid correct for differences in contraction amplitudes, and 3) to determine the preferred target contraction range. We found that contractions with 45-300 $\mu \mathrm{V}$ rms EMGs yielded relatively uniform VEMPn and VEMPid values.

\section{Methods}

\section{Subjects}

Twenty-five healthy subjects were included: 9 male and 16 female (mean age: 42.8 years, age range: $22-76$ years). All subjects received air and bone-conduction audiograms at 250, 500, 750, 1000, 2000 and $4000 \mathrm{~Hz}$. Subjects were excluded if they had an air-bone gap $>10 \mathrm{~dB}$ or a history of balance issues, hearing loss or neurologic disease. Informed consent was obtained and signed by all subjects. This study was approved by the Human Studies Committee of the Massachusetts Eye and Ear Infirmary (\#13-097H, PI: S.D. Rauch).

\section{cVEMP testing}

To contract the SCM, subjects sat up straight and turned their head away from the stimulated ear. EMG activity was recorded from four surface electrodes: a positive electrode on the middle belly of each SCM, a reference electrode at the midline point between the SCM attachments to the sternum, and a ground electrode on the midline forehead. Ipsilateral SCM EMG was monitored while subjects maintained rms EMGs within one of three ranges $(45-65,65-105$ and $105-500 \mu \mathrm{V} \mathrm{rms})$ as requested by the experimenter. In our data, rms EMG values averaged 1.32 (1.27-1.45 across subjects) 
times higher than full-wave rectified EMG values, thus the range divisions are: 34.1-49.2, 49.2-79.5 and 79.5 $\mu \mathrm{V}-378.8$ RECT. Before the first test, subjects briefly practiced maintaining SCM contractions within the three ranges. During both practice and tests, subjects received verbal feedback on their muscle contraction amplitude. EMG activity was amplified, bandpass filtered and sampled at $50 \mathrm{kHz}$ with a 16-bit analog-to-digital converter (National Instruments) and all responses were stored.

$500 \mathrm{~Hz}$ tone bursts were generated by custom-programmed evoked potential software (National Instruments 16-bit digital I/O board) using a Blackman gating function with two cycle $(4.0 \mathrm{~ms})$ rise and fall times and no plateau. Tone bursts were presented monaurally with circumaural headphones (Telephonics TDH-49) at a repetition rate of $13 / \mathrm{s}$. Tone bursts were presented at $93,103,113$ and $123 \mathrm{~dB}$ peak-equivalent sound pressure level (peSPL; $93 \mathrm{~dB}$ peSPL is $60 \mathrm{~dB} \mathrm{nHL}$ ). cVEMP responses were recorded for all three contraction ranges for each side and for each sound level. In addition, EMG recordings were obtained without any sound stimulus for all three muscle contraction ranges. For this, subjects contracted their SCM as they did when acoustically stimulated, but no sound was delivered. We randomized presentation order for sound level, side and muscle contraction range. For each recording 200 to 300 single EMG responses were obtained and stored.

\section{Analyses}

VEMPpp was calculated by measuring the amplitude difference between P1 and N1 in the averaged CVEMP waveform. P1 was the largest positive peak in the interval 9 to $20 \mathrm{~ms}$ and $\mathrm{N} 1$ was the largest negative peak after P1 and before $30 \mathrm{~ms}$. Time was measured relative to the peak of the sound stimulus, which gives uniform average delays across different sound frequencies $(5,12)$. These acceptance windows take into account the normal variation in CVEMP latencies across subjects.

VEMPn was calculated from the peak-to-peak amplitude of the averaged, normalized cVEMP waveform. To normalize waveforms, each raw EMG trace was divided by the overall, $77 \mathrm{~ms}$ long, rms amplitude of the same trace (this is termed trace-by-trace normalization) (12). VEMPn was the peak-to-peak value measured from this normalized cVEMP waveform as described for VEMPpp.

To compute VEMPid, a template of the expected cVEMP waveform is required, and for this we used a generic template made by averaging peak-aligned cVEMP responses from 6 other normal subjects (16). The point-by-point correlation of each individual trace with the generic template was calculated (termed the template correlation value or TCV). VEMPid is the mean of all the 200 to 300 TCVs divided by the standard deviation of the TCVs and multiplied by 0.2. A more detailed description of the VEMPid calculation (using a subject-specific template) can be found in Prakash et al. (13). To compensate for the generic template's lack of latency information, TCVs were calculated with the generic template's delay varied to cover the interval 9 to $20 \mathrm{~ms}$ in 
$0.1 \mathrm{~ms}$ steps, and the largest value was used for the VEMPid. The latency that yielded the largest VEMPid at the highest sound level was used for calculating VEMPids at lower sound levels.

Patients were divided in three age groups: 1) 22-29, 2) 30-49 and 3) 50-76 years old, $\mathrm{n}=6,9$ and 10 respectively. For the VEMPpp, VEMPn and VEMPid measurements, we calculated means and standard errors across subjects in every age group at each sound level, side, and muscle contraction range. Full factorial analyses of variance (ANOVAs) were performed to determine the effect of muscle contraction amplitude on each of these metrics. Age, side, sound level and muscle contraction amplitude were considered fixed factors and the subject was considered a variable factor. Data from both ears were pooled for the figures and subsequent analysis.

To examine the degree to which VEMPpp, VEMPn and VEMPid were influenced by muscle contraction, we calculated the eta-squared effect size $\left(\eta^{2}\right)$ for each metric and sound level. Eta-squared effect size is a ratio of variances and gives the proportion of the variance in the cVEMP metric that can be attributed to (in this case) changes in muscle contraction amplitude.

$$
\eta 2=\frac{S S \text { group }}{S S \text { total }}
$$

where: SS is the sum of squares; SSgroup is the sum of the squared differences between the mean $(\mu)$ of every muscle tension group ( $\mu$ group) and the total mean ( $\mu$ total), multiplied by the number in the group, ngroup, i.e.

$$
\text { SSgroup }=\Sigma_{1}^{3} \text { ngroup } \cdot(\mu g r o u p-\mu \text { total })^{2}
$$

and SStotal is the sum of the squared differences between every individual point and the total mean, i.e.

$$
\text { SStotal }=\sum_{i=1}^{n t o t a l}(\chi i-\mu \text { total })^{2}
$$

Eta-squared effect sizes of $0.01,0.06$ and 0.14 are considered small, medium and large, respectively (17).

To determine how well each cVEMP metric was able to distinguish cVEMP presence or absence, Cohen's $d$ effect sizes were calculated using the cVEMP metrics from soundon versus no-sound EMG measurements. Cohen's $d$ is the difference between each group mean $\left(\mu_{1}\right)$ and the corresponding no-sound mean $\left(\mu_{2}\right)$, divided by the combined standard deviation of the two groups $\left(\sigma_{1,2}\right)$.

$$
\text { Cohen's } d=\frac{\mu_{1}-\mu_{2}}{\sigma_{1,2}}
$$


As stated earlier: for each recording 200 to 300 single EMG responses were obtained and used to calculate VEMPpp, VEMPn, VEMPid, eta-squared effect size and Cohen's $d$. Statistical analyses were performed using Matlab (version R2014a) and SPSS (version 22.0; Chicago, IL, USA). A $p$ value of $<0.05$ was considered statistically significant. All post-hoc pairwise comparisons were performed using a Bonferroni adjustment for multiple comparisons.

\section{Results}

Typical cVEMP waveforms from the three muscle-contraction ranges and across sound levels are shown in Figure 2.2.1. Consistent with most previous work, VEMPpp increased almost linearly with increases in muscle contraction amplitude for the three muscle contraction groups regardless of age group (Figure 2.2.2A-C). On average, the increases in VEMPpp with increases in muscle contraction amplitude were significantly greater at higher sound levels as shown by a significant interaction between muscle contraction amplitude and sound level (ANOVA: $\mathrm{F}=15.410, p<0.001$ ). Because of this significant interaction, the effect of muscle contraction amplitude on VEMPpp was calculated separately for each sound level. For all sound levels VEMPpp was significantly higher at higher muscle contraction amplitudes with age groups combined (Table 2.2.1).

Muscle Contraction, EMG Range:

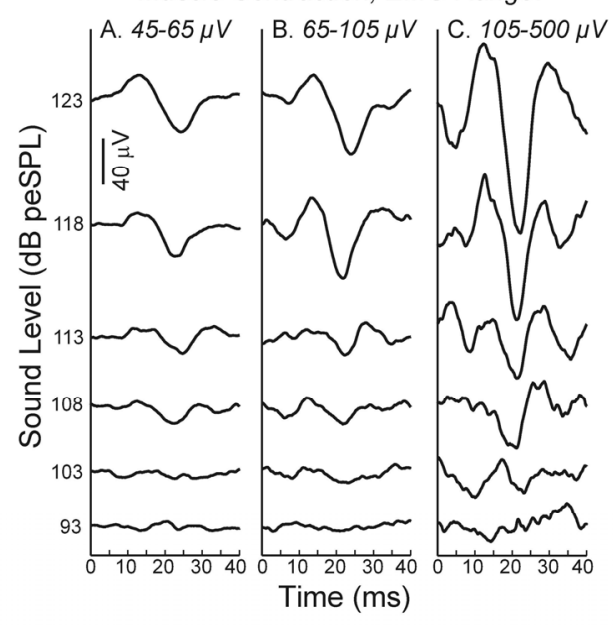

Figure 2.2.1 Example cVEMP traces from one subject showing responses to different stimulus levels while contracting the SCM within the three different muscle contraction amplitude ranges (A-C). Although the cVEMP amplitudes increase with contraction amplitude, the resulting larger cVEMP amplitudes do not improve the ability to determine if a cVEMP is present or not because of the higher level residual noise. All cVEMP displayed in this figure are averages of 200-300 single EMG responses. 

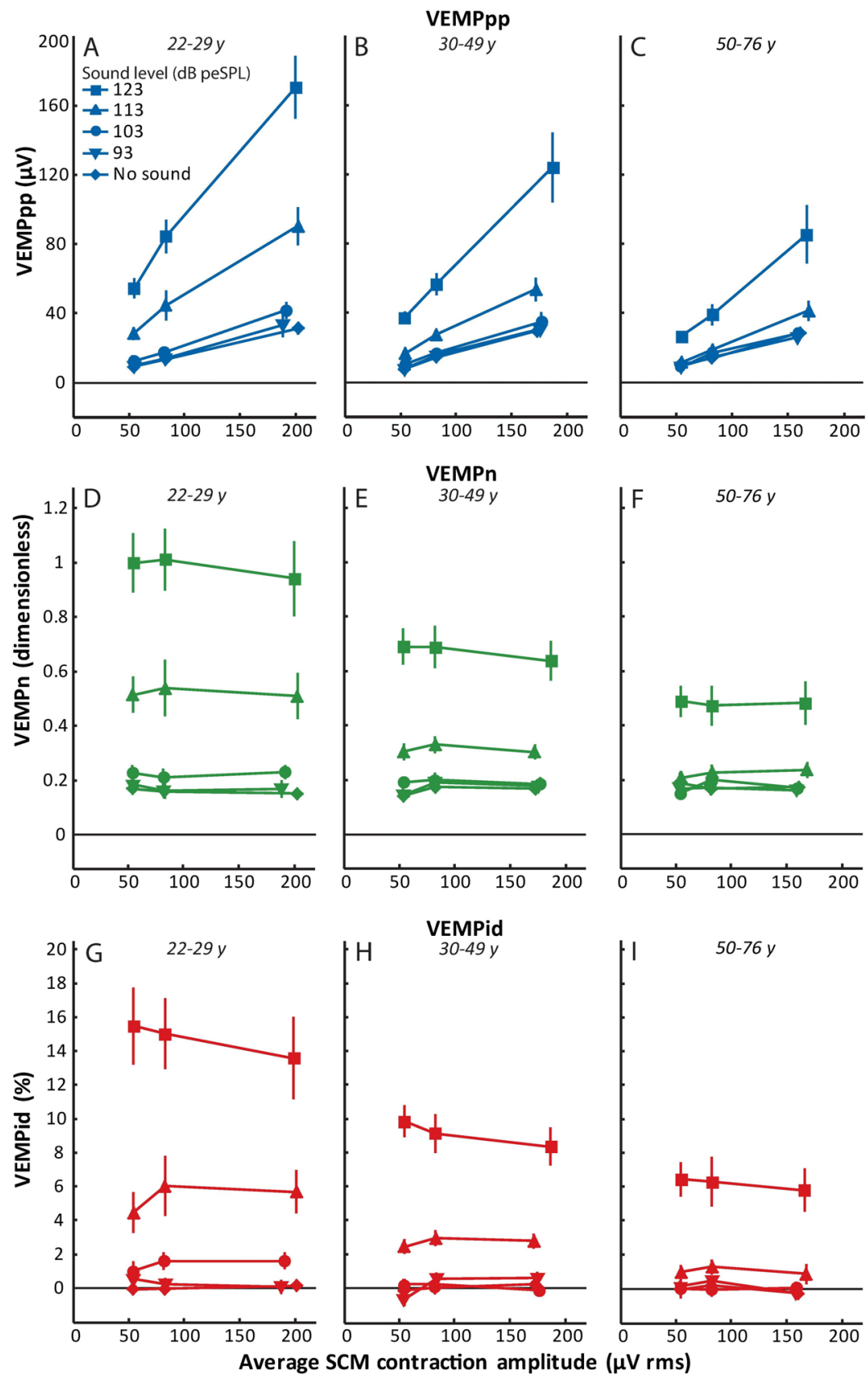

Figure 2.2.2 Average VEMP peak-to-peak (VEMPpp) (A-C), normalized VEMP (VEMPn) (D-F) and VEMP inhibition depth (VEMPid) (G-I) for the three age groups as functions of average muscle contraction amplitude, showing that VEMPpp grows with muscle contraction in all age groups 
(A-C), whereas VEMPn and VEMPid remain stable for muscle contraction amplitudes $>45 \mu \mathrm{V}$ rms in all age groups (D-I). The symbols represent data from stimulus levels 93, 103, 113, 123 dB peSPL and sound off within the predefined muscle contraction ranges (45-65, 65-105 and 105-500 $\mu \mathrm{V} \mathrm{rms)} \mathrm{from} \mathrm{all} \mathrm{subjects} \mathrm{(50} \mathrm{ears).} \mathrm{Error} \mathrm{bars} \mathrm{indicate} \mathrm{the} \mathrm{standard} \mathrm{error} \mathrm{of} \mathrm{the} \mathrm{mean.}$

\begin{tabular}{ccccccc}
\hline & \multicolumn{2}{c}{ VEMPpp } & \multicolumn{2}{c}{ VEMPn } & \multicolumn{2}{c}{ VEMPid } \\
\hline Sound level (dB peSPL) & F value & $p$ value & F value & $p$ value & Fvalue & $p$ value \\
93 & 8.727 & $<0.001^{*}$ & 0.035 & 0.966 & 0.225 & 0.798 \\
103 & 11.156 & $<0.001^{*}$ & 0.103 & 0.902 & 0.015 & 0.985 \\
113 & 32.005 & $<0.001^{*}$ & 0.157 & 0.885 & 0.488 & 0.614 \\
123 & 132.353 & $<0.001^{*}$ & 0.466 & 0.628 & 1.684 & 0.187 \\
\hline
\end{tabular}

Table 2.2.1 The effect of muscle contraction on VEMPpp, VEMPn and VEMPid (F values and $p$ values) for every sound level separately. VEMPpp is significantly affected by muscle contraction amplitude for all sound levels, whereas VEMPn and VEMPid are not significantly affected by muscle contraction amplitude at any sound level.

Average P1 latencies decreased from $14.0 \mathrm{~ms}$ to $13.0 \mathrm{~ms}$ for the $45-65 \mu \mathrm{V}$ and $65-105 \mu \mathrm{V}$ groups respectively (Bonferroni adjusted $p=0.012$ ), and there was no statistically significant change comparing $65-105 \mu \mathrm{V}$ and $105-500 \mu \mathrm{V}$ (13.0 to $13.5 \mathrm{~ms}$; Bonferroni adjusted $p=0.559$ ). Average N1 latencies were not statistically significantly different across muscle contraction amplitude groups (from lowest to highest contraction group: $23.2,22.8$ and $22.7 \mathrm{~ms} ; p=0.375$ ).

In contrast to VEMPpp, both VEMPn and VEMPid showed no significant effect of the three muscle contraction amplitudes, controlling for possible interactions with sound level and side (ANOVA: $F=0.338, p=0.713$ and $F=0.605, p=0.546$, respectively; Figure 2.2.2D-I). Both VEMPn and VEMPid increased as sound level was increased and there was an interaction between age and sound level $(F=15.060, p<0.001$; $F=12.797, p<0.001$ ). Therefore, the effect of sound level on VEMPn and VEMPid was calculated separately for each age group. The effect of sound level was significant in all age groups for both VEMPn and VEMPid (all age groups: $p<0.001$ ) and the effect of age was only significant at the highest sound levels (VEMPn at 93, 103, 113 and $123 \mathrm{~dB}$ peSPL: $\mathrm{F}=0.005, p=0.995 ; \mathrm{F}=0.650, p=0.522 ; \mathrm{F}=25.213, p<0.001 ; \mathrm{F}=71.104, p<$ 0.001. VEMPid at 93, 103, 113 and $123 \mathrm{~dB}$ peSPL: $\mathrm{F}=0.038, p=0.962 ; \mathrm{F}=2.117, p=$ $0.121 ; \mathrm{F}=18.085, p<0.001 ; \mathrm{F}=68.434, p<0.001)$. In contrast to VEMPpp, the effect of muscle contraction amplitude on VEMPn and on VEMPid was not significant when each sound level was considered individually (Table 2.2.1).

To get a quantitative measure of how much muscle contraction amplitude affected the various CVEMP metrics at each sound level, we calculated the $\eta^{2}$ effect size across the three contraction ranges (Figure 2.2.3). Because the effects of muscle contraction amplitude are similar amongst the different age groups, data of all age groups were combined to calculate the $\eta^{2}$ effect size. According to the standard assessments for $\eta^{2}$ (see Methods), muscle contraction amplitude had a very large effect on VEMPpp $\left(\eta^{2}=0.32-0.45\right)$, a small effect on VEMPn $\left(\eta^{2}<0.01\right)$ and a small effect on VEMPid $\left(\eta^{2}=0.01-0.02\right)$ (Figure 2.2.3). 


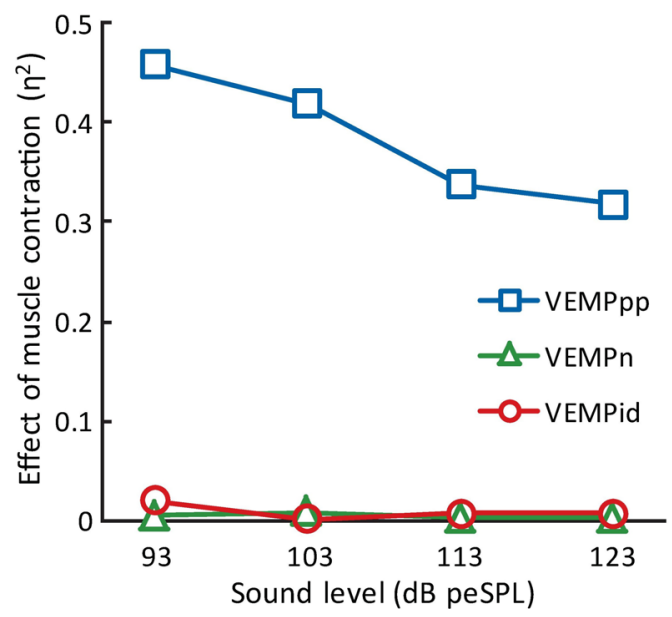

Figure 2.2.3 Eta-squared effect size $\left(\eta^{2}\right)$ assessment of the influence of muscle contraction amplitude on VEMPpp, VEMPn and VEMPid. Effect sizes of $0.01,0.06$ and 0.14 are considered small, medium and large, respectively. Muscle contraction had a large effect on VEMPpp $\left(\eta^{2}=0.32-0.45\right)$, a small effect on VEMPn $\left(\eta^{2}<0.01\right)$ and a small effect on VEMPid $(0.01-0.02)$.

Since VEMPn and VEMPid did not change significantly across muscle contractions for EMGs down to at least $45 \mu \mathrm{V}$ rms (Figure 2.2.2D-I), we explored whether even lower contractions might be acceptable. Most subjects maintained their contraction amplitudes within the predefined ranges, but some occasionally produced contractions below the minimum targeted contraction of $45 \mu \mathrm{V}$ rms. We used these data when there were 100 or more responses within the $25-45 \mu \mathrm{V}$ rms muscle contraction range (At 93, 103, 113 and $123 \mathrm{~dB}$ peSPL in age group $1 \mathrm{n}=7,6,5,5$ ears; in age group 2 $n=13,11,11,13$ ears; in age group $3 n=13,13,9,11$ ears, respectively). No subject had 100 or more responses available within the $0-25 \mu \mathrm{V}$ rms contraction range. In the 25-45 $\mu \mathrm{V}$ rms range, VEMPpp continued its near-linear relationship with muscle contraction amplitude in all age groups, while VEMPn and VEMPid values in the 25-45 $\mu \mathrm{V}$ rms range decreased somewhat (data not shown). None of the VEMPn or VEMPid changes in the $25-45 \mu \mathrm{V}$ rms range (relative to the $45-65 \mu \mathrm{V}$ rms range) were statistically significant.

The data of Figure 2.2.2 are illustrated in Figure 2.2.4 as functions of sound level. This figure shows that VEMPid values are near zero when no stimulus was presented. In contrast, VEMPpp and VEMPn values reached a floor above zero that is due to the baseline EMG variation. This figure shows that VEMPid better represents the saccular output than VEMPpp and VEMPn in that when no stimulus is presented the estimated inhibition depth is zero. 

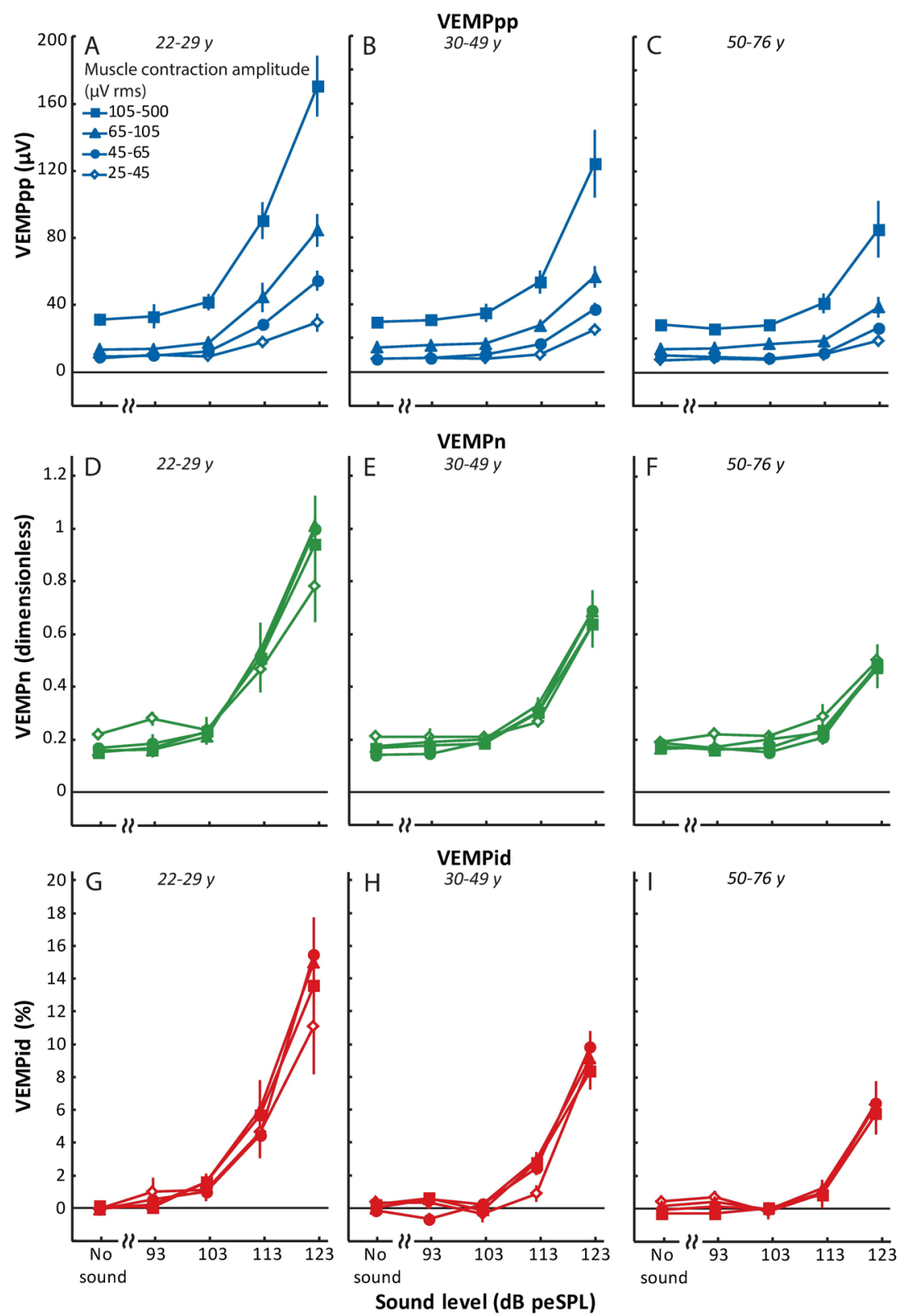

Figure 2.2.4 Average VEMP peak-to-peak (VEMPpp) (A-C), normalized VEMP (VEMPn) (D-F) and VEMP inhibition depth (VEMPid) (G-I) for all age groups as functions of sound level (no sound, 93, 103, 113 and 123 peSPL), showing that in all age groups VEMPpp, VEMPn and VEMPid all grow with sound level while VEMPid is the only metric that extends down to zero when no sound is presented, suggesting that VEMPid is the most accurate measure of true saccular function. Error bars indicate the standard error of the mean. 
To measure how well sound-evoked cVEMPs can be distinguished from no-sound EMG measurements, we used the Cohen's $d$ effect size. Cohen's $d$ indicated that contraction amplitude produced little change in the ability to distinguish cVEMP presence versus absence for all three cVEMP metrics (Figure 2.2.5). The Cohen's $d$ decreased with age, which can be expected given that older patients have lower outcomes for VEMPpp, VEMPn and VEMPid (Figure 2.2.2). This indicates that threshold increases with age, which has been shown before (18-20). For all the cVEMP metrics, Cohen's $d$ increased with sound level but the differences in Cohen's $d$ across contraction ranges were small for all age groups.

For the no-sound recordings, we performed the same CVEMP computations as were done for sound-on recordings. The values obtained from no-sound recordings are not true cVEMP measurements; instead they show a noise-floor for these metrics. The nosound recordings showed the same trends as the sound-on recordings: VEMPpp increased with muscle contraction amplitude (Figure 2.2.2A-C, diamonds) whereas VEMPn and VEMPid were little influenced by muscle contraction amplitude (Figure 2.2.2D-I, diamonds). This is what is expected considering that as muscle contraction amplitude goes up, rms EMG goes up and the maximum positive and negative excursions of the EMG go up, which makes VEMPpp go up. In contrast, both VEMPn and VEMPid provide normalization mechanisms that remove the increase due to increasing EMG, so they are little affected by muscle contraction amplitude.

\section{Discussion}

This study evaluates the effect of muscle contraction amplitude in 25 healthy subjects (50 ears) on three cVEMP metrics: raw peak-to-peak amplitude (VEMPpp), normalized peak-to-peak amplitude (VEMPn) and VEMP inhibition depth (VEMPid). Our data show that muscle contraction amplitude strongly affects VEMPpp values, but has little effect on VEMPn or VEMPid for 45 to $\sim 300 \mu \mathrm{V}$ rms contraction amplitudes across all sound levels. Although all three CVEMP metrics decreased with age, muscle contraction amplitude had little effect on VEMPn and VEMPid in all age groups (Figure 2.2.2). While VEMPn and VEMPid did an equally good job at reducing the effects of contraction amplitude, VEMPid is more representative of saccular output because it provides a "meaningful zero": with no stimulus, the estimated saccular inhibition is zero (Figure 2.2.4G-I).

Although we did not ask subjects to target contraction amplitudes below $45 \mu \mathrm{V}$ rms, there are adequate data to show that at most above-threshold sound levels, VEMPn and VEMPid values averaged slightly lower in the $25-45 \mu \mathrm{V}$ rms range than in the 45-65 $\mu \mathrm{V}$ rms range. This is consistent with findings by Rosengren who also found lower normalized peak-to-peak amplitudes at very low contraction levels (11). Whether contractions below $45 \mu \mathrm{V}$ rms should be used clinically is uncertain given the reduced 
amount of available data below $45 \mu \mathrm{V}$ rms. For even lower contraction amplitudes, $<25 \mu \mathrm{V}$ rms, we have no data and cannot say whether such low contraction amplitudes might be usable. Until the use of very low contraction amplitudes is studied more thoroughly we do not recommend using contraction amplitudes $<45 \mu \mathrm{V}$ rms.

We did not explore the upper limit of muscle contraction amplitude and therefore did not determine what upper limit (if any) should be set on muscle contraction amplitudes. Several studies reported that VEMPpp sometimes saturated at very high contraction amplitudes $(1,9,11)$, with the lowest EMG at which saturation was found being at EMG levels $224 \mu \mathrm{V}$ RECT (1). In one outlying study, Bogle et al. (5) found apparent VEMPpp "saturation" starting near $25 \mu \mathrm{V}$ RECT and did not find any EMG range over which VEMPn was constant. The origin of the aberrant findings of Bogle et al. (5) is unclear, but might be from the use of several different head maneuvers to obtain a wide range of contraction-amplitudes. The more common finding of nonlinearity only for EMGs $\geq 225 \mu \mathrm{V}$ RECT indicates that very high contraction amplitudes should be avoided. Many patients have trouble contracting their SCM over $65 \mu \mathrm{V}$ rms ( $~ 50 \mu \mathrm{V}$ RECT) and will never reach amplitudes over $100 \mu \mathrm{V}$ rms with the method of SCM activation used in this study: sitting up straight and turning the head away from the stimulated ear. Nonetheless, some patients can produce high EMG amplitudes. To avoid VEMPpp saturation they should not be encouraged to contract at extremely high amplitudes.

Prior studies have suggested target muscle contraction amplitudes and explored how well normalization removes the effects of contraction amplitude. Early studies, before normalization became an established technique, considered that to compare VEMPpp measurements across subjects, muscle EMG amplitudes had to be kept constant. Akin et al. (4) proposed a target level of 30-50 $\mu \mathrm{V}$ rms. They did not use normalization, therefore it is unclear whether their data from contractions of 30-50 $\mu \mathrm{V}$ rms produced the same normalized peak-to-peak amplitude as their data from higher contraction amplitudes. Davenport (6) proposed a target EMG level of $50 \mu \mathrm{V}$ RECT. McCaslin et al. (9) looked at how well normalization stabilized the peak-to-peak amplitudes using a design somewhat similar to ours but with high target levels $(100,200,300,400 \mu \mathrm{V}$ RECT) and found normalization produced uniform peak-to-peak amplitudes for contractions 100-300 $\mu \mathrm{V}$ RECT but not for $400 \mu \mathrm{V}$ RECT. Rosengren et al. (21) recommended 40 to $\sim 150-200 \mu \mathrm{V}$ RECT be used. To minimize the chance of getting high IAR values, in a later study Rosengren (11) recommended a minimum EMG of $100 \mu \mathrm{V}$ $\mathrm{RECT}$, but also suggested that $80 \mu \mathrm{V}$ RECT might be a more reasonable clinical target. Finally, the proposed guidelines for the clinical application of cervical vestibular evoked myogenic potentials (22) state that: "it is desirable that average rectified or RMS muscle activity is kept between 50 and $200 \mu \mathrm{V}$." Here, we show that for contraction amplitudes of 45-300 $\mu \mathrm{V}$ rms (equivalent to 34-227 $\mu \mathrm{V}$ RECT), uniform values are produced by normalization of VEMPpp or by using VEMPid. Thus, any contraction within this range should be equivalently useful. 
Above we considered the effect of muscle contraction amplitude on the values of cVEMP metrics. However, these values do not fully convey the ability to detect the presence vs. absence of a cVEMP response. This ability was evaluated using Cohen's $d$ which indicated that muscle contraction amplitude has little or no effect on the ability to detect cVEMP presence versus absence (Figure 2.2.5). It is notable that VEMPpp does just as well as VEMPn and VEMPid for detecting CVEMP presence vs. absence. These similar detection abilities can be understood by considering that when visualizing a recording to determine whether a cVEMP is present or not, the waveshape is more important than the amplitude, and normalization changes the amplitude but has little effect on the waveshape. Also, as contraction amplitude goes up, so does the background noise (Figure 2.2.1).

The strengths of our study are that all subjects had to maintain the same predefined muscle contraction amplitudes using a single maneuver - turning the head with different amounts of effort. In addition, muscle contraction was continuously monitored and contractions outside the targeted range were excluded from analyses of that target-range group. Furthermore we tested our subjects at muscle contraction amplitudes that can realistically be reached by patients with the method of SCM activation used in this study, so that our results will be clinically relevant. In contrast to most other studies, all of our subjects were measured over a range of sound levels, including a "no sound" control, while evaluating different targeted contraction strengths. This allowed for the analysis of the effects of different contraction amplitudes on different cVEMP metrics at both high and low sound levels. The results showed that normalization works at both low and high sound levels and across age groups. Our results can also be expected to apply to patients, as long as the patient population does not have a muscle-control problem. However, this remains to be demonstrated experimentally.

\section{Conclusion}

In summary, although muscle contraction amplitude strongly affects VEMPpp amplitude, throughout the range of $45-300 \mu \mathrm{V}$ rms contraction amplitude has little effect on VEMPn or VEMPid, and cVEMP detection is reliable. Clinically, this means there may be no need for subjects to exert maximal or high muscle contraction effort when using VEMPn or VEMPid, which is especially beneficial in patients for whom reaching high levels of SCM contractions can be challenging. 


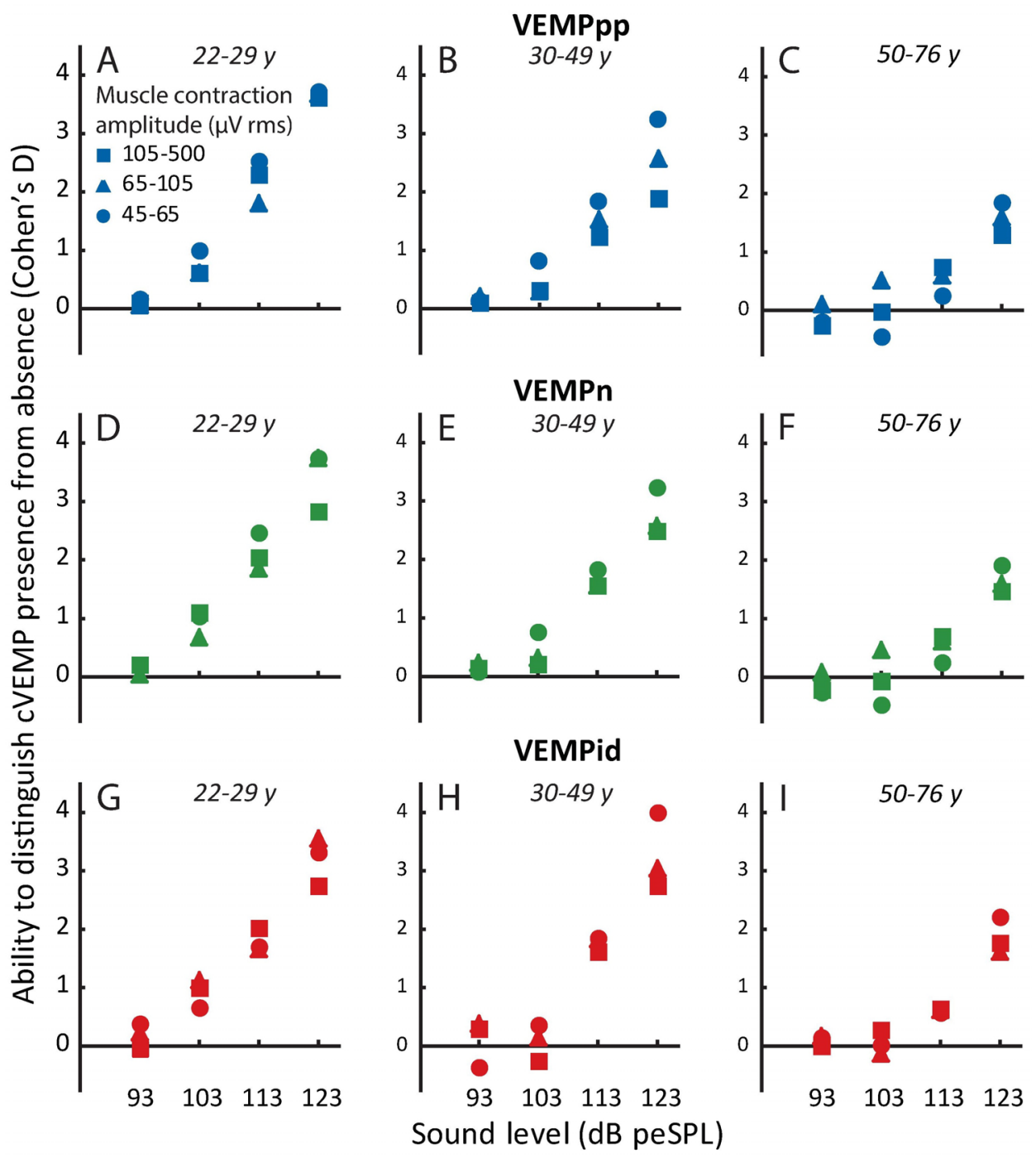

Figure 2.2.5 Cohen's $d$ effect size, which assesses the ability to distinguish cVEMP presence or absence, for VEMPpp (A-C), VEMPn (D-F) and VEMPid (G-I) for all age groups. Cohen's $d$ increases with an increase in sound level but shows no consistent pattern in the effects of muscle-contraction amplitude in any of the age groups, i.e. the contraction amplitudes that produced the highest or lowest Cohen's $d$ vary and seem random. 


\section{References}

1. Colebatch JG, Halmagyi GM, Skuse NF. Myogenic potentials generated by a click-evoked vestibulocollic reflex. J Neurol Neurosurg Psychiatry 1994;57:190-7.

2. Curthoys IS. A critical review of the neurophysiological evidence underlying clinical vestibular testing using sound, vibration and galvanic stimuli. Clin Neurophysiol 2010;121:132-44.

3. Akin FW, Murnane OD. Vestibular evoked myogenic potentials: preliminary report. J Am Acad Audiol 2001;12:445-52.

4. Akin FW, Murnane OD, Panus PC, et al. The influence of voluntary tonic EMG level on the vestibularevoked myogenic potential. J Rehabil Res Dev 2004;41:473-80.

5. Bogle JM, Zapala DA, Criter R, et al. The effect of muscle contraction level on the cervical vestibular evoked myogenic potential (cVEMP): usefulness of amplitude normalization. J Am Acad Audiol 2013;24:77-88.

6. Davenport MJ. The Effect of Sternocleidomastoid Muscle Activation Pattern and Feedback Condition on the Vestibular Evoked Myogenic Potential. Electronic Thesis and Dissertations, East Tenessee State University 2010, Paper 1776.

7. Isaacson B, Murphy E, Cohen H. Does the method of sternocleidomastoid muscle activation affect the vestibular evoked myogenic potential response? J Vestib Res 2006;16:187-91.

8. $\operatorname{Lim} \mathrm{CL}$, Clouston $\mathrm{P}$, Sheean $\mathrm{G}$, et al. The influence of voluntary EMG activity and click intensity on the vestibular click evoked myogenic potential. Muscle Nerve 1995;18:1210-3.

9. McCaslin DL, Fowler A, Jacobson GP. Amplitude normalization reduces cervical vestibular evoked myogenic potential (cVEMP) amplitude asymmetries in normal subjects: proof of concept. J Am Acad Audiol 2014;25:268-77.

10. Ochi K, Ohashi T, Nishino $\mathrm{H}$. Variance of vestibular-evoked myogenic potentials. Laryngoscope 2001;111:522-7.

11. Rosengren SM. Effects of muscle contraction on cervical vestibular evoked myogenic potentials in normal subjects. Clin Neurophysiol 2015;126:2198-206.

12. van Tilburg MJ, Herrmann BS, Guinan JJ Jr, et al. Normalization reduces intersubject variability in cervical vestibular evoked myogenic potentials. Otol Neurotol 2014;35:e222-7

13. Prakash SR, Herrmann BS, Milojcic R, et al. Evaluating Inhibition of Motoneuron Firing From Electromyogram Data to Assess Vestibular Output Using Vestibular Evoked Myogenic Potentials. Ear Hear 2015;36:591-604.

14. Wang $\mathrm{CT}$, Young $\mathrm{YH}$. Comparison of the head elevation versus rotation methods in eliciting vestibular evoked myogenic potentials. Ear Hear 2006;27:376-81.

15. Rauch SD, Zhou G, Kujawa SG, et al. Vestibular evoked myogenic potentials show altered tuning in patients with Ménière's disease. Otol neurotol 2004;25:333-8.

16. Noij KS, Hermann BS, van Tilburg MJ, et al. Toward Optimizing VEMP: Calculating VEMP Inhibition Depth (VEMPid) with a Generic Template. Asso Res Otolaryngol MidWinter Meeting 2017, Abstr 451, p40.

17. Cohen J. The Analysis of Variance and Covariance; in Cohen J: Statistical Power Analysis for the Behavioral Sciences. New York, Lawrence Erlbaum Associates, 1988, pp 283-355.

18. Ochi K, Ohashi T. Age-related changes in the vestibular-evoked myogenic potentials. Otolaryngol Head Neck Surg 2003;129:655-9.

19. Basta D, Todt I, Ernst A. Characterization of age-related changes in vestibular evoked myogenic potentials. J Vestib Res 2007;17:93-8.

20. Singh NK, Kashyap RS, Supreetha L,et al. Characterization of age-related changes in sacculocolic response parameters assessed by cervical vestibular evoked myogenic potentials. Eur Arch Otorhinolaryngol 2014;271:1869-77.

21. Rosengren SM, Welgampola MS, Colebatch JG. Vestibular evoked myogenic potentials: past, present and future. Clin Neurophysiol 2010;121:636-51.

22. Papathanasiou ES, Murofushi T, Akin FW, et al. International guidelines for the clinical application of cervical vestibular evoked myogenic potentials: An expert consensus report. Clin Neurophysiol 2014;125:658-666. 




\section{Chapter}

Clinical use of cVEMP in patients with superior semicircular canal dehiscence syndrome 



\title{
Chapter
}

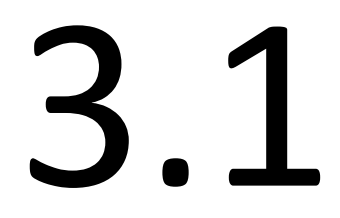

Combining air-bone gap and cVEMP thresholds to improve diagnosis of superior canal dehiscence syndrome

\author{
KS Noij \\ MJ Duarte \\ K Wong \\ YS Cheng \\ S Masud \\ BS Herrmann \\ HD Curtin \\ VV Kanumuri \\ JJ Guinan Jr. \\ ED Kozin \\ O Tarabichi \\ DH Jung \\ DJ Lee \\ SD Rauch
}

Otology \& Neurotology 2018;39(2):212-220 


\section{Abstract}

\section{Objective}

To develop a novel approach combining low frequency air-bone gap ( $A B G$ ) and cervical vestibular evoked myogenic potential (cVEMP) thresholds to improve screening for superior canal dehiscence syndrome (SCD).

\section{Study design}

Retrospective study

Setting: Tertiary care center

\section{Patients}

140 patients with SCD and 21 healthy age-matched controls were included. Ears for each patient were divided into three groups based on computed tomography (CT) findings: 1) dehiscent, 2) thin, or 3) unaffected.

\section{Main outcome measures}

cVEMP and audiometric thresholds were analyzed and differences among groups were evaluated.

\section{Results}

We define the third window indicator (TWI) as the CVEMP thresholds at 500, 750 and $1000 \mathrm{~Hz}$ adjusted for the air-bone gap (ABG) at $250 \mathrm{~Hz}$ (i.e. subtracting $A B G$ from cVEMP threshold). The TWI differentiates between dehiscent and nondehiscent control ears with a sensitivity of $82 \%$ and specificity of $100 \%$, corresponding to a positive predictive value of $100 \%$. ABGs and CVEMP thresholds were similar for healthy controls and patients with thin bone covering the superior semicircular canal.

\section{Conclusion}

This is the largest study to date examining the usefulness of cVEMPs in the diagnosis of SCD. Our "third window indicator" (TWI) combines CVEMP thresholds with the ABG at $250 \mathrm{~Hz}$ to improve the ability to screen patients with SCD symptoms. 


\section{Introduction}

Superior canal dehiscence syndrome (SCD) is characterized by a bony defect of the superior semicircular canal (SSC) that leads to a variety of auditory and vestibular symptoms (1). A bony dehiscence of the SSC creates a pathologic "third window" that shunts energy from stapes footplate motion away from the cochlear partition. This results in higher energy transmission to the vestibular sense organs and a corresponding decrease in pressure difference across the basilar membrane. This third window phenomenon can cause dizziness, Valsalva-induced vertigo and hearing loss $(2,3)$.

SCD can be challenging to diagnose and patients often see a number of providers for evaluation prior to a final diagnosis. Multiple tests are involved in the workup of SCD patients including high resolution temporal bone computed tomography (CT) as well as audiometric and vestibular testing. The workup of SCD can be time consuming and costly. At our institution, the diagnosis of SCD is based on a combination of symptomatology, exam findings, threshold audiometry and immittance testing, cervical vestibular evoked myogenic potential (cVEMP) testing and temporal bone CT imaging. Patients who pursue surgery also undergo high resolution temporal bone magnetic resonance imaging (MRI) to exclude intracranial pathology (4).

cVEMP testing measures saccular and inferior vestibular nerve function and is mostly used to assess patients with SCD and Menière's disease (5-9). The CVEMP might be a valuable screening tool for patients in whom SCD is suspected $(7,10-12)$. The CVEMP reflex pathway begins with acoustic or vibrational inputs that mechanically stimulate the saccule, leading to predominantly ipsilateral inhibition of the sternocleidomastoid muscle (SCM) as measured with electromyography (EMG) (13). Patients with SCD have lower thresholds and larger amplitudes on CVEMP testing compared to non-SCD patients, although there is overlap with the normal population $(8-10,14-19)$.

While the CVEMP is a relatively inexpensive and potentially efficient way to quickly assess SCD status the sensitivity, specificity and positive predictive values are suboptimal for clinical use $(10,11)$. Improving the use of cVEMP would be valuable in streamlining the diagnosis of SCD.

The aim of this study is to improve the usefulness of cVEMPs as a primary screening test for SCD. We hypothesize that a combination of cVEMP thresholds with lowfrequency audiometric air-bone gap (ABG) will improve the differentiation of SCD from non-SCD ears. 


\section{Methods}

\section{Subjects}

We identified patients with audiometric, CVEMP, and CT imaging data from our SCD database (2000-2016). All subjects were evaluated at our institution. Patients with middle ear pathology based on exam findings and/or impedance audiometry were excluded as tympanic membrane or middle ear abnormalities will influence (increase) cVEMP thresholds. Figure 3.1.1 illustrates our decision tree for inclusion in this study. For comparison, a group of age-matched healthy controls with audiometric and CVEMP data was included.

\section{Radiology}

The majority of the temporal bone $\mathrm{CT}$ images were obtained at our institution using either a multidetector row CT scanner (Somatom Sensation 40; Siemens Healthcare, Erlangen, Germany or Discovery CT750 HD; GE Healthcare, Milwaukee, WI. The Siemens scanner was replaced by the GE scanner in 2014) or a cone beam CT scanner (3D Accuitomo 170; J. Morita, Irvine, CA). Multidetector row and cone beam CT scanner generated images with a slice thickness of 0.63 and $0.5 \mathrm{~mm}$, respectively. The axial pixel dimension of the cone beam scans was $0.125 \mathrm{~mm}$. The slice thickness of outside hospital scans in the axial view ranged from 0.5 to $1.25 \mathrm{~mm}$ (mean: $0.67 \mathrm{~mm}$ ). Superior semicircular canal morphology was evaluated on reformatted images in Stenvers and Pöschl planes using multiplanar reconstruction. Stenvers plane images were perpendicular to the plane of the SSC and Pöschl images were parallel to the SSC. All scans were evaluated by a neuroradiologist at our institution (Figure 3.1.2). Based on CT scan interpretation, superior canals were divided into three groups: dehiscent, thin bone or unaffected ("unaffected" meaning a normal bony covered superior canal of the contralateral ear in patients with unilateral SCD). If the report was equivocal a senior neuroradiologist re-evaluated the scan.

\section{Audiometry}

A comprehensive audiogram, including air- and bone-conduction thresholds was obtained for all subjects included in this study $(20,21)$. If the difference between airand unmasked bone-conduction thresholds was larger than $10 \mathrm{~dB}$, bone-conduction thresholds were masked. The air-bone gap ( $A B G)$ was calculated at each tested frequency by subtracting the bone-conduction threshold from the air-conduction threshold. Patients with an $A B G>10 \mathrm{~dB}$ at any of the tested frequencies were further evaluated with immittance audiometry. Ears with either an $A B G$ of $\leq 10 \mathrm{~dB}$ at any frequency or an $A B G>10 \mathrm{~dB}$ with normal tympanograms and present acoustic reflexes 
were considered to have normal middle ear function and were included for analysis of cVEMP thresholds (Figure 3.1.1).
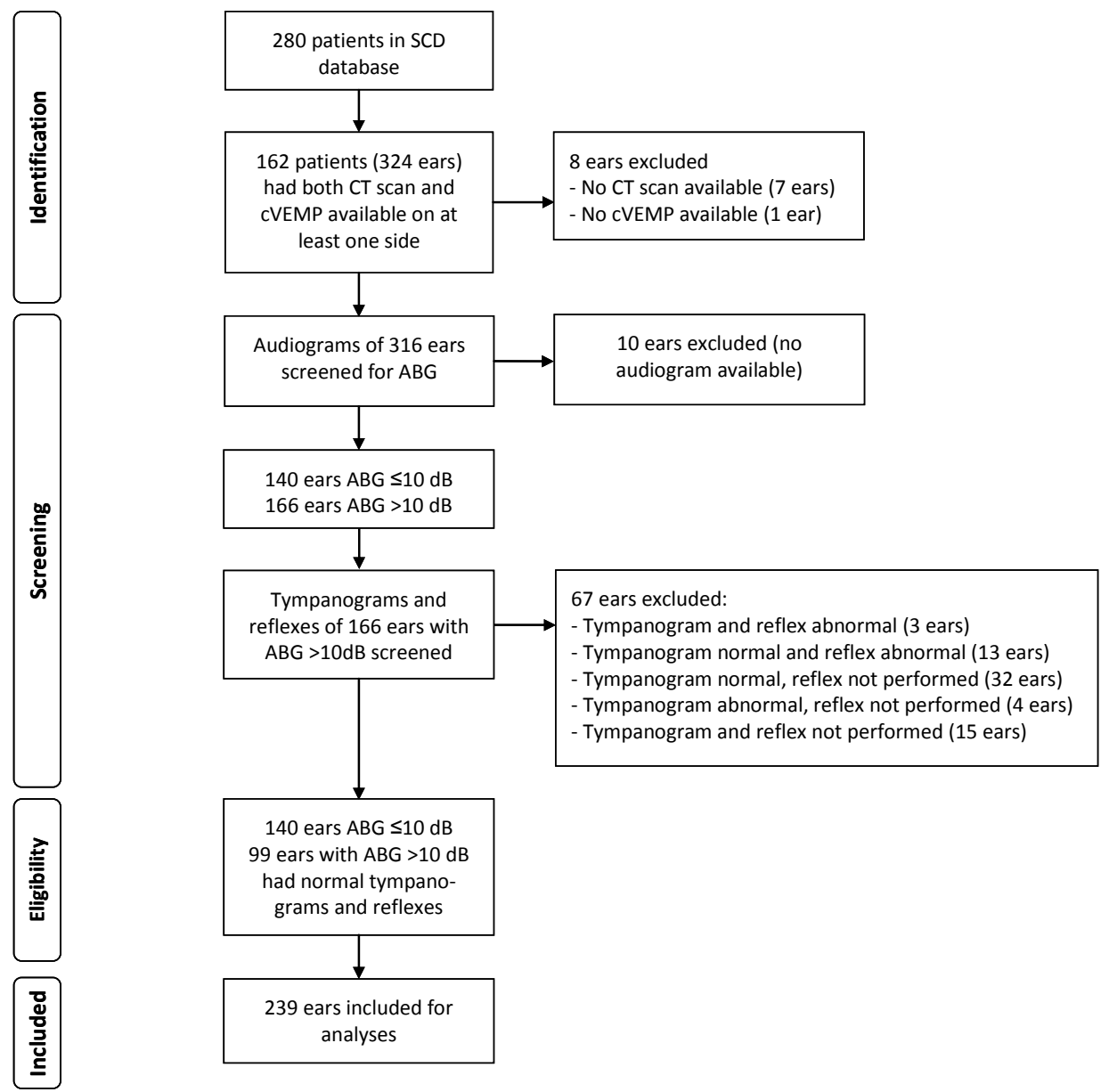

Figure 3.1.1 Flow chart displaying patient inclusion criteria. All 280 patients in the SCD database were screened. All ears with available CT imaging and CVEMP data were screened for middle ear pathology using comprehensive audiometry (threshold audiogram, tympanometry, and acoustic reflex testing). Ears with possible middle ear pathology were excluded from analyses as an increase in middle ear impedance (e.g., from ossicular fixation or otitis media) increases cVEMP threshold values. CT indicates computed tomography; cVEMP, cervical vestibular evoked myogenic potential; SCD, superior canal dehiscence. 


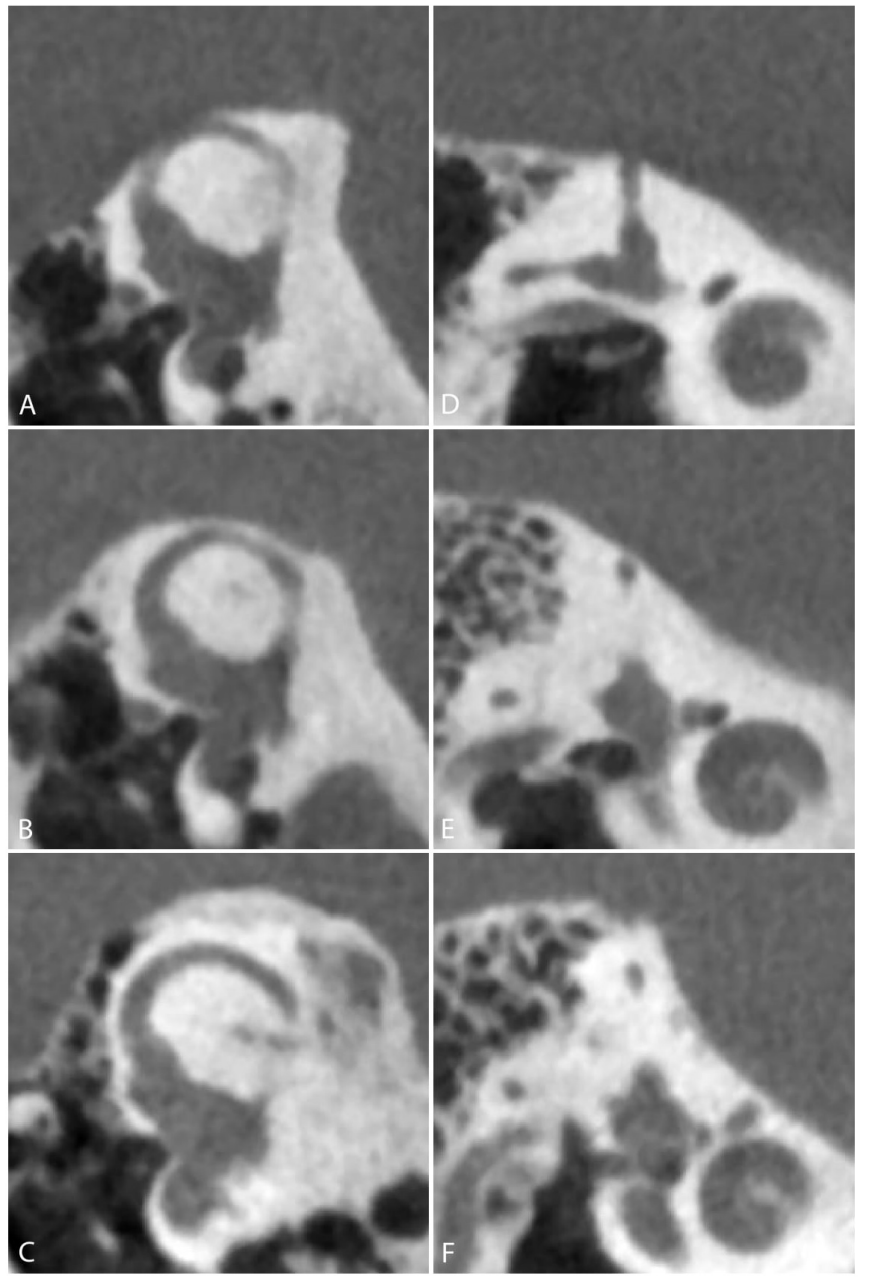

Figure 3.1.2 Cone beam temporal bone CT imaging in Pöschl (A-C) and Stenvers (D-F) planes of three different superior semicircular canals with dehiscence of the arcuate eminence $(A$ and $D)$, thin overlying bone ( $\mathrm{B}$ and $\mathrm{E}$ ) and unaffected bone ( $\mathrm{C}$ and $\mathrm{F}$ ).

\section{CVEMP}

cVEMP thresholds were measured during sternocleidomastoid muscle (SCM) contraction. Subjects were placed in a sitting position with the head turned away from the stimulated ear to elicit contraction of the ipsilateral SCM. Electromyography (EMG) activity was recorded from four surface electrodes: a positive electrode on the middle belly of each SCM, a reference electrode at the midpoint between SCM attachments to the sternum, and a ground electrode on the midline forehead. Ipsilateral SCM EMG 
amplitude was monitored while subjects contracted their SCM $>65 \mu \mathrm{V}$ root mean square (rms). EMG activity was amplified, bandpass filtered and sampled at $50 \mathrm{kHz}$ with a 16-bit analog-to-digital converter (National Instruments).

Next, 500, 750 and $1000 \mathrm{~Hz}$ tone bursts were generated by custom-programmed evoked potential software (National Instruments 16-bit digital $\mathrm{I} / \mathrm{O}$ board) using a Blackman gating function with two cycle $(4.0 \mathrm{~ms}$ at $500 \mathrm{~Hz}, 2.5 \mathrm{~ms}$ at $750 \mathrm{~Hz}$ and $2 \mathrm{~ms}$ at $1000 \mathrm{~Hz}$ ) rise and fall times and no plateau. Tone bursts were presented monaurally with circumaural headphones (Telephonics TDH-49) at a repetition rate of 13 bursts/s. To determine cVEMP thresholds, responses were first obtained at $123 \mathrm{~dB}$ peSPL (peak sound pressure level; $123 \mathrm{~dB}$ peSPL is equivalent to $90 \mathrm{~dB} \mathrm{nHL}$ ) after which the sound level was decreased in $10 \mathrm{~dB}$ steps until no response could be distinguished from residual noise. To determine threshold, sound levels were then raised by $5 \mathrm{~dB}$. Threshold was defined as the lowest sound level at which a CVEMP was present as determined by the audiologist performing the CVEMP. If no response was identified at the highest possible stimulus intensity (133 dB peSPL) CVEMP threshold was defined as $10 \mathrm{~dB}$ higher than our equipment limit. cVEMP thresholds at 500, 750 and $1000 \mathrm{~Hz}$ were collected and analyzed.

\section{Data analysis}

An independent $t$ test was performed to compare age of the patient and healthy control groups. Full factorial analysis of variance (ANOVA) was performed to examine differences in CVEMP threshold and low frequency ABG among our four different groups (dehiscent versus thin versus unaffected versus healthy control) and to evaluate the influence of stimulus frequency on cVEMP threshold and ABG. Group and frequency were considered fixed factors, while subject was considered a random factor. To compare groups and frequencies, pairwise comparisons were performed using a Bonferroni adjustment for multiple comparisons. To evaluate the ability of cVEMP thresholds to distinguish dehiscent from intact superior semicircular canals, receiver operating characteristic (ROC) curves were generated. To evaluate the value of the ABG and cVEMP together a combined "third window indicator" (TWI) was used. The combined TWI is calculated by subtracting the ABG at $250 \mathrm{~Hz}$ from the CVEMP threshold measured in the same ear (Figure 3.1.3). Statistical analyses were performed using SPSS (version 22.0; Chicago, IL). A $p$ value of $<0.05$ was considered statistically significant. 

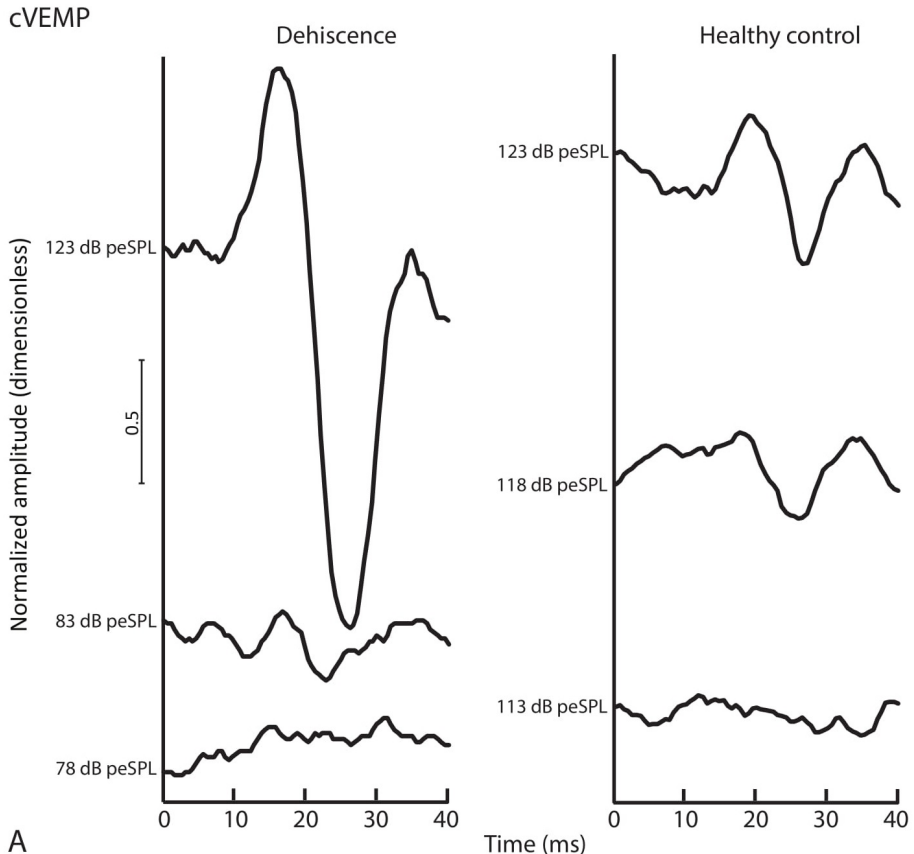

A

$$
\text { Time (ms) }
$$

\section{Audiogram}

Frequency $(\mathrm{Hz})$
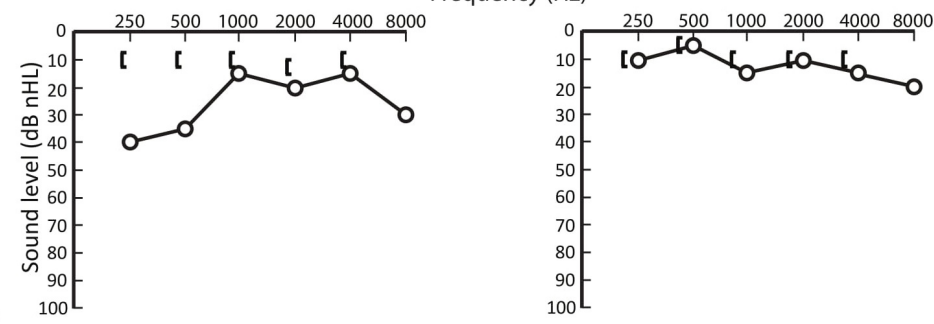

Calculations combined "Third Window Indicator" (TWI)

1) CVEMP threshold $500 \mathrm{~Hz}(\mathrm{~A})=83 \mathrm{~dB}$ peSPL

2) $A B G 250 \mathrm{~Hz}(B)=40-10=30 \mathrm{~dB} \mathrm{nHL}$

1) cVEMP threshold $500 \mathrm{~Hz}(\mathrm{~A})=118 \mathrm{~dB}$ peSPL

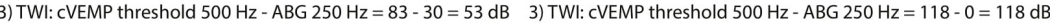

C

Figure 3.1.3. Determination of the combined "third window indicator" (TWI). The TWI was calculated using two examples: a dehiscent (left part figure) and healthy control (right part figure) ear. The cVEMP threshold (A) is subtracted by the ABG at $250 \mathrm{~Hz}(B)$ to calculate the TWI (C). Panel C shows how calculations were performed. cVEMP indicates cervical vestibular evoked myogenic potential; $A B G$, air-bone gap. 


\section{Results}

\section{Patient characteristics}

A total of 140 patients (239 ears) with an average age of 48.6 (range: 15-77) years were included ( 80 women, 60 men). CT imaging demonstrated that 142 ears were dehiscent, 56 were thin and 41 were unaffected. Fifty-one patients had unilateral SCD and 89 patients had bilateral SCD (patients with one dehiscent and one thin side were considered to have bilateral disease). The age-matched control group consisted of 21 healthy subjects (42 ears) with a mean age of 48.9 (range: $32-68$ ) years (12 women, 9 men). No significant difference in age between the patient and control group was found $(p=0.917)$.

\section{Audiometric data}

Air-bone gaps were calculated at 250 and $500 \mathrm{~Hz}$ for all four groups. Averages and variances are displayed in Table 3.1.1 and Figure 3.1.4A. Average ABGs at $250 \mathrm{~Hz}$ were larger than at $500 \mathrm{~Hz}$ for all groups (Figure 3.1.4A). There was a significant effect of group $(F=88.440, p<0.001)$ and frequency $(F=33.753, p<0.001)$. No significant interaction between group and frequency was found $(F=1.791, p=0.148)$. On average, ABGs of dehiscent ears were larger than all three other groups. Pairwise comparisons revealed a significant difference between dehiscent ears and all three other groups (thin $p<0.001$; unaffected $p<0.001$; healthy control $p<0.001$ ). No significant differences were found for the other combinations (thin vs. unaffected $p=1.00$; thin vs. healthy control $p=1.00$; unaffected vs. healthy control $p=1.00$ ).

Table 3.1.1 Mean and 95\% confidence intervals for air-bone gap at 250 and $500 \mathrm{~Hz}$, and cVEMP thresholds at 500,750 and $1000 \mathrm{~Hz}$.

\begin{tabular}{|c|c|c|c|c|c|}
\hline & Frequency $(\mathrm{Hz})$ & $\begin{array}{l}\text { Healthy control } \\
n=42\end{array}$ & $\begin{array}{l}\text { Unaffected } \\
n=41\end{array}$ & $\begin{array}{l}\text { Thin } \\
\mathrm{n}=56\end{array}$ & $\begin{array}{c}\text { Dehiscent } * \\
n=142\end{array}$ \\
\hline \multirow[t]{2}{*}{$\begin{array}{l}\text { Air-bone gap } \\
(\mathrm{dB} n \mathrm{HL})\end{array}$} & 250 & $\begin{array}{c}3.9 \\
(2.3-5.6)\end{array}$ & $\begin{array}{c}2.5 \\
(0.3-4.7)\end{array}$ & $\begin{array}{c}4.4 \\
(2.5-6.3)\end{array}$ & $\begin{array}{c}20.5 \\
(18.1-22.9)\end{array}$ \\
\hline & 500 & $\begin{array}{c}-0.3 \\
(-2.3-1.7)\end{array}$ & $\begin{array}{c}-0.6 \\
(-2.5-1.2)\end{array}$ & $\begin{array}{c}1.8 \\
(0-3.6)\end{array}$ & $\begin{array}{c}13.2 \\
(11.2-15.2)\end{array}$ \\
\hline \multirow[t]{3}{*}{$\begin{array}{l}\text { cVEMP threshold } \\
\text { (dB peSPL) }\end{array}$} & 500 & $\begin{array}{c}114.7 \\
(112.5-116.8)\end{array}$ & $\begin{array}{c}117.0 \\
(114.1-119.9)\end{array}$ & $\begin{array}{c}114.3 \\
(111.7-116.8)\end{array}$ & $\begin{array}{c}98.3 \\
(96.3-100.3)\end{array}$ \\
\hline & 750 & $\begin{array}{c}113.7 \\
(111.8-115.6)\end{array}$ & $\begin{array}{c}118.0 \\
(115.3-120.7)\end{array}$ & $\begin{array}{c}115.6 \\
(113.1-118.1)\end{array}$ & $\begin{array}{c}98.7 \\
(96.8-100.5)\end{array}$ \\
\hline & 1000 & $\begin{array}{c}116.9 \\
(115.1-118.7)\end{array}$ & $\begin{array}{c}120.6 \\
(117.7-123.4)\end{array}$ & $\begin{array}{c}118.2 \\
(116.0-120.4)\end{array}$ & $\begin{array}{c}101.0 \\
(99.1-102.8)\end{array}$ \\
\hline
\end{tabular}

* Note that all values for air-bone gap and cVEMP threshold in the dehiscent group are significantly different from the other three cohorts. cVEMP indicates cervical vestibular evoked myogenic potential. 

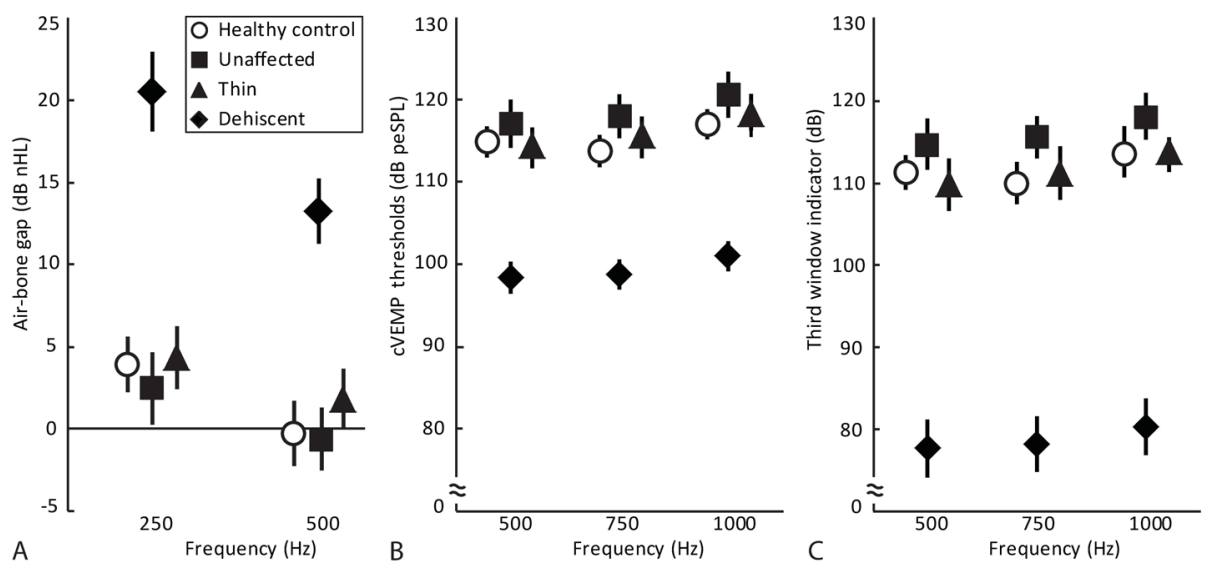

Figure 3.1.4 Average air-bone gap (ABG) at $250 \mathrm{~Hz}$ and $500 \mathrm{~Hz}$ (A), cVEMP thresholds for 500, 750 and $1000 \mathrm{~Hz}$ (B) and Third Window Indicator (TWI) (C). Error bars represent the $95 \%$ confidence intervals. ABG was significantly higher for the SCD group compared with unaffected, thin, and healthy controls. There was no significant difference among unaffected, thin and healthy control ears (A). cVEMP thresholds are statistically significantly different for all pairwise comparisons except for thin vs. healthy control and thin vs. unaffected (B).

\section{cVEMP thresholds}

The mean and variance of the CVEMP thresholds at 500,750 and $1000 \mathrm{~Hz}$ for all four groups are displayed in Table 3.1.1 and Figure 3.1.4B. A significant difference in CVEMP thresholds was found for group $(F=206.647, p<0.001)$ and frequency $(F=7.171$, $p=0.001)$. There was no significant interaction between these two factors $(F=0.145$, $p=0.990)$. Pairwise comparisons revealed significant differences between the following groups: dehiscent vs. thin $(p<0.001)$, dehiscent vs. unaffected $(p<0.001)$, dehiscent vs. healthy control $(p<0.001)$ and unaffected vs. healthy control $(p=0.044)$. We did not observe significant differences in CVEMP threshold for the following comparisons: thin vs. unaffected $(p=0.210)$ and thin vs. healthy control $(p=1.00)$. Frequency pairwise comparisons revealed significant differences between CVEMP thresholds at 1000 and $500 \mathrm{~Hz}(p=0.001)$, and 1000 and $750 \mathrm{~Hz}(p=0.009)$. No significant differences were found between 500 and $750 \mathrm{~Hz}(p=1.00)$.

\section{Diagnostic ability}

Both the $A B G$ at $250 \mathrm{~Hz}$ and cVEMP thresholds were significantly different in dehiscent SSCs compared to all other groups (Figure 3.1.4A and 3.1.4B). We hypothesized that combining these two metrics would improve our ability to differentiate dehiscent from healthy or intact SSCS. Therefore, we created a combined "third window indicator" 
(TWI), which is calculated by subtracting the ABG at $250 \mathrm{~Hz}$ from the cVEMP threshold at 500,750 or $1000 \mathrm{~Hz}$ (Figure 3.1.3 and 3.1.4C).

\section{ROC curves}

Receiver operating characteristic (ROC) curves reveal that the ABG at $250 \mathrm{~Hz}$ and cVEMP thresholds can be used to distinguish dehiscent SCCs from healthy control SSCS with areas under the curves (AUC) of $0.84,0.87,0.86$ and 0.88 for $A B G$ at $250 \mathrm{~Hz}$ and cVEMP threshold at 500, 750 and $1000 \mathrm{~Hz}$, respectively (Figure 3.1.5). Using the TWI improved the ability to distinguish SCD from controls for all frequencies with AUCS increasing to $0.93,0.90$ and 0.92 for 500,750 and $1000 \mathrm{~Hz}$, respectively (Figure 3.1.5). Based on the ROC curves, the best cutoff value (TWI using $500 \mathrm{~Hz}$ cVEMP threshold) to determine whether a SSC is dehiscent or not is $103 \mathrm{~dB}$. This cutoff corresponds to the point in the ROC curve where sensitivity is 0.82 and false positive rate is 0 (Figure 3.1.5). The corresponding positive predictive value for this cutoff value is 1 , meaning that all subjects with a positive test (TWI at $500 \mathrm{~Hz}<103 \mathrm{~dB}$ ) have a dehiscent SCC on CT. The corresponding negative predictive value is 0.59 , indicating that $59 \%$ of the patients with a negative test did not have a dehiscence.

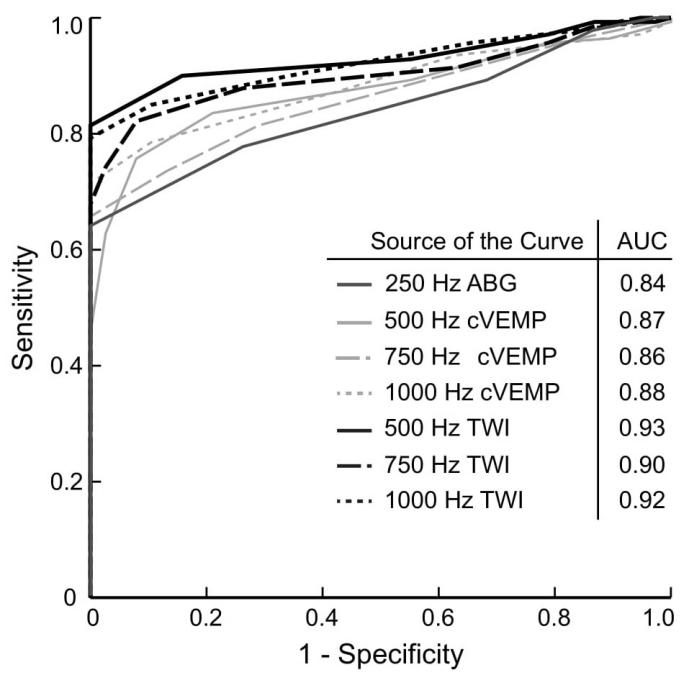

Figure 3.1.5 Receiver operating characteristic (ROC) curves displaying false positive rate (1 - specificity) against sensitivity of detecting a dehiscence for the air-bone gap (ABG) at $250 \mathrm{~Hz}$ only (dark grey line), the cVEMP threshold at 500, 750 and $1000 \mathrm{~Hz}$ only (light gray lines) and for the combined third window indicator (TWI), which is the CVEMP threshold subtracted by the ABG at $250 \mathrm{~Hz}$ (black lines; see Figure 3.1.3 for an example of how the TWI was calculated). The area under the curve (AUC) of every line is displayed. Combining the cVEMP threshold and $250 \mathrm{~Hz}$ $A B G$ to calculate and use the TWI results in higher AUCs for all frequencies. 


\section{Discussion}

To our knowledge, this is the largest study to date that analyzes CVEMP data in SCD patients. We found that the combination of cVEMP thresholds with low frequency ABG can successfully predict the presence of a dehiscent superior semicircular canal. Specifically, these two tests can distinguish between complete dehiscence and normal bone or near dehiscence (thin bone) as defined by dedicated CT imaging. To this end, our third window indicator or TWI (combining data from CVEMP threshold testing and the magnitude of the $A B G$ ) can serve as an effective screening tool for SCD.

Previous studies have investigated the sensitivity and specificity of CVEMP outcomes to predict SCD. In a study of 29 surgically confirmed SCD patients, a cVEMP threshold of 75 $\mathrm{dB} \mathrm{nHL}$ yielded a sensitivity of $57 \%$ and specificity $100 \%$. Ultimately, a higher cutoff ( 85 $\mathrm{dB} \mathrm{nHL}$ ) was established, resulting in a final sensitivity of $86 \%$ and specificity of $90 \%$ (11). A limitation of that study was that it did not combine cVEMP thresholds with ABGs, which may improve the ability to distinguish dehiscent from unaffected ears (10). For example, in 2013, Milojcic et al. found that combining cVEMP thresholds and ABG from the same frequency increased positive predictive values to $68 \%$ at $250 \mathrm{~Hz}, 71 \%$ at $500 \mathrm{~Hz}$ and $66 \%$ at $1000 \mathrm{~Hz}$. These findings corroborate our results, which showed that combining CVEMP thresholds with ABG provides a more accurate method for differentiating dehiscent from healthy SSC. In the present study we achieved a positive predictive value of $100 \%$ by using our combined "TWI" (cVEMP threshold at $500 \mathrm{~Hz}$ $A B G$ at $250 \mathrm{~Hz})$.

There are multiple explanations for this improvement. First, Milojcic et al. corrected the cVEMP threshold for ABG at the same corresponding frequency (e.g. cVEMP thresholds at $500 \mathrm{~Hz}$ were subtracted by $A B G s$ at $500 \mathrm{~Hz}$ ) (10). We have shown that the difference in $A B G$ between dehiscent and healthy control ears is largest at $250 \mathrm{~Hz}$ (Figure 3.1.4A), therefore we chose to combine cVEMP thresholds at all frequencies with $A B G$ of the same ear at $250 \mathrm{~Hz}$ (dubbed the combined "third window indictor", or TWI). This considerably improved our ability to separate dehiscent from healthy ears. Second, Milojcic's study did not specify whether the dehiscent group included patients with a true dehiscence only or also patients with thin bone overlying the SSC. This may represent a crucial confounder as we have shown that both cVEMP thresholds and ABG are significantly different between these two groups. In fact, SSC with thin overlying bone behave more like healthy ears on testing. This is consistent with previous studies, which have also suggested that CVEMP thresholds are significantly different between dehiscent (47 ears) and thin bone (17 ears) (7).

Overall, our ROC curves showed that the $500 \mathrm{~Hz}$ TWI (cVEMP threshold at $500 \mathrm{~Hz}$ subtracted by $A B G$ at $250 \mathrm{~Hz}$ ) gave the best combination of sensitivity and false positive rate, and the optimal cutoff value was $103 \mathrm{~dB}$. At this value, the maximum possible positive predictive value improved from $71 \%$ (found by Milojcic et al. 2013) (10) to $100 \%$ in our study. 
Interestingly, when comparing CVEMP thresholds of our four cohorts, CVEMP thresholds of the unaffected ears in unilateral SCD patients had significantly higher thresholds than our healthy control group, whereas cVEMP thresholds of the thin group were comparable to the healthy control group (Figure 3.1.4B). It is unclear why this difference exists, and to our knowledge this is the first study that demonstrates this phenomenon. We theorize that in patients with unilateral SCD, there may be central suppression of the saccular response to sound on both sides as a form of protection and compensation for the hyperactivation of the saccule in the affected ear; this would then lead to the observed increase in CVEMP threshold in the contralateral ear. These are only speculations, and future studies are warranted to further investigate this relationship.

When comparing our thin group and healthy control group, we did not find significant differences in either the ABG at low frequencies or cVEMP thresholds. Therefore, CT imaging would be an important tool for the differentiation of these two groups. However, when distinguishing thin from dehiscent bone, cVEMP could be an important addition, especially given that CT imaging is not $100 \%$ accurate in differentiating these two groups. In fact, previous cadaveric and clinical studies have shown that CT imaging overestimates the presence of SCD and may misdiagnose a dehiscence in about one third of cases when there is actually thin bone overlying the SSC $(12,22-24)$. The use of higher resolution (e.g. cone beam) CT scans has been suggested to more accurately detect the presence of a dehiscence (25-27). The use of ABG and CVEMP would be useful in this situation because they reflect actual physiological measurements.

We recommend that the TWI that combines cVEMP threshold at $500 \mathrm{~Hz}$ with the ABG at $250 \mathrm{~Hz}$ be used as a screening tool for patients with SCD symptoms (Figure 3.1.6). If the test is positive and the patient does not have severity of symptoms that warrant surgery, a CT scan could be deferred for a later date if the clinical course worsens. However, a CT scan is certainly mandatory as part of pre-operative assessment in patients undergoing surgical treatment of their dehiscence. In case of a negative test, CT imaging could be considered, given our sensitivity of $82 \%$ and a negative predictive value of $59 \%$. Particularly, one must be cautious when using CT imaging to differentiate dehiscent from thin for reasons previously mentioned.

Furthermore, it must be emphasized that patients with an $A B G>10 \mathrm{~dB}$ at any of the tested frequencies plus abnormal tympanograms and/or acoustic reflexes may also have middle ear pathology. In such cases the cVEMP cannot be reliably interpreted. Therefore, temporal bone CT imaging is indicated to evaluate the SSC (Figure 3.1.6). Because the CVEMP is not a standardized test and methods can vary by institution, every institution should validate their own optimal cutoff value (demonstrated to be $103 \mathrm{~dB}$ at our site). 


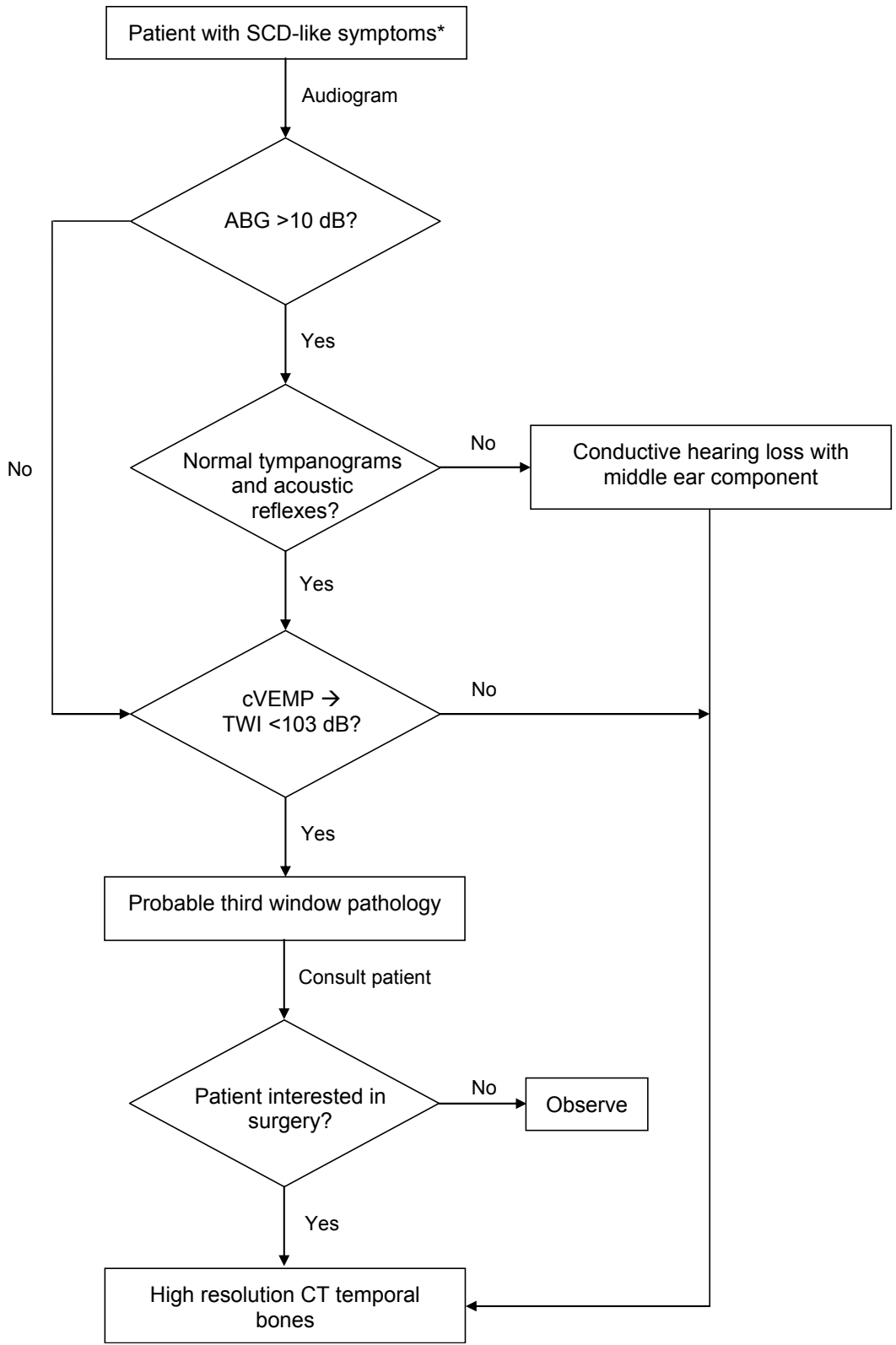

Figure 3.1.6 Flowchart displaying our proposed clinical algorithm in the evaluation of patients with SCD-like symptoms. *SCD-like symptoms may include: autophony, hyperacusis, aural fullness, tinnitus, hearing loss, sound or pressure induced dizziness. ABG indicates air-bone gap; TWI, combined third window indicator. 


\section{Limitations}

A limitation of this study is that assignment of our patient groups was based solely on the subjective evaluation of their CT scans by experienced neuroradiologists and these images varied in quality. Since most of these subjects did not undergo exploration of the skull base, the presence of a bony defect (dehiscent or thin) was not surgically confirmed. Therefore, our categorization of dehiscent and thin groups may not always be accurate and it is possible that patients who were categorized as dehiscent actually had thin bone covering the SSC. It has previously been suggested that the CVEMP might be more reliable than $\mathrm{CT}$ imaging in detecting a dehiscence and we could theorize that our sensitivity of $82 \%$, which corresponds to a false negative rate of $18 \%$, is due to a misdiagnosis of dehiscence when patients actually had thin bone covering the SSC (12). Regarding differences in CT technology, we compared CVEMP and ABG outcomes of our study by stratifying the data as a function of location where CT imaging was performed (either in our institution or in an outside institution). We found no significant differences in our results (data not shown). Finally, the use of cVEMP threshold combined with ABG needs to be prospectively validated as a screening tool, which will be the subject of future studies.

\section{Conclusion}

Combining cVEMP threshold at $500 \mathrm{~Hz}$ with the audiometric low frequency ABG at $250 \mathrm{~Hz}$ improves the ability to detect SCD. Our "third window indicator" or TWI enhances the clinical applicability of CVEMP and is a robust screening tool for diagnosing SCD without the need for imaging, especially in patients who have mild or moderate symptoms and are not surgical candidates. 


\section{References}

1. Minor LB, Solomon D, Zinreich JS, et al. Sound- and/or pressure-induced vertigo due to bone dehiscence of the superior semicircular canal. Arch Otolaryngol Head Neck Surg 1998;124:249-58.

2. Rosowski JJ, Songer JE, Nakajima HH, et al. Clinical, experimental, and theoretical investigations of the effect of superior semicircular canal dehiscence on hearing mechanisms. Otol Neurotol 2004;25:323-32.

3. Ho ML, Moonis G, Halpin CF, et al. Spectrum of Third Window Abnormalities: Semicircular Canal Dehiscence and Beyond. AJNR Am J Neuroradiol 2017;38:2-9.

4. Crane BT, Carey JP, McMenomey S, et al. Meningioma causing superior canal dehiscence syndrome. Otol Neurotol 2010:31:1009-10.

5. Rauch SD, Zhou G, Kujawa SG, et al. Vestibular evoked myogenic potentials show altered tuning in patients with Meniere's disease. Otol Neurotol 2004; 25:333-8.

6. Rauch SD, Silveira MB, Zhou G, et al. Vestibular evoked myogenic potentials versus vestibular test battery in patients with Meniere's disease. Otol Neurotol 2004;25:981-6.

7. Mehta R, Klumpp ML, Spear SA, et al. Subjective and objective findings in patients with true dehiscence versus thin bone over the superior semicircular canal. Otol Neurotol 2015;36:289-94.

8. Hunter JB, Patel NS, O'Connell BP, et al. Cervical and Ocular VEMP Testing in Diagnosing Superior Semicircular Canal Dehiscence. Otolaryngol Head Neck Surg 2017;156:917-23.

9. Benamira LZ, Alzahrani M, Saliba I. Superior canal dehiscence: can we predict the diagnosis? Otol Neurotol 2014;35:338-43.

10. Milojcic R, Guinan JJ Jr, Rauch SD, et al. Vestibular evoked myogenic potentials in patients with superior semicircular canal dehiscence. Otol Neurotol 2013; 34:360-7.

11. Zuniga MG, Janky KL, Nguyen KD, et al. Ocular versus cervical VEMPs in the diagnosis of superior semicircular canal dehiscence syndrome. Otol Neurotol 2013;34:121-6.

12. Ward BK, Wenzel A, Ritzl EK, et al. Near-dehiscence: clinical findings in patients with thin bone over the superior semicircular canal. Otol Neurotol 2013;34:1421-8.

13. Colebatch JG, Halmagyi GM, Skuse NF. Myogenic potentials generated by a click-evoked vestibulocollic reflex. J Neurol Neurosurg Psychiatry 1994;57:190-7.

14. Govender S, Fernando T, Dennis DL, et al. Properties of $500 \mathrm{~Hz}$ air- and bone-conducted vestibular evoked myogenic potentials (VEMPs) in superior canal dehiscence. Clin Neurophysiol 2016;127:2522-31.

15. Roditi RE, Eppsteiner RW, Sauter TB, et al. Cervical vestibular evoked myogenic potentials (cVEMPs) in patients with superior canal dehiscence syndrome (SCDS). Otolaryngol Head Neck Surg 2009;141:24-8.

16. Brantberg K, Verrecchia L. Testing vestibular-evoked myogenic potentials with $90-\mathrm{dB}$ clicks is effective in the diagnosis of superior canal dehiscence syndrome. Audiol Neurootol 2009;14:54-8.

17. Welgampola MS, Myrie OA, Minor LB, et al. Vestibular-evoked myogenic potential thresholds normalize on plugging superior canal dehiscence. Neurology 2008;70:464-72.

18. Streubel SO, Cremer PD, Carey JP, et al. Vestibular-evoked myogenic potentials in the diagnosis of superior canal dehiscence syndrome. Acta Otolaryngol Suppl 2001;545:41-9.

19. Brantberg K, Bergenius J, Tribukait A. Vestibular-evoked myogenic potentials in patients with dehiscence of the superior semicircular canal. Acta Otolaryngol 1999;119:633-40.

20. Standards AN. ANSI S3.21-2004 Methods for Manual Pure-Tone Threshold Audiometry. New York, NY: Acoustical Society of America, 2004b.

21. Standards AN. ANSI S3.6-2004 Specification for Audiometers. New York, NY: Acoustical Society of America, 2004a.

22. Sequeira SM, Whiting BR, Shimony JS, et al. Accuracy of computed tomography detection of superior canal dehiscence. Otol Neurotol 2011;32:1500-5.

23. Tavassolie TS, Penninger RT, Zuñiga MG, et al. Multislice computed tomography in the diagnosis of superior canal dehiscence: how much error, and how to minimize it? Otol Neurotol 2012;33:215-22.

24. Re M, Gioacchini FM, Salvolini $U$, et al. Multislice computed tomography overestimates superior semicircular canal dehiscence syndrome. Ann Otol Rhinol Laryngol 2013;122: 625-31.

25. Sepulveda I, Schmidt T, Platin E. Use of cone beam computed tomography in the diagnosis of superior semicircular canal dehiscence. J Clin Imaging Sci 2014;4:49. 
26. Eibenberger K, Carey J, Ehtiati T, et al. A novel method of 3D image analysis of high-resolution cone beam CT and multi slice CT for the detection of semicircular canal dehiscence. Otol Neurotol 2014;35:329-37.

27. Penninger RT, Tavassolie TS, Carey JP. Cone-beam volumetric tomography for applications in the temporal bone. Otol Neurotol 2011; 32:453-60. 



\title{
Chapter
}

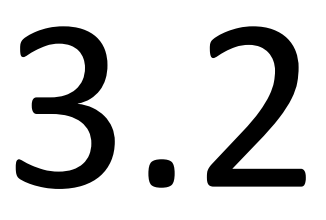

\section{Audiometric and cVEMP thresholds show little correlation with symptoms in superior semicircular canal dehiscence syndrome}

\author{
KS Noij \\ K Wong \\ MJ Duarte \\ S Masud \\ NA Dewyer \\ BS Herrmann \\ JJ Guinan Jr. \\ ED Kozin \\ DH Jung \\ SD Rauch \\ DJ Lee
}




\section{Abstract}

\section{Objective}

Evaluate the relationship between objective audiometric and vestibular tests and patient symptoms in superior canal dehiscence syndrome (SCD).

\section{Study design}

Retrospective chart review

\section{Setting}

Tertiary care center

\section{Patients}

Ninety-eight patients with SCD and available preoperative threshold audiograms, cervical vestibular evoked myogenic potential (cVEMP) thresholds, and computed tomography (CT) imaging were included. Clinical reports were reviewed for selfreported SCD symptoms. Twenty-five patients completed the Hearing Handicap Inventory (HHI), Dizziness Handicap Inventory (DHI), Autophony Index (AI) and the 36item Short Form Survey (SF-36).

\section{Main outcome measures}

Correlations between preoperative low-frequency air-bone gap (ABG), CVEMP thresholds and symptoms (including $\mathrm{HHI}, \mathrm{DHI}, \mathrm{Al}$ and SF-36). Symptoms included: hearing loss, aural fullness, autophony, hyperacusis, tinnitus, vertigo, imbalance and sound-, pressure and exercise provoked dizziness. Secondary outcome measure: Correlations between changes of objective and subjective measures before and after surgery.

\section{Results}

Patients who reported hearing loss had larger ABGs at $250 \mathrm{~Hz}$ than patients without subjective hearing loss $(p=0.001)$. ABG and cVEMP threshold did not correlate with any other symptom. No significant correlation was found between ABG or CVEMP threshold and the $\mathrm{HHI}, \mathrm{DHI}, \mathrm{Al}$ or Health Utility Value (derived from the SF-36 quality of life score). Following SCD surgery, ABG decreased $(p<0.001)$, cVEMP thresholds increased $(p<0.001)$ and overall symptoms, handicap scores and quality-of-life improved; however, there was no significant relationship between these measures.

\section{Conclusion}

While threshold audiometry and CVEMP are important tools to diagnose SCD and monitor surgical outcomes, these measures showed no significant correlation with vestibular and most auditory symptoms or their severity. 


\section{Introduction}

Semicircular canal dehiscence syndrome (SCD) is a condition characterized by a bony defect in the superior semicircular canal (SSC) that can lead to a host of auditory and vestibular symptoms, including hearing loss, aural fullness, autophony, hyperacusis, tinnitus, imbalance and sound-, pressure- or exercise-induced dizziness (1-3). In SCD, a dehiscence produces a "third window" that leads to shunting of acoustic energy away from the cochlear partition toward the bony defect of the superior canal. Thus, for any given amount of air-conducted sound energy delivered to an SCD ear, the cochlea receives less sound energy and the vestibular sense organs receive comparatively more energy. The third window also influences bone-conducted hearing thresholds, although this mechanism is less clear $(4,5)$. Objective tests to assess the auditory and vestibular systems include threshold audiometry and cervical vestibular evoked myogenic potentials (cVEMPs).

cVEMPs are commonly used to assess patients with SCD and Menière's disease (6-10). The CVEMP is an indirect measure of saccular and inferior vestibular nerve function. During a cVEMP measurement, the saccule is acoustically or mechanically stimulated and produces inhibition of the ipsilateral sternocleidomastoid muscle as measured by electromyography (EMG) (11). Compared to healthy subjects, SCD patients typically show lower cVEMP thresholds, higher amplitudes and low frequency air-bone gaps (ABG) $(9,10,12-16)$.

Although cVEMP thresholds and $A B G s$ provide important data regarding the physiologic changes of the inner ear with SCD, few studies have investigated the relationship of these objective metrics with symptoms reported by patients with SCD $(8,14,16-18)$. Two small series ( $n=3$ and $n=7$ ) found that in patients with bilateral $S C D$, the more symptomatic ear had lower cVEMP thresholds $(17,18)$. Another study found that asymptomatic ears with a dehiscence $(n=5)$ had cVEMP amplitudes and thresholds similar to non-dehiscent ears (14). Together, these early findings suggest a possible relationship between CVEMP thresholds and symptomatology and underscore the need for further investigation.

Following SCD surgery, preoperative diagnostic indicators tend to normalize (cVEMP thresholds increase, ABGs decrease, and auditory and vestibular symptoms as well as quality of life improve) $(17,19-21)$. While these changes have been studied individually, little data exists regarding the correlation between cVEMP thresholds and ABGs with symptoms and quality of life measures. In the present study, we test the hypothesis that auditory symptoms are associated with larger ABGs and vestibular symptoms are associated with lower cVEMP thresholds in patients with SCD. Our second objective was to investigate whether the normalization of ABG and CVEMP thresholds after surgery coincide with improvements in symptoms. 


\section{Methods}

\section{Subjects}

We performed a retrospective chart review of data collected in our institutions' SCD database (2000-2016). Patients with available audiometric, cVEMP and computed tomography (CT) imaging data as well as clinical data on symptomatology, including validated questionnaires, were included for analysis (22-25). Patients with existing middle ear pathology were excluded.

\section{Radiology}

Patients underwent CT imaging to determine the presence and location of the bony superior canal defect. Imaging data included multiplanar reconstruction in the plane of Stenvers (perpendicular to the plane of the SSC) and Pöschl (parallel to SSC). All scans were reviewed by a neuroradiologist at our institution. Only patients with a "true dehiscence" (a visible aperture in the bone, as opposed to "thin" or "near" dehiscence) on CT imaging and the presence of symptoms were included (16). The majority of patients underwent imaging at our institution. CT scans were obtained using either a multidetector row CT scanner (Somatom Sensation 40; Siemens Healthcare, Erlangen, Germany or, after 2014 Discovery CT750 HD; GE Healthcare, Milwaukee, WI) or a cone beam CT scanner (3D Accuitomo 170; J. Morita, Irvine, CA) that generated images with a slice thickness of 0.63 and $0.5 \mathrm{~mm}$, respectively. The axial pixel dimension of the cone beam scans was $0.125 \mathrm{~mm}$. The slice thickness of outside hospital scans in the axial view ranged from 0.5 to $1.25 \mathrm{~mm}$ (mean: $0.68 \mathrm{~mm}$ ).

\section{Audiometric data}

All subjects underwent a comprehensive audiogram, including air- and boneconduction thresholds down to $-10 \mathrm{~dB} \mathrm{HL}$ (hearing level) as well as impedance audiometry $(26,27)$. Bone-conduction thresholds were masked in case of a difference between air- and unmasked bone-conduction thresholds of more than $10 \mathrm{~dB} \mathrm{HL}$. The presence of an $A B G$ at $250 \mathrm{~Hz}$ without signs of middle ear pathology is common in patients with SCD and is helpful in differentiating patients with and without SCD (16). Therefore, ABGs were calculated at $250 \mathrm{~Hz}$ by subtracting the bone-conduction threshold from the air-conduction threshold. If there was an $A B G>10 \mathrm{~dB} H L$ for any of the tested frequencies, patients were evaluated with immittance audiometry to evaluate middle ear function. Ears with $A B G>10 \mathrm{~dB} H \mathrm{HL}$ were only included if tympanograms were normal and acoustic reflexes were present to exclude middle ear pathology. 


\section{CVEMP}

During CVEMP testing, patients sat upright with their head turned away from the stimulated ear to contract the ipsilateral sternocleidomastoid muscle (SCM). Four surface electrodes were placed to record electromyogram (EMG) activity: a positive electrode on the middle belly of each SCM, a reference electrode in the midline of the superior part of the sternum (manubrium), and a ground electrode on the midline forehead. During testing the ipsilateral SCM EMG amplitude was monitored and patients were verbally encouraged to contract their SCM to produce $>65 \mu \mathrm{V}$ root mean square (rms) EMG. EMG activity was amplified, bandpass filtered and sampled at $50 \mathrm{kHz}$ with a 16-bit analog-to-digital converter (National Instruments, Austin, TX). Patients were acoustically stimulated with $500 \mathrm{~Hz}$ tone bursts presented monaurally with circumaural headphones (Telephonics TDH-49) at a repetition rate of 13 bursts/s. Tone bursts were generated by custom-programmed evoked potential software (National Instruments 16-bit digital I/O board) using a Blackman gating function with two cycle $(4.0 \mathrm{~ms})$ rise and fall times and no plateau. cVEMP thresholds were collected and analyzed at $500 \mathrm{~Hz}$. There are small differences in the ability of cVEMP thresholds to distinguish between healthy and dehiscent ears using 500, 750 and $1000 \mathrm{~Hz}$ tone bursts (16). Since $500 \mathrm{~Hz}$ is the most commonly used frequency in cVEMP testing, this frequency was chosen for analysis (28). To obtain the cVEMP threshold, responses were first obtained at $123 \mathrm{~dB}$ peSPL (peak sound pressure level; $123 \mathrm{~dB}$ peSPL is equivalent to $90 \mathrm{~dB} \mathrm{nHL}$ ). Next, the sound level was decreased in steps of $10 \mathrm{~dB}$ until no response was observed. Sound levels were then raised in $5 \mathrm{~dB}$ increments and the lowest sound level at which a cVEMP was present was defined as the threshold. If the highest possible stimulus intensity (133 dB peSPL) did not elicit a response, the CVEMP threshold was considered to be $10 \mathrm{~dB}$ above the $133 \mathrm{~dB}$ peSPL limit. Previously obtained CVEMP data from healthy controls in a similar age range as our patient population revealed a median $500 \mathrm{~Hz}$ cVEMP threshold of $113.0 \mathrm{~dB}$ peSPL (IQR: 111.8-118.0) (16). Although the current study does not focus on differences in CVEMP thresholds between patients and healthy controls, these normal values provide a reference for the SCD cVEMP data.

\section{Symptomatology and patient-reported outcomes}

Medical records and prospectively administered questionnaires were used to identify auditory symptoms (hearing loss, aural fullness, autophony, hyperacusis, tinnitus) and vestibular symptoms (vertigo, imbalance and sound-, pressure- and exercise-provoked dizziness) specific to the ear(s) with SCD. Questionnaires provided to patients included the Hearing Handicap Inventory (HHI), Dizziness Handicap Inventory (DHI), Autophony Index (Al) and the 36-item Short Form Survey (SF-36) (22-25). The HHI and DHI range from 0 to 100, with higher scores denoting increased handicap (22-23). The Al ranges from 0 to 104, and higher scores correspond to more bothersome autophony 
symptoms (24). The SF-36 is a 36 -item questionnaire concerning health related quality of life (25). To assess quality of life, the Health Utility Value (HUV) was calculated using an algorithm developed by and used with permission from the Department of Health Economics and Decision Science at the University of Sheffield, UK (29). The HUV is a single value derived from the SF-36 that ranges between 0.3 (poor health) and 1.0 (perfect health) and has previously been used to monitor quality of life changes in patients with SCD (20).

\section{Data analysis}

The Shapiro-Wilk test was used to determine if the data was normally distributed in order to select the appropriate statistical tests. The preoperative ABG $(p=0.041)$, cVEMP threshold $(p<0.001)$ and Al $(p=0.004)$ data were not normally distributed, while the HHI $(p=0.062)$, DHI $(p=0.242)$ and HUV $(p=0.095)$ scores were normally distributed. As a result, the non-parametric Mann-Whitney $U$ test was used to identify differences in ABG and cVEMP thresholds between patients with and without symptoms. The Mann-Whitney $U$ was also used for surgical subjects to assess differences between groups with and without improvement of symptoms in postoperative $A B G$ and cVEMP threshold. The Wilcoxon signed-rank test was used to compare pre- and postoperative ABG, cVEMP threshold and Al. Paired $t$ tests were used to compare the normally distributed pre- and postoperative $\mathrm{HHI}, \mathrm{DHI}$ and HUV. A Bonferroni correction was used to correct for multiple comparisons. To evaluate whether there was a correlation between DHI, HHI, Al or HUV and ABG or CVEMP threshold, Spearman's rank correlation coefficients were calculated. This method was also used to assess the improvement in $\mathrm{DHI}, \mathrm{HHI}, \mathrm{Al}$ and HUV before and after surgery as they relate to changes in the ABG and CVEMP threshold. Statistical analyses were performed using SPSS (version 22.0; Chicago, IL). A $p$ value of $<0.05$ was considered statistically significant unless a Bonferroni correction was used, in which case the significance criterion was 0.05 divided by the number of comparisons. In case of missing data the patient was excluded from relevant analyses. In Table 3.2.1, $\mathrm{n}$ indicates the number of subjects included for analysis in each group.

\section{Results}

\section{Patient demographics}

A total of 98 patients were included (average age: 49.3 years, range: 16-77 years; 42 male, 56 female). Symptomatology data was available for all patients. Twenty-five patients completed the HHI, DHI, Al and SF-36. 


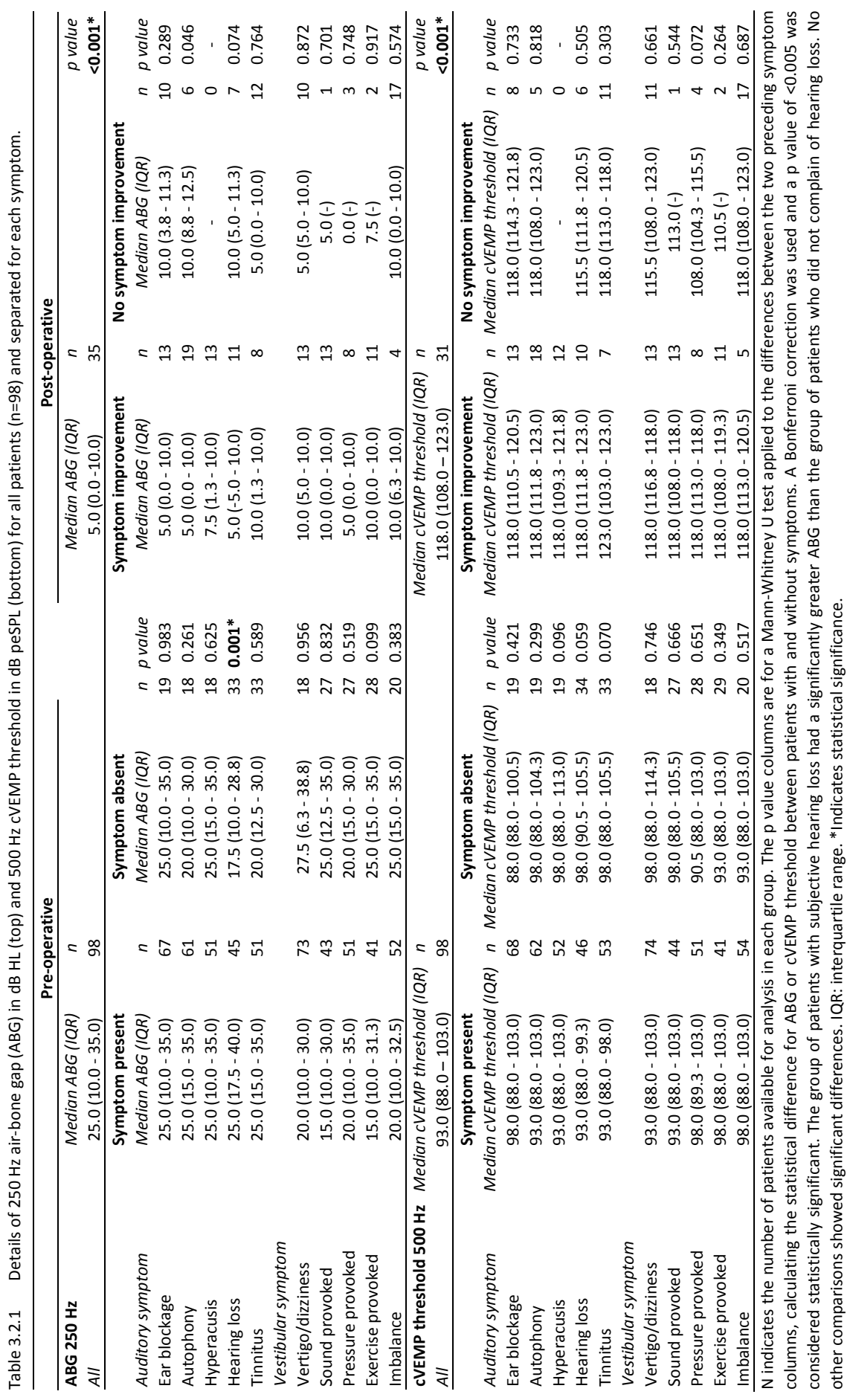


CT imaging demonstrated a unilateral SSC dehiscence in 24 patients, bilateral dehiscence in 37 patients, and one dehiscent ear and one thin/near dehiscent ear in 30 patients. In seven patients the presence or absence of bilateral dehiscence could not be determined because CT imaging was performed on only one side (cone beam CT). Data from the ear with a "true dehiscence" on CT scan were used. In cases of bilateral dehiscence, the more symptomatic side was used for analysis.

In total, 50 patients underwent SCD repair: 47 patients underwent a middle fossa craniotomy (MFC) and three patients underwent a transmastoid approach with fenestration and occlusion of the ascending and descending limbs of the superior canal to isolate the defect. All surgeries involved plugging of the superior semicircular canal with bone wax and most also underwent resurfacing of dehiscent tegmen lateral to the dehiscence with a split calvarial bone graft and temporalis fascia. Thirty-five patients had both postoperative audiograms performed $>3$ months after surgery and information about at least one auditory or vestibular symptom. Thirty-one patients underwent postoperative CVEMP testing. Of the 25 patients who filled out questionnaires preoperatively, 15 underwent surgery and 8 filled out postoperative questionnaires.

\section{Audiometric data and symptoms}

The prevalence of auditory symptoms in our cohort is summarized in Figure 3.2.1A. The preoperative median ABG at $250 \mathrm{~Hz}$ of all patients was $25.0 \mathrm{~dB} \mathrm{HL}$ (IQR: 10.0-35.0; Table 3.2.1). Patients who reported subjective hearing loss had significantly greater ABGs at $250 \mathrm{~Hz}$ compared to patients without this symptom ( $p=0.001$; Table 3.2.1). The magnitude of the ABG at $250 \mathrm{~Hz}$ was not associated with any other auditory symptoms (Table 3.2.1). The median air-conduction threshold of patients reporting subjective hearing loss was greater than $17.5 \mathrm{~dB} \mathrm{HL}$ for all frequencies, while the median air-conduction threshold of patients without subjective hearing loss was less than $17.5 \mathrm{~dB} \mathrm{HL}$ (Figures 3.2.2A and 3.2.2C). Although HHI, Al and HUV scores increased with $A B G$ at $250 \mathrm{~Hz}$ (Figures 3.2.3A-C), these trends were not significant $(\rho=0.474$, $p=0.017 ; \rho=0.253, p=0.223 ; \rho=0.131, p=0.533$ respectively; Bonferroni corrected criterion for statistical significance was a $p$ value of $<0.0167$ ).

Although median preoperative bone-conduction thresholds were not supranormal (defined as a threshold below $0 \mathrm{~dB} \mathrm{HL}$ ), 43\% of patients did show a supranormal $250 \mathrm{~Hz}$ bone-conduction threshold on their audiogram (Figure 3.2.2). No significant differences in bone-conduction threshold were found between patients with and without any of the evaluated symptoms or scores (data not shown). 

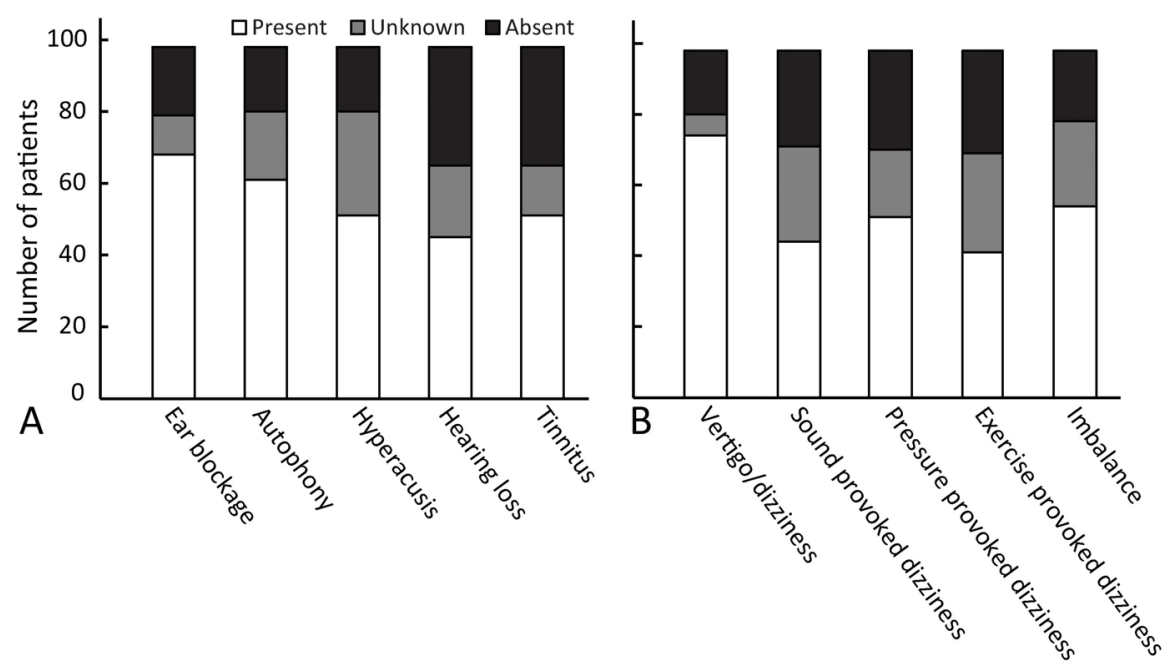

Figure 3.2.1 Histograms summarizing the prevalence of auditory (A) and vestibular (B) symptoms in the 98 included patients. White bars indicate the symptom was present, black bars indicate the symptom was absent and grey bars indicate that it was unknown whether the symptom was present or absent.

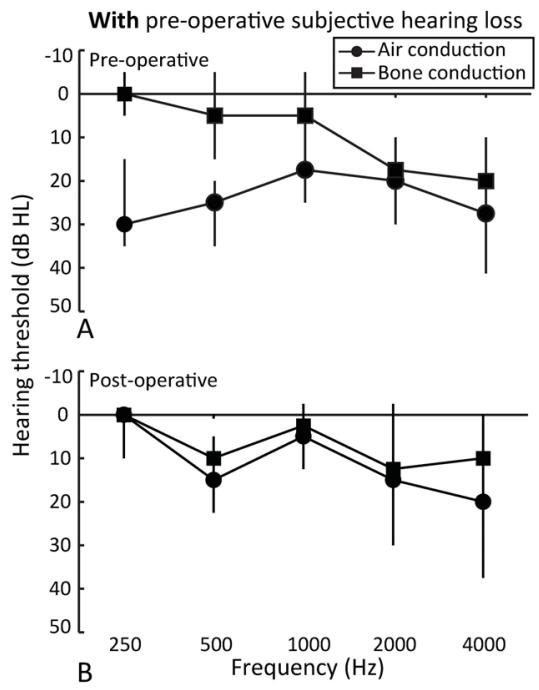

Without pre-operative subjective hearing loss
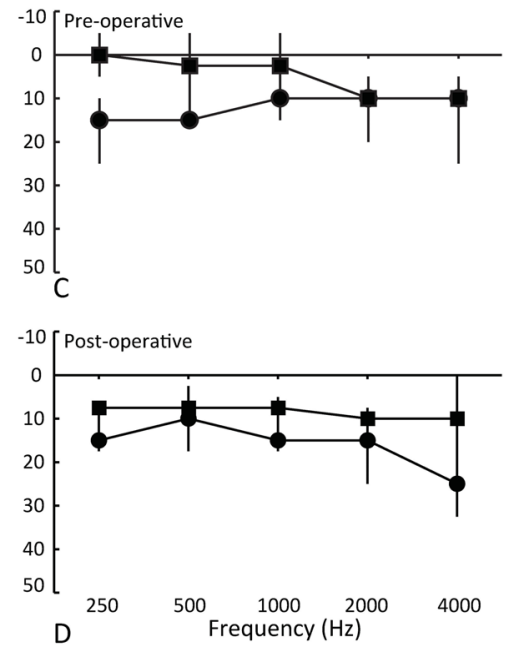

Figure 3.2.2 Median air- (circles) and bone-conduction (squares) hearing thresholds in dB HL. Error bars indicate the interquartile range. Left panels ( $A$ and $B$ ) show data from patients who reported preoperative subjective hearing loss. Their $A B G$ was closed by a decrease in air-conduction threshold. In contrast, the right panels ( $C$ and $D$ ) show results for patients who did not report preoperative subjective hearing loss. Their median preoperative ABG was smaller and it was closed postoperatively mostly by a shift of the bone-conduction threshold. ABG indicates airbone gap. 


\section{cVEMP threshold and symptoms}

The prevalence of vestibular symptoms in our cohort is summarized in Figure 3.2.1B. The median preoperative cVEMP threshold at $500 \mathrm{~Hz}$ was $93.0 \mathrm{~dB}$ peSPL (IQR: 88.0-103.0; Table 3.2.1). There was no significant correlation between CVEMP thresholds and any vestibular symptom (Table 3.2.1). Likewise, neither DHI, Al nor HUV showed a significant correlation with cVEMP thresholds $(\rho=0.036, p=0.864 ; \rho=0.084$, $p=0.689$ and $\rho=-0.046, p=0.827$ respectively; Figure 3.2.3D-F).
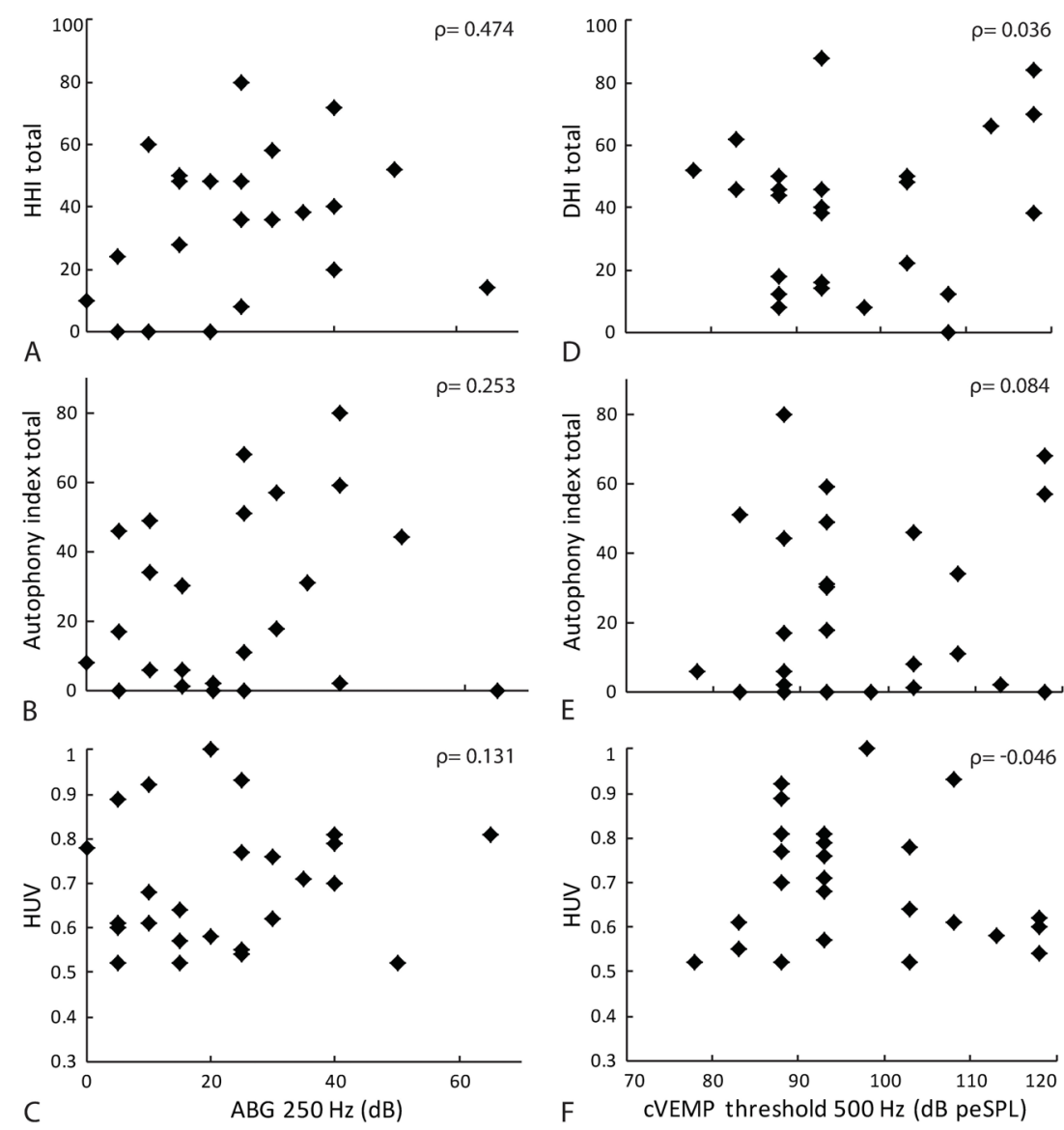

Figure 3.2.3 Scatter plots displaying the correlation between $250 \mathrm{~Hz} A B G$ and Hearing Handicap Inventory (HHI) (A), Autophony Index (AI) (B) and Health Utility Value (HUV) (C) as well as the correlation between $500 \mathrm{~Hz}$ cVEMP and Dizziness Handicap Inventory (DHI) (D), Al (E) and HUV (F). The correlation between the variables are displayed by the $\rho$ value in each panel. Although $\mathrm{HHI}, \mathrm{Al}$ scores and HUV increased with $A B G(A-C)$ these trends were not significant. The correlation between $250 \mathrm{~Hz} A B G$ and $\mathrm{HHI}$ however, was near significant ( $p=0.017$ with a Bonferroni corrected criterion of statistical significance of $p<0.0167$; A). Neither DHI, nor Al or HUV showed a significant correlation with cVEMP threshold (D-F). 


\section{Postoperative data}

The average time between surgery and first postoperative audiogram was 3.9 months (range: 3-8 months). Postoperatively, the $250 \mathrm{~Hz}$ ABG decreased significantly $(p<0.001$; Table 3.2.1) due to both an increase in bone-conduction thresholds and a decrease in air-conduction thresholds (Figure 3.2.2). All but two patients had a decrease in $250 \mathrm{~Hz}$ ABG. It is noteworthy that ABG closure in surgical patients with preoperative subjective hearing loss arose from changes in air-conduction thresholds; i.e. air-conduction threshold decreased. In contrast, in patients with no complaint of preoperative hearing loss, the postoperative ABG was closed mostly by a shift of the bone curve. Overall, patients with improvement of most auditory symptoms had slightly smaller postoperative $250 \mathrm{~Hz}$ ABGs but this difference did not achieve significance, nor were there any significant differences in ABG between patients with or without improvement of vestibular symptoms (Table 3.2.1).

The time between surgery and first postoperative cVEMP ranged from 3-41 months (mean: 5.9 months). The $500 \mathrm{~Hz}$ cVEMP threshold significantly increased after surgery $(p<0.001$; Table 3.2.1). An increase in cVEMP threshold was seen in all but one patient. However, no significant relationship was found between the postoperative CVEMP threshold and improvement of vestibular symptoms (Table 3.2.1).

The HHI, Al and HUV significantly improved after surgery $(p=0.007, p=0.012$ and $p=0.004$ respectively; Figure 3.2.4A, 3.2.4B and 3.2.4D). Although we observed improvement in $\mathrm{DHI}$, this change did not reach significance $(p=0.338$; Bonferroni corrected criterion for statistical significance was a $p$ value of $<0.0125$, Figure 3.2.4C). Overall, $\mathrm{HHI}$ and $\mathrm{Al}$ improved with decrease in $\mathrm{ABG}$ without reaching significance ( $\rho=0.630, p=0.129$ and $\rho=0.698, p=0.123$ respectively). Regarding the correlation with cVEMP thresholds, overall the $\mathrm{DHI}$ and Al decreased with an increase in CVEMP threshold, but no significant correlation was found $(\rho=-0.491, p=0.263$ and $\rho=-0.348$, $p=0.499$ respectively). Furthermore, no significant correlation was found between the change in ABG or cVEMP threshold and improvement in HUV ( $\rho=0.374, p=0.409$ and $\rho=-0.651, p=0.113$ respectively).

\section{Subgroup analysis}

To evaluate whether our inclusion criteria were too broad, a subgroup analysis was performed using stricter criteria than our original inclusion criteria. The subgroup included only patients with a "true" dehiscence by CT scan (see methods) and a $500 \mathrm{~Hz}$ cVEMP threshold < $103 \mathrm{~dB}$ peSPL or a $250 \mathrm{~Hz} A B G>10 \mathrm{~dB}$ (in combination with normal tympanograms and reflexes), and the presence of at least 1 of the following symptoms: hyperacusis, autophony, sound induced vertigo, pressure induced vertigo, and/or pulsatile tinnitus $(30,31)$. Using these more stringent inclusion criteria, $72 / 98$ ears were included and all previously performed analyses were repeated. Outcomes were similar to those found using the entire set of 98 ears (data not shown). 

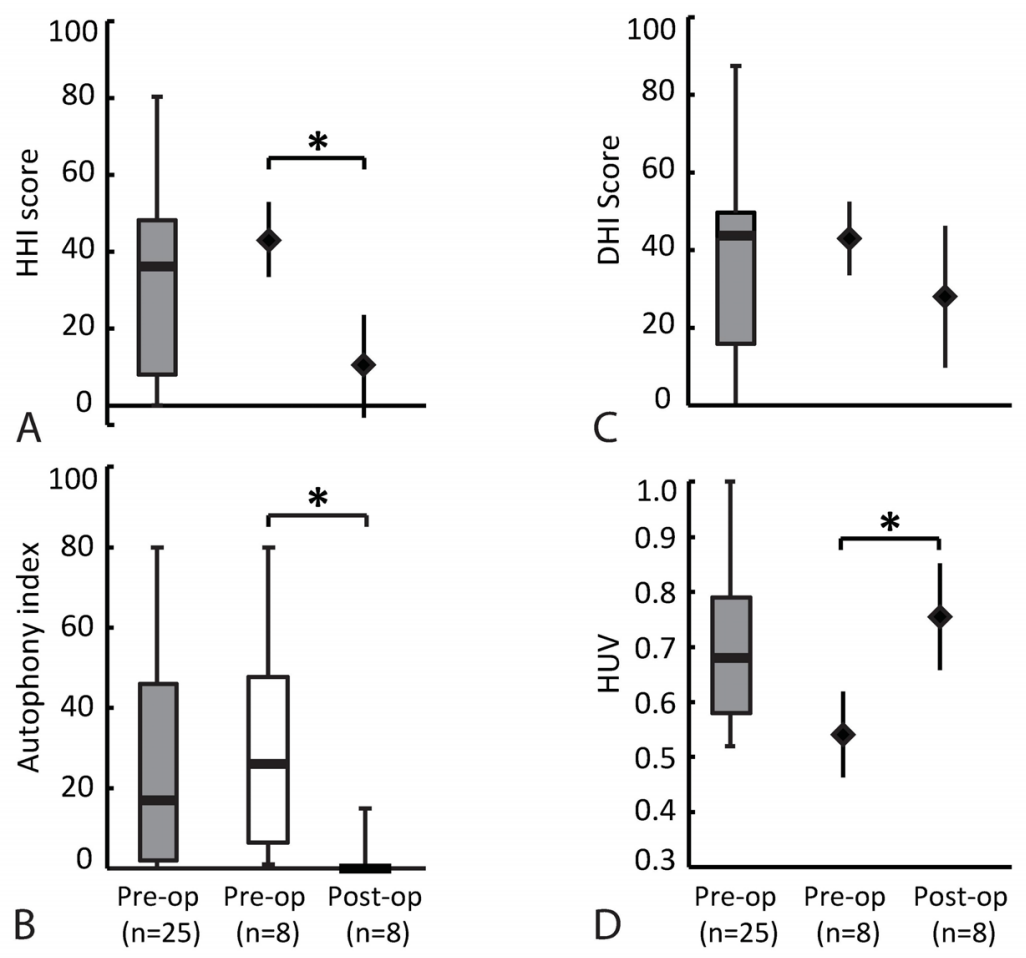

Figure 3.2.4 A comparison of preoperative and postoperative scores. The gray box and whisker plots indicating the minimum, first quartile, median (thick line), third quartile and maximum-score displayed for $\mathrm{HHI}(\mathrm{A}), \mathrm{AI}(\mathrm{B}), \mathrm{DHI}(\mathrm{C})$, and HUV (D) in all patients with preoperative scores available $(n=25)$ to give an indication of the distribution. Diamonds indicate averages of the $\mathrm{HHI}, \mathrm{DHI}$ and HUV scores for patients with both pre- and postoperative data available $(n=8)$. Error bars represent the $95 \%$ confidence intervals. Because our autophony data was not normally distributed white box and whisker plots were used to represent Al scores for patients with both pre- and postoperative data available $(n=8)$. For postoperative $\mathrm{Al}$, the minimum, first quartile, median and third quartile were the same (B). Postoperatively, the HHI, Al and HUV significantly improved (indicated with an asterisk; A, B and D). A Bonferroni correction was used and a $p$ value $<0.0125$ was considered statistically significant.

\section{Discussion}

The ABG, cVEMP thresholds and symptomatology in SCD patients have previously been studied. However, scant data exists regarding the relationship between objective and subjective outcome measures in SCD syndrome and existing studies are limited to case reports. We sought to fill this gap and evaluate the relationship between audiometric and vestibular testing and patient symptomatology in SCD. This is the largest study that 
investigates the relationship between objective and subjective outcome measures in SCD patients $(2,8,10,14,17)$.

We found that patients who reported hearing loss had larger $250 \mathrm{~Hz}$ ABGs than patients without subjective hearing loss. The presence of other auditory and vestibular symptoms, as well as the severity of symptoms as measured by the $\mathrm{HHI}, \mathrm{DHI}, \mathrm{Al}$ and SF-36, were not related to the magnitude of the ABG or cVEMP threshold (although the relationship between $A B G$ and $\mathrm{HHI}$ was near significant). Because we did not find a significant relationship between the degree of $A B G$ and cVEMP threshold abnormality and symptoms (with the exception of $250 \mathrm{~Hz} A B G s$ and the presence of subjective hearing loss) we wondered if perhaps a threshold effect would be present, i.e. once a threshold of abnormality is exceeded there is a higher likelihood of symptoms. To assess if this threshold effect was present, we performed a threshold-effect analysis in which we arbitrarily separated the ABG and cVEMP data into "normal" and "abnormal" groups and performed a Fisher's exact test. The presence of an ABG of $15 \mathrm{~dB} \mathrm{HL}$ or more and a cVEMP threshold of less than $98 \mathrm{~dB}$ peSPL was considered "abnormal". These strict cutoff values segregated patients with clear ABGs and very low CVEMP thresholds from patients with ABGs and CVEMP thresholds that are closer to normal. Similar to our previous analysis, we did not find a significant association between "normal" and "abnormal" ABG or cVEMP thresholds and the presence of symptoms, except for subjective hearing loss and abnormal ABG $(p=0.001)$.

The lack of correlation between vestibular symptoms and objective findings is not a new phenomenon. Mehta et al. (8) investigated the relationship between true dehiscence and thin bone (47 versus 17 ears) on CT imaging and found significantly lower thresholds in the former group; nevertheless, they found no significant difference in $\mathrm{DHI}$ scores between groups. More broadly, a lack of a correlation between subjective and objective findings has also been described for other diseases. Keim (32) found that only $46 \%$ of patients with unilateral labyrinthine impairment complained of vertigo. Jacobson and McCaslin (33) found no significant difference in DHI between patients with and without compensated unilateral peripheral vestibular systems after acute unilateral peripheral vestibular dysfunction based on ENG and rotary chair tests. A similar lack of correlation between subjective and objective measures has been observed in patients with tinnitus and hearing loss $(34,35)$. A possible explanation for these findings could be that the selected outcome measures were heterogeneous and not sufficiently precise $(31,36)$. For example, individual symptoms were appreciated in a binary fashion (present versus absent) that might obscure a correlation. Furthermore, perceptions of and coping with symptoms varies widely with each patient and can be influenced by biopsychosocial factors other than the underlying disease process (37).

We found that closure of the $250 \mathrm{~Hz}$ ABG following SCD repair arose from a combination of an increase in bone-conduction threshold and a decrease in airconduction threshold. This finding agrees with previous studies and suggests that preoperative bone-conduction thresholds were enhanced by the dehiscence. It also 
suggests that surgery normalizes this pseudo-conductive hearing loss (19,38-40). We also found an increase in postoperative CVEMP threshold that is consistent with previous studies $(17,38)$. Together, these findings indicate that both ABG and CVEMP threshold are useful in evaluating the effect of surgical repair. The closure of the ABG and increase in CVEMP threshold suggest a decrease in sound-energy flow towards the dehiscence after third window closure, consistent with a successful repair. Preoperatively, the $250 \mathrm{~Hz} A B G$ and CVEMP threshold have proven their value in the diagnosis of SCD, especially when using our combined Third Window Indicator (TWI), which is calculated by subtracting the $250 \mathrm{~Hz} A B G$ from the cVEMP threshold (16). To this end, although $A B G$ and CVEMP may not correlate with specific audiovestibular symptoms, they are invaluable tools to aid in preoperative diagnosis and provide a more objective measure of a physiologically effective and durable repair.

Although audiometry and CVEMP testing are useful in the diagnosis of SCD, the decision to surgically repair a dehiscence should be made based on a patient's subjective symptomatology and functional impairment. Improvement in Al after surgery is a consistent finding in literature $(20,24,41)$. In contrast, improvement in $\mathrm{HHI}$ is inconsistent (20). Likewise, improvement in $\mathrm{DHI}$ is variable, with some studies showing improvement, some showing improvement only in highly disabled patients, and others showing no significant improvement $(20,42,43)$.

Regarding $\mathrm{DHI}$ scores, it has previously been suggested that female gender and presence of migraine may be associated with a higher DHI score after surgery (41). Our cohort of eight patients (2M, 6F; 3 patients with migraine) with available pre- and postoperative $\mathrm{DHI}$ scores was too small to statistically evaluate the effect of gender and migraine. However, an observation of the data did not indicate an effect of gender or migraine, with an average $\mathrm{DHI}$ decrease of 33.3 points (SD: 28.6) in women and a decrease of 2 points in one male and an increase of 64 points in the other male. The three migraineurs showed a decrease in their DHI score of 32, 40 and 64 points, while among nonmigraineurs three patients had a decrease of 2, 16 and 48 points and two patients had an increase of 18 and 64 points.

Our findings of improvement in $\mathrm{HHI}$ and $\mathrm{Al}$ after surgery indicate that patients with auditory symptoms can benefit from surgical dehiscence repair. Postoperative improvement appears less likely for those patients with vestibular symptoms, as this cohort showed no significant improvement in $\mathrm{DHI}$. It is also possible that the $\mathrm{DHI}$ is not sensitive enough to capture details about SCD specific symptoms and that vestibular symptoms change after surgery which would not result in an improvement of DHI. Improvement in auditory symptoms is expected because the energy-shunting mechanism that causes auditory symptoms is reversed by plugging the dehiscence. Relief of vestibular symptoms, however, likely involves a different mechanism. Specifically, plugging the superior semicircular canal reduces energy shunting and acoustical activation of the saccule, but it also interrupts normal endolymph movement in the superior semicircular canal. In this regard, patients who have had SCD plugging 
continue to have symptoms of imbalance to rotational movement in the superior canal plane, associated with reduced VOR gain (44). Patients should be counseled preoperatively that their auditory symptoms have a greater likelihood of improving compared to vestibular symptoms following surgical repair and their vestibular symptoms might change.

An ideal repair would be one that repairs the dehiscence and maintains the canal lumen and associated sensory neuroepithelium. Modern approaches to "resurface" SCD often represent a gentle form of plugging, and have been associated with a higher recurrence of symptoms compared to plugging $(45,46)$. Our findings underscore the importance of refining SCD repair techniques. For example, a three-dimensional (3D) printed prosthesis that approximates the density of otic capsule bone is one way to provide a "personalized" repair that is tailored to the defect and maintains the patency of the superior canal (47).

Limitations of this study include the relatively small number of SCD patients from whom we had complete questionnaire data perioperatively. Although patient-reported outcome measures are currently used at our institution for prospective data collection on symptoms before and after surgery, many patients did not have complete questionnaires available, and information about symptoms therefore had to be extracted from medical records. Unfortunately, the reporting of symptoms in the medical records was not always consistent or complete and the number of patients with available postoperative data was limited, which decreased the power of this study. This also limited our ability for a multifactorial analysis. The prospective use of questionnaires to assess patients with SCD at our institution is now consistently performed and will provide more information about symptomatology in SCD patients in the future. In addition, more sensitive outcome measures are needed to more accurately evaluate symptoms in patients with SCD syndrome (31).

\section{Conclusion}

While threshold audiometry and CVEMP are important tools in the diagnosis and postoperative evaluation of SCD patients, there does not seem to be a strong correlation between these objective measures and patients' subjective symptoms before and after surgical repair. 


\section{References}

1. Minor LB, Solomon D, Zinreich JS, et al. Sound- and/or pressure-induced vertigo due to bone dehiscence of the superior semicircular canal. Arch Otolaryngol Head Neck Surg 1998;124:249-58.

2. Niesten ME, Hamberg LM, Silverman JB, et al. Superior canal dehiscence length and location influences clinical presentation and audiometric and cervical vestibular-evoked myogenic potential testing. Audiol Neurootol 2014;19:97-105.

3. Yuen HW, Eikelboom RH, Atlas MD. Auditory manifestations of superior semicircular canal dehiscence. Otol Neurotol 2009;30:280-5.

4. Rosowski JJ, Songer JE, Nakajima HH, et al. Clinical, experimental, and theoretical investigations of the effect of superior semicircular canal dehiscence on hearing mechanisms. Otol Neurotol 2004;25:323-32.

5. Ho ML, Moonis G, Halpin CF, et al. Spectrum of third window abnormalities: semicircular canal dehiscence and beyond. AJNR Am J Neuroradiol 2017;38:2-9.

6. Rauch SD, Silveira MB, Zhou G, et al. Vestibular evoked myogenic potentials versus vestibular test battery in patients with Meniere's disease. Otol Neurotol 2004;25:981-6.

7. Rauch SD, Zhou G, Kujawa SG, et al. Vestibular evoked myogenic potentials show altered tuning in patients with Meniere's disease. Otol Neurotol 2004; 25:333-8.

8. Mehta R, Klumpp ML, Spear SA, et al. Subjective and objective findings in patients with true dehiscence versus thin bone over the superior semicircular canal. Otol Neurotol 2015;36:289-94.

9. Hunter JB, Patel NS, O'Connell BP, et al. Cervical and ocular VEMP testing in diagnosing superior semicircular canal dehiscence. Otolaryngol Head Neck Surg 2017;156:917-23.

10. Benamira LZ, Alzahrani M, Saliba I. Superior canal dehiscence: can we predict the diagnosis? Otol Neurotol 2014;35:338-43.

11. Colebatch JG, Halmagyi GM, Skuse NF. Myogenic potentials generated by a click-evoked vestibulocollic reflex. J Neurol Neurosurg Psychiatry 1994;57:190-7.

12. Milojcic R, Guinan JJ Jr, Rauch SD, et al. Vestibular evoked myogenic potentials in patients with superior semicircular canal dehiscence. Otol Neurotol 2013; 34:360-7.

13. Govender S, Fernando T, Dennis DL, et al. Properties of $500 \mathrm{~Hz}$ air- and bone-conducted vestibular evoked myogenic potentials (VEMPs) in superior canal dehiscence. Clin Neurophysiol 2016;127:252231.

14. Roditi RE, Eppsteiner RW, Sauter TB, et al. Cervical vestibular evoked myogenic potentials (cVEMPs) in patients with superior canal dehiscence syndrome (SCDS). Otolaryngol Head Neck Surg 2009;141: 24-8.

15. Brantberg K. Vestibular evoked myogenic potentials (VEMPs): usefulness in clinical neurotology. Semin Neurol 2009;29:541-7.

16. Noij KS, Duarte MJ, Wong K, et al. Toward optimizing cervical vestibular evoked myogenic potentials (cVEMP): Combining air-bone gap and cVEMP thresholds to improve diagnosis of superior canal dehiscence. Otol Neurotol 2018;39:212-20.

17. Niesten ME, McKenna MJ, Herrmann BS, et al. Utility of cVEMPs in bilateral superior canal dehiscence syndrome. Laryngoscope 2013;123:226-32.

18. Welgampola MS, Myrie OA, Minor LB, et al. Vestibular-evoked myogenic potential thresholds normalize on plugging superior canal dehiscence. Neurology 2008;70:464-72.

19. Niesten ME, Stegeman I, Lookabaugh S, et al. Systematic review of outcomes following superior canal dehiscence surgery: determining best surgical candidates. In: Niesten ME. Evaluation and Management of Superior Canal Dehiscence syndrome. 2014;152-72.

20. Remenschneider AK, Owoc M, Kozin ED, et al. Health utility improves after surgery for superior canal dehiscence syndrome. Otol Neurotol 2015;36:1695-701.

21. Ziylan F, Kinaci A, Beynon AJ, et al. A comparison of surgical treatments for superior semicircular canal dehiscence: A systematic review. Otol Neurotol 2017;38:1-10.

22. Ventry IM, Weinstein BE. The hearing handicap inventory for the elderly: a new tool. Ear Hear 1982; 3:128-34.

23. Jacobson GP, Newman CW. The development of the Dizziness Handicap Inventory. Arch Otolaryngol Head Neck Surg 1990;116:424-7. 
24. Crane BT, Lin FR, Minor LB, et al. Improvement in autophony symptoms after superior canal dehiscence repair. Otol Neurotol 2010;31:140-6.

25. Ware JE Jr, Sherbourne CD. The MOS 36-item short-form health survey (SF-36). I. Conceptual framework and item selection. Med Care 1992;30:473-83.

26. Standards AN. ANSI S3.21-2004 Methods for Manual Pure-Tone Threshold Audiometry. New York, NY: Acoustical Society of America, 2004b.

27. Standards AN. ANSI S3.6-2004 Specification for Audiometers. New York, NY: Acoustical Society of America, 2004a.

28. Rosengren SM, Govender S, Colebatch JG. The relative effectiveness of different stimulus waveforms in evoking VEMPs: significance of stimulus energy and frequency. $J$ Vestib Res 2009;19:33-40.

29. Brazier J, Roberts J, Deverill M. The estimation of a preference-based measure of health from the SF36. J Health Econ 2002;21:271-92.

30. Ward BK, Wenzel A, Ritzl EK, et al. Near-dehiscence: clinical findings in patients with thin bone over the superior semicircular canal. Otol Neurotol 2013;34:1421-8.

31. Naert L, van de Berg R, van de Heyning $P$, et al. Aggregating the symptoms of superior semicircular canal dehiscence syndrome. Laryngoscope 2017 (in press).

32. Keim RJ. Comparison of symptoms and laboratory findings in unilateral vestibular dysfunction. Otolaryngol Head Neck Surg 1984;92:577-9.

33. Jacobson GP, McCaslin DL. Agreement between functional and electrophysiologic measures in patients with unilateral peripheral vestibular system impairment. J Am Acad Audiol 2003;14:231-8.

34. Ward LM, Baumann M. Measuring tinnitus loudness using constrained psychophysical scaling. Am J Audiol 2009;18:119-28.

35. Newman CW, Weinstein BE, Jacobson GP, et al. The hearing handicap inventory for adults: psychometric adequacy and audiometric correlates. Ear Hear 1990;11:430-3.

36. Ossen ME, Stokroos R, Kingma H, et al. Heterogeneity in reported outcome measures after surgery in superior canal dehiscence syndrome - a systematic literature review. Front Neurol 2017;8:1-21.

37. Langguth B, Kleinjung T, Fischer B, et al. Tinnitus severity, depression, and the big five personality traits. Prog Brain Res 2007;166:221-5.

38. Thomeer $\mathrm{H}$, Bonnard D, Castetbon V, et al. Long-term results of middle fossa plugging of superior semicircular canal dehiscences: clinically and instrumentally demonstrated efficiency in a retrospective series of 16 ears. Eur Arch Otorhinolaryngol 2016;273: 1689-96.

39. Ward BK, Agrawal $Y$, Nguyen E, et al. Hearing outcomes after surgical plugging of the superior semicircular canal by a middle cranial fossa approach. Otol Neurotol 2012;33:1386-91.

40. Van Haesendonck G, van de Heyning P, van Rompaey V. Retrospective cohort study on hearing outcome after transmastoid plugging in superior semicircular canal dehiscence syndrome: Our Experience. Clin Otolaryngol 2016;41:601-6.

41. Jung DH, Lookabaugh SA, Owoc MS, et al. Dizziness is more prevalent than autophony among patients who have undergone repair of superior canal dehiscence. Otol Neurotol 2015;36: 126-32.

42. Crane BT, Minor LB, Carey JP. Superior canal dehiscence plugging reduces dizziness handicap. Laryngoscope 2008;118:1809-13.

43. Bogle JM, Lundy LB, Zapala DA, et al. Dizziness handicap after cartilage cap occlusion for superior semicircular canal dehiscence. Otol Neurotol 2013;34:135-40.

44. Mantokoudis G, Saber Tehrani AS, Wong AL, et al. Adaptation and compensation of vestibular responses following superior canal dehiscence Surgery. Otol Neurotol 2016;37:1399-405.

45. Rodgers B, Lin J, Staecker H. Transmastoid resurfacing versus middle fossa plugging for repair of superior canal dehiscence: Comparison of techniques from a retrospective cohort. World J Otorhinolaryngol Head Neck Surg 20164;2:161-167.

46. Minor LB. Clinical manifestations of superior semicircular canal dehiscence. Laryngoscope 2005;115: 1717-27.

47. Kozin ED, Remenschneider AK, Cheng S, et al. Three-Dimensional Printed Prosthesis for Repair of Superior Canal Dehiscence. Otolaryngol Head Neck Surg 2015;153:616-9. 



\section{Chapter}

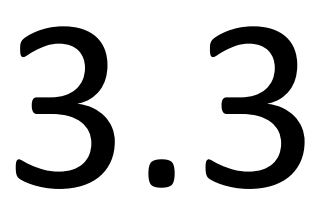

$2000 \mathrm{~Hz}$ tone bursts improve the detection of superior semicircular canal dehiscence syndrome 


\section{Abstract}

\section{Background}

The cervical vestibular evoked myogenic potential (cVEMP) test measures saccular and inferior vestibular nerve function. The cVEMP can be elicited with different frequency stimuli and interpreted using a variety of metrics. Patients with superior semicircular canal dehiscence syndrome (SCD) generally have lower cVEMP thresholds and larger amplitudes, although there is overlap with healthy subjects. The aim of this study was to evaluate which metric and frequency best differentiate healthy ears from SCD ears using cVEMP.

\section{Methods}

Twenty-one patients with SCD and 23 age-matched controls were prospectively included and underwent cVEMP testing at 500, 750, 1000 and $2000 \mathrm{~Hz}$. Sound level functions were obtained at all frequencies to acquire threshold and to calculate normalized peak-to-peak amplitude (VEMPn) and VEMP inhibition depth (VEMPid). Third Window Indicator (TWI) metrics were calculated by subtracting the $250 \mathrm{~Hz}$ airbone gap ( $A B G)$ from the ipsilateral cVEMP threshold at each frequency. Ears of SCD patients were divided into three groups based on CT imaging: dehiscent, thin or unaffected. The ears of healthy age-matched control subjects constituted a fourth group.

\section{Results}

Comparing metrics at all frequencies revealed that $2000 \mathrm{~Hz}$ stimuli were most effective in differentiating SCD from normal ears. ROC analysis indicated that for both $2000 \mathrm{~Hz}$ cVEMP threshold and for $2000 \mathrm{~Hz}$ TWI, 100\% specificity could be achieved with a sensitivity of $92.0 \%$. With $2000 \mathrm{~Hz}$ VEMPn and VEMPid at the highest sound level, $100 \%$ specificity could be achieved with a sensitivity of $96.0 \%$.

\section{Conclusion}

The best diagnostic accuracy of cVEMP in SCD patients can be achieved with $2000 \mathrm{~Hz}$ tone burst stimuli, regardless of which metric is used. 


\section{Introduction}

Patients with semicircular canal dehiscence syndrome (SCD) suffer from a variety of auditory and vestibular symptoms caused by a bony defect in the superior semicircular canal (SSC) that creates a "third window" (1). Acoustic stimulation of an ear with a "third window" causes energy from stapes footplate motion to shunt towards the dehiscence and away from the cochlear partition. As a result, the pressure difference across the basilar membrane decreases, while energy transmission to the vestibular sense organs increases $(2,3)$. The diagnosis of SCD is generally based on a combination of tests including symptomatology, threshold audiometry and immitance testing, vestibular evoked myogenic potential (VEMP) testing and temporal bone CT imaging (2). These tests can be time consuming and costly.

The cervical vestibular evoked myogenic potential (cVEMP) test measures saccular and inferior vestibular nerve function. When the saccule is acoustically or mechanically stimulated, vestibulocollic projections inhibit the (mostly) ipsilateral sternocleidomastoid muscle (SCM) which can be measured with electromyography (EMG) (4). An SCD third window can lead to low thresholds and large amplitudes on cVEMP testing compared to healthy controls (4-11). Multiple metrics can be used to assess the CVEMP, including the normalized peak-to-peak amplitude (VEMPn), cVEMP threshold, the third window indicator (TWI) (12) and a new metric called VEMP inhibition depth (VEMPid), which estimates the percentage reduction in spike rate of the SCM motoneurons during CVEMP testing and has not previously been used in patients (13).

Previous studies indicated that threshold was more valuable than peak-to-peak amplitude in distinguishing healthy from pathologic ears in both SCD and Menière's disease $(7,14,15)$. Thresholds, however, overlap between the SCD and healthy populations, and threshold measurement can be time consuming. Although the CVEMP is not a painful test, longer testing time can result in neck muscle discomfort. A cVEMPbased diagnostic tool for SCD patients that seems to be more useful than CVEMP threshold alone is the Third Window Indicator (TWI) (12). The TWI combines CVEMP threshold with the audiometric low-frequency air-bone gap ( $A B G)$ from the ipsilateral ear and improves the differentiation of SCD patients from healthy subjects (12). The TWI was developed using a retrospective analysis of data and has not been compared to other metrics prospectively.

Although many institutions record CVEMP only with $500 \mathrm{~Hz}$ tone bursts, testing at multiple frequencies appears to be a valuable tool in differentiating patients with Menière's disease (14). In our institution, clinical cVEMP testing includes stimulation with 500, 750 and $1000 \mathrm{~Hz}$ tone bursts. In SCD patients, cVEMP amplitude and threshold tuning curves broaden (15). A similar broadening of tuning has been found in ocular VEMPs (oVEMP) (16). Manzari et al. used high frequency stimuli up to $8000 \mathrm{~Hz}$ to evoke oVEMP responses and concluded that $4000 \mathrm{~Hz}$ was the optimal frequency to 
distinguish dehiscent from healthy ears (16). These CVEMP and oVEMP findings suggest that stimulation of SCD patients with higher frequencies may be of clinical value. Since cVEMPs above $1000 \mathrm{~Hz}$ were not available in the clinical retrospective dataset, TWI has not previously been evaluated at higher frequencies.

The current study was designed to prospectively test SCD patients and systematically evaluate the same sound levels, frequencies and metrics in every subject, something that could not be done in our retrospective study (12). $2000 \mathrm{~Hz}$ was included to evaluate cVEMP metrics at a higher frequency. Although Manzari et al. concluded that $4000 \mathrm{~Hz}$ was optimum for oVEMP measurements, we decided not to add another frequency to our protocol because this would increase testing time and because intense $4000 \mathrm{~Hz}$ tones can be very uncomfortable. The aim of this prospective study was to determine which CVEMP metric and which stimulus frequency best identify SCD patients to optimize the applicability of CVEMP as a diagnostic tool in SCD patients.

\section{Methods}

\section{Subjects}

Between April 2016 and November 2017, twenty-one patients with symptomatic and radiographically confirmed SCD and 23 age-matched healthy controls were prospectively included in this study. Since middle ear pathology can influence CVEMP outcomes (decrease amplitude and increase thresholds), patients and control subjects with middle ear pathology were excluded. Middle ear pathology was identified by a combination of exam findings, including threshold and immitance audiometry. Those with an $A B G>10 \mathrm{~dB}$ at any tested frequency were further evaluated with immittance audiometry. Ears with an $A B G>10 \mathrm{~dB}$ in combination with abnormal tympanograms and/or absent reflexes were excluded from this study. For the healthy controls, additional exclusion criteria were a history of hearing loss, vertigo, balance problems, neurological disorders and musculoskeletal disease. This study was approved by the Human Studies Committee of the Massachusetts Eye and Ear Infirmary (protocol No. 13-097H, principal investigator S.D. Rauch).

\section{Radiology}

Eleven patients underwent CT imaging at our institution and ten patients had CT imaging from an outside hospital. For 7 patients, a cone beam CT scanner (3D Accuitomo 170; J. Morita, Irvine, CA, USA) was used generating images with a slice thickness of $0.5 \mathrm{~mm}$ and an axial pixel dimension of $0.125 \mathrm{~mm}$. Multidetector row CT scanners were used for all other patients, generating images with an average slice thickness of $0.70 \mathrm{~mm}(0.5-1.25 \mathrm{~mm})$. All superior semicircular canals were evaluated by 
a neuroradiologist at our institution using reformatted images in Stenvers and Pöschl planes created with multiplanar reconstruction. Stenvers plane images were perpendicular and Pöschl images were parallel to the plane of the superior semicircular canal. The neuroradiologist evaluating these images subjectively determined whether the semicircular canals were dehiscent, had a thin layer of bone covering the canal, or were normal in appearance. Based on the radiologist's interpretation, the results from each side of each subject were divided into three groups: dehiscent, thin bone, or unaffected ("unaffected" denotes a normal bone-covered superior canal contralateral to an SCD ear).

\section{Audiometry}

Air- and bone-conduction tonal thresholds were measured for all subjects at octave frequencies from 250 to $4000 \mathrm{~Hz}$. If the difference between air- and unmasked boneconduction thresholds was larger than $10 \mathrm{~dB} \mathrm{HL}$, bone-conduction thresholds were masked. The ABG was calculated at each tested frequency by subtracting the boneconduction threshold from the air-conduction threshold. Ears with either an ABG of $\leq 10 \mathrm{~dB}$ at any frequency or an $\mathrm{ABG}>10 \mathrm{~dB}$ in combination with normal tympanograms and present acoustic reflexes were considered to have normal middle ear function and were included in this study.

\section{Cervical Vestibular Evoked Myogenic Potentials}

cVEMPs were obtained with subjects sitting up straight and the head turned away from the stimulated ear to elicit contraction of the ipsilateral SCM. Electromyographic (EMG) activity was recorded from four surface electrodes: a positive electrode on the middle belly of each SCM, a reference electrode at the midpoint between SCM attachments to the sternum, and a ground electrode on the midline forehead. Ipsilateral SCM EMG amplitude was monitored and subjects contracted their SCM to produce $>45 \mu \mathrm{V}$ root mean square ( $\mathrm{rms}$ ). All subjects were able to maintain SCM contractions above this limit and the few waveforms $<45 \mu \mathrm{V}$ rms were not included. EMG activity was amplified, bandpass filtered and sampled at $50 \mathrm{kHz}$ with a 16-bit analog-to-digital converter (National Instruments).

cVEMP were obtained using 500, 750, 1000 and $2000 \mathrm{~Hz}$ tone bursts generated by custom-programmed evoked potential software (National Instruments 16-bit digital I/O board) using a Blackman gating function with two cycle $(4.0 \mathrm{~ms}$ at $500 \mathrm{~Hz}, 2.5 \mathrm{~ms}$ at $750 \mathrm{~Hz}, 2 \mathrm{~ms}$ at $1000 \mathrm{~Hz}, 1 \mathrm{~ms}$ at $2000 \mathrm{~Hz}$ ) rise and fall times and no plateau. Tone bursts were presented monaurally via circumaural headphones (Telephonics TDH-49) at a repetition rate of 13 bursts/s; cVEMP responses averaged between 200 to 300 single sweeps. In the SCD group, tone bursts were presented at 83, 93, 103 and $123 \mathrm{~dB}$ peak sound pressure level (peSPL), while in the healthy control group, tone bursts were presented at 93,103, 113 and $123 \mathrm{~dB}$ peSPL (123 dB peSPL is equivalent to $90 \mathrm{~dB} \mathrm{nHL}$ ). 
SCD patients generally have lower thresholds and the protocol with a large sound-level step was chosen to reduce testing time. Presentation orders for sound level, frequency and side were randomized.

\section{VEMP metrics}

The collection of sound level functions at all frequencies allowed for the calculation of VEMPn (normalized peak-to-peak amplitude) and VEMPid (VEMP inhibition depth) at every sound level and for determining threshold. The CVEMP waveform was normalized using trace-by-trace normalization (17). Trace-by-trace normalization divides each raw EMG trace by the rms value of that trace's EMG (the trace-EMG includes all $77 \mathrm{~ms}$ of the time from one stimulus to the next). VEMPn was obtained by measuring the amplitude difference between P1 and N1 of the average normalized waveform.

VEMPid is a template correlation method that estimates the percentage reduction in spike-rate of the SCM motoneurons that is elicited by acoustic stimulation of the saccule. In this study, VEMPid was calculated using a generic template created from cVEMP responses of healthy subjects $(13,18)$. Template correlation values $(T C V s)$ were calculated using the point-by-point correlation of each individual trace in a cVEMP response with the generic template. VEMPid was calculated by dividing the mean of all 200 to 300 TCVs by the standard deviation of the TCVs and multiplying by 0.2 . Although Prakash et al. originally used a subject-specific template, a generic template can be used as long as the generic template latency is adjusted to the patient's response $(13,18)$.

cVEMP threshold was determined after responses were measured for the 4 preset sound levels. Starting at the highest sound level for which no cVEMP response was observed, recordings were made by increasing the sound level in $5 \mathrm{~dB}$ steps until a response was observed. Threshold was defined as the lowest sound level at which a cVEMP was present, as subjectively determined by the tester assessing the shape, size and latency of the response. If no response was present at our equipment limit (133 dB peSPL), the threshold was considered to be $10 \mathrm{~dB}$ above this limit.

TWIs (Third Window Indicators) were calculated by subtracting the $250 \mathrm{~Hz}$ ABG from the ipsilateral cVEMP threshold at each frequency. For example: a subject with a cVEMP threshold of $103 \mathrm{~dB}$ peSPL at a given frequency and a $250 \mathrm{~Hz} \mathrm{ABG}$ of $15 \mathrm{~dB}$ has a TWI of $88 \mathrm{~dB}$ at this frequency (12).

\section{Data analysis}

An independent $t$ test was performed to compare age of the SCD and healthy control groups. Full factorial analyses of variance (ANOVA) were performed to examine differences in ABG and cVEMP metrics (VEMPn, VEMPid, cVEMP threshold and TWI) among the four groups (dehiscent, thin, unaffected and healthy control) and to evaluate the effect of stimulus frequency on the different cVEMP metrics. Group, 
frequency and sound level were considered fixed factors, while subject was considered a random factor. Post hoc pairwise comparisons using a Bonferroni adjustment for multiple comparisons were performed to compare groups and frequencies. Receiver operating characteristic (ROC) curves were used to compare the different metrics and frequencies in their ability to distinguish dehiscent from healthy-control superior semicircular canals.

\section{Results}

\section{Patient characteristics}

Twenty-one patients with a radiographically confirmed SCD on at least one side were included (11 female, mean age: 50.1 years; range: 35-67 years). Based on CT imaging, $25 / 42$ ears were categorized as dehiscent, 9/42 as thin and 8/42 as unaffected. One of the "unaffected" ears was excluded based on audiometry indicating a potential middle ear problem, leaving 7 unaffected ears for analysis. We included 23 age-matched controls (14 women, mean age: 51.8 years, range: 26-68 years). There was no significant difference in age between the SCD and control group $(p=0.619)$.

\section{Air-bone gap}

Air-bone gaps were calculated at $250 \mathrm{~Hz}$ for every group (Figure 3.3.1A). As expected, the dehiscent ears had significantly larger ABGs compared to thin $(p=0.001)$, unaffected $(p<0.001)$ or healthy-control ears $(p<0.001)$. No significant differences were found for any of the other combinations.
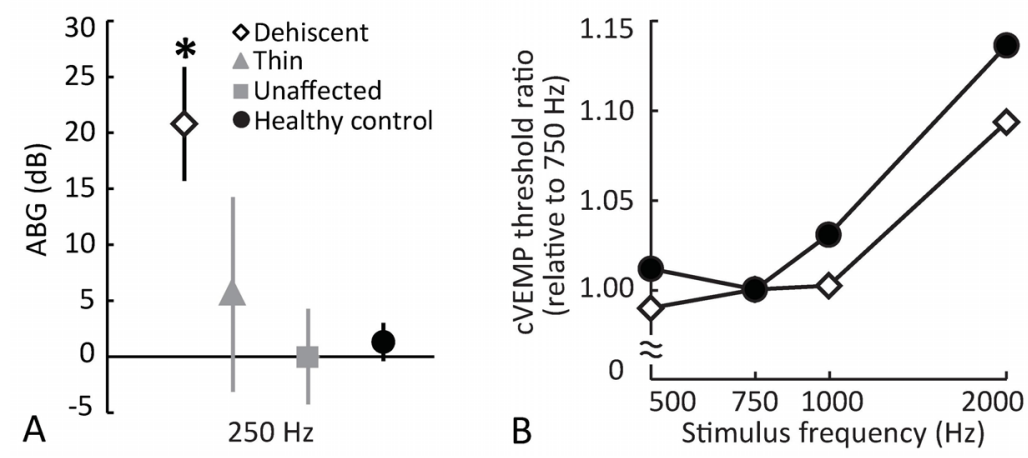

Figure 3.3.1 Average air-bone gap (ABG) at $250 \mathrm{~Hz}$ for each group (A). Error bars represent $95 \%$ confidence intervals. $A B G$ was significantly higher for the dehiscent group (asterisk) compared to thin, unaffected and healthy-controls. There were no significant differences among unaffected, thin and healthy-control ears. Average cVEMP threshold tuning relative to the $750 \mathrm{~Hz}$ threshold (B). As can be appreciated visually, the dehiscent-ears tuning curve is flatter than the healthy-ears tuning curve. 


\section{cVEMP}

cVEMP thresholds and TWIs were significantly lower for the dehiscent group compared to thin $(p<0.001)$, unaffected $(p<0.001)$ and healthy-control ears $(p<0.001)$, regardless of frequency (Figures 3.3.2A and 3.3.2B). No significant differences were found for any other combination. Regarding frequency, thresholds and TWIs were significantly higher in all four subject groups at $2000 \mathrm{~Hz}$ compared to $500(p<0.001)$, $750(p<0.001)$ and $1000 \mathrm{~Hz}(p<0.001)$. No significant differences were found for any other frequency combination. At $2000 \mathrm{~Hz}$, a response could not be elicited in 19/46 of the healthy control ears, while a response could always be elicited in the dehiscent ears.
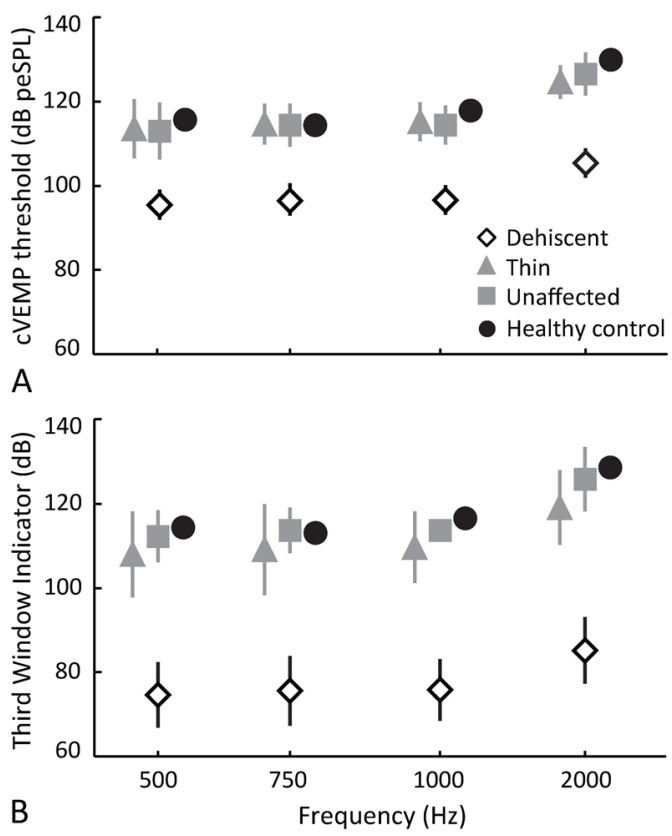

Figure 3.3.2 Average cVEMP threshold (A) and Third Window Indicator (TWI) (B) for 500, 750, 1000 and $2000 \mathrm{~Hz}$. Error bars represent the 95\% confidence intervals. Both cVEMP thresholds and the TWI were significantly lower in the dehiscent group compared to the thin, unaffected and healthy-control groups.

On average, $750 \mathrm{~Hz}$ yielded the lowest threshold in the healthy control ears (Figure 3.3.1B). To evaluate whether there were differences in frequency tuning between dehiscence patients and healthy controls, tuning curves were calculated using the $750 \mathrm{~Hz}$ threshold as the reference. As can be appreciated visually, the tuning curve for dehiscent ears is slightly flatter than the healthy-control tuning curve (Figure 3.3.1B). 
For both VEMPn and VEMPid, there was a significant interaction between frequency and stimulus level $(F=6.106, p<0.001$ and $F=8.771, p<0.001)$, with a larger effect of frequency at the highest sound levels (Figure 3.3.3). There was also a significant interaction between dehiscence groups and stimulus level for both VEMPn and VEMPid $(F=65.688, p<0.001$ and $F=78.942, p<0.001)$, with a larger effect of stimulus level in the dehiscent group compared to the other groups. As seen previously, VEMPid decreased to zero at the lower stimulus levels (i.e. when no response was present), while the VEMPn plateaued above zero (Figure 3.3.3) (19).

Because cVEMP outcomes are known to be affected by age, we used Pearson correlation coefficients to evaluate whether age influenced our cVEMP outcomes. Similar to findings by others, VEMPn significantly decreased with age for all frequencies in the healthy control group, while threshold significantly increased $(20,21)$. In the dehiscent group there was no significant effect of age, indicating that the presence of a dehiscence reduces the age effect (data not shown).

\section{ROC curves}

ROC curves were created to compare the ability of the four metrics (cVEMP threshold, TWI, VEMPn and VEMPid) and the four frequencies (500, 750, 1000 and $2000 \mathrm{~Hz}$ ) to distinguish dehiscent from the healthy ears. Based on the area under the curve (AUC), $2000 \mathrm{~Hz}$ was the best frequency for VEMP metrics to distinguish healthy from dehiscent ears (Figures 3.3.4 and 3.3.5). However, for the TWI, AUCs were slightly better at 500 and $1000 \mathrm{~Hz}$ (Figure 3.3.4).

We think that a more useful ROC measure is the sensitivity when there is $100 \%$ specificity. Using sensitivity of the $123 \mathrm{~dB}$ peSPL data, $2000 \mathrm{~Hz}$ was the best frequency for every metric to distinguish healthy from dehiscent ears (Figures 3.3.4 and 3.3.5). For cVEMP threshold and TWI at $2000 \mathrm{~Hz}$, a specificity of $100 \%$ could be reached with a sensitivity of $92 \%$ (Table 3.3.1, Figure 3.3.4) for cutoffs of 118 and $108 \mathrm{~dB}$ peSPL, respectively. These values correspond to a positive predictive value of $100 \%$ and a negative predictive value of $95.8 \%$, meaning that all patients with a positive test (cVEMP threshold $<118 \mathrm{~dB}$ peSPL or TWI $<108 \mathrm{~dB}$ ) have a dehiscent SCC on CT (Table 3.3.1, Figure 3.3.4). A higher sensitivity of $96 \%$, in combination with a $100 \%$ specificity, can be reached for both VEMPn and VEMPid at $2000 \mathrm{~Hz}$ and $123 \mathrm{~dB}$ peSPL (Table 3.3.1, Figure 3.3.5) for cutoff values of 0.67 and $9.2 \%$ respectively. That is, all patients with a positive test (VEMPn $>0.67$ and VEMPid $>9.2 \%$ ) have a dehiscent SCC on CT (Table 3.3.1, Figure 3.3.5). An exact McNemar's test evaluated the difference in sensitivity (92 versus $96 \%$ ) for the different metrics and revealed no statistically significant difference $(p=1.000)$. 


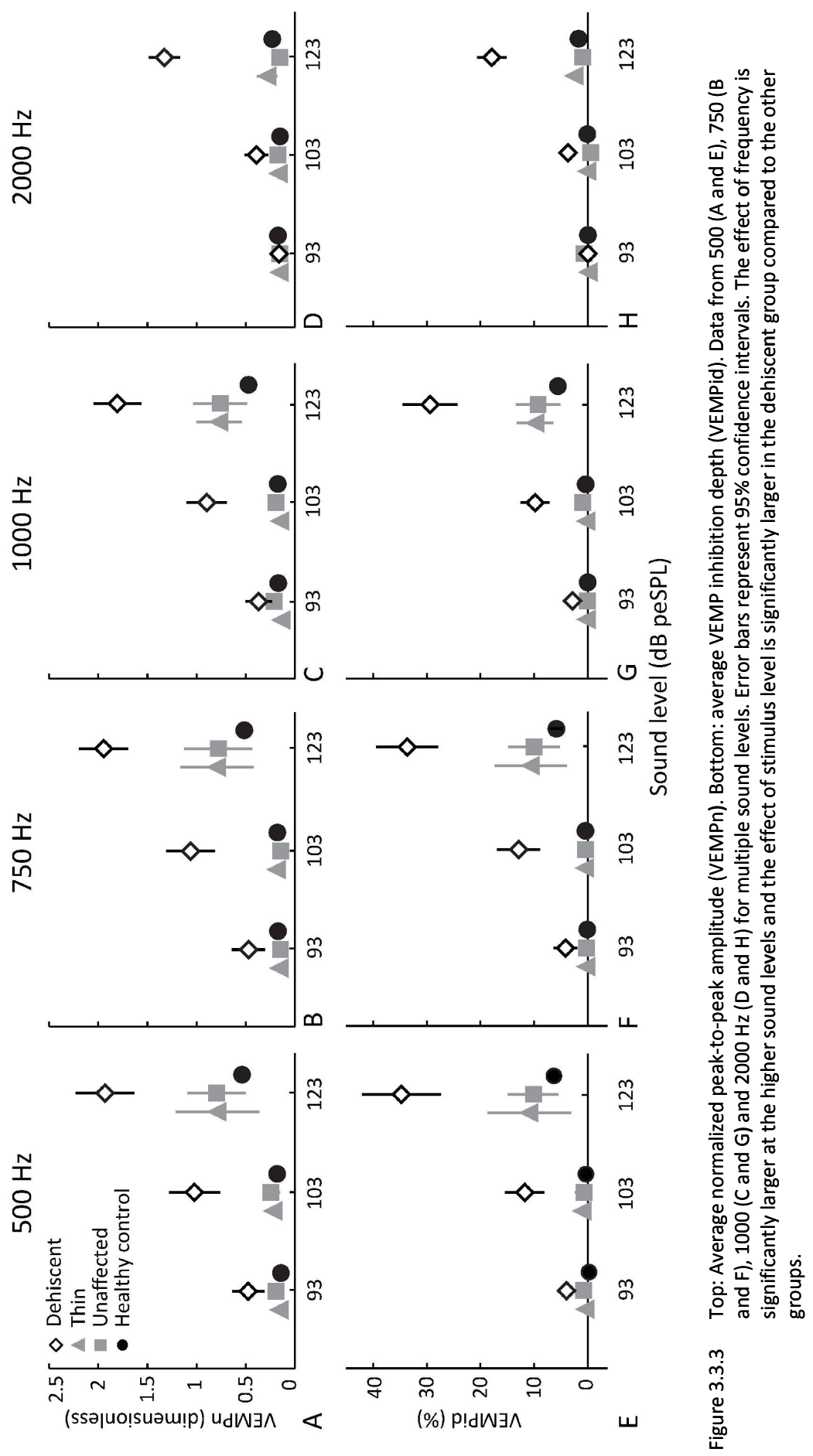




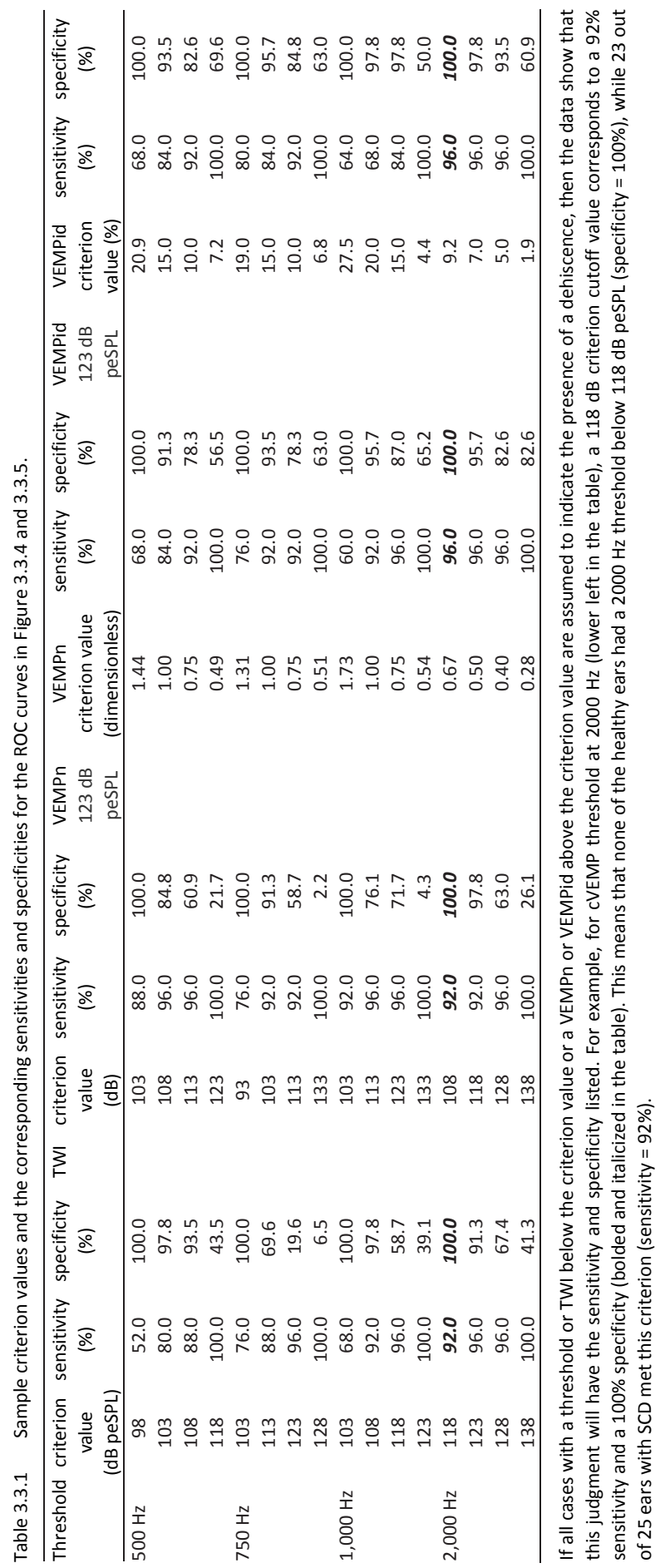



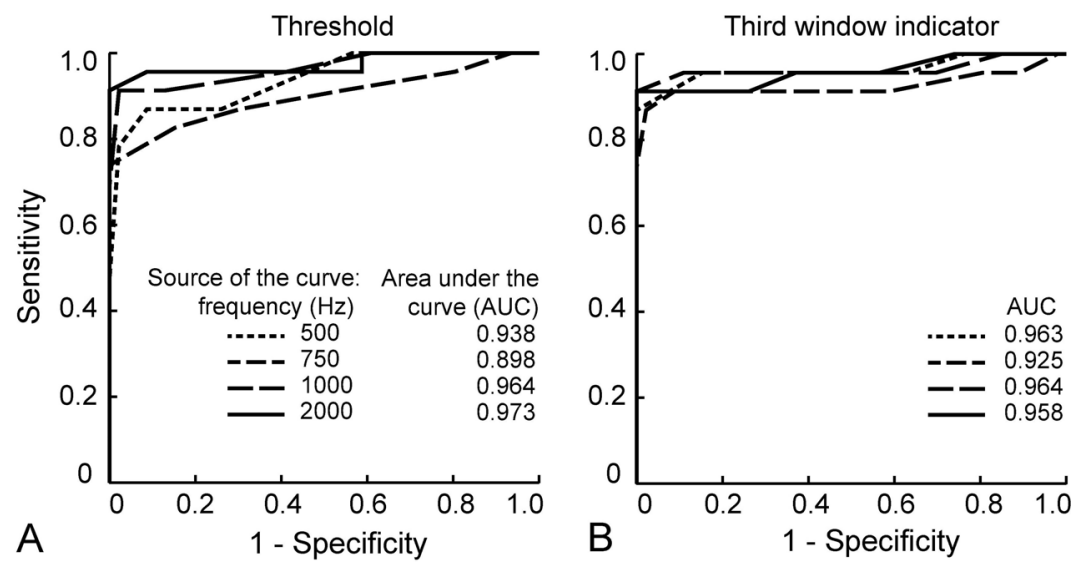

Figure 3.3.4 Receiver operating characteristic (ROC) curves displaying sensitivity of detecting a dehiscence versus false positive rate ( 1 - specificity) for cVEMP thresholds (A) and Third Window Indicators (TWIs) (B) for 500, 750, 1000 and $2000 \mathrm{~Hz}$. Insets show the frequency key and the corresponding area under the curve (AUC). cVEMP threshold and TWI data have $5 \mathrm{~dB}$ steps so that normal and affected subjects can have the same threshold. Thus, a change in cutoff value can produce a change in both the sensitivity and specificity at once, which causes points to be connected by diagonal lines.

\section{Discussion}

This study investigated which cVEMP metric and frequency were best at distinguishing healthy from dehiscent semicircular canals. At the highest sound level used for direct comparisons (123 dB peSPL) and for every metric used, $2000 \mathrm{~Hz}$ produced the highest sensitivity in detecting a dehiscence with $100 \%$ specificity. Sensitivities of $\geq 92 \%$ could be reached for every metric and VEMPn and VEMPid reached the highest sensitivity (96.0\%). Although $2000 \mathrm{~Hz}$ is not the frequency providing the largest amplitudes or lowest thresholds, the $2000 \mathrm{~Hz}, 123 \mathrm{~dB}$ peSPL stimuli provided the best separation between healthy and dehiscent ears. This was partly because healthy ears often did not have robust cVEMP responses at $2000 \mathrm{~Hz}$, while dehiscent ears did. At $2000 \mathrm{~Hz}$, VEMPn and VEMPid grow more with sound level in dehiscent ears than in patients with normal or thin bony covering of the SSC (Figure 3.3.3). 

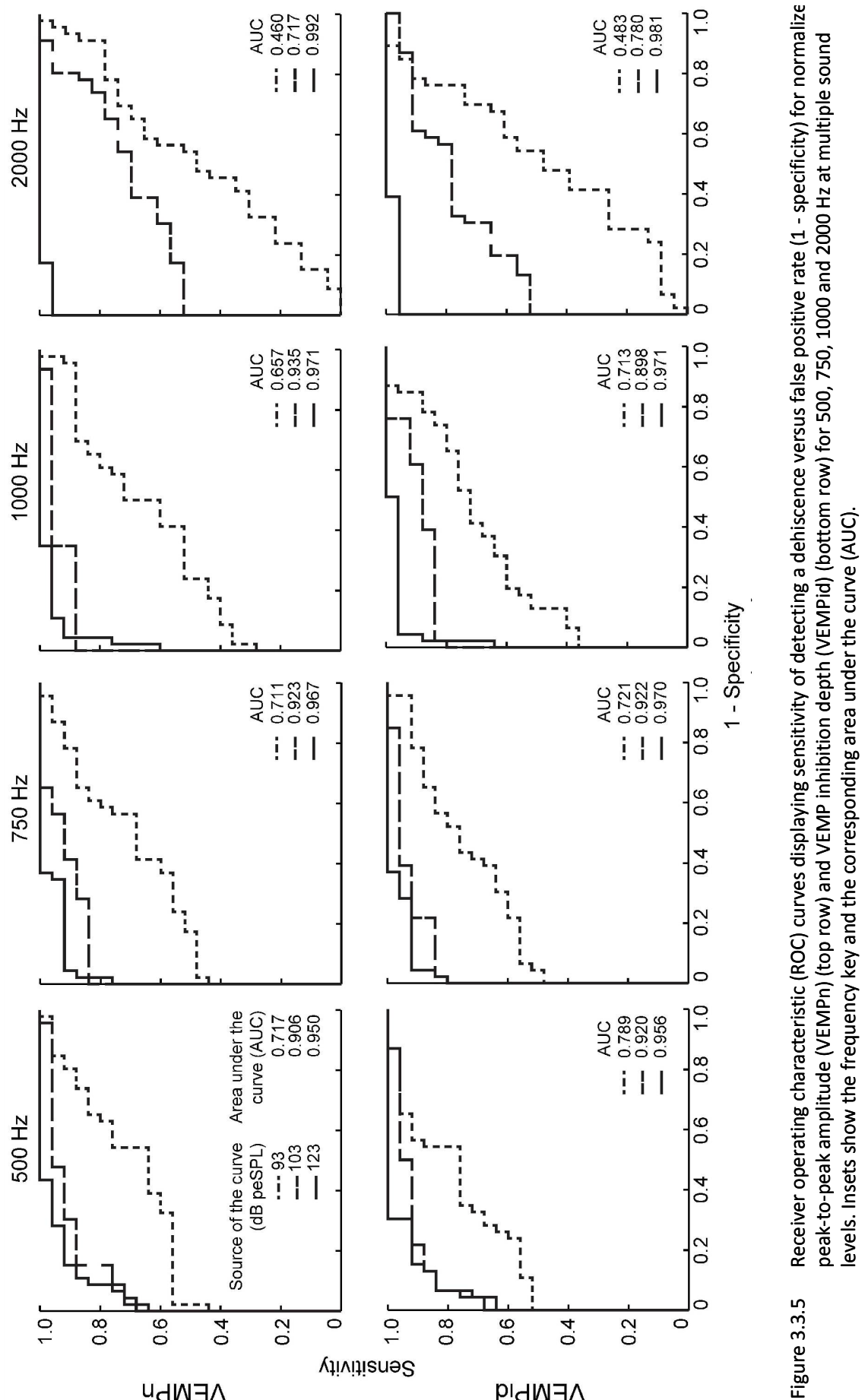

udWヨ^

p!dWヨ^

חִ 
At $103 \mathrm{~dB}$ peSPL, VEMPn and VEMPid at 500, 750 and $1000 \mathrm{~Hz}$ were better than 2000 $\mathrm{Hz}$ at separating dehiscent and healthy semicircular canals (Figure 3.3.5). The $2000 \mathrm{~Hz}$ data may do more poorly at levels below $123 \mathrm{~dB}$ peSPL because of differences in threshold across frequencies. Thresholds at $2000 \mathrm{~Hz}$ were higher for all subject groups (Figure 3.3.2) and $103 \mathrm{~dB}$ peSPL was not always sufficient to elicit a response at 2000 $\mathrm{Hz}$. At $103 \mathrm{~dB}$ peSPL, 750 and $1000 \mathrm{~Hz}$ VEMPn and VEMPid provided good sensitivities ( $80 \%$ and $88 \%$ ) with $100 \%$ specificity (Figure 3.3 .5 ) and comparable sensitivities were obtained with the TWI. Therefore, it could be argued that to reduce noise exposure the use of lower frequencies and lower sound levels is a reasonable alternative to a $2000 \mathrm{~Hz}, 123 \mathrm{~dB}$ peSPL stimulus.

This study investigated which CVEMP metric and frequency were best at distinguishing healthy from dehiscent semicircular canals. At the highest sound level used for direct comparisons (123 dB peSPL) and for every metric used, $2000 \mathrm{~Hz}$ produced the highest sensitivity in detecting a dehiscence with $100 \%$ specificity. Sensitivities of $\geq 92 \%$ could be reached for every metric and VEMPn and VEMPid reached the highest sensitivity (96.0\%). Although $2000 \mathrm{~Hz}$ is not the frequency providing the largest amplitudes or lowest thresholds, the $2000 \mathrm{~Hz}, 123 \mathrm{~dB}$ peSPL stimuli provided the best separation between healthy and dehiscent ears. This was partly because healthy ears often did not have robust cVEMP responses at $2000 \mathrm{~Hz}$, while dehiscent ears did. At $2000 \mathrm{~Hz}, \mathrm{VEMPn}$ and VEMPid grow more with sound level in dehiscent ears than in patients with normal or thin bony covering of the SSC (Figure 3.3.3).

At $103 \mathrm{~dB}$ peSPL, VEMPn and VEMPid at 500, 750 and $1000 \mathrm{~Hz}$ were better than 2000 $\mathrm{Hz}$ at separating dehiscent and healthy semicircular canals (Figure 3.3.5). The $2000 \mathrm{~Hz}$ data may do more poorly at levels below $123 \mathrm{~dB}$ peSPL because of differences in threshold across frequencies. Thresholds at $2000 \mathrm{~Hz}$ were higher for all subject groups (Figure 3.3.2) and $103 \mathrm{~dB}$ peSPL was not always sufficient to elicit a response at 2000 $\mathrm{Hz}$. At $103 \mathrm{~dB}$ peSPL, 750 and $1000 \mathrm{~Hz}$ VEMPn and VEMPid provided good sensitivities ( $80 \%$ and $88 \%$ ) with $100 \%$ specificity (Figure 3.3 .5 ) and comparable sensitivities were obtained with the TWI. Therefore, it could be argued that to reduce noise exposure the use of lower frequencies and lower sound levels is a reasonable alternative to a $2000 \mathrm{~Hz}, 123 \mathrm{~dB}$ peSPL stimulus.

Compared to the standard multi-frequency testing in our clinic, a single measurement using a $123 \mathrm{~dB}$ peSPL $2000 \mathrm{~Hz}$ toneburst would reduce the total sound exposure. The $2000 \mathrm{~Hz}$ toneburst has 2 cycle $(1 \mathrm{~ms}$ ) rise and fall times without a plateau so that the $123 \mathrm{~dB}$ peSPL is reached only momentarily at the sound peak. The brevity of this stimulus reduces the risk for acoustic trauma.

Omitting a cVEMP stimulus level between 103 and $123 \mathrm{~dB}$ peSPL is a limitation of this study. Because we wanted to compare 4 frequencies, obtain threshold at every frequency and keep testing time within 1.5 hours, it was necessary to take a large step (123 to $103 \mathrm{~dB}$ peSPL) in the SCD group. It should be investigated whether the VEMPn 
and VEMPid separation of SCD and healthy ears at $2000 \mathrm{~Hz}$ is equally good at stimulus levels between 103 and $123 \mathrm{~dB}$ peSPL.

The use of VEMPn and VEMPid is more favorable than cVEMP threshold because they require only one recording at each frequency and are less time-consuming. We favor the use of VEMPid because it has a "meaningful zero" value, i.e. VEMPid averages zero when no response is present, whereas VEMPn has a floor value caused by the EMG noise (Figure 3.3.3). The "meaningful zero" makes VEMPid easier to interpret (19). This is the first reported clinical application of VEMPid. At present there is no commercial device that calculates VEMPid from cVEMP recordings. However, the required calculations are described in Prakash et al. (13) and Noij et al. (18) and no difference in the cVEMP measurement setup is required.

\section{Comparisons with previous studies}

Multiple studies have looked at the usefulness of cVEMP amplitude or threshold in SCD patients (22-25), and some have looked at both $(15,26,27)$. Fife et al. summarized the outcomes of these studies and concluded that a sensitivity of $100 \%$ in combination with a specificity of $93 \%$ could be reached using normalized cVEMP amplitude (28). Using cVEMP threshold gives sensitivities ranging from $86 \%-91 \%$, with specificities ranging from $90 \%-96 \%$ (28). There are some limitations in comparing these studies, including the differences in methods used to obtain the CVEMP (e.g. click vs. toneburst and differences in stimulus level) and the reporting of outcomes (sensitivity and specificity are not always reported). Our study systematically compares the use of 4 different metrics at 4 different frequencies at multiple sound levels using the same methods in every subject, allowing for a reliable comparison. We favor the use of a cutoff value with a $100 \%$ specificity to avoid false diagnosis of SCD in patients without this disorder. As can be appreciated in Table 3.3.1 (see threshold at $500 \mathrm{~Hz}$ ), a large drop in sensitivity could be observed if a specificity of $100 \%$ is given priority. This highlights the importance of using a $2000 \mathrm{~Hz}$ tone burst, because this frequency gives a high sensitivity ( $\geq 92 \%)$ in combination with a $100 \%$ specificity.

We found a slightly flatter tuning curve for dehiscent ears than for healthy-control ears (Figure 3.3.1B), which is similar to findings of Taylor et al. (15). Taylor et al. investigated cVEMP frequency tuning in SCD patients and found that CVEMPs in SCD ears tended to tune to lower frequencies and that cVEMP threshold at 500 and $2000 \mathrm{~Hz}$ were equally good at separating healthy from dehiscent ears (15). This last finding is in contrast with our findings. Although our AUCs from 500 and $2000 \mathrm{~Hz}$ do not show large differences for each metric (Figures 3.3.4 and 3.3.5), the point in the ROC curve that corresponds to the highest sensitivity with $100 \%$ specificity does differ and is higher at $2000 \mathrm{~Hz}$ for all metrics (Table 3.3.1; Figures 3.3.4 and 3.3.5). For the clinical value of the test, the sensitivity and specificity provide more useful information than the AUC. For example, 
AUCs for CVEMP thresholds at 500 and $2000 \mathrm{~Hz}$ were 0.938 and 0.973 respectively (Figure 3.3.4), while the sensitivity, with $100 \%$ specificity, was $52 \%$ for $500 \mathrm{~Hz}$ and $92 \%$ for $2000 \mathrm{~Hz}$, which is a clinically relevant difference (Table 3.3.1).

Manzari et al. tested high frequency stimuli for oVEMP measurements and found that $4000 \mathrm{~Hz}$ was the best frequency to separate healthy from dehiscent ears with $100 \%$ specificity and sensitivity (16). While a slightly higher sensitivity at $4000 \mathrm{~Hz}$ with cVEMP is a possibility, our decision to use $2000 \mathrm{~Hz}$ stimuli but not $4000 \mathrm{~Hz}$ was made based on the increased patient discomfort that would be caused by both the discomfort of the high frequency sound itself and the increased testing time by adding another frequency.

Although the present study included only 21 SCD patients (25 dehiscent ears), the sensitivity (with $100 \%$ specificity) of our $500 \mathrm{~Hz}$ TWI is comparable to the TWI sensitivity from our larger retrospective study of 140 SCD patients (88 versus $82 \%$; both with cutoff values of $103 \mathrm{~dB}$ ) (12). This indicates that our cohort seems representative of this patient population. In the current study, thin-group TWIs were slightly smaller than healthy-group TWIs (Figure 3.3.2), but the differences were not statistically significant. Such differences were not seen in the larger retrospective TWI study (12), which indicates that the differences in the present study probably originate from the smaller number of subjects. Based on results of this study, we recommend measuring CVEMP using a $2000 \mathrm{~Hz}$ tone burst stimulus in suspected SCD patients. The use of VEMPn or VEMPid at a high stimulus level allows for a short test since only one recording is necessary on each side, significantly decreasing testing time, patient discomfort and noise exposure.

\section{Conclusion}

For all cVEMP metrics used, the best diagnostic accuracy of cVEMP in SCD patients is achieved with $2000 \mathrm{~Hz}$ tone burst stimuli. We recommend the use of $2000 \mathrm{~Hz}$ in clinical cVEMP testing of patients in whom SCD is suspected. 


\section{References}

1. Minor LB, Solomon D, Zinreich JS, et al. Sound- and/or pressure-induced vertigo due to bone dehiscence of the superior semicircular canal. Arch Otolaryngol Head Neck Surg 1998;124:249-58.

2. Ho ML, Moonis G, Halpin CF, et al. Spectrum of Third Window Abnormalities: Semicircular Canal Dehiscence and Beyond. AJNR Am J Neuroradiol 2017;38:2-9.

3. Rosowski JJ, Songer JE, Nakajima $\mathrm{HH}$, et al. Clinical, experimental, and theoretical investigations of the effect of superior semicircular canal dehiscence on hearing mechanisms. Otol Neurotol 2004;25:323-32.

4. Colebatch JG, Halmagyi GM, Skuse NF. Myogenic potentials generated by a click-evoked vestibulocollic reflex. J Neurol Neurosurg Psychiatry 1994;57:190-7.

5. Benamira LZ, Alzahrani M, Saliba I. Superior canal dehiscence: can we predict the diagnosis? Otol Neurotol 2014;35:338-43.

6. Brantberg K. Vestibular evoked myogenic potentials (VEMPs): usefulness in clinical neurotology. Semin Neurol 2009;29:541-7.

7. Brantberg K, Bergenius J, Tribukait A. Vestibular-evoked myogenic potentials in patients with dehiscence of the superior semicircular canal. Acta Otolaryngol 1999;119:633-40.

8. Hunter JB, Patel NS, O'Connell BP, et al. Cervical and Ocular VEMP Testing in Diagnosing Superior Semicircular Canal Dehiscence. Otolaryngol Head Neck Surg 2017;156:917-23.

9. Milojcic R, Guinan JJ Jr, Rauch SD, et al. Vestibular evoked myogenic potentials in patients with superior semicircular canal dehiscence. Otol Neurotol 2013; 34:360-7.

10. Roditi RE, Eppsteiner RW, Sauter TB, et al. Cervical vestibular evoked myogenic potentials (cVEMPs) in patients with superior canal dehiscence syndrome (SCDS). Otolaryngol Head Neck Surg 2009;141:24-8.

11. Streubel SO, Cremer PD, Carey JP, et al. Vestibular-evoked myogenic potentials in the diagnosis of superior canal dehiscence syndrome. Acta Otolaryngol Supp/ 2001;545:41-9.

12. Noij KS, Duarte MJ, Wong K, et al. Toward Optimizing Cervical Vestibular Evoked Myogenic Potentials (cVEMP): Combining Air-Bone Gap and cVEMP Thresholds to Improve Diagnosis of Superior Canal Dehiscence. Otol Neurotol 2018;39:212-220.

13. Prakash SR, Herrmann BS, Milojcic R, et al. Evaluating Inhibition of Motoneuron Firing From Electromyogram Data to Assess Vestibular Output Using Vestibular Evoked Myogenic Potentials. Ear Hear 2015;36:591-604.

14. Rauch SD, Zhou G, Kujawa SG, et al. Vestibular evoked myogenic potentials show altered tuning in patients with Meniere's disease. Otol Neurotol 2004; 25:333-8.

15. Taylor RL, Bradshaw AP, Halmagyi GM, et al. Tuning characteristics of ocular and cervical vestibular evoked myogenic potentials in intact and dehiscent ears. Audiol Neurootol 2012;17:207-18.

16. Manzari L, Burgess AM, McGarvie LA, et al. An indicator of probable semicircular canal dehiscence: ocular vestibular evoked myogenic potentials to high frequencies. Otolaryngol Head Neck Surg 2013;149:142-5.

17. van Tilburg MJ, Herrmann BS, Guinan JJ Jr, et al. Normalization reduces intersubject variability in cervical vestibular evoked myogenic potentials. Otol Neurotol 2014;35:222-7.

18. Noij KS, van Tilburg MJ, Herrmann BS, et al. Toward Optimizing VEMP: Calculating VEMP Inhibition Depth (VEMPid) with a Generic Template. Ear Hear 2018;39(6):1199-206.

19. Noij KS, Herrmann BS, Rauch SD, et al. Toward optimizing VEMP: Normalization Reduces the Need for Strong Neck Muscle Contraction. Audiol Neurotol 2017;22:282-291.

20. Rosengren SM, Govender S, Colebatch JG. Ocular and cervical vestibular evoked myogenic potentials produced by air- and bone-conducted stimuli: comparative properties and effects of age. Clin Neurophysiol 2011;122:2282-9.

21. Welgampola MS, Colebatch JG. Vestibulocollic reflexes: normal values and the effect of age. Clin Neurophysiol 2001;112:1971-9.

22. Brantberg K, Verrecchia L. Testing vestibular-evoked myogenic potentials with $90-\mathrm{dB}$ clicks is effective in the diagnosis of superior canal dehiscence syndrome. Audiol Neurootol 2009;14:54-8.

23. Janky KL, Nguyen KD, Welgampola M, et al. Air-conducted oVEMPs provide the best separation between intact and superior canal dehiscent labyrinths. Otol Neurotol 2013;34:127-34. 
24. Zhou G, Gopen Q, Poe DS. Clinical and diagnostic characterization of canal dehiscence syndrome: a great otologic mimicker. Otol Neurotol 2007;28:920-6.

25. Zuniga MG, Janky KL, Nguyen KD, et al. Ocular versus cervical VEMPs in the diagnosis of superior semicircular canal dehiscence syndrome. Otol Neurotol 2013;34:121-6.

26. Govender S, Fernando T, Dennis DL, et al. Properties of $500 \mathrm{~Hz}$ air- and bone-conducted vestibular evoked myogenic potentials (VEMPs) in superior canal dehiscence. Clin Neurophysiol 2016;127:252231.

27. Welgampola MS, Myrie OA, Minor LB, et al. Vestibular-evoked myogenic potential thresholds normalize on plugging superior canal dehiscence. Neurology 2008;70:464-72.

28. Fife TD, Colebatch JG, Kerber KA, et al. Practice guideline: Cervical and ocular vestibular evoked myogenic potential testing: Report of the Guideline Development, Dissemination, and Implementation Subcommittee of the American Academy of Neurology. Neurology 2017;89:2288-2296. 




\section{Chapter 4}

Clinical use of cVEMP in Menière's disease patients 



\section{Chapter 4.1}

Cervical vestibular evoked myogenic potentials in Menière's disease: A comparison of response metrics 


\section{Abstract}

\section{Objective}

The cervical vestibular evoked myogenic potential (cVEMP) has been used to evaluate patients with Menière's Disease (MD). Studied cVEMP metrics include: amplitude, threshold, frequency tuning and interaural asymmetry ratio (IAR). However, few studies compared these metrics in the same set of MD patients, and methodological differences prevent such a comparison across studies. This study investigates the value of different CVEMP metrics in distinguishing one set of MD patients from age-matched controls.

\section{Study design}

Prospective study.

\section{Setting}

Tertiary care center.

\section{Patients}

Thirty patients with definite unilateral MD and 23 age-matched controls were prospectively included. All underwent cVEMP testing at 500, 750, 1000 and $2000 \mathrm{~Hz}$ on each side. Ears were separated into three groups: affected MD, unaffected MD, and control.

\section{Main outcome measures}

Sound level functions were obtained at each frequency, and normalized peak-to-peak amplitude (VEMPn), VEMP inhibition depth (VEMPid), threshold, frequency-tuning ratio and IAR were calculated. For all metrics, the differentiation between MD and control ears was compared using receiver operating characteristic (ROC) curves.

\section{Results}

$500 \mathrm{~Hz}$ cVEMP threshold, VEMPn, and VEMPid were similarly good at distinguishing affected MD ears from healthy ears, with ROC area under the curves (AUCs) of more than 0.828 and optimal sensitivities and specificities of at least $80 \%$ and $70 \%$. Combinations of these three metrics yielded slightly larger AUCs $(>0.880)$. Tuning ratios and IAR were less effective in separating healthy from affected ears with AUCs ranging from 0.529 to 0.720 .

\section{Conclusion}

The cVEMP metrics most useful in distinguishing MD patients from healthy controls are threshold, VEMPn, and VEMPid, using $500 \mathrm{~Hz}$ stimuli. 


\section{Introduction}

The cervical vestibular evoked myogenic potential (cVEMP) is used to evaluate the function of the saccule and inferior vestibular nerve. During this test, the saccule is acoustically or mechanically stimulated resulting in a primarily ipsilateral inhibition of the sternocleidomastoid (SCM) muscle that is measured by SCM electromyography (EMG) (1,2).

cVEMPs have been studied in patients with superior semicircular canal dehiscence syndrome (SCD) and Menière's disease (MD) (3-6). In SCD patients, the CVEMP seems a promising screening tool achieving high sensitivities and specificities (7). The value of cVEMP in differentiating MD patients from healthy subjects is less clear. Most studies agree that MD patients have lower cVEMP amplitudes $(8-14)$, higher thresholds $(3,14)$, altered frequency tuning $(3,8,11,13-17)$, and higher interaural asymmetry ratios (IARs) $(8,14,18)$. There is, however, considerable overlap in cVEMP metrics between MD patients and healthy subjects. The variety of methods used to obtain, analyze and evaluate cVEMPs makes it challenging to compare studies. For example, most studies investigating CVEMP in MD patients did not describe methods to normalize cVEMPs to account for differences in muscle contraction $(3,10,13,15,16,18-21)$. Muscle contraction has a large effect on cVEMP amplitude (1,22-27). Finding either a difference or no difference between groups using non-normalized amplitudes could potentially be due to differences in muscle contraction between groups. Studies that failed to normalize cVEMPs for muscle contraction rarely compare muscle contractions between groups, which makes these studies difficult to interpret and compare. Furthermore, many studies evaluating MD patients lack an age-matched control group $(3,9,13,16,17,19)$. cVEMP amplitudes decrease, and thresholds increase with age (22,28-30). Because similar changes are seen in MD patients, it is essential to include an age-matched control group.

Several studies of cVEMP metrics in MD patients describe sensitivities and specificities and/or provide receiver operating characteristic (ROC) curves and areas under the curves (AUCs) $(8,11,15,18,19,21)$. The evaluated metrics differed across studies and included $500 / 1000 \mathrm{~Hz}$ or $1000 / 500 \mathrm{~Hz}$ amplitude ratios $(8,11,18)$, shifting of the most sensitive frequency (lowest threshold) to $1000 \mathrm{~Hz}$ (15), a combination of amplitudes, $500 / 1000 \mathrm{~Hz}$ ratio, IARs and audiogram data (18), and assessing cVEMPs as "normal" versus "abnormal" $(19,21)$.

The use of many different metrics, inconsistent use of normalization across studies, and the lack of healthy age-matched controls in many studies make it difficult to compare the results from these studies to determine the value of cVEMPs in detecting saccular dysfunction in MD patients. The present study compares the usefulness of different cVEMP metrics in differentiating one set of MD patients from healthy age-matched controls by providing sensitivities and specificities for each metric. For reliable 
comparison, all metrics were obtained in the same patients and controls, using the same methods.

\section{Methods}

\section{Subjects}

Thirty patients with definite unilateral Menière's disease, per Lopez-Escamez et al. 2015, and 23 age-matched controls were prospectively included (31). The Lopez et al. criteria for definite Menière's Disease include: 1 ) the presence of two or more spontaneous episodes of vertigo, each lasting 20 minutes to 12 hours, 2) audiometrically documented low- to medium frequency sensorineural hearing loss in one ear, defined as a bone-conducted threshold of at least $30 \mathrm{~dB} H \mathrm{HL}$ at each of two contiguous frequencies below $2000 \mathrm{~Hz}$, on at least one occasion, 3) fluctuating hearing loss, tinnitus and/or aural fullness in the affected ear and 4) not better accounted for by another diagnosis (31). Patients who previously received invasive treatment, including intratympanic gentamicin or corticosteroid injections and surgery were excluded. Exclusion criteria for the healthy control group were a self-reported history of hearing loss, vertigo and/or balance problems. This study was approved by the Human Studies Committee of the Massachusetts Eye and Ear Infirmary (\#13-097H, PI: S.D. Rauch).

\section{Audiometry}

All subjects underwent pure tone audiometry with air- and bone-conduction tonal thresholds measured at octave frequencies from 250 to $4000 \mathrm{~Hz}$. If the difference between air- and unmasked bone-conduction thresholds was larger than $10 \mathrm{~dB} \mathrm{HL}$, bone-conduction thresholds were masked. The air-bone gap (ABG) was calculated at each tested frequency by subtracting the bone-conduction threshold from the airconduction threshold. Patients and healthy subjects with an $A B G>10 \mathrm{~dB}$ at any of the frequencies used for cVEMP testing $(500,750,1000$ and $2000 \mathrm{~Hz})$ were excluded from this study, because this could indicate the presence of middle ear pathology, which can influence cVEMP outcomes (decrease amplitude and increase threshold).

\section{cVEMP}

A custom-programmed evoked potential system was used to generate tone bursts and record CVEMPs. The time between the start of symptoms and the obtained CVEMP varied from 12 to 333 months (average: 83 months). During CVEMP testing, subjects sat up straight and turned their head away from the stimulated ear to contract the ipsilateral SCM (i.e., the left ear alone was acoustically stimulated while contracting the left SCM). EMG activity was recorded from four surface electrodes: a non-inverting 
electrode on the middle belly of each SCM, an inverting electrode at the midpoint between SCM attachments to the sternum, and a ground electrode on the midline forehead. Ipsilateral SCM EMG was monitored while subjects contracted their SCM to produce more than $45 \mu \mathrm{V}$ root mean square (rms) EMG. This minimum muscle contraction of $45 \mu \mathrm{V}$ rms was chosen based on a previous study, which concluded that for contractions that produced $45-300 \mu \mathrm{V}$ rms EMG, the contraction strength had little effect on the metrics used in the current study (normalized peak-to-peak amplitude, VEMP inhibition depth and CVEMP detection) (22). EMG activity was amplified and bandpass filtered between 10 and $750 \mathrm{~Hz}$ using the bioamplifier of the Eclipse EP15 (Interacoustics). The output of the bioamplifier was sampled at $50 \mathrm{kHz}$ with a 16-bit analog-to-digital converter (National Instruments).

cVEMPs were elicited using 500, 750, 1000 and $2000 \mathrm{~Hz}$ tone bursts generated by custom-programmed evoked-potential software (National Instruments 16-bit digital I/O board) using a Blackman gating function with two cycle rise and fall times (4.0 ms at $500 \mathrm{~Hz}, 2.5 \mathrm{~ms}$ at $750 \mathrm{~Hz}, 2 \mathrm{~ms}$ at $1000 \mathrm{~Hz}, 1 \mathrm{~ms}$ at $2000 \mathrm{~Hz}$ ) and no plateau. Tone bursts were presented monaurally via circumaural headphones (Telephonics TDH-49) at a repetition rate of 13 bursts/s. At least 200 cVEMP responses were obtained and averaged for each recording. In the MD group, tone bursts were presented at 103, 113, 123 and $128 \mathrm{~dB}$ peak sound pressure level (peSPL), while in the healthy control group, tone bursts were presented at 93,103, 113 and $123 \mathrm{~dB}$ peSPL (123 dB peSPL is equivalent to $90 \mathrm{~dB} \mathrm{nHL}$ ). This protocol difference between the MD and healthy control group was chosen because MD patients generally have higher cVEMP thresholds. Numerical values for sound level, frequency, and side were placed in a table which was randomized (separately for each subject) across all three variables and which set the presentation order for these variables.

\section{cVEMP metrics}

The collection of sound level functions at all frequencies and the saving of all individual responses allowed for the calculation of multiple metrics, including VEMPn (normalized peak-to-peak amplitude), VEMPid (VEMP inhibition depth), threshold, frequency tuning and interaural asymmetry ratio (IAR).

\section{VEMPn}

We have recently completed a study comparing different methods of normalizing cVEMPs and found that several methods were equivalently good while others were inferior (32). Here we make use of these results and use just one of the good methods, which serves a proxy for all of the good normalization methods. Van Tilburg et al. (32), found that the best CVEMP normalizations used EMG quantification from individualtrace EMGs, either by averaging the rectified or rms EMG and applying this average EMG to normalize the cVEMP waveform, or by applying the EMG trace by trace. 
Rectified and rms EMG were usually equivalent. In addition, EMG measurement windows close to the time of the CVEMP response were best, and inclusion of the time when cVEMP occurred had a negligible effect because the cVEMP modulation is a small fraction of the EMG amplitude and the EMG amplitude near the CVEMP time is the most relevant. In contrast, normalizing the average cVEMP amplitude by an EMG metric derived from the averaged cVEMP waveform (e.g. from the 20 or $50 \mathrm{~ms}$ period before the VEMP response) was distinctly inferior. Since the good normalization methods were equivalent, we use only one of them.

Trace-by-trace normalization was performed, meaning that each raw EMG trace was divided by the overall, $77 \mathrm{~ms}$ long rms value of the same trace (33). VEMPn was the amplitude difference between the first positive (P1) and the first negative (N1) peak of the average normalized cVEMP waveform (33).

\section{VEMPid}

VEMPid is a metric that estimates the percentage reduction in spike-rate of SCM motoneurons elicited by acoustic stimulation of the saccule. This metric uses a template correlation method (34). The VEMPid is larger when the CVEMP response is strong and smaller when the CVEMP response is weaker. In this study, VEMPid was calculated using a generic template created from cVEMP responses of healthy subjects (35). The first step in computing VEMPid, is to calculate template correlation values (TCVs) using the point-by-point correlation of each individual cVEMP trace with the generic template. VEMPid was then calculated by dividing the mean of all (at least 200) TCVs by the standard deviation of the TCVs and multiplying by 0.2 . While the original VEMPid calculation used a subject-specific template, it was later determined that VEMPid can be calculated using a generic template if the generic template's latency is set to the latency of the patient's response $(34,35)$. In patients, the use of a generic template is preferred over a subject-specific template because patients may not have a robust cVEMP suitable for a template.

\section{Threshold}

After cVEMPs at the four preset sound levels were obtained, additional $5 \mathrm{~dB}$ steps were obtained and if necessary repeated, to find the threshold (the lowest sound level at which a cVEMP was present). If no CVEMP was present at our equipment limit (133 dB peSPL), threshold was defined as $10 \mathrm{~dB}$ higher than this limit.

\section{Frequency tuning}

cVEMP tuning was evaluated using VEMPn and threshold. 500/1000 Hz ratios were calculated using these metrics. VEMPid was not used to calculate $500 / 1000 \mathrm{~Hz}$, because 
this metric is not suitable for such a computation (VEMPid can be negative and/or very small).

IAR

The IAR was calculated using VEMPn. In previous studies a variety of formulae have been used to compute the IAR $(8,12,16,18-21,36,37)$ and we decided to use all options in the current study:

Healthy control group:

$$
\begin{aligned}
& \text { Control L-R: } \quad \text { IAR }=100 * \frac{\text { VEMPn left }- \text { VEMPn right }}{\text { VEMPn left }+ \text { VEMPn right }} \\
& \text { Control R-L: } \quad \text { IAR }=100 * \frac{\text { VEMPn right }- \text { VEMPn left }}{\text { VEMPn right }+ \text { VEMPn left }} \\
& \text { Control Abs: } \quad I A R=100 * \frac{\mid \text { VEMPn left }- \text { VEMPn right }}{\text { VEMPn left }+ \text { VEMPn right }}
\end{aligned}
$$

MD group:

$$
\begin{array}{ll}
\text { MD U-A: } & \text { IAR }=100 * \frac{\text { VEMPn unaffected }- \text { VEMPn affected }}{\text { VEMPn unaffected }+ \text { VEMPn affected }} \\
\text { MD Abs: } & \text { IAR }=100 * \frac{\mid \text { VEMPn unaffected }- \text { VEMPn affected }}{\text { VEMPn unaffected }+ \text { VEMPn affected }}
\end{array}
$$

Controls Abs and MD Abs represent the absolute values used in the corresponding formulae.

\section{Data analysis}

MD and control-group ages were compared using an independent-samples $t$ test. Audiometric data and cVEMP metrics (VEMPn, VEMPid, threshold, 500/1000 Hz ratios, and IAR) among affected, unaffected and healthy control ears were compared using full-factorial analyses of variance (ANOVA) with group and frequency considered fixed factors and subject considered a random factor. Post hoc pairwise group comparisons used Bonferroni adjustment for multiple comparisons. The ability of each cVEMP metric to distinguish between affected and healthy control ears was assessed with ROC curves. Statistical analyses were performed using SPSS (version 24.0; Chicago, IL). A $p$ value of $<0.05$ was considered statistically significant. When a Bonferroni correction was used, the significance criterion was 0.05 divided by the number of comparisons. 


\section{Results}

\section{Patient characteristics}

Thirty patients with unilateral definite Menière's disease (31) were included (12 women, mean age: 55.6 years; range: $28-75$ years). Ears were separated into two groups: affected and unaffected. Additionally, 23 age-matched healthy controls were included (12 women, mean age: 54.8 years; range: 33-73 years). No significant difference in age was found between control and MD groups $(p=0.783)$.

\section{Audiograms}

Bone-conduction thresholds were recorded for all groups (Figure 4.1.1A). Average lowfrequency bone-conduction thresholds were calculated using 250, 500 and $1000 \mathrm{~Hz}$ data (Figure 4.1.1B). Affected ears had significantly higher average low-frequency boneconduction thresholds compared to the unaffected and healthy control ears, as did unaffected vs. control ears ( $p<0.001$ for each comparison).

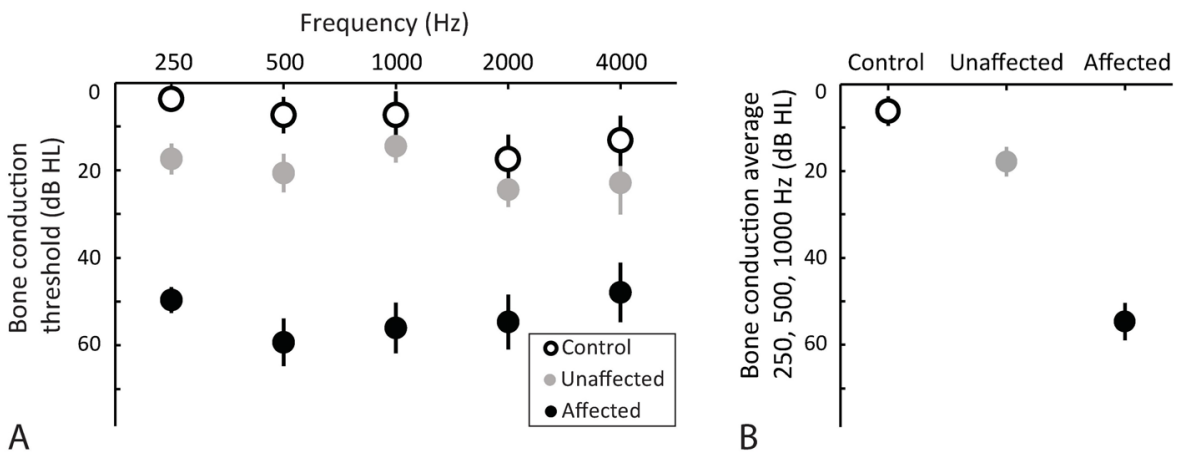

Figure 4.1.1 Average bone-conduction threshold for each group and frequency (A). Low-frequency boneconduction thresholds (averaged across 250, 500 and $1000 \mathrm{~Hz}$ ) for each group (B). Error bars indicate $95 \%$ confidence intervals.

\section{cVEMP}

On average, VEMPn and VEMPid were largest in control ears, next largest in unaffected ears and smallest in affected ears (Figure 4.1.2). To evaluate differences in VEMPn and VEMPid among the three groups, we used the highest sound level available for all groups (123 dB peSPL).

For VEMPn, there was a significant interaction between group and frequency ( $\mathrm{F}=$ 2.979, $p=0.007$; Figure 4.1.2). At 500, 750 and $1000 \mathrm{~Hz}$, VEMPn was significantly higher in control vs. affected ears $(p<0.001$ for each frequency; Bonferroni-adjusted 
significance criterion is 0.0167$)$. For $500 \mathrm{~Hz}$, VEMPn was significantly higher in control vs. unaffected ears $(p<0.001)$, and the difference observed between affected and unaffected ears (Figure 4.1.2) did not reach significance $(p=0.064)$. At 750 and 1000 $\mathrm{Hz}$, differences that did not reach Bonferroni-adjusted significance were also observed for control vs. unaffected ears ( $p=0.044$ and $p=0.052$ ) and for affected vs. unaffected ears $(p=0.031$ and $p=0.127)$. At $2000 \mathrm{~Hz}$, no significant differences in VEMPn were found for any group combinations.

For VEMPid, there was also a significant interaction between group and frequency $(\mathrm{F}=$ 2.170, $p=0.045$; Figure 4.1.2). At 500, 750 and $1000 \mathrm{~Hz}$, VEMPid was significantly larger in control vs. affected ears ( $p<0.001$ for each frequency; Bonferroni-adjusted significance criterion is 0.0167). For 500 and $1000 \mathrm{~Hz}$, VEMPid was significantly higher in control vs. unaffected ears ( $p<0.001$ and $p=0.011$ ), while the difference between affected and unaffected ears at these frequencies did not reach significance ( $p=0.078$ and $p=0.129$ ). For $750 \mathrm{~Hz}$, the difference between control and unaffected ears ( $p=$ $0.041)$ and between affected and unaffected ears $(p=0.038)$ did not meet the Bonferroni-adjusted significance criterion. At $2000 \mathrm{~Hz}$, no significant differences in VEMPid were found for any group combinations.

On average, cVEMP thresholds were highest in the affected ears, followed by unaffected ears and were lowest in control ears (Figure 4.1.3). A significant interaction was found between group and frequency $(F=0.268, p=0.014)$. For 500,750 and 1000 $\mathrm{Hz}$, threshold was significantly lower in control vs. affected ears $(p<0.001$ for each frequency) and for control vs. unaffected ears ( $p<0.001, p=0.003$ and $p=0.002$ respectively). No significant difference in threshold was found between affected and unaffected ears at any frequency. At $2000 \mathrm{~Hz}$ no significant threshold differences were found for any group combinations. 

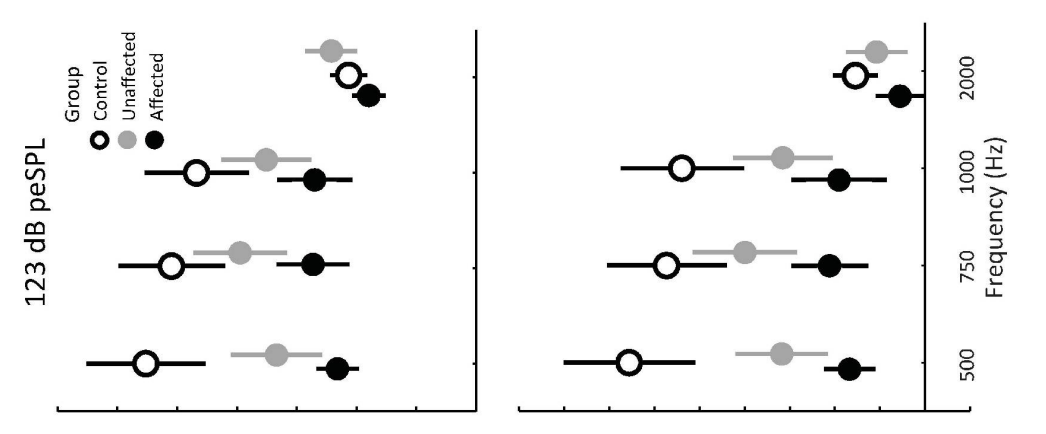

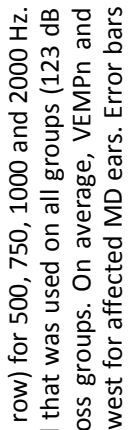

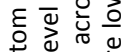
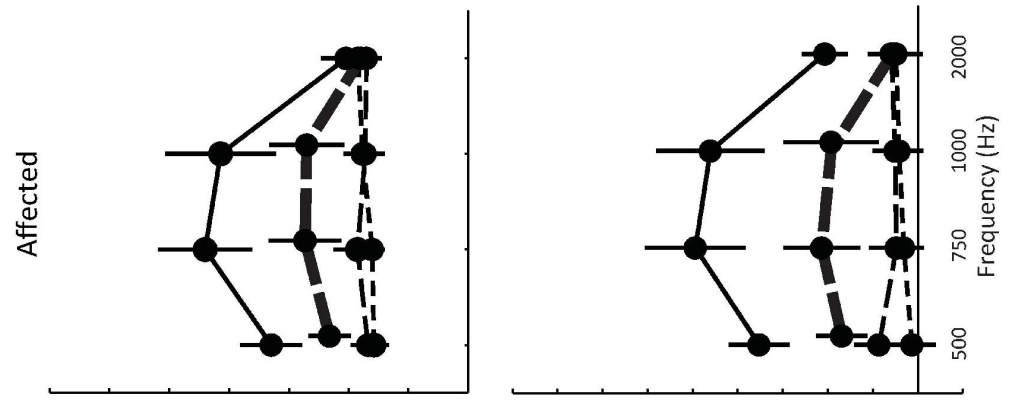

응 윙

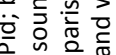

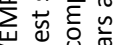

$\geq \frac{1}{2}$

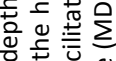

응 30.

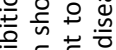

此

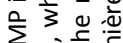

包吉离
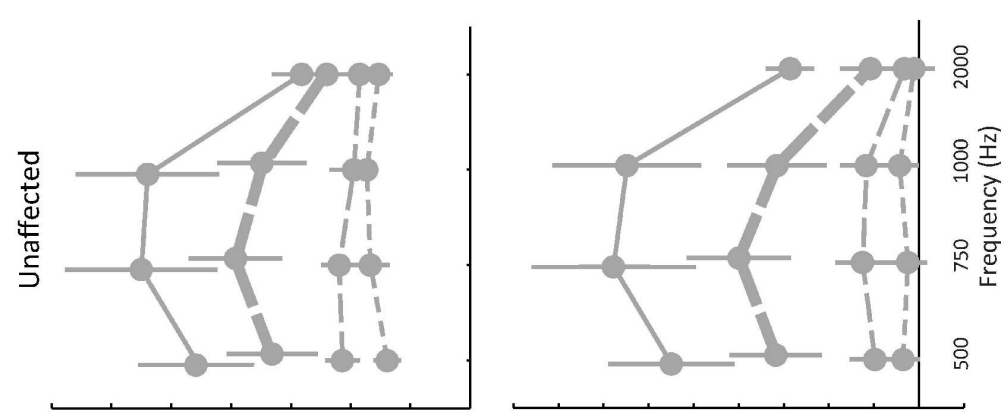

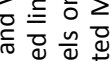

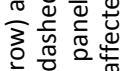

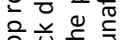

는

을 뭉 뭉

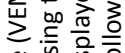

은 는

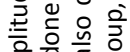

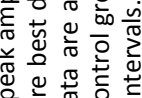
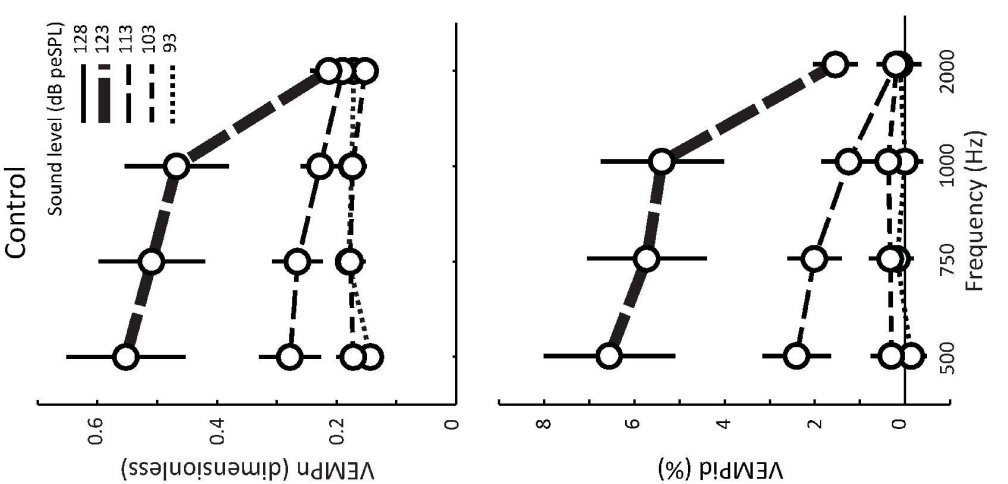

논

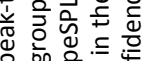

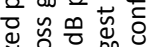

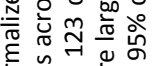

돋ำ

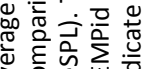

过

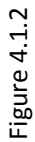




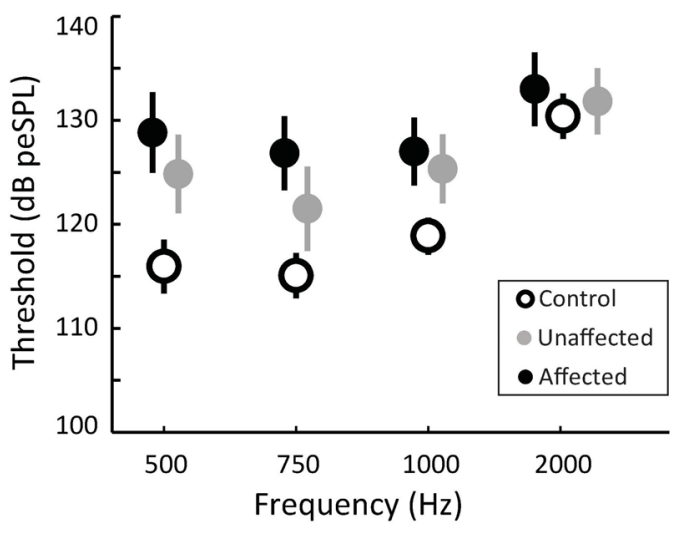

Figure 4.1.3 Average cVEMP threshold for the control (open circles), unaffected (grey circles), and affected (black circles) groups at 500, 750, 1000 and $2000 \mathrm{~Hz}$. Error bars indicate the 95\% confidence intervals. cVEMP indicates cervical vestibular evoked myogenic potential.

\section{Tuning}

Half of the affected ears had a higher threshold at $500 \mathrm{~Hz}$ than at $1000 \mathrm{~Hz}$ (Figure 4.1.4A). In the unaffected ears, thresholds at 500 and $1000 \mathrm{~Hz}$ were most often equal (Figure 4.1.4A). In contrast, most control subjects had a higher threshold at 1000 $\mathrm{Hz}$ than at $500 \mathrm{~Hz}$ (Figure 4.1.4A). For the $500 / 1000 \mathrm{~Hz}$ threshold ratio (Figure 4.1.4B), a significant effect of group was found $(F=4.300, p=0.016)$, and pairwise comparisons revealed significantly higher threshold ratios in affected vs. control ears $(p=0.005$; Bonferroni adjusted criterion is 0.0167$)$. The differences in threshold ratio between affected and unaffected ( $p=0.173$ ) or unaffected and control ears $(p=0.157)$ were not significant. For VEMPn tuning, $123 \mathrm{~dB}$ peSPL recordings were used to calculate the $500 / 1000 \mathrm{~Hz}$ ratio and no significant differences were found between groups ( $F=1.348$, $p=0.264$ ) (Figure 4.1.4C). 

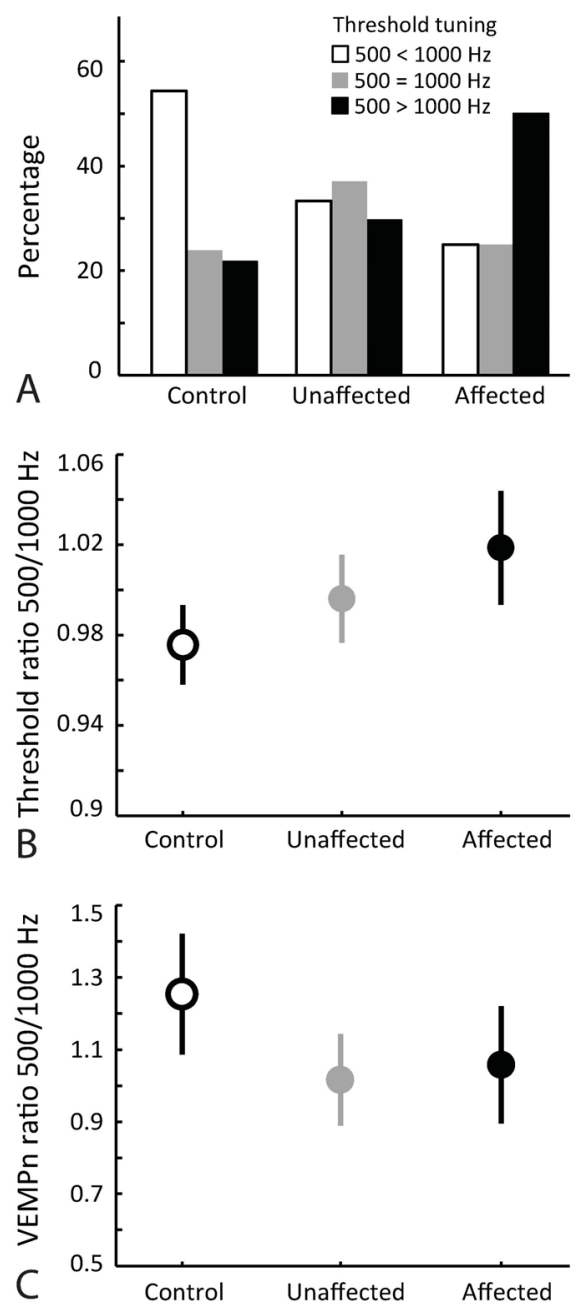

Figure 4.1.4 cVEMP frequency tuning varied across groups. Group threshold tuning was compared by the fraction of ears with $500 / 1000 \mathrm{~Hz}$ ratios below, at, and above unity (A), and by average $500 / 1000 \mathrm{~Hz}$ threshold (B) and VEMPn (C) ratios. Error bars indicate the $95 \%$ confidence intervals. cVEMP indicates cervical vestibular evoked myogenic potential.

IAR

VEMPn IAR was studied using the $123 \mathrm{~dB}$ peSPL recordings. ANOVA found no significant effect of frequency $(F=0.213, p=0.887)$, therefore, data were pooled across frequencies. To include all of the calculations used in previous reports, five IAR formulae were used (see Methods). Formulae using ear-differences always yielded 
smaller IARs than formulae using the absolute value of the difference (Figure 4.1.5). For both types of formulae, the MD group had higher IARs than the control group at 500, 750 and $1000 \mathrm{~Hz}$. In controls, the right ears had slightly higher VEMPn, and IAR reversed sign for formulae Control L-R vs. Control R-L. Comparing control and MD groups, Control L-R vs. MD U-A groups were significantly different $(p<0.001$, Bonferroniadjusted criterion is 0.0167$)$, but Control R-L vs. MD U-A groups ( $p=0.077)$, Control Abs vs. MD U-A groups $(p=0.018)$ and Control Abs vs. MD Abs were not $(p=0.321)$.

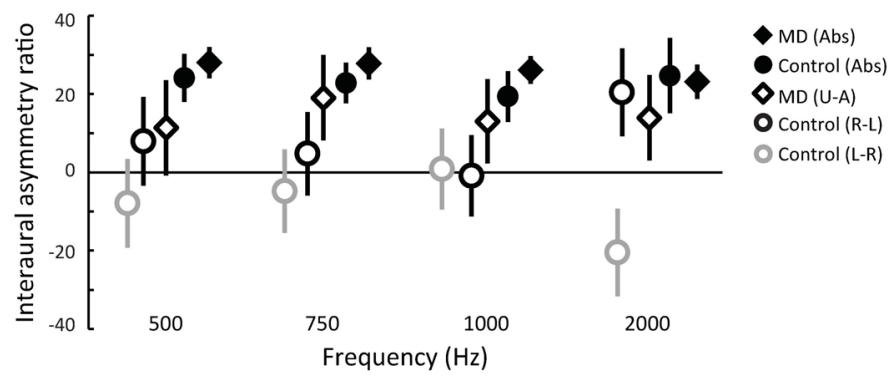

Figure 4.1.5 Interaural asymmetry ratios (IAR) calculated in five ways (see Methods). Symbols indicate average IARs using the $123 \mathrm{~dB}$ peSPL data for all frequencies; error bars indicate $95 \%$ confidence intervals. See Methods for formulae: Abs indicates the absolute values of the differences were used.

\section{ROC curves}

To evaluate the ability of the cVEMP metrics to distinguish affected MD ears from control ears we created ROC curves. The $2000 \mathrm{~Hz}$ data were not included because no significant differences between groups were found using this frequency (Figures 4.1.2 and 4.1.3). Based on the ROC area under the curve (AUC), $500 \mathrm{~Hz}$ was the best frequency to distinguish groups for threshold ( $A U C=0.828)$, VEMPn (AUC $=0.846$ ) and VEMPid (AUC $=0.854$ ) (Figure 4.1.6). The sensitivities and specificities that could be achieved with these metrics were also similar, with sensitivities of $80.0,82.6$ and $80.4 \%$ respectively, and corresponding specificities of 76.1, 70.0 and $73.3 \%$ (Figure 4.1.6). Both threshold tuning ratio and VEMPn tuning ratio were less valuable metrics, with AUCs of 0.697 and 0.578 (Figure 4.1.6). The IAR was also inferior as evaluated by ROC curves for 1) Control L-R vs. MD U-A, 2) Control R-L vs. MD U-A, 3) Control Abs vs. MD U-A, and 4) Control Abs vs. MD Abs. The 500, 750 and $1000 \mathrm{~Hz}$ AUCs for each combination were 1) $0.659,0.720,0.646,2) 0.533,0.622,0.641,3) 0.639,0.567,0.557$ and 4) $0.529,0.525$ and 0.600 (ROC curves not shown).

The tuning ratio and IAR analyses used only $123 \mathrm{~dB}$ peSPL recordings. However, the MD group also had $128 \mathrm{~dB}$ peSPL recordings available. We calculated ROC curves from tuning ratios and IARs comparing MD $128 \mathrm{~dB}$ peSPL recordings vs. control $123 \mathrm{~dB}$ peSPL 
recordings. These analyses yielded results similar to those from all-123-peSPL comparisons (data not shown).
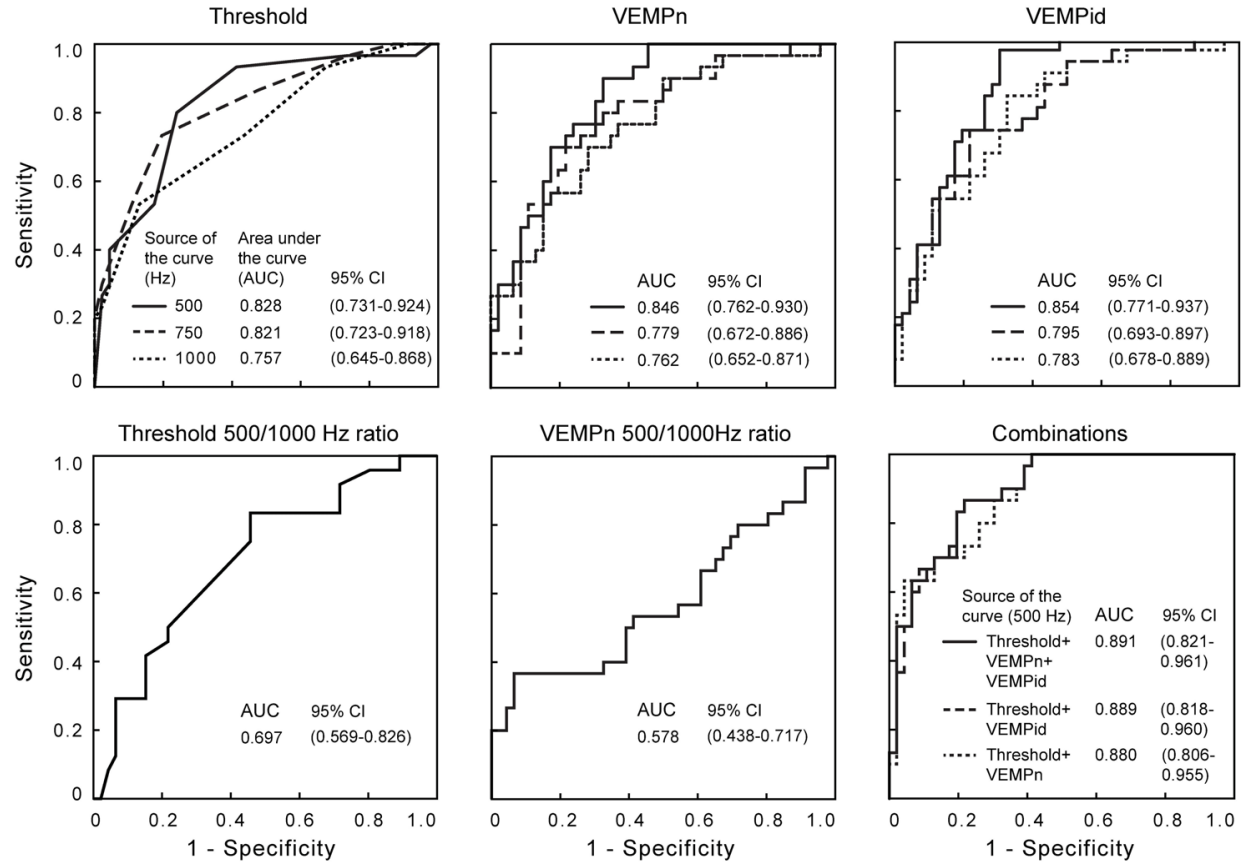

Figure 4.1.6 Receiver operating characteristic (ROC) curves displaying the ability of various cVEMP statistics to separate Menière's from normal ears. Each panel shows the sensitivity of detecting Menière's disease (the true-positive rate) versus the false-positive rate ( 1 - specificity) for 500 , 750 and $1000 \mathrm{~Hz}$ data at $123 \mathrm{~dB}$ peSPL. Top-row ROC statistics are threshold, normalized peakto-peak amplitude (VEMPn) and VEMP inhibition depth (VEMPid). Bottom-row ROC statistics are $500 / 1000 \mathrm{~Hz}$ threshold ratio, VEMPn $500 / 1000 \mathrm{~Hz}$ ratio and ROCs created by combinations of the top-row metrics. Because the $500 \mathrm{~Hz}$ top-row data yielded the largest AUCs, this frequency was used to evaluate combinations of these metrics (bottom right panel). In each panel, the area under the curve (AUC) and 95\% confidence interval (CI) of each line are displayed.

Because the $500 \mathrm{~Hz}$ data for threshold, VEMPn and VEMPid gave the best results, we explored if a combination of these three metrics would improve the ability to distinguish MD-affected from control ears. The metrics were combined using logistic regression models. The following combinations were used: 1) Threshold and VEMPn, 2) Threshold and VEMPid, 3) Threshold, VEMPn and VEMPid, providing the following logistic regression models:

1) $\quad \beta 1=-7.428+($ Threshold*0.078) $+($ VEMPn*-7.291)

2) $\quad \beta 2=-7.480+($ Threshold*0.072) $+($ VEMPid*-0.514)

3) $\quad \beta 3=-7.258+($ Threshold*0.072) $+($ VEMPn*-1.888) $+($ VEMPid*-0.396) 
These equations were used to calculate the beta $(\beta)$ for each subject. The $\beta$ values were then used to create ROC curves for each combination of metrics, yielding the following AUCs: 1) $0.880,2) 0.889$, and 3) 0.891 (Figure 4.1.6). Corresponding sensitivities and specificities were: 1) $80 \%$ and $73.9 \%$, 2) $83.3 \%$ and $80.4 \%$ 3) $83.3 \%$ and $80.4 \%$.

\section{Discussion}

The purpose of this study was to investigate how well different cVEMP metrics separate MD from normal ears. To reliably compare these metrics, all were obtained in the same MD patients and age-matched controls, with consistent methods used to obtain and analyze the CVEMP.

For distinguishing affected-MD from control ears, cVEMP threshold, VEMPn and VEMPid metrics at $500 \mathrm{~Hz}$ did a similarly good job based on their ROC curves, with AUCs of $>0.828$ and sensitivities and specificities of at least $80 \%$ and $70 \%$. Combinations of the best three metrics ( $500 \mathrm{~Hz}$ : cVEMP threshold, VEMPn and VEMPid) yielded slightly larger AUCs (>0.880) sensitivities and specificities (Figure 4.1.6). Results equivalent to our VEMPn results are expected to be produced by any CVEMP normalization done by rms or rectified EMG from individual traces, whereas normalizations by an EMG metric derived from the averaged CVEMP waveform are expected to be less effective (32). Tuning ratios and IAR were less effective in separating healthy from affected ears with AUCs 0.529-0.720 (Figure 4.1.6).

Although VEMPid has produced results that may seem similar to VEMPn, these are completely different metrics. VEMPn measures the size of a cVEMP while correcting for the effect of muscle contraction and VEMPid estimates the inhibition depth of the saccule based on a computational model applied to the cVEMP recordings (34). Although the metrics may produce similar results, the VEMPid is easier to interpret, because it goes down to zero when no response is present $(22,35)$. Although yet to be proven, VEMPid has the potential of reducing inter-tester variability in determining cVEMP threshold. VEMPid measurements may also aid in determining when to stop recording, which would make testing time shorter and decrease patient discomfort (35).

In the current study, well-defined MD patients were compared with healthy controls to investigate how well cVEMP detects saccular dysfunction. Clinically, the ability to detect saccular dysfunction with cVEMPs could potentially be used to identify patients whose symptoms are suggestive, but not diagnostic for $\mathrm{MD}$, as well as to track saccular function over time (38). It appears that different stages of MD may be accompanied by different CVEMP outcomes, with an increase in CVEMP thresholds for patients with more advanced disease $(39,40)$. Therefore, early stage MD patients may require separate consideration from those included here. Our comparison of well-defined MD patients with healthy controls is a step in this direction and suggests that CVEMP 
recordings may yield valuable information. A good next step would be to follow unilateral MD patients over time to determine the value of CVEMP metrics obtained from the non-symptomatic ear in predicting which patients will develop MD in this ear. To investigate how well the cVEMP metrics used in this study are in detecting Menière's ears from those with other clinical problems, more work is needed in which a similar analysis is performed in patient groups with non-MD clinical presentations. For all of these potential uses of cVEMP measurements, the present study provides a first step in identifying which cVEMP metrics to focus on in future studies.

\section{Comparison with previous studies}

Previous studies evaluating AUCs and/or sensitivities and specificities to separate MD from healthy ears used a variety of metrics $(8,11,15,18,19,21)$. It is difficult to compare results of these studies with our results because different patient groups and methods were used to obtain and analyze the cVEMP. Nonetheless, an overview of the analyzed metrics and results can be presented:

1) $500 / 1000 \mathrm{~Hz}$ or $1000 / 500 \mathrm{~Hz}$ amplitude ratio (Maxwell et al. 2017 (18): AUC <0.7; Salviz et al. 2015 (11) and 2016 (8): AUC=0.731, sensitivity $=76 \%$ and specificity $=$ $80 \%$ )

2) Shifting of most sensitive frequency (i.e. lowest threshold) to $1000 \mathrm{~Hz}$ (Zhu et al. 2014 (15): sensitivity $=47 \%$, specificity $=64 \%$ )

3) A combination of amplitude $500 / 1000 \mathrm{~Hz}$ ratio and asymmetry ratio (Maxwell et al. 2017 (18): AUC = 0.814) and audiogram data (Maxwell et al. 2017 (18): AUC = 0.906 , sensitivity $=64 \%$, specificity $=93 \%$ )

4) Qualifying cVEMP as normal versus abnormal, "abnormal": Decreased or absent response (Egami et al. 2013 (19): sensitivity = 50\%, specificity $=48.9 \%$ ); absent response and/or interaural asymmetry $>34 \%$ (Lamounier et al. 2017 (21): sensitivity $=63.5-63.6 \%$, specificity $=84.6-93.7 \%$ )

The Maxwell et al. (18) study results are the most similar to ours. The AUCs found by Maxwell et al., using a combination of CVEMP and oVEMP $500 / 1000 \mathrm{~Hz}$ amplitude ratio as well as $500 \mathrm{~Hz}$ and $1000 \mathrm{~Hz}$ CVEMP asymmetry ratio, are similar to the AUCs we found for $500 \mathrm{~Hz}$ threshold, VEMPn and VEMPid as separate metrics (and which required cVEMP testing only). Maxwell et al. performed an additional analysis adding audiometric data to the previously mentioned combination, yielding an AUC of 0.906 . We did not include audiometric data in our ROC analyses, because patients were preselected based on their audiometric data (see Methods, Lopez-Escamez et al. (31) criteria for definite MD). Of note, the Maxwell et al. (18) study included patients with definite, probable and possible MD and did not mention the use of a normalization technique to correct for differences in muscle contraction.

Comparison of our results to Egami et al. (19) and Lamounier et al. (21) is difficult. Their rules of what is normal or abnormal are arbitrary, which makes their outcomes 
challenging to interpret. Our ROC curves created with raw data of each metric and directly comparing MD with normal groups provides clearly interpretable results. Other institutions can repeat our analysis and compare their results to ours.

Consistent with previous reports, we found a shift in frequency tuning in MD ears. Most affected ears had higher thresholds at $500 \mathrm{~Hz}$ than at $1000 \mathrm{~Hz}$, while the majority of controls had higher thresholds at $1000 \mathrm{~Hz}$ than at $500 \mathrm{~Hz}(3,15)$. However, for 500/1000 $\mathrm{Hz}$ ratios, a significant difference was only found for threshold ratios between affected and control ears. Control ears had higher VEMPn 500/1000 Hz ratios compared to MDaffected and MD-unaffected ears, but this did not reach the significance level. In contrast to our findings, previous reports did find significant differences between affected, unaffected and/or control ears regarding tuning $(8,12,18)$. Differences between our study and that by Maxwell et al. have been previously described and the methodological differences could explain the difference in findings. Similarly, Taylor et al. did not mention the use of a normalization technique to control for differences in muscle contraction, which might explain the disconcordance with our results (14). In contrast, Salviz et al. (8) only included patients with definite MD, used an age-matched control group and normalized for muscle contraction. It is unclear why our results differ from theirs.

A report from Zhu et al. (15) included patients with non-MD vestibular pathologies (including migraine related dizziness, BPPD, chronic subjective dizziness syndrome, vestibular neuritis and labyrinthitis) to study the effect of cVEMP threshold tuning. This study found that a "tuning curve shift" defined as the most sensitive frequency being $1000 \mathrm{~Hz}$ provided an AUC of 0.560 . This study did not look at 500/1000 Hz amplitude ratios which makes it difficult to compare to the other studies. The low AUC value found does not make the use of frequency tuning more promising, and indicates that frequency tuning changes similar to those in MD patients can occur in other vestibular pathologies.

The IAR is a widely used metric to evaluate MD patients, so we included it in this study even though we do not recommend its use in MD patients. Both the current study and previous studies have found that CVEMPs from the asymptomatic ears of unilateral MD patients are different from cVEMPs from normal ears, and the difference is in the direction of the asymptomatic ears being more like symptomatic MD ears, suggesting possible occult changes in the asymptomatic ear of some unilateral MD patients (3). The abnormal cVEMPs in asymptomatic ears of unilateral MD patients would reduce the IAR and make MD-IARs more like IARs from normal ears and thus less useful in detecting MD ears from normal ears. This hypothesis was confirmed by the low AUCS calculated from IARs which ranged from 0.525 to 0.720 . The exact AUC value depends on which IAR formula was used, but all of these values are inferior to those obtained by other CVEMP metrics. For this reason, and because approximately one quarter of MD patients suffer from bilateral disease, the IAR should be interpreted with caution and is not used in our clinic $(3,41-47)$. 


\section{Conclusion}

The $500 \mathrm{~Hz}$ cVEMP threshold, normalized peak-to-peak amplitude (VEMPn) and VEMP inhibition depth (VEMPid) are most valuable in separating Menière's disease (MD) from healthy control ears. The diagnostic accuracy of the $500 / 1000 \mathrm{~Hz}$ threshold and VEMPn ratio, as well as the IAR, are inferior and therefore these metrics are not recommended for evaluation of patients with clinically definite MD. 


\section{References}

1. Colebatch JG, Halmagyi GM, Skuse NF. Myogenic potentials generated by a click-evoked vestibulocollic reflex. J Neurol Neurosurg Psychiatry 1994;57:190-7.

2. Curthoys IS. A critical review of the neurophysiological evidence underlying clinical vestibular testing using sound, vibration and galvanic stimuli. Clin Neurophysiol 2010;121:132-44.

3. Rauch SD, Zhou G, Kujawa SG, et al. Vestibular evoked myogenic potentials show altered tuning in patients with Meniere's disease. Otol Neurotol 2004;25:333-8.

4. Rauch SD, Silveira MB, Zhou G, et al. Vestibular evoked myogenic potentials versus vestibular test battery in patients with Meniere's disease. Otol Neurotol 2004;25:981-6.

5. Hunter JB, Patel NS, O'Connell BP, et al. Cervical and Ocular VEMP Testing in Diagnosing Superior Semicircular Canal Dehiscence. Otolaryngol Head Neck Surg 2017;156:917-23.

6. Fife TD, Colebatch JG, Kerber KA, et al. Practice guideline: Cervical and ocular vestibular evoked myogenic potential testing: Report of the Guideline Development, Dissemination, and Implementation Subcommittee of the American Academy of Neurology. Neurology 2017;89:2288-96.

7. Noij KS, Duarte MJ, Wong K, et al. Toward Optimizing Cervical Vestibular Evoked Myogenic Potentials (cVEMP): Combining Air-Bone Gap and cVEMP Thresholds to Improve Diagnosis of Superior Canal Dehiscence. Otol Neurotol 2018;39:212-220.

8. Salviz M, Yuce T, Acar H, et al. Diagnostic value of vestibular-evoked myogenic potentials in Meniere's disease and vestibular migraine. J Vestib Res 2016;25:261-6.

9. Johnson SA, O'Beirne GA, Lin E, et al. oVEMPs and cVEMPs in patients with 'clinically certain' Meniere's disease. Acta Otolaryngol 2016;136:1029-34.

10. Zuniga MG, Janky KL, Schubert MC, et al. Can vestibular-evoked myogenic potentials help differentiate Meniere disease from vestibular migraine? Otolaryngol Head Neck Surg 2012;146:788-96.

11. Salviz M, Yuce T, Karatas A, et al. Diagnostic value of frequency-associated vestibular-evoked myogenic potential responses in Meniere's disease. Audiol Neurootol 2015;20:229-36.

12. Taylor RL, Wijewardene AA, Gibson WP, et al. The vestibular evoked-potential profile of Meniere's disease. Clin Neurophysiol 2011;122:1256-63.

13. Sandhu JS, Low R, Rea PA, et al. Altered frequency dynamics of cervical and ocular vestibular evoked myogenic potentials in patients with Meniere's disease. Otol Neurotol 2012;33:444-9.

14. Taylor RL, Zagami AS, Gibson WP, et al. Vestibular evoked myogenic potentials to sound and vibration: characteristics in vestibular migraine that enable separation from Meniere's disease. Cephalalgia 2012;32:213-25.

15. Zhu Y, McPherson J, Beatty C, et al. Cervical VEMP threshold response curve in the identification of Meniere's disease. J Am Acad Audiol 2014;25:278-88.

16. Kim-Lee Y, Ahn JH, Kim YK, et al. Tone burst vestibular evoked myogenic potentials: diagnostic criteria in patients with Meniere's disease. Acta Otolaryngol 2009;129:924-8.

17. Node M, Seo T, Miyamoto A, et al. Frequency dynamics shift of vestibular evoked myogenic potentials in patients with endolymphatic hydrops. Otol Neurotol 2005;26: 1208-13.

18. Maxwell R, Jerin C, Gurkov R. Utilisation of multi-frequency VEMPs improves diagnostic accuracy for Meniere's disease. Eur Arch Otorhinolaryngol 2017;274:85-93.

19. Egami N, Ushio M, Yamasoba T, et al. The diagnostic value of vestibular evoked myogenic potentials in patients with Meniere's disease. J Vestib Res 2013;23:249-57.

20. Inoue A, Egami N, Fujimoto C, et al. Vestibular Evoked Myogenic Potentials in Vestibular Migraine: Do They Help Differentiating From Meniere's Disease? Ann Otol Rhinol Laryngol 2016;125:931-937.

21. Lamounier P, de Souza TSA, Gobbo DA, et al. Evaluation of vestibular evoked myogenic potentials (VEMP) and electrocochleography for the diagnosis of Meniere's disease. Braz J Otorhinolaryngol 2017;83:394-403.

22. Noij KS, Herrmann BS, Rauch SD, et al. Toward Optimizing Vestibular Evoked Myogenic Potentials: Normalization Reduces the Need for Strong Neck Muscle Contraction. Audiol Neurootol 2017;22:282291.

23. Akin FW and Murnane OD. Vestibular evoked myogenic potentials: preliminary report. J Am Acad Audiol 2001;12:445-52. 
24. Akin FW, Murnane OD, Panus PC, et al. The influence of voluntary tonic EMG level on the vestibularevoked myogenic potential. J Rehabil Res Dev 2004;41:473-80.

25. Isaacson B, Murphy E, Cohen H. Does the method of sternocleidomastoid muscle activation affect the vestibular evoked myogenic potential response? J Vestib Res 2006;16:187-91.

26. Lim CL, Clouston $P$, Sheean $G$, et al. The influence of voluntary EMG activity and click intensity on the vestibular click evoked myogenic potential. Muscle Nerve 1995;18:1210-3.

27. Ochi K, Ohashi T, Nishino $\mathrm{H}$. Variance of vestibular-evoked myogenic potentials. Laryngoscope 2001;111:522-7.

28. Ochi K, Ohashi T. Age-related changes in the vestibular-evoked myogenic potentials. Otolaryngol Head Neck Surg 2003;129:655-9.

29. Basta D, Todt I, Ernst A. Characterization of age-related changes in vestibular evoked myogenic potentials. J Vestib Res 2007;17:93-8.

30. Singh NK, Kashyap RS, Supreetha L, et al. Characterization of age-related changes in sacculocolic response parameters assessed by cervical vestibular evoked myogenic potentials. Eur Arch Otorhinolaryngol 2014;271:1869-77.

31. Lopez-Escamez JA, Carey J, Chung WH, et al. Diagnostic criteria for Meniere's disease. J Vestib Res 2015;25:1-7.

32. Van Tilburg MJ, Herrmann BS, Rauch SD, et al. Normalizing cVEMPs: which method is the most effective? Ear Hear 2018. [Epub ahead of print].

33. van Tilburg MJ, Herrmann BS, Guinan JJ Jr, et al. Normalization reduces intersubject variability in cervical vestibular evoked myogenic potentials. Otol Neurotol 2014;35:e222-7.

34. Prakash SR, Herrmann BS, Milojcic R, et al. Evaluating Inhibition of Motoneuron Firing From Electromyogram Data to Assess Vestibular Output Using Vestibular Evoked Myogenic Potentials. Ear Hear 2015;36:591-604.

35. Noij KS, van Tilburg MJ, Herrmann BS, et al. Toward Optimizing VEMP: Calculating VEMP Inhibition Depth With a Generic Template. Ear Hear 2018;39(6):1199-206.

36. Murofushi T, Nakahara H, Yoshimura E, et al. Association of air-conducted sound oVEMP findings with cVEMP and caloric test findings in patients with unilateral peripheral vestibular disorders. Acta Otolaryngol 2011;131:945-50.

37. Taylor RL, Bradshaw AP, Halmagyi GM, et al. Tuning characteristics of ocular and cervical vestibular evoked myogenic potentials in intact and dehiscent ears. Audiol Neurootol 2012;17:207-18.

38. van Tilburg MJ, Herrmann BS, Guinan JJ Jr, et al. Serial cVEMP Testing is Sensitive to Disease Progression in Meniere Patients. Otol Neurotol 2016;37:1614-1619.

39. Timmer FC, Zhou G, Guinan JJ, et al. Vestibular evoked myogenic potential (VEMP) in patients with Ménière's disease with drop attacks. Laryngoscope 2006 May;116:776-9.

40. Young YH, Huang TW, Cheng PW. Assessing the stage of Meniere's disease using vestibular evoked myogenic potentials. Arch Otolaryngol Head Neck Surg 2003;129:815-8.

41. Paparella MM and Griebie MS. Bilaterality of Meniere's disease. Acta Otolaryngol 1984;97:233-7.

42. Stahle J, Friberg U, Svedberg A. Long-term progression of Meniere's disease. Am J Otol 1989;10:170-3.

43. Yazawa $Y$, Kitahara M. Bilateral endolymphatic hydrops in Meniere's disease: review of temporal bone autopsies. Ann Otol Rhinol Laryngol 1990;99:524-8.

44. Tsuji K, Velázquez-Villaseñor L, Rauch SD, et al. Temporal bone studies of the human peripheral vestibular system. Meniere's disease. Ann Otol Rhinol Laryngol Suppl 2000;181:26-31.

45. House JW, Doherty JK, Fisher LM, et al. Meniere's disease: prevalence of contralateral ear involvement. Otol Neurotol 2006;27:355-61.

46. Chaves AG, Boari L, Lei Munhoz MS. The outcome of patients with Menieres disease. Braz J Otorhinolaryngol 2007;73:346-50.

47. Shojaku H, Watanabe Y, Yagi T, et al. Changes in the characteristics of definite Meniere's disease over time in Japan: a long-term survey by the Peripheral Vestibular Disorder Research Committee of Japan, formerly the Meniere's Disease Research Committee of Japan. Acta Otolaryngol 2009;129:155-60. 




\section{Chapter 4.2}

\section{Predicting development of bilateral Menière's disease based on cVEMP threshold and tuning}




\section{Abstract}

\section{Objective}

To investigate if cVEMP is predictive for developing bilateral Menière's disease (MD).

\section{Study design}

Retrospective cohort study

\section{Setting}

Tertiary care center

\section{Patients}

Records of seventy-one patients previously diagnosed with unilateral Menière's disease at our institution who underwent cVEMP testing between 2002 and 2011 were screened.

\section{Intervention}

Patients were contacted to answer a questionnaire to identify which patients had developed bilateral disease. Based on questionnaires and medical charts, 49 patients with a follow-up time of at least 5 years were included. The 49 originally asymptomatic ears are referred to as "study ears". Previously reported cVEMP criteria (original criteria) applied to study-ear cVEMPs separated them into Menière-like and normal-like groups.

\section{Main outcome measure}

The main purpose was to determine if previously obtained cVEMP thresholds and tuning ratios of unilateral MD patients could predict who develops bilateral disease.

\section{Results}

From the 49 included patients, 12 developed bilateral disease (24.5\%). The study ears characterized by original cVEMP criteria as Menière-like were significantly more likely to develop bilateral disease compared to the normal-like study ears. The original criteria predicted development of bilateral disease with a positive predictive value (PPV) and negative predictive value (NPV) of $58.3 \%$ and $86.5 \%$ respectively. ROC curves were used to revise CVEMP criteria for predicting the progression to bilateral disease. A revised criterion combining three CVEMP metrics, reached a PPV and NPV of $85.7 \%$ and 93.7\%.

\section{Conclusion}

cVEMP threshold and tuning in unilateral MD patients are predictive of which patients will develop bilateral disease. 


\section{Introduction}

Patients with Menière's disease (MD) suffer from a combination of symptoms, including vertigo attacks, hearing loss, tinnitus and aural fullness (1). Temporal bone studies have revealed the presence of saccular endolymphatic hydrops in MD patients (2). However, it is difficult to determine the presence of endolymphatic hydrops in vivo and its presence does not necessarily indicate MD (3). The only available test that can measure saccular function is the cervical vestibular evoked myogenic potential (cVEMP) and multiple studies have shown that symptomatic MD ears have lower CVEMP amplitudes, higher thresholds and altered frequency tuning compared to healthy controls (4-7). To evoke a cVEMP, the saccule is acoustically activated, resulting in an ipsilateral inhibition of the contracted sternocleidomastoid muscle (SCM) that is measured with electromyography (EMG) $(8,9)$.

Patients with unilateral MD have an about 25 to $35 \%$ risk of developing bilateral disease. At present no measures exist to predict which patients will develop bilateral disease, even though previous reports have noted differences in CVEMP metrics between asymptomatic ears of patients with unilateral MD and control ears (7,10-17). Some of these asymptomatic ears showed cVEMP amplitudes and thresholds that were more similar to the symptomatic MD ears than ears of healthy controls $(4,7,10)$. It has been hypothesized that the asymptomatic ears with "Menière-like" cVEMP outcomes might be the ears developing disease $(4,7,10)$. Lin et al. studied temporal bones of unilateral MD patients and found saccular hydrops in 35\% of asymptomatic ears. In a separate group of unilateral MD patients with cVEMPs, $27 \%$ of asymptomatic ears showed a "Menière-like" cVEMP response, based on cVEMP threshold and tuning (10). Although this is not direct proof, it does suggest a relationship and the authors concluded that the similarity in these percentages supports the hypothesis that saccular hydrops might produce changes in cVEMPs that precede symptoms in bilateral MD patients (10). To our knowledge, no study exists that aims to test this hypothesis. In the current study, patients with unilateral MD, who underwent a CVEMP at our institution between 2002 and 2011 were contacted to determine which patients had developed bilateral disease. We then investigated if the previously-obtained cVEMPs and the previously-reported criteria for "Menière-like" cVEMPs could predict which patients had become bilaterally symptomatic (10).

\section{Methods}

\section{Methods overview}

This study includes patients who were diagnosed with unilateral MD $\geq 5$ years ago. All physiologic tests analyzed in this study were performed after initial diagnosis, at least 5 
years before follow-up assessment. Based on the symptoms at the time of cVEMP testing, ears were divided into "symptomatic" and "asymptomatic" for MD. The originally asymptomatic ears are the ears of interest in this study and will be referred to as the "study ears". In the patient follow-up, assessment of whether a study ear had become symptomatic was done based on questionnaire responses and medical records. No physiologic tests were done for the follow-up assessment.

\section{Subjects}

Records of patients with Menière's disease who were previously included in studies at our institution were screened for inclusion in the current study (Figure 4.2.1) $(7,10,18)$. Patients who met the following criteria were identified: 1) A diagnosis of unilateral Menière's disease at the time of cVEMP testing and 2) cVEMP testing that was done between 2002 and 2011. After the exclusion of doubles and of one patient with superior semicircular canal dehiscence on the same side as the symptomatic Menière ear, seventy-one patients remained. Patients with an air-bone gap ( $A B G$ ) of $\geq 15 \mathrm{~dB}$ at one or more frequencies at the time of the CVEMP were excluded $(n=5)$. The remaining 66 patients were contacted by phone, email or mail to answer a questionnaire about the study ear. Patients were asked if they had suffered from vertigo attacks plus hearing loss and aural fullness and/or tinnitus in the study ear. If patients suffered from these symptoms they were asked when the symptoms first occurred. Thirty-six patients answered the questionnaire. Medical charts from the remaining 30 patients were reviewed to evaluate if they had developed bilateral disease. Seventeen out of these 30 patients were excluded because the time between their diagnosis and last visit was $<5$ yrs. In total, 49 patients could be included for analyses (Figure 4.2.1). This study was approved by the Human Studies Committee of the Massachusetts Eye and Ear (\#13-097H, PI: S.D. Rauch).

\section{Bilateral disease}

At follow-up assessment, patients who suffered from vertigo attacks, plus hearing loss, tinnitus and/or aural fullness in the study ear were considered to have developed bilateral disease. Depending on the availability of an audiogram obtained after the start of symptoms in the study ear, these ears could be further separated into probable MD or definite MD according to the Lopez-Escamez et al. 2015 criteria (1). 


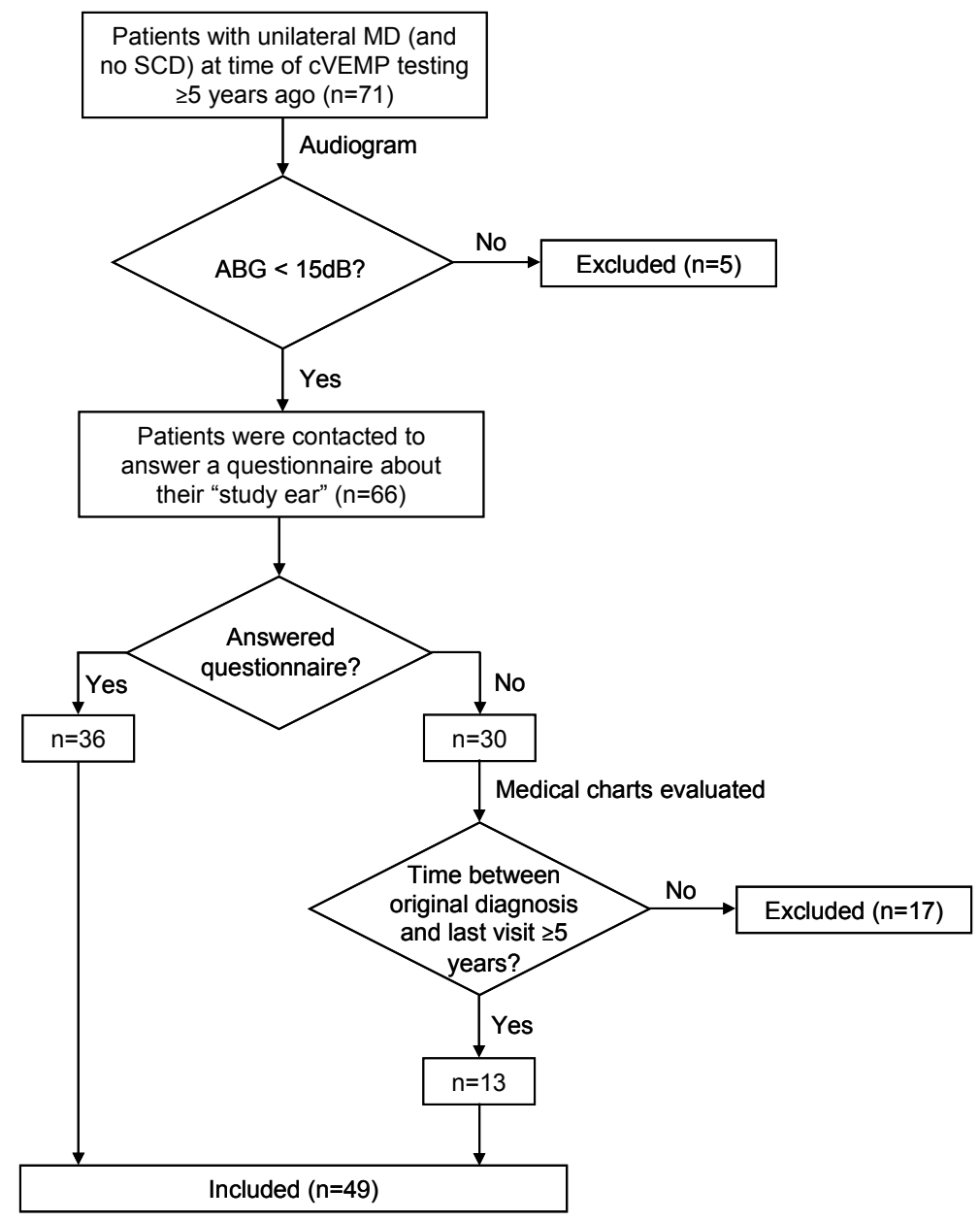

Figure 4.2.1 Flowchart displaying patient inclusion criteria. Of the 71 patients identified for screening, 49 patients could be included. The "study ear" is the ear that was asymptomatic during cVEMP testing $\geq 5$ years ago. MD indicates Menière's disease; SCD indicates semicircular canal dehiscence syndrome; ABG indicates air-bone gap.

\section{Audiometry}

At the time of cVEMP testing, all subjects also underwent audiometric testing with pure tone air- and bone-conduction threshold measurements at octave frequencies from 250 to $4000 \mathrm{~Hz}$. For each frequency, the ABG was calculated by subtracting the boneconduction threshold from the corresponding air-conduction threshold. Five patients had an $A B G$ of $\geq 15 \mathrm{~dB}$ at one or more frequencies at the time of the CVEMP and were 
excluded from this study, because this ABG might indicate the presence of middle ear pathology, which could increase cVEMP thresholds.

\section{CVEMP}

All patients underwent bilateral cVEMP testing using our custom-programmed evoked potential system that has been described previously $(7,18)$. cVEMPs were obtained with the patient sitting up straight and turning their head away from the stimulated ear, thereby contracting the ipsilateral SCM. EMG activity was recorded using five surface electrodes: a non-inverting electrode on the middle belly of each SCM, an inverting electrode on the muscle tendon just above the clavicle, and a ground electrode on the midline forehead. Patients contracted their SCM to produce more than $65 \mu \mathrm{V}$ root mean square (rms) EMG during recording. EMG activity was amplified, bandpass filtered between 10 and $2000 \mathrm{~Hz}$ and sampled at $50 \mathrm{kHz}$. Tone burst stimuli were presented at 250, 500 and $1000 \mathrm{~Hz}$ (Blackman gating function, two cycle rise and fall times with no plateau) in all patients, and 34 patients were also tested using $750 \mathrm{~Hz}$. Tone bursts were monaurally presented at a repetition rate of 13 bursts/s, using circumaural headphones (Telephonics TDH-49). For each recording, at least $100 \mathrm{cVEMP}$ responses were obtained and averaged. Tone bursts were presented, starting at $133 \mathrm{~dB}$ peSPL at $250 \mathrm{~Hz}$ and $123 \mathrm{~dB}$ peSPL for 500,750 and $1000 \mathrm{~Hz}$ (these sound levels are equivalent to $90 \mathrm{~dB} \mathrm{HL}$ ) followed by sound level decreases in steps of $10 \mathrm{~dB}$ until no response could be distinguished from residual noise. To determine threshold, sound levels were then raised by $5 \mathrm{~dB}$. If no CVEMP was present at our equipment limit (143 dB peSPL at $250 \mathrm{~Hz}$ and $133 \mathrm{~dB}$ peSPL at 500, 750 and $1000 \mathrm{~Hz}$ ), threshold was defined as $10 \mathrm{~dB}$ higher than this limit. Finally, the $500 / 1000 \mathrm{~Hz}$ threshold ratio was calculated to evaluate tuning.

\section{Original cVEMP criteria}

The cVEMPs of the study ears were divided into 2 groups based on threshold and tuning criteria described by Lin et al. 2006: Normal-like or Menière-like. For the threshold criterion, ears with a cVEMP threshold below the study ears' mean (as reported by Lin et al.) at $\geq 2$ frequencies were considered "normal-like", while the other ears were "Menière-like". For the tuning criterion, ears with a cVEMP threshold of $500 \leq 1000 \mathrm{~Hz}$ were "normal-like", while cVEMP thresholds $500>1000 \mathrm{~Hz}$ were "Menière-like". As in the Lin et al. study, only ears that were considered "Menière-like" according to both the threshold and the tuning criteria were categorized as "Menièrelike" (10). 


\section{Data analysis}

Which patients developed bilateral disease was determined using questionnaires and medical chart data, as described previously. Follow-up times were calculated and Kaplan-Meier curves indicating time to developing bilateral disease (the event), were created for both the "normal-like" and "Menière-like" groups. The difference between the curves was analyzed using a log-rank test. A $2 \times 2$ table was created to indicate which patients were correctly identified by the original criterion as becoming bilaterally affected and the positive predictive value, negative predictive value, sensitivity and specificity of these original criteria were calculated from these data (10).

The available audiometric and CVEMP data were analyzed further using factorial analyses of variance (ANOVA). Audiometric bone-conduction thresholds and CVEMP thresholds were compared between the study ears that remained asymptomatic and the ears that became symptomatic. Group and frequency were considered fixed factors and patient was considered a random factor. Post hoc comparisons were performed comparing the two groups at each frequency. Independent-sample $t$ tests were used to compare the $500 / 1000 \mathrm{~Hz}$ cVEMP threshold ratios for these groups.

Pearson correlation coefficients were computed to evaluate the relationship between cVEMP thresholds and the time from cVEMP testing to development of bilateral disease.

Besides investigating the ability of the previously described original criteria to predict which patients develop bilateral disease, we also investigated the predictive value of cVEMP thresholds and 500/1000 Hz cVEMP threshold tuning ratios post hoc using ROC curves. These ROC curves were used to derive "revised criteria" for predicting which patients would develop bilateral disease.

Statistical analyses were performed using SPSS (version 24.0; Chicago, IL). A $p$ value of $<0.05$ was considered statistically significant.

\section{Results}

\section{Patient characteristics}

Forty-nine patients were included (mean age: 49.2 years, age range: 26-74 years; 29 women). All patients had unilateral MD at the time of cVEMP testing (24 right ears, 25 left ears). From the 49 included patients, 12 (24.5\%) developed bilateral disease according to the criteria described in the Methods section. Of these 12 ears, 6 could be categorized as definite MD and 6 as probable MD according to the Lopez-Escamez et al. criteria (1). The time between the MD diagnosis of the first and second ear varied from 24 to 288 months (average: 136 months). The follow-up time in patients who did not develop bilateral disease varied from 60 to 360 months (average: 176 months). Audiometric bone-conduction thresholds did not significantly differ between the study 
ears that developed disease and the ears that remained asymptomatic ( $p=0.263$; data not shown).

\section{The original cVEMP prediction}

Using the Lin et al. cVEMP criteria (the original criteria described in Methods), 12 study ears were considered Menière-like, while 37 study ears were considered normal-like. From the Menière-like group, 7/12 ears actually became symptomatic and from the normal-like group 5/37 ears became symptomatic (Table 4.2.1). The Kaplan-Meier curves of the normal-like and Menière-like group differed significantly (Hazard ratio = 4.46 [95\% Cl: 1.41-14.14]; $p=0.005$; Figure 4.2.2), indicating that the Menière-like group was more likely to develop bilateral disease than the normal-like group. To calculate the positive predictive value (PPV) and negative predictive value (NPV), a prevalence of bilateral disease of $24.5 \%$ was assumed, based on our own data. The PPV and NPV of the original criteria to detect development of bilateral disease were $58.3 \%$ and $86.5 \%$ respectively (Table 4.2 .1 ).

Table 4.2.1 Table indicating the relationship between whether patients developed bilateral disease and their categorization as Menière-like and normal-like based on the original criteria. The percentages indicate the sensitivity, specificity and predictive values of this original categorization. This study group's own prevalence (24.5\%) was used for these calculations.

\begin{tabular}{|c|c|c|c|c|}
\hline & $\begin{array}{l}\text { Developed } \\
\text { bilateral disease }\end{array}$ & $\begin{array}{l}\text { Did not develop } \\
\text { bilateral disease }\end{array}$ & Total & \\
\hline Menière-like cVEMP & 7 & 5 & 12 & Positive predictive value $=\mathbf{5 8 . 3} \%$ \\
\hline \multirow[t]{3}{*}{ Normal-like cVEMP } & 5 & 32 & 37 & Negative predictive value $=86.5 \%$ \\
\hline & 12 & 37 & 49 & \\
\hline & Sensitivity $=58.3 \%$ & Specificity $=86.5 \%$ & & \\
\hline
\end{tabular}

\section{cVEMP thresholds and tuning}

Figure 4.2.3 plots the mean cVEMP thresholds of each study ear group with comparison to the cVEMP thresholds of the non-study ears (the original symptomatic ear of the unilateral Menière's diagnosis). The study ears that remained asymptomatic had significantly lower cVEMP thresholds at 250,500 and $750 \mathrm{~Hz}$ compared to the ears that became symptomatic ( $p=0.001, p<0.001$ and $p=0.002$, respectively), while at $1000 \mathrm{~Hz}$ there was no significant difference between the two groups ( $p=0.495$; Figure 4.2.3). The study ears that developed disease had CVEMP thresholds similar to the originally symptomatic ears (shaded area Figure 4.2.3). 


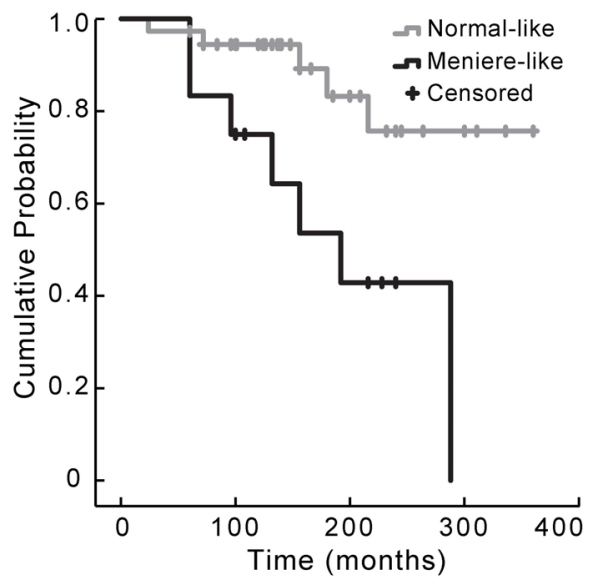

Figure 4.2.2 Kaplan-Meier curve indicating cumulative probability of remaining asymptomatic plotted against time from original diagnosis to development of bilateral disease (the event) or end of follow-up. The grey line represents the normal-like group and the black line represents the Menière-like group, categorized based on the original criteria. Plusses indicate the end of follow-up of a patient (censored) who did not develop bilateral disease up to that moment. For these patients, no further follow-up data are available, either because of loss of follow-up or because that moment was the end of this study. These curves show that the time to develop bilateral disease varied from 2 to 24 years. The Hazard ratio was calculated by dividing the slope of the MD-like ears by the slope of the normal-like ears using SPSS (HR = 4.46).

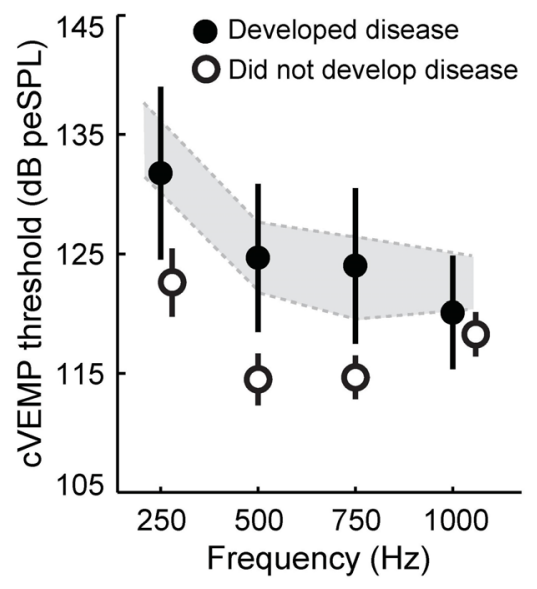

Figure 4.2.3 Average cVEMP thresholds of study ears that developed disease (black circles) compared to study ears that did not develop disease (open circles). Error bars indicate $95 \%$ confidence intervals. The shaded area indicates the $95 \%$ confidence intervals of the cVEMP thresholds of the originally symptomatic MD ears when all patients still had unilateral MD. The study ears that developed bilateral disease had CVEMP thresholds similar to the originally symptomatic ears. 
A difference in tuning was also observed between the ears that remained asymptomatic and the ones that developed disease. Of the ears that developed disease, $75.0 \%$ had a lower threshold at $1000 \mathrm{~Hz}$ than at $500 \mathrm{~Hz}$, while this occurred in only $18.9 \%$ of the ears that did not develop disease (Figure 4.2.4). Furthermore, $500 / 1000 \mathrm{~Hz}$ cVEMP threshold ratios were significantly higher in the newly symptomatic ears $(\mu=1.038,95 \% \mathrm{Cl}: 1.011-1.065)$ compared to the ears that remained asymptomatic ( $\mu=0.969,95 \% \mathrm{Cl}: 0.951-0.987 ; p<0.001)$. No significant age difference at the time of cVEMP testing was found between the two groups (average age: 52.83 years versus 49.27 years; $p=0.421$ ). Furthermore, there was no significant difference in age between the true positive, true negative, false positive and false negative groups $(F=0.245, p=0.864)$. There was no significant relationship between the time from cVEMP to the development of bilateral disease and the CVEMP threshold at $250(\rho=-0.046, p=0.892), 500(\rho=0.218, p=0.519), 750(\rho=0.336, p=0.342)$ and $1000 \mathrm{~Hz}(\rho=0.280, p=0.405)$.

\section{Study ears}

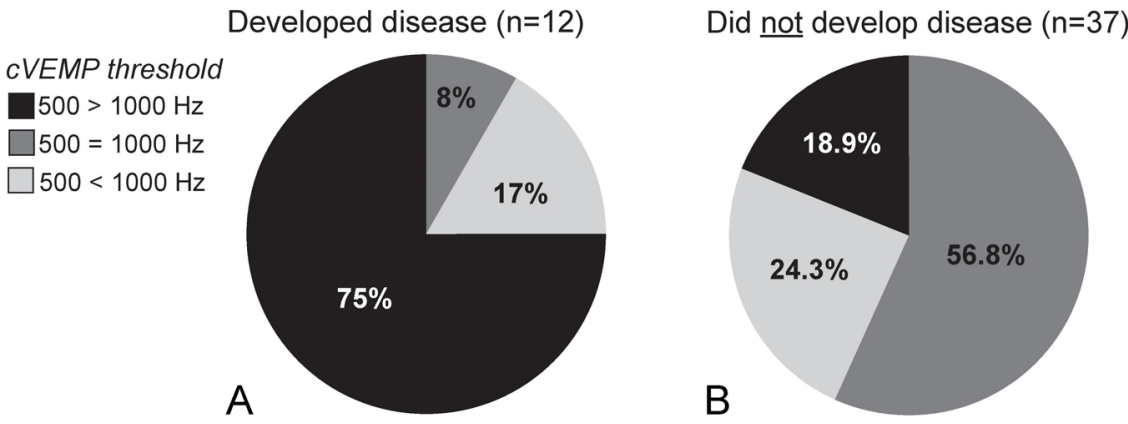

Figure 4.2.4 Distribution of cVEMP threshold tuning ( $500 \mathrm{~Hz}$ cVEMP thresholds relative to the $1000 \mathrm{~Hz}$ ) of the former asymptomatic ears. In the former asymptomatic ears that developed disease, 75\% of the $500 \mathrm{~Hz}$ thresholds were higher than the $1000 \mathrm{~Hz}$ in these ears (A), while in the ears that remained asymptomatic, only $18.9 \%$ of ears had $500 \mathrm{~Hz}$ thresholds that were higher than the $1000 \mathrm{~Hz}$ thresholds (B).

\section{Revised cVEMP prediction criteria}

The data obtained from our follow-up assessment were analyzed post hoc to develop revised criteria for predicting which patients develop bilateral disease using CVEMP thresholds and tuning ratios. Based on ROC curve analysis (see Appendix 4.2.1), if only one metric was used, the $500 \mathrm{~Hz}$ cVEMP threshold provided the best combination of sensitivity (75\%) and specificity (83.8\%). The corresponding cutoff value (a $500 \mathrm{~Hz}$ cVEMP threshold of $>121 \mathrm{~dB}$ peSPL) could predict development of bilateral disease with a PPV of $60 \%$ and a NPV of $91.2 \%$. ROC areas under the curve (AUC) indicated that 
combining the three best individual cVEMP predictors (the $500 \mathrm{~Hz}$ threshold, $750 \mathrm{~Hz}$ threshold and $500 / 1000 \mathrm{~Hz}$ tuning ratio), improved the prediction of developing bilateral disease (Appendix 4.2.1). The combination of the three best metrics $(500 \mathrm{~Hz}$ threshold, $750 \mathrm{~Hz}$ threshold and $500 / 1000 \mathrm{~Hz}$ tuning ratio) provided a sensitivity of $80 \%$ with a specificity of $95.8 \%$, corresponding to a PPV of $85.7 \%$ and NPV of $93.7 \%$.

\section{Discussion}

Patients with unilateral Menière's disease have about a 25 to 35\% risk of developing bilateral disease $(7,10-17)$. We investigated whether cVEMP thresholds and tuning could predict which unilateral MD patients would develop bilateral disease. The original cVEMP criteria suggested by Lin et al. provided a PPV of $58.3 \%$ and NPV of $86.5 \%$ for the development of bilateral MD (10). That is, previously asymptomatic ears with a "Menière-like" cVEMP had a 58.3\% chance of developing bilateral disease, while ears with a "normal-like" cVEMP had only a $13.5 \%$ chance of developing bilateral disease. This finding validates the Lin et al. hypothesis that cVEMP thresholds and tuning can be used to predict the chance that a unilateral MD patient might develop bilateral disease. In addition, our findings indicate that pre-symptomatic changes were present in the saccule on the non-symptomatic side of a quarter of patients with unilateral Menière's disease $(7,10)$.

The accuracy of predicting the development of bilateral disease was better if CVEMP prediction criteria were revised using ROC analysis of CVEMP thresholds and $500 / 1000 \mathrm{~Hz}$ threshold ratios. The best individual metric (the $500 \mathrm{~Hz}$ cVEMP threshold) provided a PPV of $60 \%$ and NPV of $91.2 \%$. Prediction ability improved further with a combination of the best three metrics $(500 \mathrm{~Hz}$ threshold, $750 \mathrm{~Hz}$ threshold and $500 / 1000 \mathrm{~Hz}$ tuning ratio - see formula in the Appendix 4.2.1) that yielded a PPV of $85.7 \%$ and NPV of $93.7 \%$. These revised criteria are superior to the originally suggested criteria for predicting which MD patients develop bilateral disease.

It should be noted that the predictive values in this study were calculated using the cVEMP methods at our institution. This includes multi-frequency cVEMP testing, which is not standard practice in most clinics. This study emphasizes the value of multifrequency testing to predict which unilateral MD patients will develop bilateral disease. Although the cVEMP threshold and tuning ratio are useful in predicting who develops bilateral MD disease, they convey little information about the timing or severity of the disease. Only small, and not statistically significant, correlations were found between cVEMP threshold at any frequency and the time from cVEMP to the development of bilateral disease. Differences in cVEMP threshold between patients who developed probable versus definite MD were also minimal and not statistically significant (data not shown). 
In contrast to the CVEMP, audiometric bone-conduction thresholds did not show predictive value for the development of bilateral disease. For the study ears, boneconduction thresholds were not statistically significantly different between ears that became symptomatic and ears that remained asymptomatic. It is possible that the saccule is more sensitive than the cochlea to early pathologic changes in Menière's disease. The lack of vestibular symptoms in these patients could be explained by the need for a certain amount of saccular damage before symptoms arise. Another explanation may be that the vestibular system is more amenable to central adaptation than the auditory system.

In the future, the predictive value of cVEMP may be used to inform patients with unilateral MD of their likelihood to develop bilateral disease and to evaluate the effect of potential preventive measures. It remains to be seen how effective the CVEMP is at predicting MD in patients with vestibular symptoms who have not yet been assigned a diagnosis.

\section{Comparison with previous studies}

Although previous studies on the prevalence of bilateral Menière's disease found percentages ranging from 9 to 50\%, most studies reported bilateral disease in about a quarter to a third of patients, which is similar to the $24.5 \%$ found in our cohort (11$13,15-17,19-21)$. The time between diagnosis of the first and second ear varied widely in the current study, with an average of 11.3 years. This is in contrast to a study by Thomas and Harrison and Rosenberg et al., who reported that most patients who develop bilateral disease do so within 2 years after diagnosis of their first ear $(11,20)$. Stahle et al. found that after 10 years, about $22 \%$ of unilateral MD patients developed bilateral disease and that this percentage kept growing with $47 \%$ developing bilateral disease after 30 years (19).

As in the current study, previous reports have found a shift in frequency tuning in symptomatic MD ears compared to healthy ears, with most symptomatic ears having higher CVEMP thresholds at $500 \mathrm{~Hz}$ than at $1000 \mathrm{~Hz}(5,22)$. To the best of our knowledge, the current study is the first to show that this tuning shift is predictive for the development of bilateral disease.

\section{Limitations}

Due to the retrospective nature of this study there is a risk for recall bias. Additionally, follow-up times differed between patients. Although follow-up times in this study were relatively long, the possibility that some of the ears categorized as "remaining asymptomatic" would eventually develop bilateral disease cannot be excluded. It would be ideal to perform a prospective, long-term follow-up study to investigate how well cVEMPs predict bilateral disease over many decades. Especially the revised cVEMP criteria require prospective validation to determine if they can reliably be applied. In 
addition, cVEMP thresholds are known to increase with age (23). Although no significant effect of age was found amongst the true positives, true negatives, false positives and false negatives, these groups were small. To assess if the criteria should be refined for different age groups, a larger study including a sufficient number of patients in each age group would be appropriate.

\section{Conclusion}

The CVEMP is sensitive to pre-symptomatic changes in the saccule of patients with unilateral MD and can be used to predict which patients will develop bilateral disease. 


\section{References}

1. Lopez-Escamez JA, Carey J, Chung WH, et al. Diagnostic criteria for Menière's disease. J Vestib Res 2015; 25:1-7.

2. Rauch SD, Merchant SN, Thedinger BA. Menière's syndrome and endolymphatic hydrops. Double-blind temporal bone study. Ann Otol Rhinol Laryngol 1989;98:873-83.

3. Merchant SN, Adams JC, Nadol JB Jr. Pathophysiology of Menière's syndrome: are symptoms caused by endolymphatic hydrops? Otol Neurotol 2005;26:74-81.

4. Noij KS, Herrmann BS, Guinan JJ Jr., et al. Cervical Vestibular Evoked Myogenic Potentials in Menière's disease: A comparison of response metrics. Otol Neurotol 2019;40:e215-e224.

5. Salviz M, Yuce $T$, Acar $H$, et al. Diagnostic value of vestibular-evoked myogenic potentials in Menière's disease and vestibular migraine. J Vestib Res 2016;25:261-6.

6. Taylor RL, Zagami AS, Gibson WP, et al. Vestibular evoked myogenic potentials to sound and vibration: characteristics in vestibular migraine that enable separation from Menière's disease. Cephalalgia 2012;32:213-25.

7. Rauch SD, Zhou G, Kujawa SG, et al. Vestibular evoked myogenic potentials show altered tuning in patients with Menière's disease. Otol Neurotol 2004;25:333-8.

8. Colebatch JG, Halmagyi GM, Skuse NF. Myogenic potentials generated by a click-evoked vestibulocollic reflex. J Neurol Neurosurg Psychiatry 1994;57:190-7.

9. Curthoys IS. A critical review of the neurophysiological evidence underlying clinical vestibular testing using sound, vibration and galvanic stimuli. Clin Neurophysiol 2010;121:132-44.

10. Lin MY, Timmer FC, Oriel BS, et al. Vestibular evoked myogenic potentials (VEMP) can detect asymptomatic saccular hydrops. Laryngoscope 2006;116:987-92.

11. Thomas K, Harrison MS. Long-term follow up of 610 cases of Menière's disease. Proc $R$ Soc Med 1971;64:853-7.

12. Paparella MM, Griebie MS. Bilaterality of Menière's disease. Acta Otolaryngol 1984;97:233-7.

13. Wladislavosky-Waserman P, Facer GW, Mokri B, et al. Menière's disease: a 30-year epidemiologic and clinical study in Rochester, Mn, 1951-1980. Laryngoscope 1984;94:1098-102.

14. Kitahara M, Matsubara $\mathrm{H}$, Takeda $\mathrm{T}$, et al. Bilateral Menière's disease. Adv Otorhinolaryngol 1979;25:117-21.

15. Tsuji K, Velázquez-Villaseñor L, Rauch SD, et al. Temporal bone studies of the human peripheral vestibular system. Menière's disease. Ann Otol Rhinol Laryngol Suppl 2000;181:26-31.

16. House JW, Doherty JK, Fisher LM, et al. Menière's disease: prevalence of contralateral ear involvement. Otol Neurotol 2006;27:355-61.

17. Chaves AG, Boari L, Lei Munhoz MS. The outcome of patients with Menière's disease. Braz J Otorhinolaryngol 2007;73:346-50.

18. van Tilburg MJ, Herrmann BS, Guinan JJ Jr, et al. Serial cVEMP Testing is Sensitive to Disease Progression in Menière Patients. Otol Neurotol 2016;37:1614-1619.

19. Stahle J, Friberg U, Svedberg A. Long-term progression of Menière's disease. Am J Otol 1989;10:170-3.

20. Rosenberg $\mathrm{S}$, Silverstein $\mathrm{H}$, Flanzer J, et al. Bilateral Menière's disease in surgical versus nonsurgical patients. Am J Otol 1991;12:336-40.

21. Shojaku H, Watanabe $Y$, Yagi $T$ et al. Changes in the characteristics of definite Menière's disease over time in Japan: a long-term survey by the Peripheral Vestibular Disorder Research Committee of Japan, formerly the Menière's Disease Research Committee of Japan. Acta Otolaryngol 2009;129:155-60.

22. Maxwell R, Jerin C, Gürkov R. Utilisation of multi-frequency VEMPs improves diagnostic accuracy for Menière's disease. Eur Arch Otorhinolaryngol 2017;274:85-93

23. Welgampola MS, Colebatch JG. Vestibulocollic reflexes: normal values and the effect of age. Clin Neurophysiol 2001;112:1971-9. 


\section{Appendix 4.2.1}

ROC curves were generated post hoc to assess the ability of CVEMP thresholds and tuning ratio to predict which patients develop bilateral disease (see Figure A4.2.1). The three best individual CVEMP predictors for development of bilateral disease were the $500 \mathrm{~Hz}$ threshold, $750 \mathrm{~Hz}$ threshold and $500 / 1000 \mathrm{~Hz}$ tuning ratio based on ROC areas under the curve (AUC). We explored if a combination of these metrics would improve the predictability of developing bilateral disease by combining them using a logistic regression model. The optimal combination of the original metrics was combined into a new metric, beta $(\beta)$, for each subject that was calculated using the following formula:

$$
\begin{gathered}
\beta=-58.850+(500 \mathrm{~Hz} \text { threshold } *-0.167)+(750 \mathrm{~Hz} \text { threshold } * 0.303)+(500 / 1000 \mathrm{~Hz} \\
\text { threshold ratio * 42.456) }
\end{gathered}
$$

The $\beta$ value was used to create an ROC curve for the combination of metrics, achieving an AUC of 0.900 . The corresponding optimal sensitivity, specificity and cutoff value were $80 \%, 95.8 \%$ and -0.149 respectively.

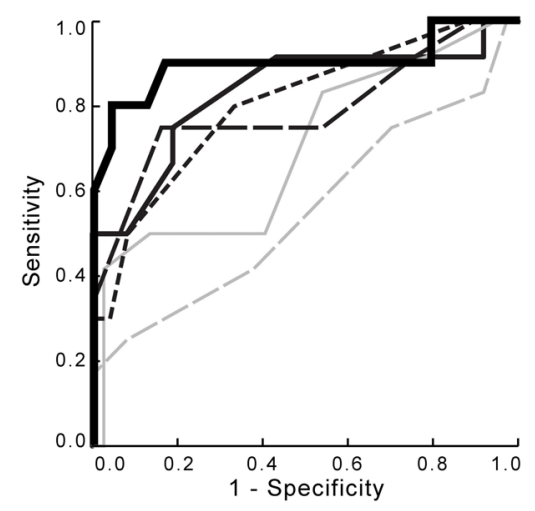

$\begin{array}{lcc} & \text { Area under } \\ \text { Source of the curve } & \text { the curve (AUC) } & 95 \% \mathrm{Cl} \\ -250 \mathrm{~Hz} & 0.697 & (0.515-0.879) \\ --500 \mathrm{~Hz} & 0.789 & (0.614-0.964) \\ --750 \mathrm{~Hz} & 0.804 & (0.637-0.972) \\ --1000 \mathrm{~Hz} & 0.553 & (0.346-0.760) \\ -500 / 1000 \mathrm{~Hz} \text { ratio } & 0.833 & (0.679-0.988) \\ -500 \mathrm{~Hz}+750 \mathrm{~Hz} & 0.900 & (0.750-1.000) \\ +500 / 1000 \mathrm{ratio} & & \end{array}$

Figure A4.2.1 Receiver operating characteristic (ROC) curves displaying the ability of various cVEMP metrics to predict who will develop bilateral Menière's disease (MD). Each thin line indicates the sensitivity for developing bilateral disease (the true-positive rate) versus the false positive rate (1-specificity) for $250 \mathrm{~Hz}, 500 \mathrm{~Hz}, 750$ and 1000 threshold and 500/1000 Hz threshold ratio. The thick line represents the ROC created by a combination of the best three metrics ( $500 \mathrm{~Hz}$ threshold, $750 \mathrm{~Hz}$ threshold and $500 / 1000 \mathrm{~Hz}$ threshold ratio). The area under the curve $(A \cup C)$ and $95 \%$ confidence interval $(95 \% \mathrm{CI})$ of each AUC are presented. 



\section{Chapter}

Discussion 



\section{Discussion}

The cervical vestibular evoked myogenic potential (cVEMP) is a promising tool to evaluate saccular (dys)function. However, there is currently no consensus on how the cVEMP should be used in clinic. Many factors other than saccular function affect the cVEMP response (e.g. muscle contraction and age) and increase the response variability. The cVEMP can only reliably be used to assess saccular function if other factors contributing to its' variability are controlled. It is crucial to use refined methods that accurately correct for these other non-saccular variables when using the cVEMP in a clinical population. In this thesis, a variety of methods to do this were studied and are discussed below.

\section{VEMP inhibition depth}

One metric developed to better reflect saccular function in the CVEMP response is the VEMP inhibition depth (VEMPid). VEMPid was developed using computational modeling to estimate the inhibition of sternocleidomastoid muscle (SCM) activity caused by saccular activation. This metric uses a template correlation method, resembling a matched filter for the detection of the CVEMP in noise and requires the use of a "template". In the original VEMPid method, the template was obtained from the subject being tested. The use of this subject-specific template becomes problematic when testing patients with vestibular pathology who may not have robust CVEMP responses. In theory, it would be appropriate to obtain a template from the other "healthy" ear. However, bilateral vestibular disease is not uncommon and its presence would invalidate this option. Alternatively, a generic template comprised of responses from healthy subjects could be used. The use of a generic template to compute VEMPid was compared to using the subject-specific template and it proved to be a reliable alternative. The VEMPids calculated with the generic versus subject-specific templates correlated strongly (correlation coefficient of 0.989) and no significant differences in VEMPid were found between the two templates. This provided a step in the direction for VEMPid use in a patient population. Before this technique can be used in clinic, the use of the generic template to calculate VEMPid should be tested in a patient population. Another advantage of VEMPid is that it averages to zero when no stimulus, and therefore no response, is present. In contrast, the normalized peak-to-peak amplitude does not average to zero in the same situation, indicating the presence of a noise floor, which makes it more difficult to determine when a response is present or absent. The VEMPid reaching zero when no response is present is an advantage that could aid in threshold determination. Potentially, the VEMPid might also be helpful in determining when to stop recording. Observing the accumulating VEMPid during data acquisition provides a guide in determining when enough data has been collected. In summary, the VEMPid has the potential to more accurately reflect saccular function 
than currently used methods. Clinically, this would decrease cVEMP variability allowing for a more accurate comparison of saccular (dys)function between patient groups (Chapter 2.1).

\section{Determining preferred muscle contraction range for normalization of cVEMP responses}

The next area of study was to verify within what muscle contraction range VEMPid and other forms of normalization could reliably be used. Different institutions use varying methods of controlling for muscle contraction. Most institutions request a certain minimal contraction level in order to obtain the cVEMP, because the cVEMP is an inhibitory response requiring at least some muscle contraction to inhibit. Requiring a minimum contraction level however does not eliminate the muscle contraction effect. In fact, the cVEMP amplitude grows almost linearly with muscle contraction effort, indicating that only a narrow range of muscle contraction should be allowed and this range should be similar from ear to ear and patient to patient. This has not been applied in clinic and is highly impractical. Not every patient is able to contract their neck muscles above a certain level, let alone trying to keep their contraction constant in a narrow range. Therefore, a variety of normalization methods have been proposed as an alternative and have shown to significantly decrease the variability caused by muscle contraction. These methods needed to be investigated to determine what the minimum required muscle contraction level and muscle contraction range should be in order to obtain reliable results. The minimum muscle contraction level that provides reliable results using the normalization techniques described in this thesis was lower than what had previously been proposed. Only a few studies systematically investigated the muscle contraction range within which normalization provides stable results and most studies only tested relatively high muscle contraction levels. Therefore, these studies generally recommended muscle contractions higher than the lower limit proposed in chapter 2.2. The use of a lower muscle contraction limit makes it easier for patients to obtain and maintain minimum muscle contraction. The range within which the normalization techniques provided stable results was identified, although the upper limit of this range was not explored in detail because 1) high muscle contractions were unnecessary, 2) asking patients to achieve high contraction levels is uncomfortable and 3) there are reports that found saturation of the CVEMP amplitude at high contraction levels, which would decrease reliability of normalization methods (Chapter 2.2). After determining how to reliably use cVEMP normalization methods, they could be tested in patient populations.

\section{The diagnostic value of cVEMP metrics and frequencies in SCD}

In order to use the previously described cVEMP metrics in clinic, their ability to differentiate patients from healthy controls needed to be evaluated. The diagnostic 
value of both the commonly used CVEMP threshold and the newer normalization metrics were compared to determine which metric or metrics were best at differentiating patients from controls. Besides using different cVEMP metrics, a variety of frequencies were used to obtain these cVEMP metrics. To determine which frequency best distinguished patients from controls, frequencies were compared. Finally, since the cVEMP appears to provide information regarding (patho)physiologic changes in the inner ear, it had been hypothesized that the cVEMP could provide information regarding symptomatology; a hypothesis that was tested in this thesis.

First, the CVEMP was evaluated in a group of patients with semicircular canal dehiscence syndrome (SCD), because previous reports indicated that the clinical utility of cVEMP is most promising in this patient group. To get an idea of how valuable the commonly used cVEMP threshold was in differentiating SCD from healthy controls, available cVEMP threshold data from a large group of SCD patients (239 ears) was analyzed. cVEMP thresholds alone provided sensitivities and specificities suboptimal for clinical use as a diagnostic tool. Because SCD patients often show low frequency audiometric air-bone gaps (ABG), this data was combined with the cVEMP thresholds, creating a "Third Window Indicator". This metric provided a more sensitive and specific means of detecting SCD (Chapter 3.1).

After determining that the CVEMP and $A B G$ provide important data regarding physiologic changes in the inner ear of SCD patients, it was investigated whether the CVEMP and ABG also provide information regarding symptomatology. Specifically, since SCD patients suffer from both auditory and vestibular symptoms, it was investigated whether these subjective symptoms corresponded with the objective ABG and CVEMP measures. As expected, a significant relationship between the subjective presence of hearing loss and ABG was found. However, the ABG and cVEMP did not significantly correlate with any other auditory or vestibular symptom, nor with the severity of symptoms. It is possible that the approach by which information on symptomatology was obtained was not uniform enough. Furthermore, the questionnaires used to quantify severity of symptoms may not have been precise enough to adequately capture details about SCD specific symptoms. Clinically, such a SCD specific questionnaire might aid in diagnosing SCD, would particularly be useful for accurate assessment of treatment outcomes and needs to be developed (Chapter 3.2).

Besides the commonly used cVEMP threshold, the diagnostic value of other (previously described) CVEMP metrics needed to be tested in the SCD population. The use of different normalization techniques to obtain the cVEMP is not common clinical practice. Therefore, prospective patient inclusion was warranted. Identifying the sensitivities and specificities to detect SCD in a large group of patients, using methods that have been used in clinic for years, provided a good starting point for evaluation of the diagnostic value of other (newer) cVEMP metrics in this patient population (Chapter 3.1). It allowed for comparison of the newer normalization methods to the status quo. The newer normalization methods (normalized peak-to-peak amplitude using trace-by- 
trace normalization and VEMP inhibition depth) were compared to the CVEMP threshold by testing a smaller group of SCD patients. The sensitivities and specificities obtained using the $500 \mathrm{~Hz}$ cVEMP threshold and third window indicator in this smaller patient group were similar to those found in the larger patient group described in Chapter 3.1. This indicates that this smaller patient group was representative of the larger group. The use of normalization techniques at $500 \mathrm{~Hz}$ somewhat improved the sensitivity compared to $500 \mathrm{~Hz}$ threshold. The clinical advantage of using the normalization techniques over the threshold is that it only requires one measurement on each side, decreasing testing time. A shorter, and preferably less costly, test potentially improves the accessibility of the test. Hopefully, this will increase the number of patients that can be screened, which may lead to a decrease in underdiagnosis of SCD patients. Furthermore, decreased testing time would decrease the discomfort of the test, which is specifically valuable in patients suffering from vestibular symptoms. In addition, the threshold is a subjective measure, increasing the risk for variability, while the normalization methods are objective metrics (Chapter 3.3). Besides using different cVEMP metrics, a variety of frequencies were used to obtain these metrics to investigate which frequency was best at differentiating SCD patients. The highest sensitivity and specificity was obtained with a $2000 \mathrm{~Hz}$ stimulus. This frequency provided the best sensitivity and specificity for all metrics with the normalized peak-to-peak amplitude and VEMPid, both reaching $96 \%$ sensitivity and $100 \%$ specificity. This is a clinically significant improvement when compared to the sensitivity (68\%; in combination with a 100\% specificity) obtained with a $500 \mathrm{~Hz}$ stimulus, which is the most commonly used frequency to obtain a cVEMP (Chapter 3.3).

\section{The diagnostic value of cVEMP in Menière's disease}

As previously stated, the cVEMP evaluates saccular function and because the saccule is involved in the disease process of Menière's disease (MD), the use of cVEMP in this patient population might be of interest. In the past, many different cVEMP metrics have been used to assess MD patients. However, differences in methodology between studies prevents the comparison of these metrics in the MD population and it has been unclear which metrics best detect pathology in these patients, if any. Therefore, a group of unilateral MD patients was prospectively tested using five different CVEMP metrics that have been used to study this population in the past. Similar to the SCD group, MD patients could best be differentiated from healthy controls using the threshold, normalized peak-to-peak amplitude and VEMPid. This may indicate that these metrics do a comparably good job of eliminating variability and can therefore most reliably detect saccular dysfunction. Unlike in the SCD group, the CVEMP does not achieve sensitivities and specificities (sensitivities of $80 \%$ in combination with specificities between 70 and $76.1 \%$ ) high enough for it to be used as a diagnostic tool for MD. There may be several reasons for this finding. Unlike in SCD, it is unclear what 
the exact pathophysiology of MD is, although many studies found a presence of saccular endolymphatic hydrops in these patients. It is unclear how this affects saccular function, but the fluctuating nature of the disease has led to the hypothesis that saccular function may be fluctuating in these patients as well. Therefore, the suboptimal sensitivities and specificities obtained with the CVEMP in MD may be due to the fluctuating nature of $\mathrm{MD}$, which may not always allow for the "saccular dysfunction" to be captured during cVEMP testing. In addition, the cVEMP may accurately test saccular (dys)function, but MD most likely entails more than fluctuating saccular function. For example, a temporal bone study, investigating the endolymphatic sac (thought to play a role in homeostasis of endolymphatic fluid) of patients with endolymphatic hydrops and a history of Menière's disease revealed two distinct pathologies: hypoplasia and degeneration of the endolymphatic sac. Patients with a hypoplastic versus a degenerated endolymphatic sac had distinct clinical characteristics. For example, hypoplastic endolymphatic sac pathology was associated with symptoms at a younger age, more severe symptomatology and the presence of bilateral disease compared to the patients with degeneration of their endolymphatic sac (1). This indicates that the pathology in MD is complex and that it is unclear how the underlying pathology (or pathologies) affect(s) saccular function. Irrespective of the underlying pathological mechanism in MD, it has now been established which CVEMP metrics best detect differences between MD patients and healthy subjects. These metrics may be used for follow-up of patients over time and to evaluate the effect of interventions such as intratympanic gentamycin injections. For example, the objective of intratympanic gentamycin injections in MD patients is to ablate the otolith organ hair cells and thereby to abolish remaining fluctuating function of these organs, reducing vestibular symptoms. If patients still suffer from vestibular symptoms after intratympanic gentamicin injection, it is possible that the injection was unsuccessful in eliminating otolith organ function and that the remaining function causes symptoms. The cVEMP could be used to determine the success of the gentamicin treatment by assessing the remaining saccular function and the potential need for another injection (Chapter 4.1).

\section{The predictive value of cVEMP in Menière's disease}

About a quarter to a third of patients with unilateral MD develop bilateral disease and it has been hypothesized that the patients with Menière-like cVEMPs in their "asymptomatic ear" would be the ones that become bilaterally affected in the future. An interesting observation noted by others in the past, is the difference in CVEMP outcomes between affected and unaffected MD ears. Some of the "asymptomatic ears" of patients with unilateral MD have Menière-like cVEMPs. This causes the average cVEMP thresholds of these patients to lie between the "MD affected" and "healthy control" ears. To investigate if patients with Menière-like cVEMPs in their 
asymptomatic ear are the ones developing bilateral disease, patients who have previously been diagnosed with unilateral MD and who underwent CVEMP testing at the time were contacted to determine who developed bilateral disease. The predictive value of the cVEMP in the "asymptomatic ear" was then evaluated. Previously established criteria for what defines a Menière-like and normal-like cVEMP revealed that patients with Menière-like cVEMPs have a $58.3 \%$ chance of developing bilateral disease, while patients with a normal-like cVEMP only have a $13.5 \%$ chance of developing disease. Although an attempt was made to limit the risk of bias by using previously established criteria (created by authors who did not know which patients would develop disease), these criteria were based on an educated guess. The positive and negative predictive values of the cVEMP were further improved by post hoc analyses of the data and establishing new revised criteria. A combination of the best three CVEMP metrics provided a positive and negative predictive value of $85.7 \%$ and 93.7\% respectively. Although the revised criteria were established retrospectively, these criteria were based on raw data without subjective influences or "educated guesses". The improved predictive values might allow physicians to more accurately inform patients about their chances of developing bilateral MD in the future (Chapter 4.2).

Considering the findings in this thesis, the CVEMP seems to be a promising diagnostic tool in SCD patients and a promising predictive tool in MD patients. However, further investigation of the use of the CVEMP in these patient populations is warranted. These future directions will be discussed below.

\section{Future directions}

\section{Using VEMPid to determine when to stop recording}

As laid out in this thesis, it has been unclear which cVEMP metrics and frequencies are best used in clinic. Therefore, some clinics will gather data using multiple cVEMP metrics at multiple frequencies. Especially obtaining threshold in both ears at multiple frequencies can be a time consuming endeavor. Furthermore, in a clinical setting, it is the technician who subjectively determines when enough data has been collected, which allows for variability. Besides the advantages of VEMPid over normalized peakto-peak amplitude and cVEMP threshold as described earlier, one potential application of the VEMPid would be to determine when to stop data collection. The accumulating VEMPid shown during data acquisition can aid in determining when the response stabilizes. However, visually determining when the response stabilizes is still a subjective appreciation and an objective criterion of a "stabilized response" has not been determined yet. In the future, subjects will need to be tested with a variety of data collection durations to analyze the stabilization of the response and to create an 
objective criterion that would allow for a more uniform use across audiologists and patients.

\section{Muscle contraction: How low can you go?}

Although the large majority of patients should be able to achieve the currently established "lower muscle contraction limit" without too much effort, older patients and patients with neck problems may benefit from an even lower limit. Determining the muscle contraction range within which CVEMP normalization techniques can reliably be used revealed that requesting patients to obtain very high muscle contraction levels was unnecessary. The upper limit of muscle contraction level was therefore not further explored. The lower limit however, should be explored further. The lower the muscle contraction level requested from a patient, the easier the test will be for the patient. It is currently unknown what the "real" lower limit is and further studies requesting systematic muscle contraction levels from the study subjects (as was done in Chapter 2.2) at low muscle contraction levels are necessary to determine this limit.

\section{Validating CVEMP metrics and frequencies in SCD patients}

Although the use of the different cVEMP metrics discussed in this thesis seem valuable as a diagnostic for SCD, these methods need to be validated in a larger group of subjects to assess reproducibility. The $500 \mathrm{~Hz}$ cVEMP threshold has been widely used in clinic and therefore, most data was available for this frequency and metric. This allowed for development of the $500 \mathrm{~Hz}$ Third Window Indicator in a large group of patients. As previously indicated, there is no worldwide consensus as to which CVEMP metrics should be used to analyze cVEMPs and methodology for obtaining CVEMP differs between institutions. Therefore, testing the use of the $500 \mathrm{~Hz}$ Third Window Indicator to verify its robustness against this methodological variability, using a multiinstitutional approach, would be appropriate. Since most clinics obtain $500 \mathrm{~Hz}$ thresholds this should be feasible. However, our studies also indicate that the use of a higher frequency, specifically the $2000 \mathrm{~Hz}$ tone burst, is superior regardless of which metric was used. Although this frequency showed superiority over the other tested frequencies in the same group of patients, less data was available to compare these frequencies and therefore, more data should be collected at this frequency to assess reproducibility. The same applies to the use of the newer normalization metrics. In addition, the use of these metrics and frequencies should be validated in a "real" patient population. This thesis revealed which metrics best detect pathology as compared to age-matched healthy controls, but did not assess the differentiation between patients with similar symptomatology as the patient population of interest. Currently, all metrics described in this thesis are used when clinically testing patients at the Massachusetts Eye and Ear. In addition, all patients suspected of having SCD based 
on symptomatology, audiograms and/or CT scans are tested with a $2000 \mathrm{~Hz}$ stimulus in addition to the standard clinical protocol.

\section{Optimizing SCD questionnaires}

SCD patients present with a host of different auditory and vestibular symptoms that can overlap with other otologic pathology. However, certain symptoms, such as the Tullio phenomenon are more specific for SCD. Therefore, the most commonly used hearing and dizziness questionnaires may not be specific enough for the evaluation of SCD. It is possible that, as presented in this thesis, subjective symptoms of SCD patients truly do not correlate significantly with objective measures such as audiograms and CVEMPs, but this conclusion cannot be drawn until more precise measures are evaluated first. Both the Massachusetts Eye and Ear and Maastricht University Medical Center are working on developing more specific SCD targeted questionnaires.

\section{Validating CVEMP metrics in MD patients}

Determining which cVEMP metrics best detect pathology in MD patients was an important step. Specifically because a wide range of metrics have been used in this patient population and the use of different metrics across institutions is far from uniform. Similar to the future steps in the SCD population, the next step for MD patients is to validate the use of different metrics in a population with Menière-like symptomatology as opposed to a group of age-matched healthy controls. Another step would be to systematically test MD patients over time to assess CVEMP changes over time. The cVEMP could also be used to compare pre- and post-treatment status, both for current and new therapies.

\section{Validating CVEMP predictive value in MD patients}

Both previously developed criteria for Menière-like CVEMP as well as the new revised criteria discussed in Chapter 4.2 are promising predictive tools for who develops bilateral MD. This would allow for more accurate patient counseling. The retrospective single-institution study performed to obtain this information is a limitation and a large prospective multi-institutional study would be ideal to verify the predictive abilities of the CVEMP. This type of study would be very time consuming given that it can take decades before unilateral MD patients develop bilateral disease. To get additional information on this matter in a more timely manner, a retrospective version of a large multi-institutional study would be appropriate.

This thesis illustrates that the CVEMP is a promising diagnostic tool in SCD patients and a promising predictive tool in MD patients. However, certain aspects require further investigation to optimize its usefulness in a clinical setting. 


\section{Reference}

1. Eckhard AH, Zhu M, O'Malley JT, et al., Inner ear pathologies impair sodium-regulated ion transport in Meniere's disease. Acta Neuropathol 2019;137(2):343-57. 



\section{Chapter 6}

Summary 



\section{Summary}

Dizziness and balance problems can have many different etiologies. One important contributor to balance is the vestibular organ, located in the inner ear. Each vestibular organ consists of five components. For patients with balance problems caused by vestibular pathology it would be ideal to assess each of the five vestibular components separately to identify exactly which part (or parts) of the vestibular organ is the source of the problem. This information could potentially 1) aid in diagnosis, 2) predict development of disease, 3 ) be used to find the source of the problem and therefore target of treatment, and 4) be useful to evaluate the effect of treatment and potential preventive measures, this last being a particularly important possibility as we see a rapid increase in clinical trials of new inner ear drugs. To date, the vestibular evoked myogenic potential is the only test able to evaluate the saccule and the utricle separately. Besides calorics, which can be used to assess each horizontal canal, other vestibular tests can either evaluate a combination of the five vestibular sense organs and/or cannot distinguish the left from the right ear. This thesis focused on the cervical vestibular evoked myogenic potential, which can evaluate the function of each saccule (left and right) separately.

First, a specific approach developed to remove variability from factors other than saccular function, the VEMP inhibition depth (VEMPid), was tested in a group of healthy subjects. Removing this variability is essential for accurate comparisons of saccular function in different patient groups. The calculation of VEMPid in a patient population requires using a generic template generated from responses of healthy subjects, instead of the originally used subject-specific template. The VEMPids calculated with generic versus subject-specific templates in a group of healthy volunteers were very similar (correlation coefficient $=0.989$ ). No significant differences in calculating VEMPid with the subject-specific versus generic template were found across frequencies and sound levels ( $p$ values between 0.798 and 0.886 ). In conclusion, a generic template can reliably be used to obtain the VEMP inhibition depth. This was a first step toward its use in patients with vestibular pathology (Chapter 2.1).

Next, it has been widely known that muscle contraction level affects the cVEMP amplitude and that this can be compensated for by normalization, but it was unclear within what muscle contraction range different normalization techniques, such as VEMPid, can reliably be used. In a group of healthy subjects of varying ages, it was confirmed that muscle contraction strongly affects the CVEMP amplitude $(p<0.001)$ and that the normalization methods described in this thesis provided stable results. Within a muscle contraction range of $45-300 \mu \mathrm{V}$ root mean square (rms), there was no significant effect of muscle contraction on the normalized peak-to-peak amplitude $(p=0.713)$ and VEMP inhibition depth $(p=0.546)$. This indicates that the use of these 
normalization techniques does not require strong neck muscle contractions. Using weak-to-moderate muscle contractions decreases patient discomfort and allows patients, especially older patients, to complete the test (Chapter 2.2).

After optimizing these normalization techniques, they were used in patient populations. To assess how well the cVEMP threshold can distinguish healthy controls from semicircular canal dehiscence syndrome (SCD) patients, data were retrospectively obtained from a large group of SCD patients. Using solely CVEMP thresholds, sensitivities and specificities for diagnostic use were calculated. However, combining cVEMP threshold with the $250 \mathrm{~Hz}$ air-bone gap increased the sensitivity and specificity to $82 \%$ and $100 \%$ respectively, making the use of this combined data, referred to as the "Third Window Indicator" a promising and easy to use tool for detecting SCD (Chapter 3.1).

To assess whether CVEMP outcomes were associated with the presence and severity of symptoms, the relationship of SCD patient symptoms with their objective audiometric and CVEMP test results was evaluated. It was hypothesized that patients with lower cVEMP thresholds and larger audiometric air-bone gaps would be more symptomatic. As expected, hearing loss was associated with larger audiometric air-bone gaps (ABG). However, none of the other auditory or vestibular symptoms correlated significantly with $A B G s$ or cVEMP thresholds. Whether this was due to a true absence of a correlation between these subjective and objective measures, or if a more SCD-specific questionnaire would reveal a relationship, remains to be explored. A strong relationship between outcomes of subjective (questionnaires) and objective (cVEMPs) tools could aid in diagnosis and treatment-effect assessment (Chapter 3.2).

To compare how well the different CVEMP metrics (the previously used threshold and the newer normalization techniques) at four different frequencies could differentiate dehiscent from healthy ears, data were collected prospectively. The optimal combination of sensitivity and specificity was found using a $2000 \mathrm{~Hz}$ sound stimulus, which is a higher frequency than the most commonly used $500 \mathrm{~Hz}$ stimulus. The $2000 \mathrm{~Hz}$ threshold and the TWI metric both reached a sensitivity of 92\%, while the $2000 \mathrm{~Hz}$ normalized peak-to-peak amplitude (VEMPn) and the VEMPid both reached a sensitivity of $96 \%$ in combination with $100 \%$ specificity. The sensitivities and specificities reached with the $2000 \mathrm{~Hz}$ stimulus make the CVEMP an even more promising diagnostic tool for SCD. Furthermore, using the VEMPn or VEMPid at only one frequency will greatly reduce testing time, potentially making the CVEMP a more accessible test and aiding in the decrease of SCD miss- and under-diagnosis (Chapter 3.3).

As in SCD patients, it was known that cVEMP outcomes (thresholds, amplitudes, interaural asymmetry ratios and tuning ratios) in Menière's disease (MD) patients differ 
significantly from healthy controls. However, it was unclear which CVEMP metric and frequency were best at differentiating MD patients from healthy controls and how accurate they were in doing so. A group of MD patients and age matched controls were prospectively included and tested at different frequencies. The $500 \mathrm{~Hz}$ cVEMP threshold, VEMPn and VEMPid were similarly good at differentiating MD from healthy ears with optimal sensitivities and specificities of at least $70 \%$ and $80 \%$. These percentages indicate that the CVEMP may not be a great diagnostic tool for MD, but has potential to serve as a follow-up tool and to evaluate the effect of interventions (Chapter 4.1).

Finally, patients with MD have about a $25-35 \%$ risk of developing bilateral disease. It was hypothesized that the asymptomatic ears of unilateral MD patients with "Menièrelike" cVEMPs were most likely to develop bilateral disease. To test this hypothesis, old cVEMP data from a patient group previously diagnosed with unilateral MD were analyzed to assess whether their cVEMP was predictive for development of bilateral disease. Using previously established criteria for "Menière-like" cVEMP outcomes, it was concluded that the "Menière-like" ears were more likely (58.3\%) to develop bilateral disease compared to the "normal-like ears" (13.5\%). Post hoc data analysis, using a combination of the most promising CVEMP metrics, improved the positive predictive value to $85.7 \%$ and the negative predictive value to $93.7 \%$, indicating that these "revised criteria" were superior to the originally suggested criteria for predicting which patients develop bilateral disease. The predictive ability of cVEMP might improve counseling of MD patients regarding their risk of developing bilateral disease in the future (Chapter 4.2).

This thesis demonstrates that the CVEMP has great clinical potential to function as a diagnostic test in SCD patients as well as a means to follow MD patients and to predict future bilateral disease. To further assess and establish the clinical value of cVEMP, the cVEMP measures studied in this thesis need to be prospectively validated in larger patient groups with a multitude of vestibular pathologies. 



\section{Chapter 7}

Dutch summary - Nederlandse samenvatting 



\section{Nederlandse samenvatting}

Duizeligheid en evenwichtsproblemen kunnen vele verschillende oorzaken hebben. Het evenwichtsorgaan (vestibulair orgaan) in het binnenoor levert een belangrijke bijdrage aan evenwicht. Elk vestibulair orgaan bevat vijf onderdelen. Voor patiënten met evenwichtsproblemen die veroorzaakt worden door vestibulaire pathologie zou het ideaal zijn om elke van deze vijf vestibulaire onderdelen apart te kunnen evalueren om precies te identificeren welk deel (of delen) van het vestibulaire orgaan de oorzaak van het probleem is. Deze informatie zou gebruikt kunnen worden voor 1) diagnosticering, 2) het voorspellen van ziekte 3) het identificeren van de oorzaak van het probleem en daarmee het doelwit voor behandeling, en 4) evaluatie van het behandeleffect en potentieel preventieve maatregelen. Dit laatste is met name belangrijk gezien de toename van het aantal clinical trials voor medicatie voor het binnenoor. De vestibular evoked myogenic potential is tot op heden de enige test die de functie van de sacculus en utriculus (twee van de vijf sensoren in het vestibulaire apparaat) apart kan evalueren. Met calorisch onderzoek kan met name elk horizontale halfcirkelvormig kanaal afzonderlijk getest kan worden. Alle andere vestibulaire testen kunnen alleen een combinatie van de vijf vestibulaire sensoren evalueren en/of kunnen geen onderscheid maken tussen het linker en het rechteroor. Dit proefschrift richt zich op de cervical vestibular evoked myogenic potential (cVEMP), waarmee de functie van elke sacculus (links en rechts) apart geëvalueerd kan worden.

Allereerst is een specifieke benadering, de VEMP inhibition depth (VEMPid), ontwikkeld om de variabiliteit van factoren anders dan de functie van de sacculus te elimineren, getest in een groep gezonde proefpersonen. Het elimineren van deze variabiliteit is essentieel om de functie van de sacculus in verschillende patiëntengroepen betrouwbaar te kunnen vergelijken. Om de VEMPid te kunnen berekenen in een patiëntenpopulatie is een generieke template (voorbeeld van hoe een cVEMP eruit zou moeten zien) nodig, gegenereerd van gezonde proefpersonen, in plaats van de originele subject-specifieke template. De VEMPid's berekend met een generieke versus een subject-specifieke template in een groep gezonde proefpersonen waren zeer vergelijkbaar (correlatie coëfficiënt $=0.989$ ). Er waren geen significante verschillen tussen VEMPid's berekend met de subject-specifieke versus de generieke template over verschillende frequenties en geluidsterktes ( $p$ waardes tussen 0.798 en 0.886). In conclusie, een generieke template kan betrouwbaar gebruikt worden om de VEMP inhibition depth te verkrijgen. Dit was een eerste stap richting het gebruik van deze methode in patiënten met vestibulaire pathologie (Hoofdstuk 2.1).

Het is bekend dat de mate van spierspanning de amplitude van de cVEMP respons beïnvloedt en dat hiervoor gecorrigeerd kan worden met normalisatie technieken. Het was echter niet bekend binnen welke spierspanningsrange verschillende normalisatie 
technieken, zoals VEMPid, betrouwbaar gebruikt zou kunnen worden. In een groep gezonde proefpersonen van variërende leeftijden werd bevestigd dat spierspanning de amplitude van de cVEMP sterk beïnvloedt $(p<0.001)$ en dat de normalisatiemethodes beschreven in dit proefschrift stabiele resultaten geven. Er was geen significant effect van spierspanning op de genormaliseerde amplitude $(p=0.713)$ en VEMP inhibition depth $(p=0.546)$ bij een spierspanning tussen $45-300 \mu \mathrm{V}$ root mean square. Dit geeft aan dat het gebruik van deze normalisatietechnieken geen sterke spierspanning vereist. Het gebruik van zwakke tot matige spierspanning vermindert het ongemak voor de patiënt en stelt hen, met name oudere patiënten, in staat om de test te voltooien (Hoofdstuk 2.2).

Nadat deze normalisatietechnieken geoptimaliseerd waren, konden zij gebruikt worden in een patiëntenpopulatie. Om vast te stellen hoe goed de cVEMP drempel gezonde proefpersonen kan onderscheiden van patiënten met superieure halfcirkelvormige kanaal dehiscentie syndroom (SCD), werd retrospectief data verzameld van een grote groep SCD patiënten. De sensitiviteit en specificiteit werden berekend, uitsluitend gebruik makend van de cVEMP drempel. Het combineren van de CVEMP drempel met $250 \mathrm{~Hz}$ air-bone gap data verhoogde de sensitiviteit en specificiteit naar respectievelijk $82 \%$ en $100 \%$, wat het gebruik van deze combinatie, aangeduid als "Third Window Indicator" een veelbelovende en makkelijk te gebruiken methode voor detectie van SCD maakt (Hoofdstuk 3.1).

De relatie tussen SCD-symptomen en objectieve audiometrische en cVEMP test resultaten werden geëvalueerd om te onderzoeken of cVEMP uitkomsten geassocieerd waren met de aanwezigheid en mate van symptomen. De hypothese was dat patiënten met lage cVEMP thresholds en grote air-bone gaps (ABG) meer symptomatisch zouden zijn. Zoals verwacht was gehoorverlies geassocieerd met grote ABGs. Geen van de andere auditieve of vestibulaire symptomen was echter significant gecorreleerd aan ABGs of cVEMP thresholds. Of dit komt door een ware afwezigheid van een correlatie tussen deze subjectieve en objectieve maten, of dat een meer SCD-specifieke vragenlijst wel een relatie zou onthullen, dient verder onderzocht te worden. Een sterke relatie tussen subjectieve (vragenlijsten) en objectieve (cVEMP) middelen zou het makkelijker maken om deze aandoening te diagnosticeren en zou nuttig kunnen zijn voor evaluatie van een eventueel behandeleffect (Hoofdstuk 3.2).

Om te vergelijken hoe goed verschillende cVEMP uitkomstmaten (de eerder gebruikte drempel en nieuwere normalisatietechnieken) SCD van gezonde oren kunnen onderscheiden, werd prospectief data verzameld gebruik makend van vier verschillende frequenties. De optimale combinatie van sensitiviteit en specificiteit werd gevonden bij gebruik van een $2000 \mathrm{~Hz}$ geluidsstimulus, wat een hogere frequentie is dan de meest gebruikte $500 \mathrm{~Hz}$ stimulus. De $2000 \mathrm{~Hz}$ drempel en "Third Window 
Indicator" bereikte beide een sensitiviteit van 92\%, terwijl de $2000 \mathrm{~Hz}$ genormaliseerde peak-to-peak amplitude (VEMPn) en de VEMPid beide een sensitiviteit van $96 \%$ bereikten, in combinatie met een specificiteit van 100\%. De sensitiviteit en specificiteit die bereikt konden worden met de $2000 \mathrm{~Hz}$ stimulus maken de cVEMP een veelbelovende diagnostische test voor SCD. Daarnaast zou het gebruik van de VEMPn en VEMPid op 1 frequentie de testtijd behoorlijke reduceren, waardoor de CVEMP waarschijnlijk toegankelijker wordt, wat vervolgens bij kan dragen aan een afname van mis- en onderdiagnose van SCD (Hoofdstuk 3.3).

Net als in patiënten met SCD was het bekend dat CVEMP uitkomstmaten (drempels, amplitudes, inter-aural asymmetry ratio's en tuning ratio's) in de ziekte van Menière (MD) significant verschillen van gezonde controles. Het was echter niet duidelijk welke cVEMP uitkomstmaten en frequentie het best waren om MD-patiënten van gezonde controles te onderscheiden en hoe nauwkeurig deze maten zijn. Een groep MD-patiënten en gezonde controles (gematcht voor leeftijd) werden daarom prospectief geïncludeerd en getest op verschillende frequenties. De $500 \mathrm{~Hz}$ cVEMP drempel, VEMPn en VEMPid waren even goed in het differentiëren van MD en gezonde oren met een optimale sensitiviteit en specificiteit van ten minste 70 en $80 \%$. Deze suboptimale percentages geven aan dat de cVEMP wellicht geen goed diagnosticum is voor MD, maar dat er potentieel is om te fungeren als follow-up test en om het effect van interventies te evalueren (Hoofdstuk 4.1).

Tot slot, patiënten met MD hebben een kans van ongeveer $25-35 \%$ op het ontwikkelen van bilaterale ziekte. Er is gesuggereerd dat de asymptomatische oren van unilaterale MD-patiënten met "Menière-typerende" cVEMPs een grotere kans hebben om bilaterale ziekte te ontwikkelen. Om deze hypothese te testen werd oude data van een groep patiënten die eerder gediagnosticeerd waren met unilaterale MD, geanalyseerd om te onderzoeken of de cVEMP voorspellend was voor het ontwikkelen van bilaterale ziekte. Gebruik makend van eerder ontwikkelde criteria voor "Menière-typerende" cVEMP uitkomsten kon geconcludeerd worden dat deze oren een grotere kans hadden (58.3\%) om ziekte te ontwikkelen in vergelijking met de "normaal-typerende" oren (13.5\%). Post hoc data-analyse, gebruik makend van een combinatie van de meest veelbelovende cVEMP maten, verbeterde de positief voorspellende waarde naar $85.7 \%$ en de negatief voorspellende waarde naar $93.7 \%$, wat aangeeft dat deze "gereviseerde criteria" superieur waren ten opzichte van de originele criteria om te voorspellen welke patiënten bilaterale ziekte ontwikkelen. Het voorspellend vermogen van de cVEMP zou de counseling van MD-patiënten met betrekking tot hun risico op het ontwikkelen van bilaterale ziekte in de toekomst kunnen verbeteren (Hoofdstuk 4.2).

Dit proefschrift toont aan dat de cVEMP potentie heeft om als diagnostische test te fungeren voor SCD, voor follow-up van MD-patiënten en om het ontwikkelen van 
bilaterale ziekte in de toekomst te voorspellen. Om de klinische waarde van de CVEMP verder te onderzoeken moeten de cVEMP uitkomstmaten die in dit proefschrift bestudeerd zijn, prospectief gevalideerd worden in grotere patiënten groepen met een verscheidenheid aan vestibulaire pathologie. 


Acknowledgements - Dankwoord 



\section{Acknowledgements - Dankwoord}

Most importantly, I would like to thank all study participants. I am truly grateful for your enthusiasm and time without which this thesis never would have been completed. As many of you have stated: hopefully, the results of this thesis will improve clinical practice and be beneficial to patients in the future.

Steven Rauch, if it was not for your passion for this research, support, patience, and trust in me I do not know what would have become of this PhD (and me!). You have given me the opportunity, space and time to grow as a researcher and as a person. Besides spending time in the lab, I also had the privilege to spend time with you in clinic and have great admiration for your ability to explain complicated matter in a way that anyone (really anyone!) can understand. Besides a great teacher, scientist, and clinician, you are also one of the best human beings I know. Your moral compass is pointing in the right direction, which has made it an absolute pleasure to work with you. I will miss your great analogies ("figures are like baby pictures...") and might borrow one or two in the future. You really are the best mentor I could have wished for. I know that you are not a fan of hyperbolical expressions of praise, but I assure you that I meant every word. Thank you!

Herman Kingma, ik wil je ontzettend bedanken voor de mogelijkheid mijn promotieonderzoek bij jou te voltooien. Ondanks je drukke schema (en de soms gekke tijdstippen van onze telefoontjes door het tijdsverschil) stond jij mij altijd te woord met een glimlach. Het feit dat je, zelfs na je pensioen, nog zo betrokken bent geeft wel aan hoeveel passie je hebt voor het vak. Ontzettend bedankt!

Raymond van de Berg, zonder jouw betrokkenheid, positiviteit en enthousiasme was dit proefschrift nooit tot een voltooiing gebracht. De snelheid waarmee jij mijn e-mails beantwoord is ongekend. Ik weet niet hoe je het doet, maar je weet altijd ontzettend nuttige en precieze aanwijzingen te geven zonder dat het als kritiek klinkt. Nooit eerder was ik klaar met het aanpassen van een rood gemarkeerd manuscript en voelde ik me achteraf zo goed. Ik wil je ontzettend bedanken en hoop in de toekomst nog veel van je te leren.

John Guinan, as the engineer on the "VEMP team" your contribution to this work has been extremely important. In fact, this thesis never would have been completed without your help. The patience with which you answered all my questions is admirable and I truly believe you taught me how to think like a scientist. Thank you for your dedication to this work. It has been a pleasure to work with you. 
Barbara Herrmann, you have been there to guide me through this PhD from the moment I started and I cannot thank you enough for all those hours you spent teaching me how to perform a study from writing the proposal to publication of the work. Your ability to tie research and clinic together are truly aspirational. Thank you for everything!

Daniel Lee, I am very grateful for the opportunity you gave me to come work with you in Boston. If you had not taken me under your wings as a medical student, I never would have even started this PhD. Your excitement and devotion to your work in clinic and in the lab have been a great inspiration for me. It is amazing to see how you manage such a diverse research interest and excel at them too! Even after I left your lab, you were always very interested, engaging and supportive for which I am extremely thankful.

Mark van Tilburg, als jij mijn voorganger toch niet was... Er werd mij van de voren verteld dat ik hele grote schoenen te vullen had en ik kan alleen maar hopen dat ik in de buurt ben gekomen van de verwachtingen. Zonder jouw werk had ik dit onderzoek nooit kunnen doen. We hebben maar weinig tijd samen in Boston doorgebracht, maar het was toch altijd heel gezellig. Ontzettend bedankt voor al je hulp!

Piotr Marciniak, the patience with which you have helped me in L1 is truly admirable. From near and far (I think you were on a beach in Italy ones) you always managed to come to the rescue. The fact that you always walk in with a big smile makes collaborating with you even more fun. Thank you!

Dear Audiologists, all of your interest and engagement in our studies have made patient recruitment so much easier. It is always a pleasure to work with such a friendly and fun group of people.

Hugh Curtin, I still cannot believe the amount of time you personally spent going through CT scans with me. Somehow you always found time in your busy schedule to teach and I can say that there is really no better person to learn from. Thank you for your time, patience and support.

Heidi Nakajima, I cannot express with words how thankful I am that you have accepted me as your honorary lab member. All the lunches, ice-cream afternoons and weekend brunches with your lab have made my experience in Boston even better. Besides the social engagement, I am also very thankful for your interest in my work. Thank you for being my honorary mentor.

Elliott Kozin, I am not sure what would have happened if you did not guide me during my first steps as a medical student at Mass. Eye and Ear. Even though you are always 
working on 100 other papers (or more, I cannot keep track) you always take time to teach and mentor me when I need you. I feel very lucky that I got to work with you and I am excited to see what your next steps will be.

David Jung and Aaron Remenschneider, I would like to thank both of you for your selfless engagement and willingness to answer my research and clinic related questions. It has been a pleasure to work with you!

Barbara Fullerton and Charles Liberman, thanks to you I had the opportunity to engage in the SHBT program and learn the basics of speech, hearing and balance. You are both great teachers and I would like to thank you for your support and opportunity to learn from the best.

Barbara Beckman-Beard, Melissa McKinnon, Harsha Radhakrishnan and Andrea Zweibel, thank you for your help with navigating the administration that is involved with research. Your availability and willingness to help made my life a lot easier.

Chris and Margie Brown, the hospitability with which you and your family welcomed me to the Boston area is unprecedented. I have such great memories of all the thanksgivings, super bowl parties and hockey games (including flaming puck) and really consider you my American family. Thank you for everything!

Hans Borgstein, Liane Tan en Sara Hakim, jullie enthousiasme voor de KNO is een van de redenen waarom ik zo geïnteresseerd ben geraakt in het vak. Jullie aanmoediging om onderzoek te doen heeft zeker bijgedragen aan mijn motivatie om dit promotietraject in te gaan. De belangstelling die jullie daarna steeds hebben getoond was ontzettend bemoedigend en ik hoop dat we nog lang in contact blijven!

Bing Tan, tijdens mijn allerlaatste coschap in het AvL heb ik ontzettend veel van $u$ geleerd. Uw enthousiasme voor het vak heeft zeker aanstekelijk gewerkt en ik wil u ontzettend bedanken voor uw interesse en aanmoediging om dit carrière pad in te slaan.

Aly, my first "Bostonian" friend! How would I have gotten to know the city without you?! I have such great memories of our time both in and outside the lab and am so happy that we still visit each other. Thank you for being such a great friend.

Kevin and Maria, it was such a pleasure to work with both of you! Thank you so much for all the time you put in to make our SCD studies happen. 
Darcy, Kristine, Salwa, Song, and Stefan, my honorary lab! 2019 really was the year of the defense (or was it 2020?). Going through this PhD process with all of you has made it so much easier. Even though our research topics were variable, I feel like I have learned so much from each of you. I will miss you all tremendously.

Jan, Jenny and Jessica, I cannot find the words to express how valuable your friendship is to me. The three of you have really been there for me through the good and difficult times for which I am so grateful. Thank you for always being there!

Becky, Dave, Kirupa, Tyler, Vivek and Xiying, even though I was still a PhD student, you postdocs (and resident) made me feel like 'one of your own'. I have had so much fun with all of you (all the AROs)! You have all been very supportive on a professional level as well and I cannot wait to see what all of you will do next.

Nina, the bestest roommate, supermodel and cheerleader. I cannot believe the amount of encouragement, support and love you have given me. Thank you!

Aleid, Anneke, Atie, Christina, Door, Esther, Eveline, Irene, Josephine en Liesbeth (bloedzusters), ook al zie ik jullie niet vaak, als we elkaar zien is het altijd weer net zo gezellig als op de poli. Jullie zijn altijd zo enthousiast en geïnteresseerd en dat geeft mij ontzettend veel steun. Laten we die strandreünie erin houden.

Maura, mijn earbuddy! Ik heb hele goede herinneringen aan onze tijd samen in Boston. Toch heel fijn om af en toe even in het Nederlands te kunnen kletsen. Ik ben ontzettend trots op jou en hoop dat we nog lang earbuddies blijven.

Tessa en Rianne, volleybal en sushi friends! Ook al zijn jullie ver weg, zo voelt het eigenlijk niet. Ik ben blij dat we elkaar via de app zo goed op de hoogte houden. Het is heel fijn om te weten dat als ik jullie dan eindelijk zie tijdens onze sushimomentjes, alles weer net is als vanouds. Heel erg bedankt voor jullie interesse, enthousiasme en steun.

Charlotte, Ghislaine, Ivar, Nicky en Suus, vanaf de middelbare school (en eerste jaar geneeskunde) zijn jullie al mijn beste vriendjes en ben ontzettend blij dat jullie dat nog steeds zijn. Ook al mis ik jullie heel erg, jullie zijn altijd maar een appje of telefoontje bij me vandaan. Ik kijk er altijd erg naar uit om jullie weer te zien in december en voel mij heel gelukkig als ik iedereen weer bij elkaar heb gekregen tijdens mijn korte bezoekjes. Ontzettend bedankt voor alle steun en liefde!

Anouk, ik heb zoveel goede herinneringen aan alles wat we samen hebben meegemaakt. $\mathrm{Er}$ is niemand met wie ik liever de volledige middelbare school én de geneeskunde opleiding had willen doorlopen. Ook al ben ik ver weg en mis ik je 
ontzettend, jij bent er nog steeds altijd voor mij, in goede en in slechte tijden. Ik ben ontzettend blij je ook tijdens mijn verdediging naast mijn zijde te hebben als paranimf.

Hans, Lida, Marc en Tessa (v/d Brandtjes), de eersten die ik op kom zoeken als ik even in Nederland ben. Jullie zijn als familie voor mij en ook al mis ik jullie erg, ik weet dat jullie er altijd voor mij zijn. Een hele dikke knuffel!

Herman, Jacqueline, Ineke, Habib, Ruud, Lore en Margit (lieve ooms en tantes), ik wil jullie ontzettend bedanken voor de interesse die jullie altijd tonen. Ik zie jullie niet vaak, maar voel me altijd heel erg gesteund via de app. Een dikke kus voor jullie allemaal.

Lieve Oma's, het is niet altijd makkelijk om zover weg te zijn. Ik wil jullie ontzettend bedanken voor alle liefde en kijk uit naar de vele Skype sessies die we nog zullen hebben!

Lieve papa en mama, waar moet ik beginnen? Jullie hebben mij zo ontzettend gesteund in dit hele proces (zoals altijd). Als ik het even moeilijk had waren jullie er altijd voor mij. Jullie onvoorwaardelijke liefde is het grootste cadeau wat jullie mij kunnen geven. Ik mis jullie ontzettend, maar gelukkig zien we elkaar wekelijks tijdens onze Facetime sessies (soms inclusief hele voetbal of volleybalwedstrijden) en komen jullie mij een paar keer per jaar opzoeken. Zoals Daan het al eens zei, met zijn vieren zijn wij beste team dat ik mij kan voorstellen: team Noij!

Lieve Daan, mijn lievelingsbroer! Ik ben zo ontzettend blij dat wij samen zijn opgegroeid (en nog steeds samen groeien). Jij bent er altijd voor mij en ik denk niet dat je weet hoeveel ik eraan heb gehad dat jij het promotietraject al hebt doorlopen. Zoals altijd was je mijn persoonlijke adviseur waardoor ik precies wist wat mij te wachten stond. Ik ben ontzettend trots op jou en alles wat je al bereikt hebt. Ik had mij geen betere broer en paranimf kunnen wensen!

Dear Jordan, kochanie. Somehow, you are able to make me feel like the best version of myself. You always make me laugh and can make me feel better in any situation, which has helped tremendously going through this PhD process. You have been extremely supportive and I love how you even manage to listen to my practice talks - although you think I study vamps. I admire your selflessness and tireless efforts to help others (including me). You are the best partner I could ever wish for and I am looking forward to have you by my side for a long time. Kocham cię. 

Bibliography 



\section{Bibliography}

Noij KS, Herrmann BS, Guinan JJ Jr, Rauch SD. Predicting development of bilateral Meniere's disease based on cVEMP threshold and tuning. 2019 Accepted for publication in Otology \& Neurotology.

Chemtob RA, Noij KS, Qureshi AA, Klokker M, Nakajima HH, Lee DJ. Superior Canal Dehiscence Surgery Outcomes Following Failed Round Window Surgery. Otol Neurotol. 2019 Apr;40(4):535-542.

Noij KS, Herrmann BS, Guinan JJ Jr, Rauch SD. Cervical Vestibular Evoked Myogenic Potentials in Menière's Disease: A Comparison of Response Metrics. Otol Neurotol. 2019 Mar;40(3):e215-e224.

Noij KS, Herrmann BS, Guinan JJ Jr, Rauch SD. Toward Optimizing cVEMP: 2,000-Hz Tone Bursts Improve the Detection of Superior Canal Dehiscence. Audiol Neurootol. 2018;23(6):335-344

van Tilburg MJ, Herrmann BS, Rauch SD, Noij KS, Guinan JJ Jr. Normalizing cVEMPs: Which Method Is the Most Effective? Ear Hear. 2018.

Noij KS, Wong K, Duarte MJ, Masud S, Dewyer NA, Herrmann BS, Guinan JJ Jr, Kozin ED, Jung DH, Rauch SD, Lee DJ. Audiometric and CVEMP Thresholds Show Little Correlation With Symptoms in Superior Semicircular Canal Dehiscence Syndrome. Otol Neurotol. 2018 Oct;39(9):1153-1162.

Noij KS, van Tilburg MJ, Herrmann BS, Marciniak P, Rauch SD, Guinan JJ Jr. Toward Optimizing VEMP: Calculating VEMP Inhibition Depth With a Generic Template. Ear Hear. 2018;39(6):1199-1206.

Noij KS, Duarte MJ, Wong K, Cheng YS, Masud S, Herrmann BS, Curtin HD, Kanumuri VV, Guinan JJ Jr, Kozin ED, Tarabichi O, Jung DH, Lee DJ, Rauch SD. Toward Optimizing Cervical Vestibular Evoked Myogenic Potentials (cVEMP): Combining Air-Bone Gap and cVEMP Thresholds to Improve Diagnosis of Superior Canal Dehiscence. Otol Neurotol. 2018 Feb;39(2):212-220.

Noij KS, Herrmann BS, Rauch SD, Guinan JJ Jr. Toward Optimizing Vestibular Evoked Myogenic Potentials: Normalization Reduces the Need for Strong Neck Muscle Contraction. Audiol Neurootol. 2017;22(4-5):282-291 
Noij KS, Noij DP, Borgstein J. Healing of the tympanic membrane after surgical intervention for atelectasis of the middle ear in sixty-two adults. Clin Otolaryngol. 2017 Apr;42(2):450-454.

Noij KS, Kozin ED, Sethi R, Shah PV, Kaplan AB, Herrmann B, Remenschneider A, Lee DJ. Systematic Review of Nontumor Pediatric Auditory Brainstem Implant Outcomes. Otolaryngol Head Neck Surg. 2015 Nov;153(5):739-50.

Noij KS, Remenschneider AK, Kozin ED, Puram S, Herrmann B, Cohen M, Cunnane MB, Lee DJ. Direct parasagittal magnetic resonance imaging of the internal auditory canal to determine cochlear or auditory brainstem implant candidacy in children. Laryngoscope. 2015 Oct;125(10):2382-5. 




\section{Curriculum vitae}





\section{Curriculum vitae}

Kim Noij was born on December 20, 1989 in Badhoevedorp, the Netherlands. She grew up in Hoofddorp, the Netherlands, with her parents and her brother Daan. Kim obtained her Atheneum (high school) diploma from the Katholieke Scholengemeenschap Hoofddorp in 2008. From 2008 to 2015 she went to Medical School at the VU University Medical Center in Amsterdam. In between rotations, she worked as a phlebotomist with a group of enthusiastic and encouraging colleagues at the Spaarne hospital in Hoofddorp.

During her first year of rotations she discovered a love for

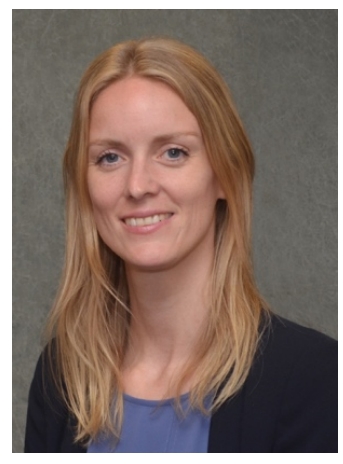
Otolaryngology - Head and Neck Surgery and decided to pursue a 16 week Otolaryngology sub-internship with Dr. Hans Borgstein at Tergooi hospital in Blaricum This internship confirmed her excitement for Otolaryngology and ignited an interest in research. Kim decided to explore her research interest and spent a 5 month research internship studying the auditory brainstem implant at the Massachusetts Eye and Ear and Harvard Medical School in Boston with Dr. Daniel Lee. During her time in Boston, she met Dr. Steven Rauch, director of the Balance and Vestibular center at the Massachusetts Eye and Ear and Professor of Otolaryngology at Harvard Medical School. He was studying cervical vestibular evoked myogenic potentials and invited Kim to come back to Boston to pursue a PhD in Hearing and Vestibular science, which would be in collaboration with Prof. Dr. Herman Kingma and Dr. Raymond van de Berg at Maastricht University Medical Center in the Netherlands. Before she was able to start her PhD, Kim went back to Amsterdam to finish Medical School with sub-internships in General Surgery and Emergency Medicine. Because of her interest in Head and Neck surgery, Kim did an additional sub-internship in Head and Neck Surgery at the Netherlands Cancer Institute - Antoni van Leeuwenhoek hospital with Prof. Dr. Bing Tan, after which she returned to Boston.

All chapters of this thesis have been written and published in international peerreviewed journals during her time at the Massachusetts Eye and Ear under supervision of Dr. Barbara Herrmann, Dr. John Guinan, and Dr. Steven Rauch. Kim got the opportunity to present her work at multiple national and international conferences. She won travel awards for the $41^{\text {st }}$ and $42^{\text {nd }}$ MidWinter meetings of the Association for research in Otolaryngology in San Diego, California and Baltimore, Maryland. In addition, she received the 2019 Jo Kolk Scholarship facilitated by the Dutch Society for Women with an Academic Education. During her time as a PhD student, Kim coordinated the weekly Work in Progress Seminar for all research laboratories at Mass. Eye and Ear (2018-2019) and was elected Chair of the student, postdoc and medical resident chapter of the Association for Research in Otolaryngology (spARO 2019-2020). 
